\title{
Analysis of Variables which affect the Mechanical and Fracture Properties of Porcine Skin
}

\author{
by
}

Trevor Irwin

A thesis submitted to the Faculty of Graduate and Postdoctoral Affairs in partial fulfillment of the requirements for the degree of

Master of Applied Science

in

Mechanical Engineering

Carleton University

Ottawa, Ontario

(C) 2020, Trevor Irwin 


\begin{abstract}
A diabetes infusion kit is a treatment option for patients with type one diabetes. Using beam springs, the device inserts a Teflon cannula into a patient's skin for delivery of insulin. Modelling the insertion process requires understanding of how the skin surface deforms and fractures. Experiments measuring the indentation properties and fracture properties of porcine skin were performed. A viscoelastic model was developed and fit to experimental indentation force results to derive material properties. A fracture toughness coefficient was derived from the experimental fracture force results. The effect independent variables had on variations in experimental force and derived material properties was investigated. The changes in results due to changes in independent variables mostly conformed to the assumptions made about the behaviour of skin tissue as a viscoelastic material. The results obtained in the experiments performed had good agreement to other results in published literature.
\end{abstract}




\section{Acknowledgements}

I would like to thank Andrew Speirs and Craig Merrett for their guidance and patience. Their suggestions of how to overcome the many issues that arose during the design of the experiments was invaluable.

I would also like to thank Mary-Jo Weir-Weiss for her contribution in setting up the study problem and her help in the preliminary experimental work.

I would finally like to thank the Mechanical and Aerospace departmental staff, specifically Alex Proctor and David Raude for their help in the fabrication of the apparatus and help in providing equipment and tools. Their contributions made the fabrication and calibration process quick and painless. 


\section{Table of Contents}

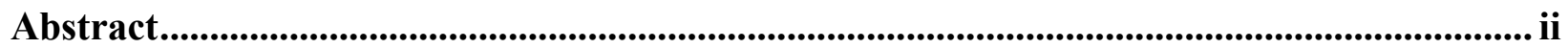

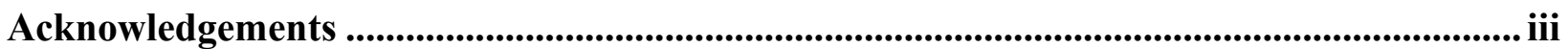

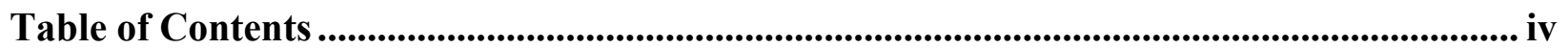

List of Tables ............................................................................................................................. vi

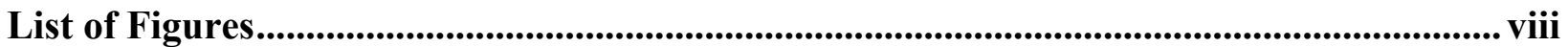

List of Appendices.................................................................................................................................. xii

Nomenclature and Symbols ....................................................................................................................... xiii

Chapter 1: Introduction ................................................................................................. 1

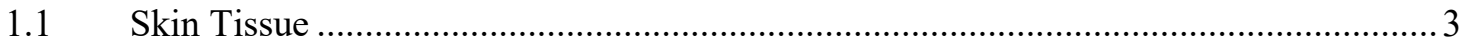

$1.2 \quad$ Collagen in Other Tissue Structures.....................................................................

$1.3 \quad$ Uniaxial Tension Testing of Skin Tissue …………….............................................

$1.4 \quad$ Indentation of Skin Tissue................................................................................. 10

$1.5 \quad$ Previous Work in Skin Indentation ......................................................................11

1.6 Mathematical Model for the Indentation of Skin .........................................................15

$1.7 \quad$ Estimation of Strain and Tip/Skin Contact Area ........................................................ 16

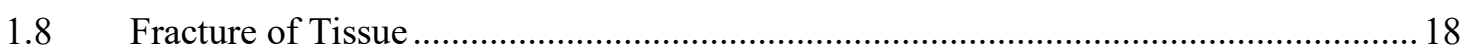

1.9 Previous Work in Tissue Fracture ...........................................................................21

$1.10 \quad$ Mathematical Model for the Fracture of Tissue .............................................................23

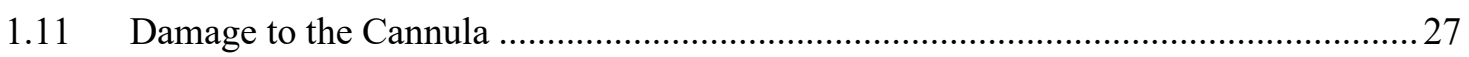

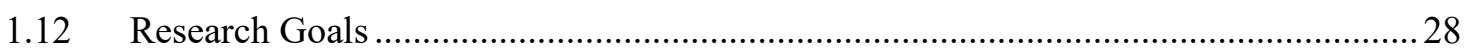

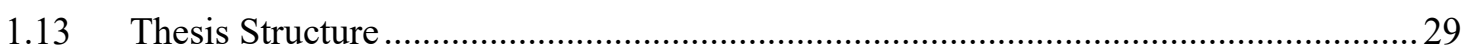

Chapter 2: The Effect of Surface Tension, Indentation Speed and Tip Contact Area on the Shear Modulus and Viscoelastic Relaxation Periods of Porcine Tissue .................................. 30

$2.1 \quad$ Differences from the Journal Submission Version ........................................................ 30

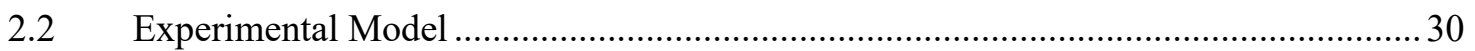

2.2.1 Estimation of Strain and Tip/Skin Contact Area....................................................... 31

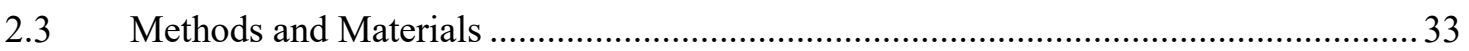




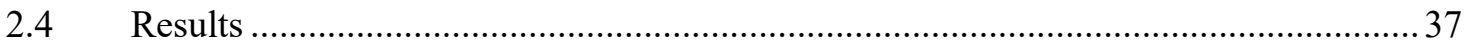

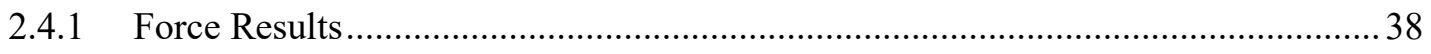

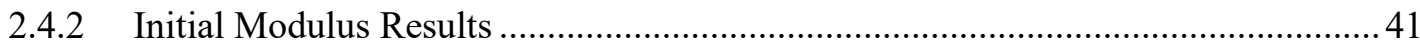

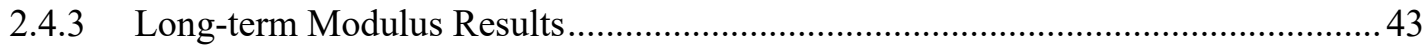

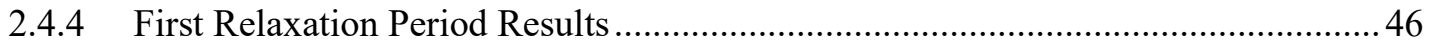

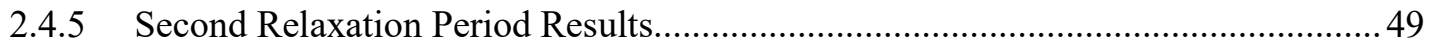

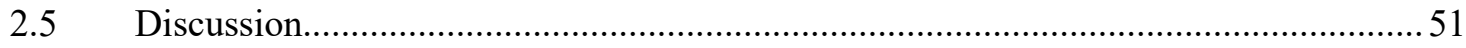

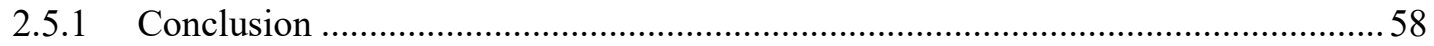

\section{Chapter 3: Effect of Surface Tension, Needle Diameter and Insertion Velocity on the}

Fracture Properties of Porcine Tissue ...............................................................................6 60

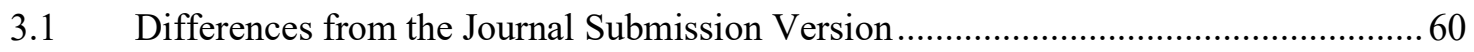

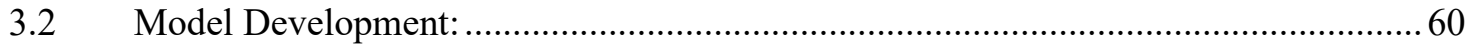

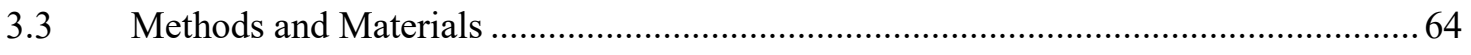

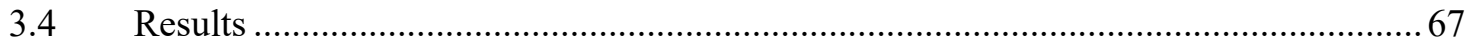

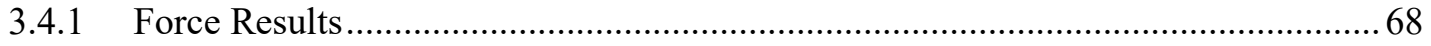

3.4.2 Fracture Toughness Coefficient Results ........................................................... 70

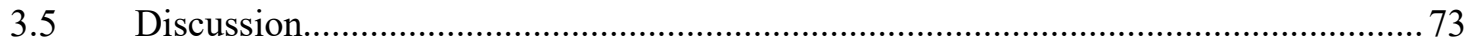

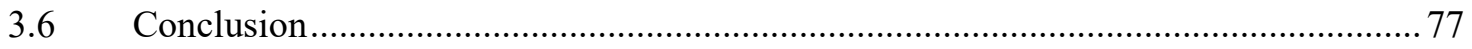

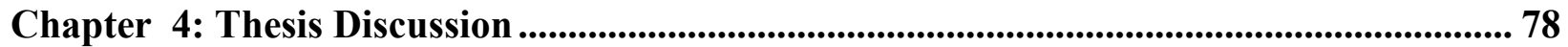

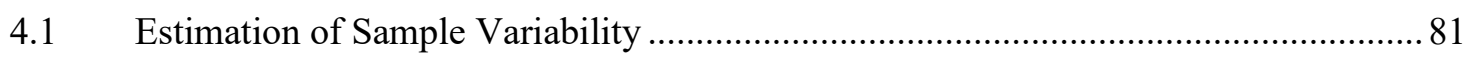

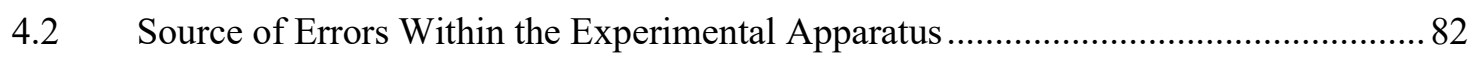

4.3 Comparing the Experimental Apparatus to the Manufactured Infusion Kit.................85

Chapter 5: Thesis Conclusion ............................................................................................ 87

Chapter 6: Contributions to the Field............................................................................................. 88

Chapter 7: Future Work ............................................................................................................. 89

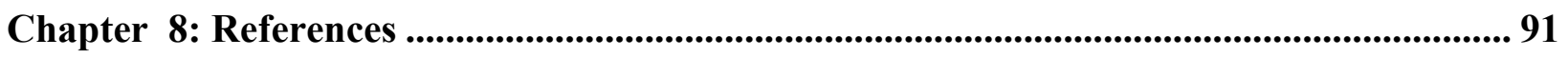




\section{List of Tables}

Table 1: Seven commonly used hyperelastic models [21]-[26] ......................................................

Table 2: Indenter tip contact surface area and induced strain results assuming an indentation of $2.5 \mathrm{~mm} 37$

Table 3: Step-wise regression results of the maximum force. Indentation speed and indenter tip surface area were significant independent variables $(\mathrm{p}<0.05)$ applied surface area was not significant and was omitted from the final regression equation. The beta value of speed is an order of magnitude larger than surface area indicating speed has a greater effect on the maximum force. The $\mathrm{R}^{2}$ value of 0.094 indicates that speed and surface area have a weak relationship to the maximum force. .39

Table 4: Step-wise regression results of the initial modulus. Applied tension, indentation speed and indenter tip surface area were significant independent variables $(p<0.05)$. The beta values of surface area and speed are an order of magnitude larger than tension and the beta value of surface area is larger than that of speed. This indicates surface area has the greatest effect on the initial modulus followed by speed then tension. The $\mathrm{R}^{2}$ value of 0.65 indicates that the independent variables explain the variance of the initial modulus well

Table 5: Step-wise regression results of the long-term modulus. Applied tension, indentation speed and indenter tip surface area were significant independent variables $(\mathrm{p}<0.05)$. The beta values of surface area and speed are an order of magnitude larger than tension and the beta value of surface area is larger than that of speed. This indicates surface area has the greatest effect on the long-term modulus followed by speed then tension. The $\mathrm{R}^{2}$ value of 0.64 indicates that the independent variables explain the variance of the long-term modulus well.

Table 6: Step-wise regression results of the first relaxation period. Applied skin tension, indentation speed and indenter tip surface area were significant independent variables $(\mathrm{p}<0.05)$. The beta value of tension and speed are an order of magnitude larger than surface area and approximately equal. This indicates that tension and speed have an equal effect on the first relaxation period and surface area has a lower effect. The $\mathrm{R}^{2}$ value of 0.073 indicates that tension, speed and surface area have a weak relationship to the first 
relaxation period.

Table 7: Step-wise regression results of the second relaxation period. Indentation speed and indenter tip surface area were significant independent variables $(\mathrm{p}<0.05)$ applied surface area was not significant and was omitted from the final regression equation. The beta value of speed and surface area are approximately equal indicating an equal effect on the second relaxation period. The $\mathrm{R}^{2}$ value of 0.073 indicates that speed and surface area have a weak relationship to the second relaxation period. .49

Table 8: Step-wise regression results of the maximum force. Insertion speed approached significance as an explanatory variable but was omitted from the final regression equation. Applied skin tension and needle diameter were significant independent variables $(\mathrm{p}<0.05)$. The similar beta values indicate that both tension and diameter have an approximately equal effect on the maximum force. The $\mathrm{R}^{2}$ value of 0.12 indicates that tension and diameter have a weak relationship to maximum force.

Table 9: Step-wise regression results of the fracture toughness. Insertion speed and needle diameter were not significance as an explanatory variable $(\mathrm{p}<0.05)$ and were omitted from the final regression equation. Applied skin tension was a significant independent variable $(\mathrm{p}<0.05)$. The $\mathrm{R}^{2}$ value of 0.12 indicates that tension has a weak relationship to fracture toughness. 71 


\section{List of Figures}

Figure 1: Medtronic MiniMed Mio infusion device ..................................................................... 1

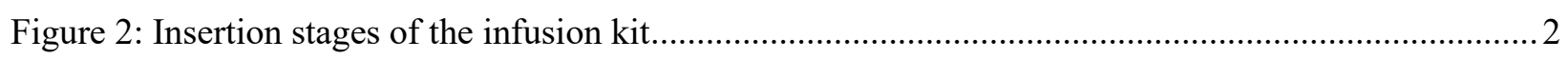

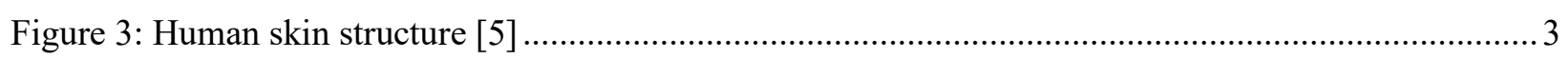

Figure 4: Mechanical response of collagen to loading. Part A shows a representation of the individual collagen fibers deforming under a load F. Part B shows the contributions of each fiber as it is activated due to the deformation caused by load F. Part C shows the force strain curve that would be produced by

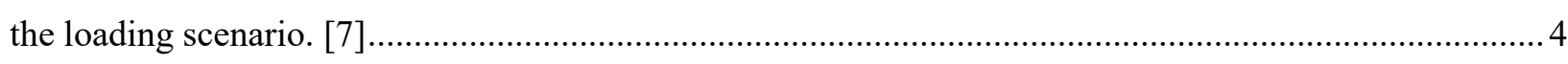

Figure 5: Typical stress strain curve of human tissue [9] ............................................................. 4

Figure 6: Kelvin-Voigt model [43]. Where the g's are the spring constants and $\boldsymbol{\mu}$ is the damping

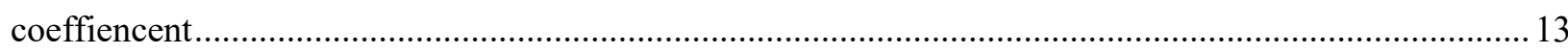

Figure 7: Generalized Maxwell model [45]. Where the M's are the spring constant and $\boldsymbol{\eta}$ is the damping

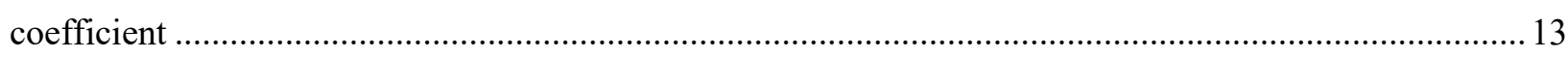

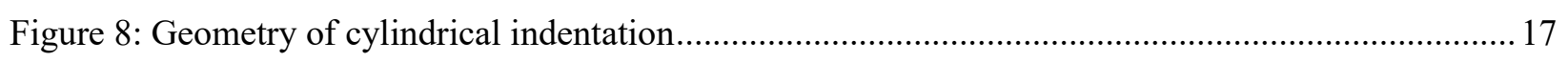

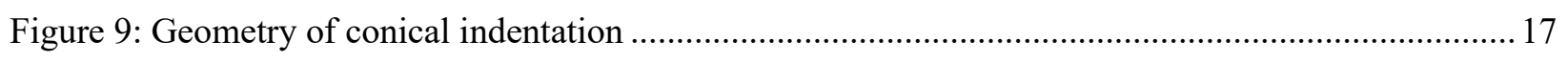

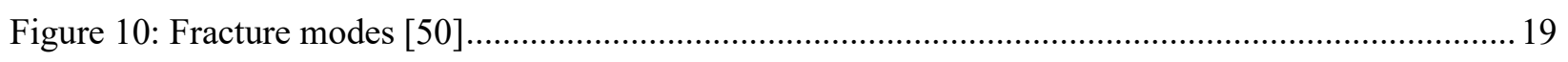

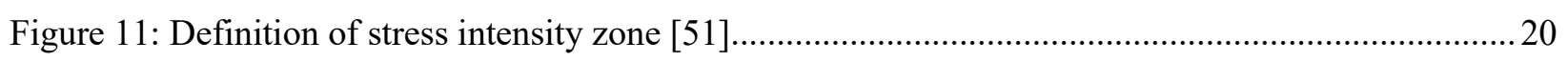

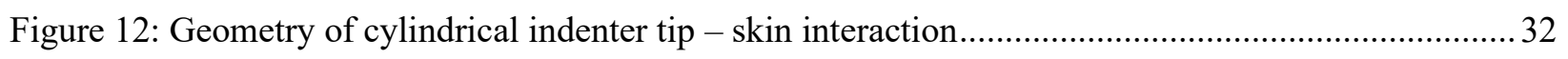

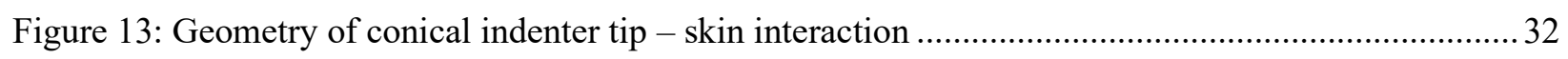

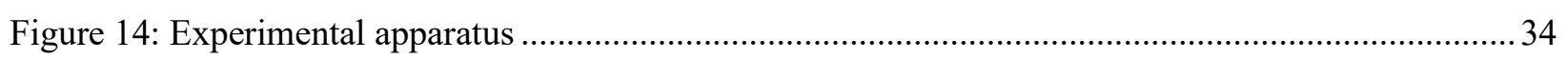

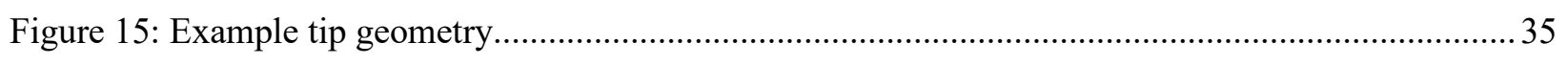

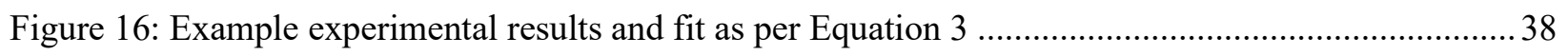

Figure 17: Measured maximum force plotted against applied tension; the red line shows the pair-wise relationship from a simple regression. Changes in tension were not found to be significant $(p=0.18)$ in 
affecting the maximum force.

Figure 18: Measured maximum force plotted against indentation speed; the red line shows the pair-wise relationship from a simple regression. Changes in speed were significant $(\mathrm{p}<0.05)$ in affecting the maximum force. .40

Figure 19: Measured maximum force plotted against indenter tip surface area; the red line shows the pairwise relationship from a simple regression. Changes in surface area were significant $(p<0.05)$ in affecting the maximum force. .40

Figure 20: Calculated initial modulus plotted against applied tension; the red line shows the pair-wise relationship from a simple regression. Changes in tension were significant $(\mathrm{p}<0.05)$ in affecting the initial modulus

Figure 21: Calculated initial modulus plotted against indentation speed; the red line shows the pair-wise relationship from a simple regression. Changes in speed were significant $(\mathrm{p}<0.05)$ in affecting the initial modulus .42

Figure 22: Calculated initial modulus plotted against indenter tip surface area; the red line shows the pairwise relationship from a simple regression. Changes in surface area were significant $(p<0.05)$ in affecting the initial modulus. .43

Figure 23: Calculated long-term modulus plotted against applied tension; the red line shows the pair-wise relationship from a simple regression. Changes in tension were significant $(\mathrm{p}<0.05)$ in affecting the longterm modulus.

Figure 24: Calculated long-term modulus plotted against indentation speed; the red line shows the pairwise relationship from a simple regression. Changes in speed were significant $(p<0.05)$ in affecting the long-term modulus.

Figure 25: Calculated long-term modulus plotted against indenter tip surface area; the red line shows the pair-wise relationship from a simple regression. Changes in surface area were significant $(\mathrm{p}<0.05)$ in affecting the long-term modulus. .46

Figure 26: Calculated first relaxation period plotted against applied tension; the red line shows the pair- 
wise relationship from a simple regression. Changes in tension were significant $(p<0.05)$ in affecting the first relaxation period.

Figure 27: Calculated first relaxation period plotted against indentation speed; the red line shows the pairwise relationship from a simple regression. Changes in speed were significant $(p<0.05)$ in affecting the first relaxation period. .48

Figure 28: Calculated first relaxation period plotted against indenter tip surface area; the red line shows the pair-wise relationship from a simple regression. Changes in surface area were significant $(\mathrm{p}<0.05)$ in affecting the first relaxation period. .48

Figure 29: Calculated second relaxation period plotted against applied tension; the red line shows the pair-wise relationship from a simple regression. Changes in tension were not found to be significant $(\mathrm{p}=0.16)$ in affecting the second relaxation

Figure 30: Calculated second relaxation period plotted against indentation speed; the red line shows the pair-wise relationship from a simple regression. Changes in speed were significant $(\mathrm{p}<0.05)$ in affecting the second relaxation period. .50

Figure 31: Calculated second relaxation period plotted against indenter tip surface area; the red line shows the pair-wise relationship from a simple regression. Changes in surface area were significant $(\mathrm{p}<0.05)$ in affecting the second relaxation period.

Figure 32: Experimental apparatus

Figure 33: Recorded fracture force trial example

Figure 34: Measured maximum force plotted against applied tension; the red line shows the pair-wise relationship from a simple regression. Changes in tension were found to be significant $(p<0.05)$ in affecting the maximum force.

Figure 35: Measured maximum force plotted against insertion speed; the red line shows the pair-wise relationship from a simple regression. Speed was found to approach statistical significance $(\mathrm{p}=0.056)$ in affecting the force.

Figure 36: Measured maximum force plotted against needle diameter; the red line shows the pair-wise 
relationship from a simple regression. Changes in diameter were found to be significant $(\mathrm{p}<0.05)$ in

affecting the maximum force.

Figure 37: Measured fracture toughness coefficient plotted against applied tension; the red line shows the pair-wise relationship from a simple regression. Changes in tension were found to be significant $(\mathrm{p}<0.05)$

in affecting the fracture coefficient.

Figure 38: Measured fracture toughness coefficient plotted against insertion speed; the red line shows the pair-wise relationship from a simple regression. Changes in insertion speed were not found to be significant $(\mathrm{p}=0.195)$ in affecting the fracture coefficient. 72

Figure 39: Measured fracture toughness coefficient plotted against needle diameter; the red line shows the pair-wise relationship from a simple regression. Changes in diameter were not found to be significant $(\mathrm{p}=$ $0.195)$ in affecting the fracture coefficient.. .72

Figure 40: Tip geometry of hypodermic needle. .74

Figure 41: Tip geometry of the tattoo needle .74 


\section{List of Appendices}

Appendix A: Supplementary Figures.

Appendix B: Equipment List and Drawings. ................................... 108

Appendix C: Calibrations........................................................ 116

Appendix D: Matlab and Arduino Codes. ....................................... 132

Appendix E: Experimental Data Example....................................... 151

Appendix F: Instrumentation Data Sheets. ..................................... 157 


\section{Nomenclature and Symbols}

Symbols and nomenclature are given in the order in which they appear in the thesis

- $\quad W-$ Hyperelastic strain energy density $\left(\mathrm{N} / \mathrm{m}^{2}\right)$ (Section 1.5 only)

- $\quad C_{i}-$ Hyperelastic material coefficients $\left(\mathrm{N} / \mathrm{m}^{2}\right)$ (Section 1.5 only)

- $\quad I_{1}-$ First invariant of the right Cauchy-Green deformation tensor

- $\quad \lambda_{i}-$ Principal stretches (unitless) (Section 1.5 only)

- $\quad \epsilon-$ Strain (unitless)

- $\quad \mu-$ Shear Modulus (Pa) (Section 1.5 only)

- $\quad \alpha-$ Ogden material coefficient (unitless) (Section 1.5 only)

- $\quad E-$ Elastic modulus $(\mathrm{Pa})$

- $\quad v-$ Poisson's ration (unitless)

- $\quad P-$ Reaction force (N) (Section 1.5 only)

- $\quad R-$ Spherical indenter radius (m) (Section 1.5 only)

- $\quad \delta$-indentation depth (m) (Section 1.5 only)

- $\quad g_{i}-$ Spring constants $(\mathrm{N} \cdot \mathrm{m})$ (Section 1.5 only)

- $\quad \sigma-$ Stress $(\mathrm{Pa})$

- $\quad t-$ Time $(\mathrm{s})$

- $\tau-$ Viscoelastic integration variable (s)

- $\quad F-$ Force $(\mathrm{N})$

- $\quad A-$ Surface area $\left(\mathrm{m}^{2}\right)$

- $\quad R-$ Stress and strain affected zone radius (m)

- $\quad u_{z}-$ Indented surface position (m) 
- $\quad D-$ Indentation depth (m)

- $\quad a-$ Separation point radius (m) (Section 1.7, 3.2 only)

- $\quad p$ - Radial distance (m)

- $\quad \alpha-$ Conical tip outside angle $\left({ }^{\circ}\right)$

- $\quad h$ - Separation point height (m)

- $\quad L-$ Length of deformed surface (m)

- $\quad K-$ Stress concentration $(\mathrm{Pa} \cdot \sqrt{ } \mathrm{m})$

- $\quad C-$ Fracture geometric constant (unitless)

- $\quad a-$ Crack length (m) (Section 1.8 only)

- $\quad r$-Radial distance (m)

- $\quad \theta-$ Angle $\left({ }^{\circ}\right)$

- $\quad W-$ Work $(J)$

- $\quad J_{I C}-$ Critical fracture toughness $\left(\mathrm{J} / \mathrm{m}^{2}\right)$

- $\quad U-$ Strain energy work $(J)$

- $\quad \lambda-$ Lamé's first material parameter $(\mathrm{Pa})$

- $\quad \mu$-Lamé's second material parameter $(\mathrm{Pa})$

- $\quad V-$ Volume $\left(\mathrm{m}^{3}\right)$ 


\section{Chapter 1: Introduction}

Diabetes is a disease which inhibits a person's ability to use or produce insulin; insulin is a hormone used to regulate blood sugar. Statistics Canada estimates 2.2 million Canadians are currently diagnosed with diabetes, with approximately twelve thousand of them being youths [1]. Diabetes Canada estimates $10 \%$ of diabetes cases are type 1 ; the complete inability to produce insulin of any kind [2]. Treatment for type 1 diabetes is daily injections of insulin or use of an insulin pump. An insulin pump requires a feed line to deliver insulin subcutaneously; one can be surgically implanted, or one can be inserted every few days. For those who choose to insert a new line every few days there are many ready-made kits available to choose from; one kit is the Medtronic Mini-Mio device, an example given in Figure 1.

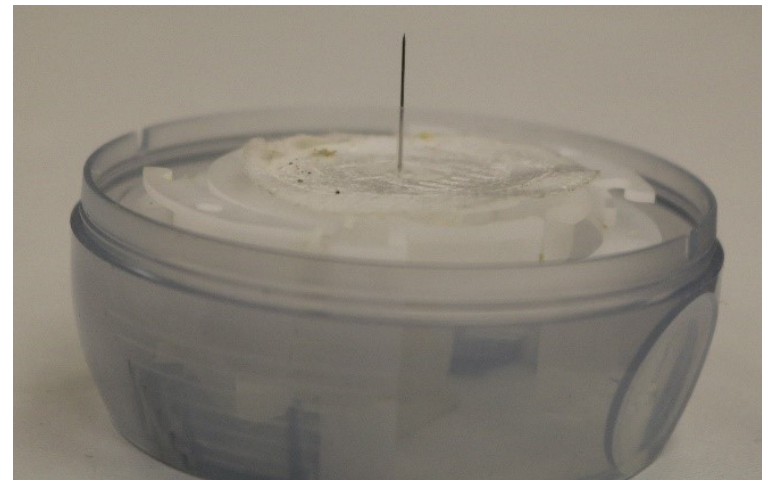

Figure 1: Medtronic MiniMed Mio infusion device
The Mini-Mio infusion kit is a spring driven system that drives a Teflon cannula into the patient's skin using a central needle to break the skin surface. The Teflon cannula is a tube which allows the delivery of liquid insulin directly to the blood stream. Four plastic bars connect the core to the wall of the device. As the core is retracted, the bars bend creating the spring energy for use in inserting the cannula. The needle is most commonly perpendicular to the skin, as Figure 1 illustrates, but can be oblique. The cannula is deposited six to nine millimeters below the skin and the needle is then withdrawn allowing the cannula to be hooked up to the pump unit.

Should the cannula become damaged or constricted the insulin supply will be interrupted; this can be very dangerous for youths with diabetes. Studies of the failure rate of infusion kits 
estimate that $15 \%$ of devices will fail during the insertion process [3], [4]. A review of related literature made clear that there is a significant gap in the engineering understanding of the mechanical response of soft biological tissue to a perpendicular load at non quasi-static conditions and the fracture properties of soft tissues by means of a sharp needle.

There are three stages in the insertion process of the infusion kit as shown in Figure 2. As the spring of the infusion kit is released, the central needle strikes the skin surface causing some deformation perpendicular to the surface of the skin. At some critical point the surface of the skin is fractured by the needle, the surface of the skin will then rebound before coming into contact with the cannula. The cannula is then required to widen the puncture created by the needle, this widening is not instantaneous so the skin will also deform and push back against the cannula. Once the cannula widens the crack sufficiently it can continue through the skin and the skin surface will rebound towards its original resting position. The major events of this scenario are the deformation and eventual release of the skin surface, the fracture of the skin and the widening of the crack created by the needle. The purpose of this thesis is to examine the mechanical response to a deformation load and to a penetrative load of skin tissue.

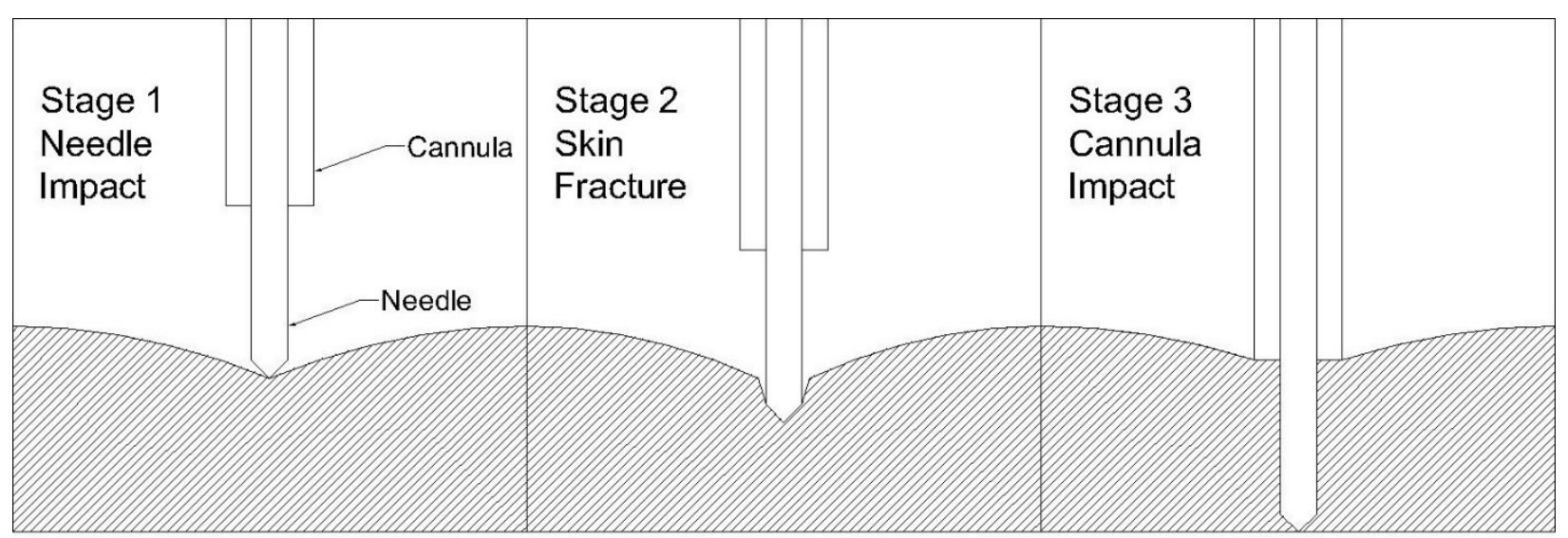

Figure 2: Insertion stages of the infusion kit 


\subsection{Skin Tissue}

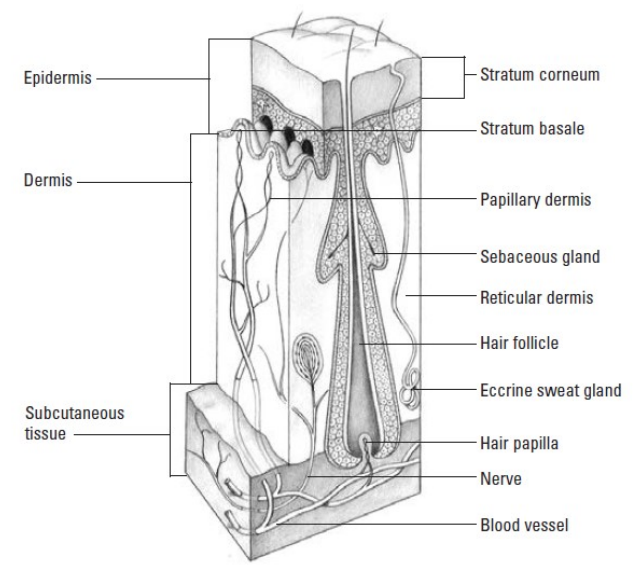

Figure 3: Human skin structure [5]
Skin is composed of three primary layers: the epidermis, dermis and subcutaneous tissue; Figure 3 shows an illustration of the major components of human skin [5], [6]. The epidermis varies in thickness from 0.1 millimetres on the eyelids to a millimeter on the soles of the feet. The dermis is the primary structural layer, and is composed of collagen and

elastin [5]. The collagen and elastin are woven together into a matrix with skin cells and provide tensile strength to the skin layer. The subcutaneous tissue, also called the hypodermis, is a primarily fatty layer where nerve and blood vessels are located. Skin tissue composition and layer thicknesses varies with location on the body, and factors like age and health.

\subsection{Collagen in Other Tissue Structures}

Collagen is primarily a tensile support structure; it is made up of many bundles of collagen fibrils [7]. Collagen fibers arrange themselves into large, ordered bundles in ligaments or tendons; in cartilage or skin, the strands are shorter and more randomly arranged throughout the tissue. When collagen bundles are loaded, they uncrimp until fully extended before taking the full loading. On a stress strain curve there are two distinct loading regions; the first region, often called the "toe" region, is characterized by a large deformation with little load applied followed by a more linear region of increasing stiffness with deformation before breaking [7]-[10]. Individual collagen fibers have been shown to exhibit viscoelastic properties [11]. Figure 4 shows a mechanical approximation of this behaviour and Figure 5 shows a typical stress strain 
curve.
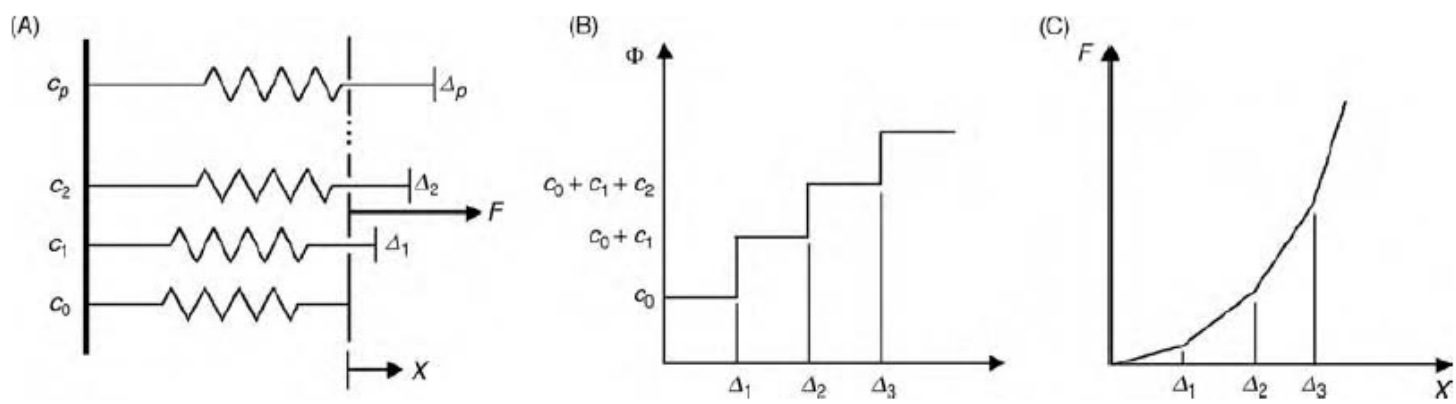

Figure 4: Mechanical response of collagen to loading. Part A shows a representation of the individual collagen fibers deforming under a load F. Part B shows the contributions of each fiber as it is activated due to the deformation caused by load F. Part $\mathrm{C}$ shows the force strain curve that would be produced by the loading scenario. [7]

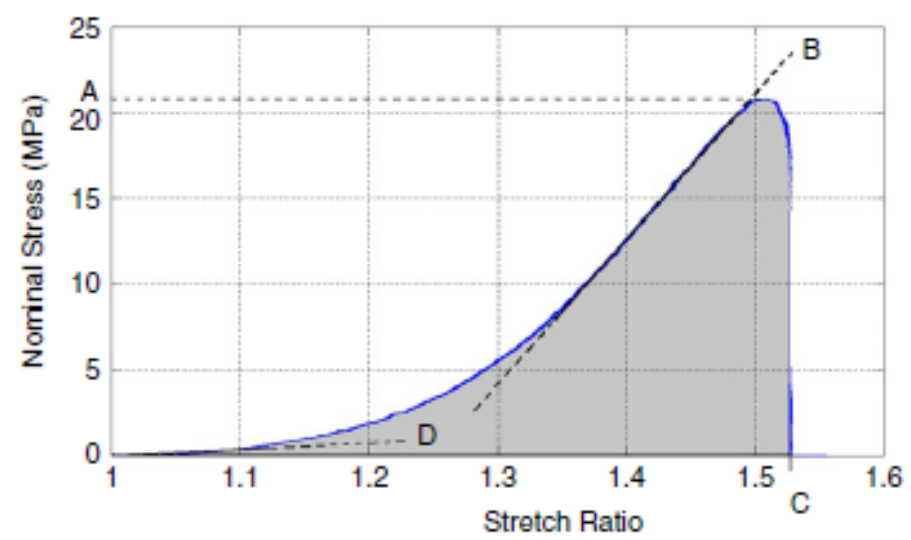

Figure 5: Typical stress strain curve of human tissue [9]
Collagen strands in cartilage are more randomly oriented and the tissue has large quantities of fluid inside, approximately $60 \%$ [7]. When strain is applied fluid flow within the tissue is resisted by the component fibrils throughout the material; the amount of resistance is dependant on the pressure gradients [8]. Hayes et al. and later Julkunen et al. developed a method of estimating the Young's Modulus of articular cartilage tissue subjected to an indentation force [12], [13]. Hayes et al. assumed that the cartilage tissue was elastic to derive an analytical solution. This solution assumed a semi-infinite cartilage layer with finite and defined thickness over a rigid base to simulate the bone. It is known that degenerative diseases change the material properties of cartilage, so this analysis was developed to quantify the changes between healthy and damaged cartilage. Julkunen et al. modified the 
Hayes solution to account for the poroelasticity and biphasic nature of cartilage. The mechanical behaviour of cartilage and skin are different, and the material models are not directly transferable; but the models are a useful beginning point in developing the material model used in this thesis.

Elastin is another important structural component in skin tissue. It is highly elastic and gives skin its recoil ability and resilience [6]. Collagen is approximately a hundred times stronger than elastin [14]. The work done in this thesis does not differentiate between the contributions of elastin and collagen, although collagen is assumed to be the dominant contributor.

\subsection{Uniaxial Tension Testing of Skin Tissue}

There are many types of materials that exhibit hyperelastic properties and many models that could be used to predict the tension properties of tissues. Materials used to test properties of tissues were outlined in a review done by Dąbrowska et al. [15]. Human skin has been used in many of the experiments described in this section, while other investigators used other animal tissue or simulated tissues. No literature has been found that directly compares the mechanical properties of one species' tissue to another.

Skin tissue is known to be anisotropic, hyperelastic and viscoelastic; in human tissue there are preferred lines of naturally occurring tension called Langer lines which also affect the material properties. Ni Annaidh et al. and Ottenio et al. did tension testing of samples taken from the backs of human donors [9], [10]. In quasi-static testing Ni Annaidh et al. found that loading in parallel or at a 45 degree angle to the Langer lines gave significantly better failure properties than when loading perpendicular to the Langer lines. When Ottenio et al. loaded samples at higher strain rates the Langer lines were even more important in influencing the failure 
properties. At quasi-static speed the failure properties were similar for loading in parallel or 45 degree; however, at higher strain rates the failure properties of loading in parallel were significantly better than loading at 45 degree. Failure occurred at stretch ratios of approximately $150 \%$ with both quasi-static and dynamic loading. There is a large variance in these results with differences between samples of up to $80 \%$. Cyclic loading and unloading of breast tissue was performed by Kumaraswamy et al.; this testing was to determine the effect of radiation treatment and scarring on the material properties [16]. It was found that scarred or irradiated tissue lost much of the first phase of the stress strain curve and began to stiffen much more rapidly than the undamaged tissue. Silver et al. performed viscoelastic testing on human tissue and synthetic processed dermis materials to determine the viscous contribution to the tensile force [17]. This data was then used to calculate the fibril length of the collagen fibers within the test materials. The material parameters derived from experiments have been incorporated into finite element models [18], [19]. Given the computational requirements of these models and the natural imperfections of real tissue; it is unclear whether computational models can accurately predict new properties or the engineering performance of tissue materials. A good understanding of skin properties would allow for simulations of many different scenarios, such as, the mechanics of single beard hair cutting as done by Fang and Köppl [20].

Testing of porcine tissue has been done for many of the same scenarios; however, with the greater availability of samples it has allowed comparative studies of hyperelastic models. Two separate experiments compared seven hyperelastic material models: Neo-Hookean, Mooney-Rivlin, Ogden, Yeoh, Humphrey, Martins and Veronda-Westmann models [21], [22]. Table 1 shows a brief description of each model[21]-[26]. 
Table 1: Seven commonly used hyperelastic models [21]-[26]

\begin{tabular}{|l|l|}
\hline Model & Description \\
\hline Neo-Hookean & $\begin{array}{l}\text { Considered a specialization of the Mooney-Rivlin model. Developed for } \\
\text { vulcanized rubber using a three-dimensional network of long-chain molecules. } \\
\text { The derivation originates from incompressible Cauchy-elastic materials. Intended } \\
\text { for isotropic, rubber-like materials and captures nonlinear effects at small strains. } \\
\text { Does not capture the finite extension of molecular chains observed in practice. }\end{array}$ \\
\hline Mooney-Rivlin & $\begin{array}{l}\text { A specialization of the Ogden model. Derived from incompressible Cauchy-elastic } \\
\text { materials for isotropic rubber-like materials. Does not capture finite extension of } \\
\text { molecular chains observed in practice. }\end{array}$ \\
\hline Ogden & $\begin{array}{l}\text { Developed for rubber-like materials using compressible Cauchy-elastic material } \\
\text { formulations. Captures finite strain behaviour. }\end{array}$ \\
\hline Yeoh & $\begin{array}{l}\text { Developed for carbon-black filled rubbers. These materials exhibit substantially } \\
\text { different behaviour than neat rubber, in particular the shear modulus varies. Model } \\
\text { captures stiffening effect at large strains. }\end{array}$ \\
\hline Humphrey & $\begin{array}{l}\text { Developed for tissue using an incompressible, isotropic, nonlinear-elastic } \\
\text { approach. The formulation captures finite strain and twist behaviour through } \\
\text { exponential expressions. }\end{array}$ \\
\hline Martins & $\begin{array}{l}\text { Specialized for soft tissues and incorporates anisotropic Cauchy-elastic behaviour } \\
\text { of the material. Modified from the Humphrey model with an explicit dependence } \\
\text { on the stretch of the fibers. }\end{array}$ \\
\hline $\begin{array}{l}\text { Vharacterizes a soft tissue material that is nonlinear, rate-independent, and } \\
\text { isotropic. The model captures finite extensions. }\end{array}$ \\
\hline
\end{tabular}

The experiments performed by Martins et al. were performed on porcine muscle tissue, while the experiments performed by Łagan and Liber-Kneć were performed on skin tissue from both the abdomen and back tissue. The Martins et al. experiments had much higher correlation coefficients than the Lagan experiments; this is possibly due to the difference in tissues used. Muscle tissue has more ordered collagen than skin tissue which likely accounts for the improved model prediction. In both experiments the Neo-Hookean model had the worst correlation; this model is a strictly linear model expressing the strain energy as shown in Equation 1.

$$
W=C_{1}\left(I_{1}-3\right)
$$

Hyperelastic materials are generally non-linear; skin tissue is especially non-linear. The Yeoh and Martins models provide the best fits in both experiments. The Yoeh model is a cubic 
model as shown in Equation 2 and the Martins model is a double exponential model as shown in Equation 3.

$$
\begin{gathered}
W=C_{1}\left(I_{1}-3\right)+C_{2}\left(I_{1}-3\right)^{2}+C_{3}\left(I_{1}-3\right)^{3} \\
W=C_{1}\left(e^{C_{2}\left(I_{1}-3\right)}-1\right)+C_{3}\left(e^{C_{4}(\lambda-3)}-1\right)
\end{gathered}
$$

These equations are functions of material constants given as $C_{i}$, the first invariant of the right Cauchy-Green deformation tensor given as $I_{1}$ and of the principal stretches given as $\lambda_{i}$ their definition shown in Equation 4.

$$
I_{1}=\lambda_{1}^{2}+\lambda_{2}^{2}+\lambda_{3}^{2} \text { where } \lambda=\epsilon+1
$$

At quasi-static strain rates hyperelastic models provide a good fit for the behaviour of tissue. Since tissue responds viscoelastically at non quasi-static speed, the response is strain rate and cyclically dependant. Experiments on the cyclic properties of porcine tissue have been done to determine the effect of preconditioning [27]. Preconditioning is a known condition in other hyperelastic materials like rubbers; as more strain cycles are applied to a sample the peak force response decreases. The results of the experiments performed by Remache et al. showed that there was a drop in the peak response as more cycles were applied to the porcine tissue samples [27]. The results also show that there was a larger response for an applied strain rate of $1 \mathrm{~mm} / \mathrm{s}$ than a rate of $0.1 \mathrm{~mm} / \mathrm{s}$. Preconditioning is an important property for tensile testing, however, since the experiments performed for this thesis were not cyclic it was not considered for the analysis. In a real patient, the skin would not be preconditioned but under a normal state of natural tension. The experiments were designed to mimic this as best as possible.

The premise of anisotropy in the skin and strain dependant response has also been shown in experiments on the tissue of mice and rats. Karimi et al. tested mouse abdominal and back skin in two directions; the results show a significant difference between the different testing 
directions [28]. Interestingly the results show that the back and abdominal tissue reacts differently; the largest peak stress occurred in the abdominal tissue tested in the axial direction and on the back tissue in the circumferential direction. From this it can inferred that the anatomical location of the tissue sample greatly affects both the properties and the preferential loading direction. Nicolle et al. tested rat tissue under shearing conditions to determine its response [29]. They found that there was a slight region of strain softening before the typical strain hardening regime seen in the uniaxial testing. This result could indicate that there are regions of strain rates which change the properties in atypical fashions.

In vivo extensometer testing is a good way of correlating the tension testing. In these tests, external tips press perpendicularly into the skin surface, apply a deformation to the skin and the force response is measured. Facial tissue extension was performed by Then et al.; they performed a ramp and hold test to investigate the unconditioned response and the relaxation rate of in vivo tissue [30]. Their results matched well with the tension testing performed on other tissues. Jacquet et al. performed a simpler extension test on forearms, legs and abdomens of volunteers; their results showed a clear difference in response of different anatomical locations [31]. They also were able to obtain results showing that orientation of extension at the same site also affected the response. Dynamic testing has also been performed using a method similar to an extensometer. Sandford et al. compared the properties of skin at different forearm locations and the effect hydration and topical formulations had on those properties [32]. Their analysis derived a stiffness and damping coefficient of the skin; the results showed differences in these properties with both skin hydration and application of topical formulations. 


\subsection{Indentation of Skin Tissue}

The experiments described above investigated the in-plane loading of skin tissue; however, in vivo it is nearly impossible to truly load skin surface in that fashion. Most devices and real-world scenarios apply a force perpendicular to the skin surface. This configuration presents two key challenges: skin composition is not homogenous throughout its thickness and depending on the anatomical location in question skin might cover fat, muscle or bone, which all have vastly different mechanical properties. In the context of the insulin delivery system the problem is a little more defined. The cannula is a maximum of six to nine millimetres in length and is inserted into the abdomen, a region with higher fat content. In fact, in patients with lean body types it is recommended the insertion site be moved to the buttocks to avoid attempting to insert the cannula through any muscle layer.

The indentation properties of skin have uses beyond pure modelling; changes in the mechanical properties of skin can be used as a diagnostic tool for detection of diseases and cancers, and to assess the effectiveness of wound healing or plastic surgery. The majority of indentation testing has been done in vivo on human volunteers due to the non-invasiveness of the indentation procedure. This method of testing has one key deficiency, it cannot control for the effect of skin tension on the mechanical properties. Nor has there been extensive variation in indenter tip geometry or indentation speed. The effects of tip geometry, skin tension and indentation speed on the mechanical properties of skin tissue are three of the experimental subjects contained within this thesis. 


\subsection{Previous Work in Skin Indentation}

Devices for testing skin indentation properties have already been created; these devices are used to assess skin stiffness for use in dermatology. These devices are used to measure changes in skin stiffness to diagnose changes in skin health. Some experiments have been performed to assess the accuracy of these devices. These experiments used devices created by Delfin Technologies to measure the reaction to indentations [33], [34].

Other work in skin indentation has generally taken two approaches: fitting to a hyperelastic model or determining a Young's Modulus. Fitting a hyperelastic model to a perpendicular indentation force is an interesting choice. Skin is demonstrably hyperelastic inplane; applying a load will cause strain perpendicularly, however, due to the toe region as described in section 1.2 there is significant deformation before much contribution of the collagen fibers to resisting the deformation. In cases of indentation the amount of deformation is constrained by anatomy; the thickness of skin is quite small and below the skin layer are muscle and bone which do not react hyperelastically. Muscle and bone have significantly larger Young's Moduli and will rapidly overshadow the contribution of the skin to the reaction force. Tran et al. used an indenter and MRI to create a finite element model; their experiment indented a forearm a maximum of nine millimetres from the surface and used the MRI to take images of the deformation [35]. They used a Neo-Hookean model to describe the response of the skin; the experiments by Łagan and Martins showed that the Neo-Hookean model had the worst fit of the seven hyperelastic models [21], [22]. Despite this, the simulation prediction and experimental results presented by Tran et al. had good agreement. Groves et al. used an Ogden model for their investigation into microneedle design [36]. They used an indentation depth of only $0.3 \mathrm{~mm}$ to extract the Odgen material parameters and create a finite element model that predicts the 
experimental results. The Ogden material model is a commonly used model and fits extremely well to the experimental data of Łagan and Martins [21], [22]. The Ogden model is given in Equation 5 where $\mu$ is the shear modulus, $\alpha$ is a material constant and $\lambda$ are the principal stretches described in Equation 4.

$$
W=\frac{2 \mu}{\alpha^{2}}\left(\lambda_{1}^{\alpha}+\lambda_{2}^{\alpha}+\lambda_{1}^{-\alpha}+\lambda_{2}^{-\alpha}-3\right)
$$

Deriving a Young's Modulus from an indentation load draws on Sneddon's solution of the Boussinesq problem [37]. The Boussinesq problem assumed a rigid indenter pushing on an elastic half-space; this is the first limitation of this derivation. At quasi-static speeds the assumption of a linearly elastic stress-strain regime for tissue is reasonable. Despite knowing that tissue is hyperelastic there are the two distinct regions of stress-strain; both could be approximated by linear functions. As long as the strains applied to the tissue fall wholly within the first "toe" region, its behaviour could be approximated by a linear elastic fit. In the case of an indenter tip deforming the surface of skin, the effective Young's Modulus is given in Equation 6, where subscripts 1 and 2 refer to the skin and indenter respectively. If the indenter is made of metal its Young's modulus is orders of magnitude higher than that of skin and the second term can be neglected.

$$
\frac{1}{E^{*}}=\frac{1-v_{1}^{2}}{E_{1}}+\frac{1-v_{2}^{2}}{E_{2}}
$$

Experiments using this method have been done with varying goals. Pailler-Mattei et al. used this indentation method to derive Young's Moduli for the dermis, hypodermis and underlying muscle layer [38]. They used three springs in series to model their results using assumed thicknesses of each layer. Isaza and Ramirez investigated the effect of temperature and indenter diameter on this elastic Young's Modulus [39]. Álvarez-Asencio et al. used a nano- 


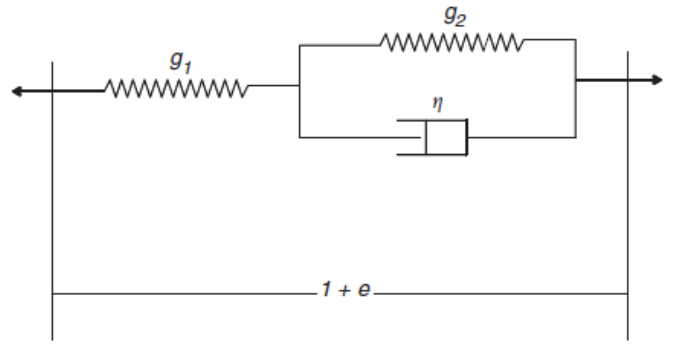

Figure 6: Kelvin-Voigt model [43]. Where the g's are the spring constants and $\mu$ is the damping coeffiencent

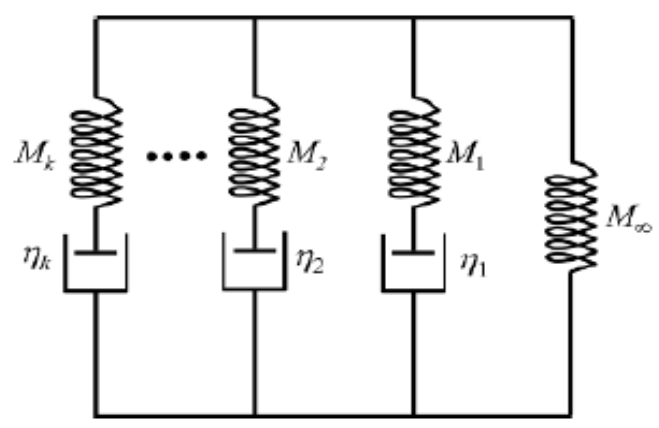

Figure 7: Generalized Maxwell model [45].

Where the $M$ 's are the spring constant and $\eta$

is the damping coefficient indenter made from human hair to derive the properties of the stratum corneum (the outermost layer of the skin) [40]. They used the experimental results to derive a finite element model that would predict the deformation from an applied load. In the above experiments there was an assumption of no slip condition between the indenter and the skin surface. Dai et al. and Chen et al. performed experiments to see if frictional properties of skin had any effect on its indentation properties [41], [42]. The experiments were all done at non quasi-static speed but did not factor in any viscoelastic properties into their analysis.

Models which contain viscoelastic elements can be represented as arrangements of springs and dashpots. There are two commonly used models: Kelvin-Voigt and Maxwell. A Kelvin-Voigt model is a spring in series with a spring and dashpot in parallel as shown in Figure 6. A generalized Maxwell model is a spring in parallel with a number of springs and dashpots in series as shown in Figure 7. Jachowicz et al. used a Kelvin-Voigt model in their analysis; the single spring representing the elastic contribution and the spring dashpot representing the shortterm viscous contribution [43]. They performed both a cyclic indentation and a ramp and hold test to extract parameters of stress relaxation and Young's Modulus. It is important to note that their stress relaxation experiments were performed on synthetic skin models, not in vivo like 
their cyclic indentation test. They plotted the loading and unloading curve of the cyclic indentations; the difference in area of the hysteresis loop was interpreted to be the energy loss due to viscous effects. From this area difference they derived a hysteresis loss factor which is an empirical material coefficient. The spring constants of the two springs (termed $\mathrm{g}_{1}$ and $\mathrm{g}_{2}$ ) were derived from the stress relaxation experiments. The equations for the initial reaction force and the long-term reaction force are given in Equations 7 and 8; where $R$ is the spherical indenter radius, $P$ is the reaction force and $\delta$ is the indentation depth.

$$
\begin{gathered}
P(t=0)=P_{0}=\frac{8}{3} R^{0.5} \delta_{0}^{1.5} g_{1} \\
P(t \rightarrow \infty)=\frac{8}{3} R^{0.5} \delta_{0}^{1.5} \frac{g_{1} g_{2}}{g_{1}+g_{2}}
\end{gathered}
$$

Both Kelvin-Voigt and Maxwell models are viscoelastic models; Kelvin-Voigt is generally used for solid materials and Maxwell for liquid materials [44]. This presents a dilemma for use in tissues; many tissues are solid yet have a very high fluid content. For this reason, a Maxwell model was chosen as the viscoelastic model used in the analysis of indentation forces. The Maxwell model has been used by other researchers; Liu et al. present a mathematical model for the indentation response of a viscoelastic material with a hard surface skin [45]. Their mathematical derivation is similar to the one which will be presented in the next section. Yazdi et al. performed in vivo indentation experiments on human thigh skin, experiments similar in objective to the ones that were performed for this thesis [46]. Like Liu their mathematical derivation for the response was similar to the one which will be presented. Yazdi applied a ramp force to subject's thighs and held the $0.5 \mathrm{~mm}$ displacement for 10 seconds. They then measured the reaction force and derived the viscoelastic parameters. 


\subsection{Mathematical Model for the Indentation of Skin}

The stress in a viscoelastic material is given by Equation 9 [44], where $\sigma$ is the stress state, $\mathrm{E}$ is the modulus of the material, $\tau$ is the integration variable, $\mathrm{t}$ is the time and $\epsilon$ is the strain.

$$
\sigma_{i j}=\int_{-\infty}^{t} E_{i j k l}(t-\tau) \frac{\delta \epsilon_{k l}(\tau)}{\delta \tau} \delta \tau
$$

If the strain is assumed to be instantaneously applied by a step function, then the strain becomes constant for $\mathrm{t}>0$ and 0 for $\mathrm{t}<0$. A general Maxwell model is used, spring constants and damping coefficients are required, and an assumption of uniaxial stress is applied. The strain can be factored out of the integral in Equation 9; the remainder of the integration is evaluated, resulting in the uniaxial stress state, Equation 10.

$$
\sigma=\epsilon\left(E_{\infty}+E_{1} e^{-\frac{t}{\tau_{1}}}+E_{2} e^{-\frac{t}{\tau_{2}}}\right)
$$

Tissue is essentially a composite; the interstitial fluid and the collagen, each will have relaxation times and contribute in different magnitudes. The analysis will require coefficients for the two parts of the tissue matrix. Thus, Equation 10 uses a two term Prony series instead of one with more terms that may provide a better fit to experimental data.

There has been some discussion whether $E_{1}$ and $E_{2}$ are physically meaningful or if they are simply fitting terms [47]. This model assumes that they are fitting terms and thus does not constrain them to be solely positive (which would be the case if they were physical moduli). There are two meaningful modulus values that are derived from this equation: $E_{0}$ and $E_{\infty}$. $E_{0}$ is the summation of $E_{\infty}, E_{1}$ and $E_{2}$ at $\mathrm{t}=0$ and represents the initial elastic response to the applied strain. $E_{\infty}$ is the long-term elastic modulus of the material which could be viewed as an equivalent Young's Modulus. The two relaxation periods $\tau_{1}$ and $\tau_{2}$ are interpreted to be the short 
and long-term stress relaxation periods of the material. The two relaxation periods are possibly properties of the interstitial fluid and the collagen fibers; however, more investigation is required to confirm this hypothesis. Since force was recorded during experimentation the stress in Equation 10 is decomposed into Equation 11.

$$
F=A_{\text {tip }} \epsilon_{\text {surface }}\left(E_{\infty}+E_{1} e^{-\frac{t}{\tau_{1}}}+E_{2} e^{-\frac{t}{\tau_{2}}}\right)
$$

\subsection{Estimation of Strain and Tip/Skin Contact Area}

The estimation of the amount of strain is key to this analysis, there exists some strain affected zone within the sample. If the strain affected zone is assumed to be an infinite plane, then the strain in Equation 11 becomes zero. The depth and extent of the strain affected zone is not readily apparent. Gao et al. performed experiments using needles and simulated tissue [48]. They found the radius of the indented zone was approximately 1.67 of the needle depth at puncture of the skin. This ratio is taken to be the maximum extent of the strain affected zone from the indenter's outer radius given as $R$. With a fixed, assumed indenter depth of $2.5 \mathrm{~mm}$ in all tests that zone is $R=4.175 \mathrm{~mm}$.

Skin is commonly assumed to be incompressible along all three axes; applying a compressive force will not result in any change in volume. With this assumption, the deformation of the skin is simplified to the change in length of a 2-D beam, with the unstrained state being the radius of the strain affected zone. Neglecting strain through the thickness is valid for an infinite half space; since real scenarios are not infinite the experimental set up ensured that there was at least four times the thickness as the indentation depth. 
In the case of the cylindrical indenters,

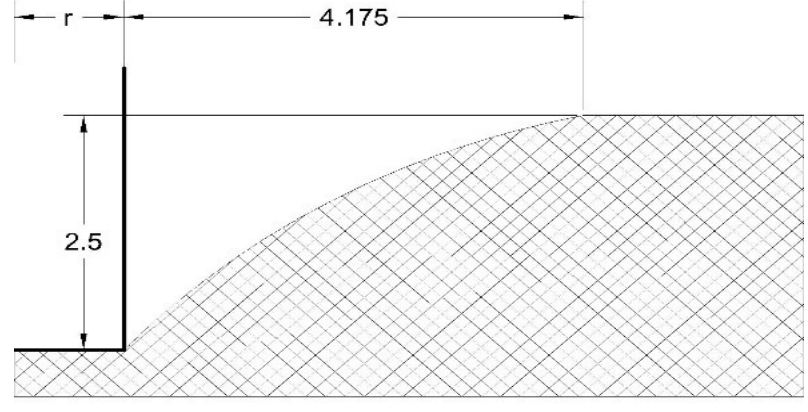

Figure 8: Geometry of cylindrical indentation it will be assumed that the strain affected zone will extend from the edge of the indenter face. The strain affected zone will become the indenter radius plus the assumed zone of 4.175 $\mathrm{mm}$. Figure 8 shows the geometry of the model. Sneddon's solution to indentation in an elastic half space gives an estimate of the deformed surface [37]. Equation 12 is the shape of the surface of an elastic half space when indented by a cylindrical indenter. Where $D$ is the indentation depth, $a$ is the indenter radius and $p$ is the radial distance from the center of the indenter.

$$
u_{z}=\frac{2 D}{\pi} \sin ^{-1}\left(\frac{a}{p}\right), p>a
$$

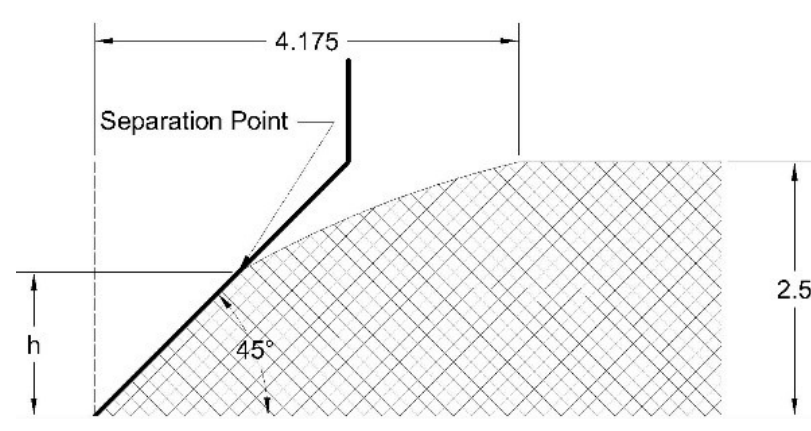

Figure 9: Geometry of conical indentation

The strain estimate of the conical indenters is derived in a similar manner. Since the conical indenters end in a point instead of a flat face, the strain affected zone will equal $R$ in all cases. The tip will have full contact for some height from the tip $h$ before the surface will no longer have contact with the indenter. Figure 9 shows the geometry of the model with the location of the separation point of the conical indenter and the skin surface.

The radius, $a$, and height, $h$, at which the surface detaches from the surface of the cone is given by Equations 13 and 14. Where $\alpha$ is the outside angle of the tip as shown in Figure 9, given 
as $45^{\circ}$. The deformed surface is then given by the Equation 15 .

$$
\begin{gathered}
a=\frac{2 D}{\pi \tan \alpha} \\
h=\frac{\sin (90-\alpha)}{a} \\
u_{z}=\frac{2 D}{\pi a}\left(a \sin ^{-1}\left(\frac{a}{p}\right)-p+\sqrt{\left(p^{2}-a^{2}\right.}\right), p>a
\end{gathered}
$$

The boundary condition of $u=0$ at $p=R+a$ for the cylindrical indenter and at $p=R$ for the conical indenter results in a mathematical discontinuity $\left(u_{z} \neq 0\right)$ at those points; however, without direct measurement of the surface deformation this limitation is unavoidable. The assumption of a linear elastic material is part of the reason this discontinuity exists. The length of these curves is determined by numerical integration with Equation 16. The strain is calculated from the difference between the undeformed surface and the length of the deformed surface.

$$
L=\int_{a}^{b} \sqrt{1+\left(\frac{\delta y}{\delta x}\right)^{2}} \delta x
$$

\subsection{Fracture of Tissue}

The second key aspect of the infusion kit insertion scenario is the fracture of tissue. Using needles to penetrate skin has been used in medicine for delivery of drugs and increasingly minimally invasive surgery. There generally has not been a need to establish the critical conditions for the penetration of skin, needles will penetrate skin easily by hand. However, to establish possible failure criterion and device design requirements a more complete understanding of the fracture of tissue is required.

In classical fracture mechanics there are three types of fracture modes: Mode I, II and III [49]-[51]. Figure 10 shows an illustration of the three modes; Mode I is plane strain, Mode II is 
plane stress and Mode III is plane shear. In general when designing using Linear Fracture Mechanics, only Mode 1 fracture is considered; Shukla states that over $90 \%$ of all engineering design problems involving fracture are Mode I [51].
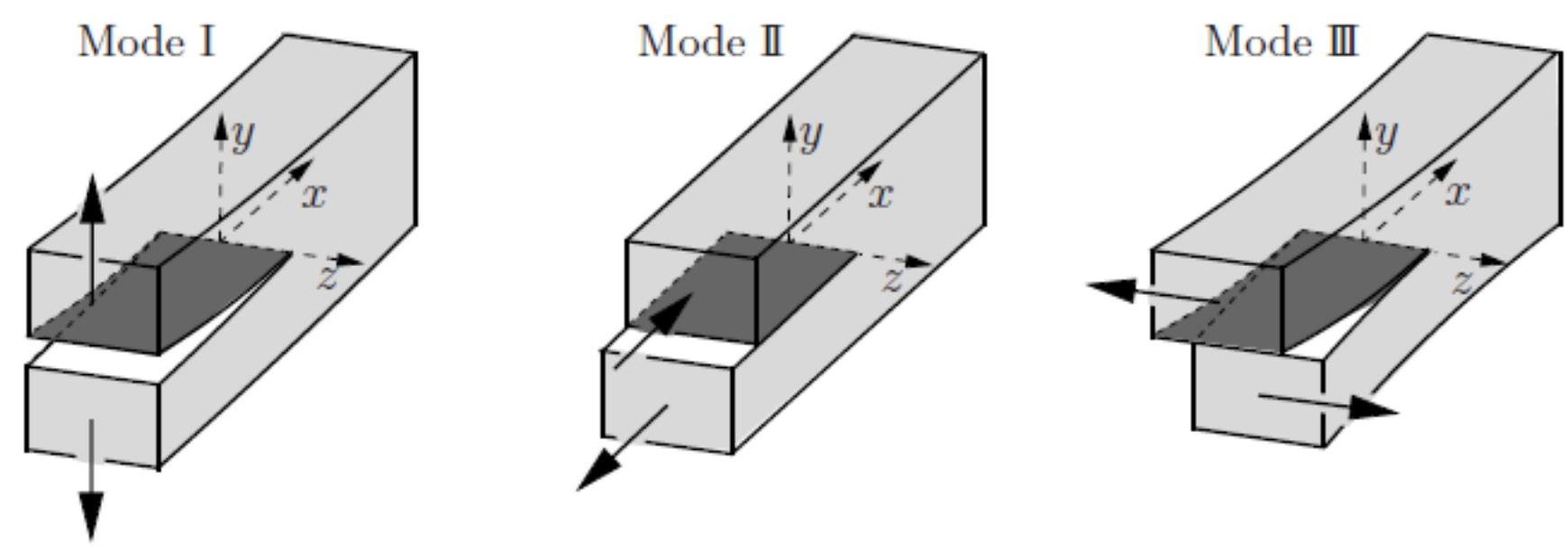

Figure 10: Fracture modes [50]

The tip region of a crack creates stress intensities in the material; the general equation is given by Equation 17. Where $\sigma$ is the applied stress, $a$ is the crack length and $C$ is a geometric constant, empirically derived based on the crack type and geometry. At some critical intensity, called $K_{I C}$ for Mode I loading, the crack propagates further into the material.

$$
K=C \sigma \sqrt{\pi a}
$$




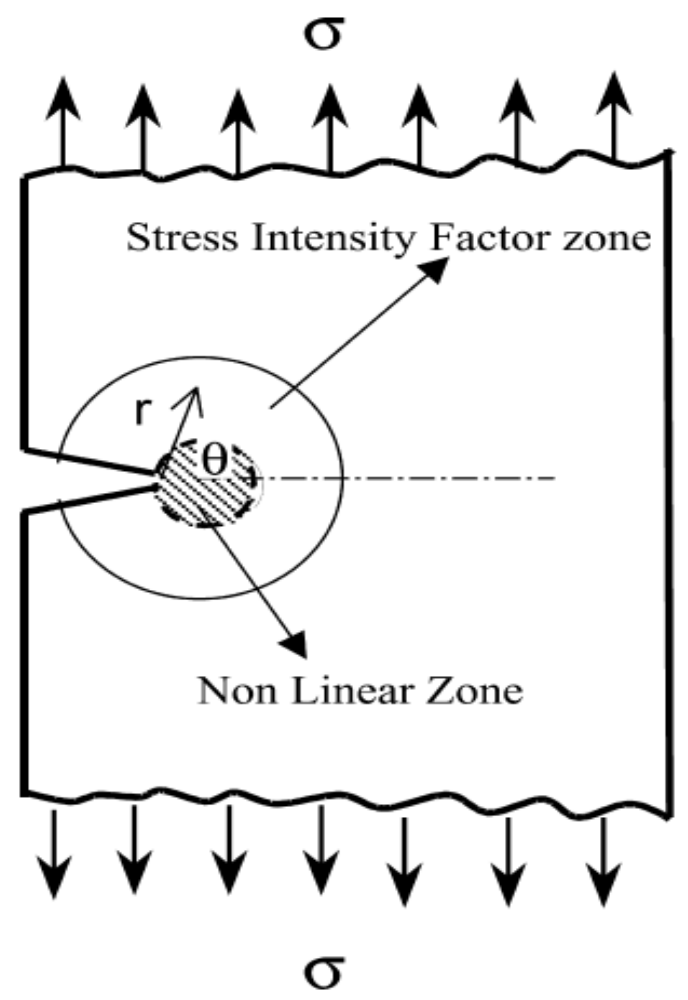

Figure 11: Definition of stress intensity zone [51]
There exists a zone around the crack tip that experiences this stress intensity; further away from the tip the stress in the material is the applied stress. A representation of this is given in Figure 11. The extent of this zone is not well defined; linear fracture mechanics is only concerned with the potential for crack propagation, not for fully defining the stress state that exists within the material. As will be discussed in more detail, a definition of this zone is critical for the scenario of a needle penetrating the skin surface. An estimation of the stress within the field is given by Equations 18 and 19 [50], [51]; where $\theta$ and $r$ are defined in Figure 11 and $K$ is defined in Equation 17.

$$
\begin{aligned}
& \sigma_{x}=\frac{K}{\sqrt{2 \pi r}} \cos \frac{\theta}{2}\left[1-\sin \frac{\theta}{2} \sin \frac{3 \theta}{2}\right] \\
& \sigma_{y}=\frac{K}{\sqrt{2 \pi r}} \cos \frac{\theta}{2}\left[1+\sin \frac{\theta}{2} \sin \frac{3 \theta}{2}\right]
\end{aligned}
$$

If an angle of $0^{\circ}$ is assumed, the cosine and sine terms become 1 and 0 respectively.

Substituting Equation 17 into either Equation 18 or 19 and setting the critical stress equal to the applied stress, the maximum radius of this stress affected zone becomes $r=\frac{c^{2} a}{2}$. In the case of an infinite plate with a center crack the geometry constant is approximately 1 [49]. This makes the maximum extent of the stress affected zone equal to approximately half the crack length.

The needle is assumed to create a line crack equal to its diameter as it fractures the tissue; to accommodate the entire circumference of the needle the tissue must deform. The energy 
required to do this deformation is dependant on the strain which is a function of the original unstrained geometry. If it is assumed that the only zone to experience deformation is the fracture stress intensity zone, then the strain becomes approximately $-100 \%$ which is not physically possible or realistic. Thus, using the stress intensity factor zone is not feasible for estimating the amount of strain energy required to deform the tissue to allow passage of the needle. An alternate definition of the strain zone will be developed in section 1.10 .

\subsection{Previous Work in Tissue Fracture}

Previous work in the fracture of tissue has been generally focused on predicting the needle trajectory to better control robotic and minimally invasive surgery or developing other methods to reduce the force required to fracture tissue. While derivations of the fracture and strain energy have been done, there has not been any concentrated effort into determining what effect skin tension, needle diameter and speed of insertion have on the fracture properties.

Work has been done to determine the force required to pierce human skin for use in the criminal forensics field. O'Callaghan et al. and Ni Annaidh et al. both performed experiments using knives and other common murder weapons to determine what factors influence the amount of force required to penetrate skin [52], [53]. Their results showed that the "sharpness" of the instrument greatly affects the amount of force required to penetrate skin. The results also showed that the surface layer of skin has greater resistance to penetration than the underlying fatty layers, this remains true until muscle tissue is encountered where the resistance increases.

Nature also provides for an abundance of structures designed to pierce skin: barbs, stingers and defensive quills. These structures are naturally formed and could provide insight into how to minimize the insertion force. The stinger of a honeybee has been studied by Ling et 
al. extensively [54], [55] and the proboscis of a mosquito by Aoyagi et al. and Kim et al. [56], [57]. Their results suggest vibration can reduce the insertion force and the complex microstructure of the stinger and proboscis also aids in the insertion process. The results, while promising, are too complex to incorporate into a mass-produced infusion kit. Experiments into more practical methods of lower insertion force have also been done by Chu et al. [58]. Their experiment compared a bare needle to metallic glass coated needles; their results found a reduction in peak force with the coated needles versus bare.

Work into controlling and predicting the trajectory of needles as they travel through a tissue is important for robotic surgery. Misra et al. postulated that the pressure differential on a bevelled needle is the primary mechanism which causes needle deflection during insertion [59]. Using scaled up models of bevel needles, they inserted a range of needle tips into synthetic elastic gels to simulate tissue. Their results showed an influence of needle diameter and bevel angle on the radius of curvature on the needle during insertion. Liu et al. performed similar experiments, except they investigated the effect muscle fiber direction has on the maximum cutting force [60]. Their results showed that inserting a needle perpendicular to the fiber direction provided the lowest cutting force, while inserting the needle parallel to the fibers resulted in the largest peak force. Naemura et al. and found little change with changes in Young's Modulus and the insertion force [61].

Heverly and Dupont studied the influence of two insertion speeds on the cutting force during insertion [62]. Their results showed a decrease in peak force at higher insertion speeds. Mahvash and Dupont also performed similar experiments using a greater range of insertion speeds [63]. Their results agreed with those of Heverly where higher speeds produced a lower peak force. However, their results also suggest that at some velocity the peak force begins to 
plateau and remain relatively unchanged as insertion velocity increases. Similar work was also done by Gao et al. and Casanova et al. [48], [64].

Shergold and Fleck investigated and created a finite element model to calculate the amount of work required to punch through tissue using flat and sharp punches [65], [66]. Their material model used an Ogden material model and assumed a constant volume annulus. Their model could only be solved numerically which makes it less useful for the analysis presented below; however, the analysis draws heavily from their initial model set up. Finite element models have also been created by Oldfield and Han [67], [68]. These models are highly situational which makes mass application difficult. Section 1.10 will present an analytical solution for the derivation of a fracture coefficient of tissue.

\subsection{Mathematical Model for the Fracture of Tissue}

The equation for the fracture work is given in Equation 20; where $J_{I C}$ is the fracture toughness of the tissue, $\delta A$ is the change in crack area along the direction of the needle trajectory.

$$
\delta W_{\text {fracture }}=J_{I C} \delta A
$$

It is commonly assumed that tissue is incompressible, which would result in the deformation work being zero. This assumption is valid for certain analyses but does not hold for the deformation of the skin to accommodate the needle shaft. The applied strain is not quasistatic; therefore, viscoelastic resistance will need to be overcome contributing to the net work of the system. This can be thought of as an elastic deformation. There is strain energy associated with the widening of the crack. If it is assumed that this strain is purely elastic, the work required to deform the skin is given by the volumetric integral in Equation 21 . The stresses and strains are in the plane perpendicular to the crack path. 


$$
\delta U=\int_{V o l} \sigma_{i j} \epsilon_{i j}
$$

Michell derived some equations for relating a point force to the perpendicular stresses in an elastic solid [69]. These equations were derived without taking into account stress concentrations caused by a fracture or the local regions of zero stress around the crack perimeter. The Cartesian field stress from Michell is given in Equation 22; where $\mathrm{F}$ is the applied load, $\theta$ is the radial angle, $\lambda$ and $\mu$ are the first and second Lamé material parameters and $r$ is the radial distance from the center.

$$
\sigma_{x}=\sigma_{y}=\frac{\mu}{\lambda+2 \mu} \frac{F}{4 \pi} \frac{\cos \theta}{r^{2}}
$$

It is apparent as the radius approaches zero the stress becomes infinite which is unrealistic. The equation is equally meaningless for large values of radius. A defined zone of stress is required to make the equation meaningful. Equally, the field strain also requires a reference affected zone to compute.

Shergold and Fleck derived a method for calculating the strain energy for this scenario using an Ogden model [66]. However, this solution needs to be calculated analytically; further the model requires some assumptions about the size of the crack, denoted as a, versus the radius of the needle, denoted as R. For low values of a/R the strain energy dominates the fracture work however at higher values $(\mathrm{a} / \mathrm{R} \approx 4)$ the energy required for crack propagation is the dominant term. Based on the pictures provided by Shergold and Fleck [65], it appears that the fracture is only as large as required to accommodate the punch.

It is required to define a zone in which the strains and stresses are applied; for simplicity it will be assumed that these zones are equal. As detailed in section 1.8 this zone cannot be the stress intensity zone of a crack tip or the strain would be $-100 \%$ which does not make physical 
sense. If it is assumed that the only contributors to the net work are the fracture and the elastic deformation the system work equation becomes Equation 23.

$$
\delta W_{\text {sys }}=\delta W_{\text {fracture }}+\delta W_{\text {elastic }}
$$

If it is then assumed that the stress and strain affected volume is equal and constant, and the stress and strain fields are radial, the elastic work integral from Equation 21 becomes Equation 24.

$$
W_{\text {elastic }}=\sigma_{r} \epsilon_{r} V
$$

Since the assumption that the stress is radially constant, the cosine term may be dropped from Equation 22 and the stress equation becomes Equation 25.

$$
\sigma_{r}=\frac{\mu}{\lambda+2 \mu} \frac{F}{4 \pi r^{2}}
$$

It will then be assumed the radius of the affected zone is not large, and it can be assumed that the interface stress of the needle and skin $\left(r=r_{\text {needle }}\right)$ is constant through the zone.

The definition of plane strain is Equation 26. The skin surface will deform from the closed crack to an annulus of the same radius as the needle to allow the needle to pass.

$$
\epsilon=\frac{\Delta A}{A}=\frac{\pi r_{\text {needle }}^{2}}{A}
$$

Substituting Equation 25 and 26 into Equation 24 and rewriting the volume into the product of the stress and strain zone and the change in depth; the elastic work then becomes Equation 27.

$$
W_{\text {elastic }}=F_{\text {elastic }} \delta z=\frac{\mu}{\lambda+2 \mu} \frac{F_{\text {total }}}{4 \pi r_{\text {needle }}^{2}} \frac{\Delta A}{A} A \delta z \quad \text { where } \Delta A=\pi r_{\text {needle }}^{2}
$$

Since the stress within the affected zone was assumed to be equal to the stress at the outer radius of the needle eliminating the need to define the planar area of the affected zone; further, it now also becomes independent of the thickness of the specimen and the radius of the needle. The 
reaction force resulting for the elastic deformation from the crack to the full diameter of the needle is given in Equation 28.

$$
F_{\text {elastic }}=\frac{\mu}{\lambda+2 \mu} \frac{F_{\text {total }}}{4}
$$

The Lamé material parameters, $\lambda$ and $\mu$ are given by Equations 29 and 30 , where $\mathrm{E}$ is the Young's Modulus and $v$ is the Poisson's ratio.

$$
\begin{gathered}
\mu=\frac{E}{2(1+v)} \\
\lambda=\frac{E v}{(1+v)(1-2 v)}
\end{gathered}
$$

Equation 31 gives the factored first term of Equation 28.

$$
\frac{\mu}{\lambda+2 \mu}=\frac{1}{2\left(\frac{v}{1-2 v}+1\right)}
$$

The work done by the fracture process can equally be made independent of the depth of penetration by rewriting Equation 20 into Equation 32 resulting in Equation 33.

$$
\begin{gathered}
\delta W_{\text {fracture }}=F_{\text {fracture }} \delta z=J_{I C} \delta z a \\
F_{\text {fracture }}=J_{I C} a
\end{gathered}
$$

Combining Equations 28 and 33, the total reaction force from the deformation and fracture becomes Equation 34 .

$$
F_{\text {total }}=J_{I C} a+\frac{\mu}{\lambda+2 \mu} \frac{F_{\text {total }}}{4}
$$

For an incompressible material $(v=0.5)$ the $\lambda$ term in Equation 30 becomes infinite. Other literature has given values of 0.36 [40] and 0.45 [48]. A Poisson's ratio of 0.36 gives a value of approximately 1/4.5 for Equation 31 and a Poisson's ratio of 0.45 gives a value of 1/11. Since skin tissue is commonly assumed to be incompressible a Poisson's ratio value close 
to 0.5 is more appropriate. However, even assuming a ratio value of 0.36 , Equation 28 reduces to $F_{\text {elastic }}=\frac{F_{\text {total }}}{18}$. Since this value will be small compared to the value of the total force this term will be neglected. The fracture toughness is then given by Equation 35 .

$$
J_{I C}=\frac{F_{\text {total }}}{a}
$$

\subsection{Damage to the Cannula}

The original purpose of the research was to establish damage and failure criteria of the insertion process of a spring loaded insulin infusion kit. Since much of the necessary background knowledge required to properly model the insertion was incomplete, the damage and failure criteria were not defined by this thesis. However, the basics of how the system was to be modeled is presented in this section.

The needle and cannula are essentially independent of each other; their connection point is at the far end of the system. After the needle fractures the skin surface, the spring drives the cannula into contact with the surface of the skin. The skin must deform to accommodate the cannula similar to what is described in section 1.9. The skin surface imparts a reaction force onto the canula opposite to the direction of the spring force. This combination of forces sets up a classic buckling scenario with a fixed end and guided end [49]. The complicating factors are the central needle, which acts as an independent support, and the hollow cylinder shape of the cannula. The first factor is a unique complication, there is simply no comparable real world engineering scenario in which an outer column is subjected to a buckling load while an inner column is not. This means the lateral deflection that occurs in classical buckling is limited by a stiff central support which complicates the modelling of the buckling scenario. The shape of the cannula is also not classic thick wall buckling; it would need to be modelled as a shell as 
described by the works of Timoshenko and Leissa [70], [71]. Preliminary testing found that the cannula would "crumple" approximately a quarter of the length from the fixed end. This behaviour would have to be described by an amalgamation of buckling theory and shell theory.

Finally, the amount of load applied to both ends of the cannula is time dependant but independent of each other. The spring would be assumed to be a linear Hookean spring, but the skin surface would be reacting as it was indented. The work presented in the following sections would allow for a method of estimating the amount of deflection and thus reaction force the cannula would experience. The effect of the central fracture caused by the needle would also have to be reconciled with the reaction caused by indentation.

\subsection{Research Goals}

There are two main goals of the research presented here. The first goal is to quantify the effect that skin tension, indentation velocity and tip geometry have on the mechanical response of skin tissue. The second is to quantify the effect skin tension, insertion velocity and needle diameter have on the fracture properties of skin tissue. Due to research ethics and supply considerations human tissue has been substituted with porcine skin. There has been no literature found directly comparing the properties of human and porcine skin tissue, but investigators have previously used the same material models to describe the behaviour of both types of tissues. The results obtained will be compared to others found in literature, but, the primary objective is to identify any trends and explain them in the context of the testing method. The three independent variables were chosen because it is reasonable to assume that they would impact mechanical and fracture properties. Increasing the skin tension should increase the reaction force during indentation, pretensioning the collagen fibers would eliminate or reduce the "toe" region as 
described in sections 1.2 and 1.3. This would shift the response into the second region which is associated with a much larger modulus which would result in larger reaction forces. This, however, should not affect the fracture properties to any real degree as breaking the collagen linkages require a fixed amount of energy. As a viscoelastic material, skin tissue would, by definition, be affected by the strain rate, which will be controlled by the indentation and insertion velocities. Increasing velocity should result in higher reaction force and a lower fracture coefficient. Tip geometry and needle diameter should affect the mechanical and fracture response as both parameters are integral to the definition of the stress and strain state of the system.

\subsection{Thesis Structure}

Section 2 contains the work done on determining the mechanical properties of porcine skin subjected to an indentation. Section 2.1 contains a description of the differences between the document submitted to be published and the thesis. Section 3 contains the work done on determining the fracture properties of porcine skin. Section 3.1 contains a description of the differences between the document submitted to be published and the thesis. Section 4 contains supplementary discussion and the limitations and sources of error of the thesis work. Section 5 contains the thesis conclusion. Six appendices are included containing supplementary figures, descriptions of the instrumentation and apparatus, the calibrations done, the codes used, an example of the collected data and the calculated results and the instrumentation data sheets. 


\section{Chapter 2: The Effect of Skin Tension, Indentation Speed and Tip Contact}

\section{Area on the Shear Modulus and Viscoelastic Relaxation Periods of Porcine}

\section{Tissue}

\subsection{Differences from the Journal Submission Version}

The introduction section of the journal submission version has been omitted from the thesis. The relevant theory has been developed in Sections 1.1 through 1.7. The mathematical model has been kept in a condensed version, for reference, in Section 2.2. Numbering of equations has reset; numbering of figures and tables has not.

\subsection{Experimental Model}

The stress in a viscoelastic material is given by Equation 1 [44]; it has been assumed that planar isotropy does not have an effect on the scale at which this analysis is taking place. Where $\sigma$ is the stress, $E$ is the modulus of the material, $\tau$ is the integration variable, $t$ is the time and $\epsilon$ is the strain.

$$
\sigma_{i j}=\int_{-\infty}^{t} E_{i j k l}(t-\tau) \frac{\delta \epsilon_{k l}(\tau)}{\delta \tau} \delta \tau
$$

If the strain is assumed to be instantaneously applied by a step function, then the strain becomes constant for $\mathrm{t}>0$ and 0 for $\mathrm{t}<0$. A general Maxwell model is used, spring constants and damping coefficients are required, and an assumption of uniaxial stress is applied. The strain can be factored out of the integral in Equation 1; the remainder of the integration is evaluated, resulting in the uniaxial stress state, Equation 2.

$$
\sigma=\epsilon\left(E_{\infty}+E_{1} e^{-\frac{t}{\tau_{1}}}+E_{2} e^{-\frac{t}{\tau_{2}}}\right)
$$


There has been some discussion whether $E_{1}$ and $E_{2}$ are physically meaningful or if they are simply fitting terms [47]. This model assumes that they are fitting terms, thus does not constrain them to be solely positive (which would be the case if they were physical moduli). There are two meaningful modulus values that are derived from this equation: $E_{0}$ and $E_{\infty}$. $E_{0}$ is the summation of $E_{\infty}, E_{1}$ and $E_{2}$ at $\mathrm{t}=0$ and represents the initial viscoelastic response to the applied strain. $E_{\infty}$ is the long-term elastic modulus of the material which could be viewed as an equivalent Young's or shear Modulus. The two relaxation periods $\tau_{1}$ and $\tau_{2}$ are interpreted to be the short and long-term stress relaxation periods of the material. Since force was recorded during experimentation the stress in Equation 2 is decomposed into Equation 3.

$$
F=A_{\text {tip }} \epsilon_{\text {surface }}\left(E_{\infty}+E_{1} e^{-\frac{t}{\tau_{1}}}+E_{2} e^{-\frac{t}{\tau_{2}}}\right)
$$

\subsubsection{Estimation of Strain and Tip/Skin Contact Area}

The estimation of the value of induced strain is required to complete this analysis; there exists a strain affected zone within the sample. The depth and extent of the strain affected zone is not readily apparent. Gao et al. performed experiments using needles and simulated tissue [48]. They found the radius of the indented zone was approximately 1.67 of the needle depth at puncture of the skin. This ratio is taken to be the maximum extent of the strain affected zone from the indenter's outer radius. With a fixed, assumed indenter depth of $2.5 \mathrm{~mm}$ in all tests that zone is $R=4.175 \mathrm{~mm}$ from the tip in the conical cases and from the outer edge in the cylindrical cases. This zone is assumed to only vary with the type of tip, not with test configuration or any other external factor.

Skin is commonly assumed to be incompressible; with this assumption, the deformation of the skin is simplified to the change in length of a 2-D beam, with the unstrained state being the 
radius of the strain affected zone.

In the case of the

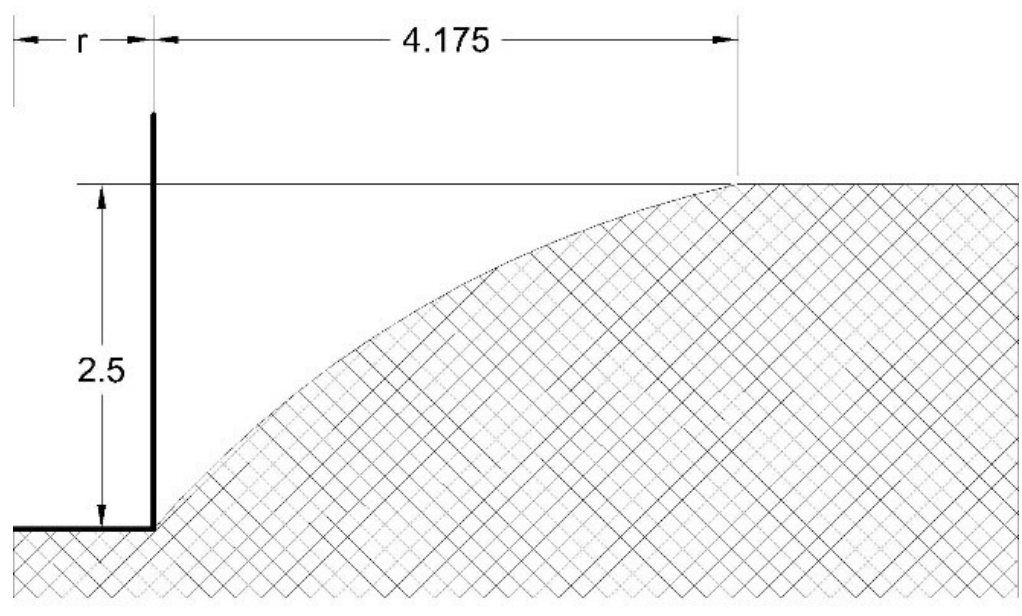

cylindrical indenters it can be assumed that the strain affected zone will extend from the edge of the indenter face. The strain affected zone will become the indenter radius plus the assumed zone of $4.175 \mathrm{~mm}$. Figure 12 shows the model set up.

Figure 12: Geometry of cylindrical indenter tip - skin interaction Sneddon's solution to indentation in an elastic half space gives an estimate of the deformed surface is shown in Equation 4 [37]. Where $D$ is the indentation depth, $a$ is the indenter radius and $p$ is the radial distance.

$$
u_{z}=\frac{2 D}{\pi} \sin ^{-1}\left(\frac{a}{p}\right), p>a
$$

The strain estimate of the

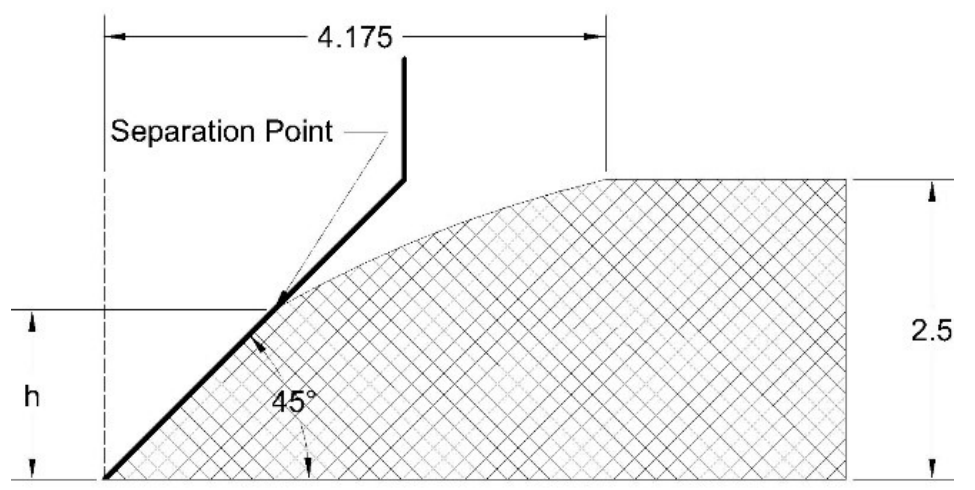

Figure 13: Geometry of conical indenter tip - skin interaction conical indenters is derived in a similar manner. Since the conical indenters end in a point instead of a flat face; the strain affected zone will equal $R$ in all cases. The tip will have full contact for some height from the tip $h$ before the surface will no longer 
have contact with the indenter. Figure 13 illustrates how the skin interacts with the indenter.

The radius at which the surface detaches from the surface of the cone is given by Equations 5 and 6 . Figure 13 shows the geometry of $a$ and $h$. Where $\alpha$ is the outside angle of the tip, labeled in Figure 13 as $45^{\circ}$.

$$
\begin{gathered}
a=\frac{2 D}{\pi \tan \alpha} \\
h=\frac{\sin (90-\alpha)}{a}
\end{gathered}
$$

The deformed surface is then given by Equation 7 .

$$
u_{z}=\frac{2 D}{\pi a}\left(a \sin ^{-1}\left(\frac{a}{p}\right)-p+\sqrt{\left(p^{2}-a^{2}\right.}\right), p>a
$$

The boundary condition of $u=0$ at $p=R+a$ for the cylindrical indenter and at $p=R$ for the conical indenter results in a mathematical discontinuity $\left(u_{z} \neq 0\right)$ at those points. Without direct measurement of the surface deformation, this limitation is unavoidable. The assumption of a linear elastic material is part of the reason this discontinuity exists.

The length of these curves is determined by numerical integration with Equation 8 . The strain is calculated from the difference between the undeformed surface and the length of the deformed surface.

$$
L=\int_{a}^{b} \sqrt{1+\left(\frac{\delta y}{\delta x}\right)^{2}} \delta x
$$

\subsection{Methods and Materials}

Indentation testing was performed using fresh porcine belly skin sourced from a local abattoir. The sample was subdivided into squares approximately $175 \mathrm{~mm}$ by $175 \mathrm{~mm}$, these were 
then trimmed to a thickness of approximately $10 \mathrm{~mm}$ to $12 \mathrm{~mm}$ with emphasis on ensuring constant thickness and removing the muscle tissue where possible. The sample was then sutured and mounted to brackets that, when tightened, provided tension in the in-plane directions. An SType load cell (Interface Inc. SM-25, Scottsdale, Arizona) was mounted along each axis measuring the applied tension. Medical ballistics gel (Humimic Medical \#0 Medical Gel, Greenville, South Carolina) was placed underneath the sample to simulate a muscle layer. Figure 14 shows the experimental set up.

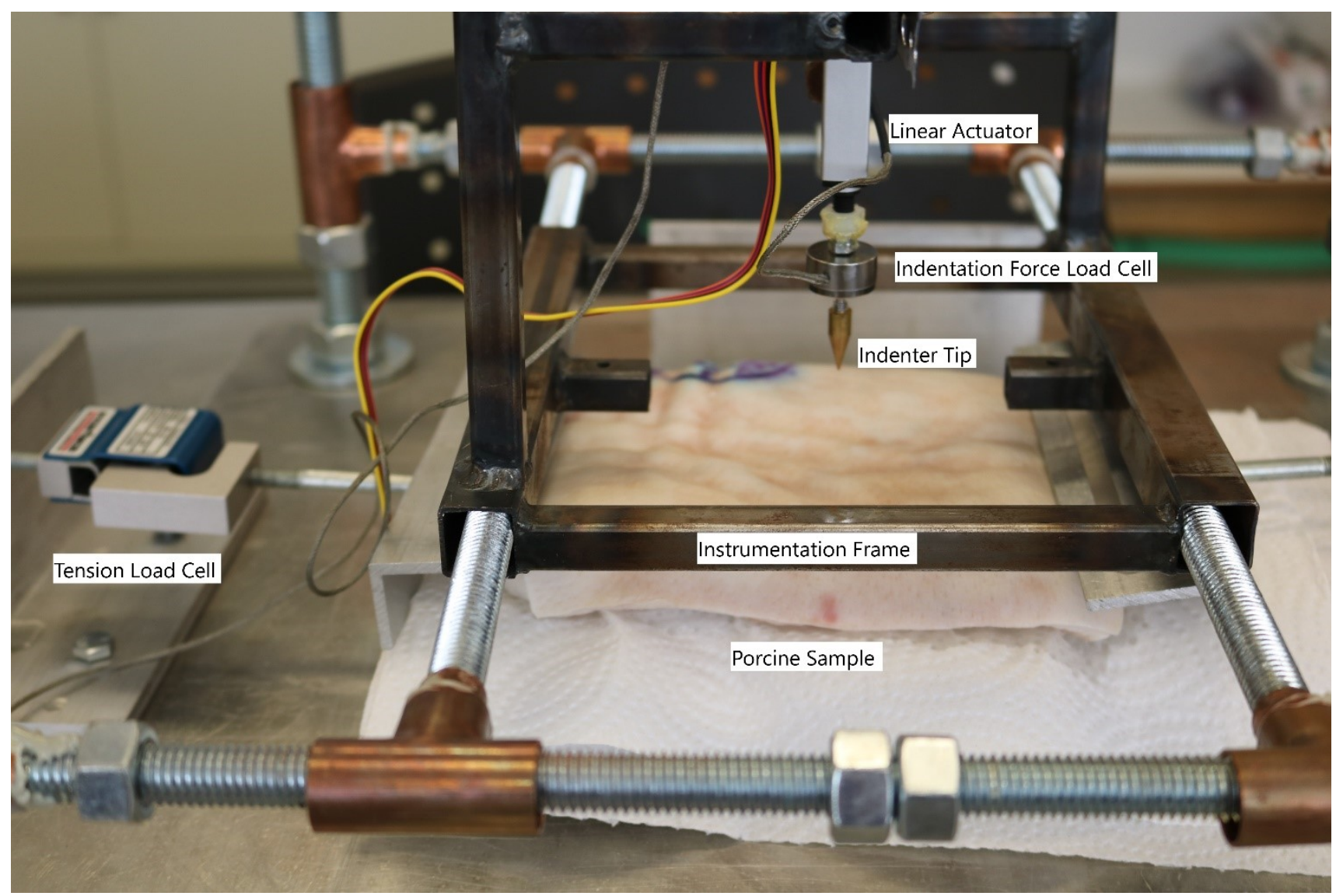

Figure 14: Experimental apparatus

A geared drive train linear actuator (Actuonix Motion Devices Inc. L12-P Micro Saanichton, British Columbia) was used to extend the indenter tips to the skin surface. For testing seven speeds settings were chosen and calibrated using the on-board variable resistor. The speeds were $0.0116 \mathrm{~m} / \mathrm{s}, 0.0162 \mathrm{~m} / \mathrm{s}, 0.0193 \mathrm{~m} / \mathrm{s}, 0.0199 \mathrm{~m} / \mathrm{s}, 0.0236 \mathrm{~m} / \mathrm{s}, 0.0243 \mathrm{~m} / \mathrm{s}$ and 0.0260 
$\mathrm{m} / \mathrm{s}$.

Seven separate indenter tip configurations were used, a $30^{\circ}, 45^{\circ}, 60^{\circ}$ and $75^{\circ}$ cone; and a $1.23 \mathrm{~mm}, 2.63 \mathrm{~mm}$ and $4.50 \mathrm{~mm}$ diameter cylinder. Figure 15 shows example geometry of the indenter tip. The reaction force on the tips was measured by an inline load cell (Omega Engineering Inc. LCMFL-20N, Stamford, Connecticut) which was attached to the actuator.

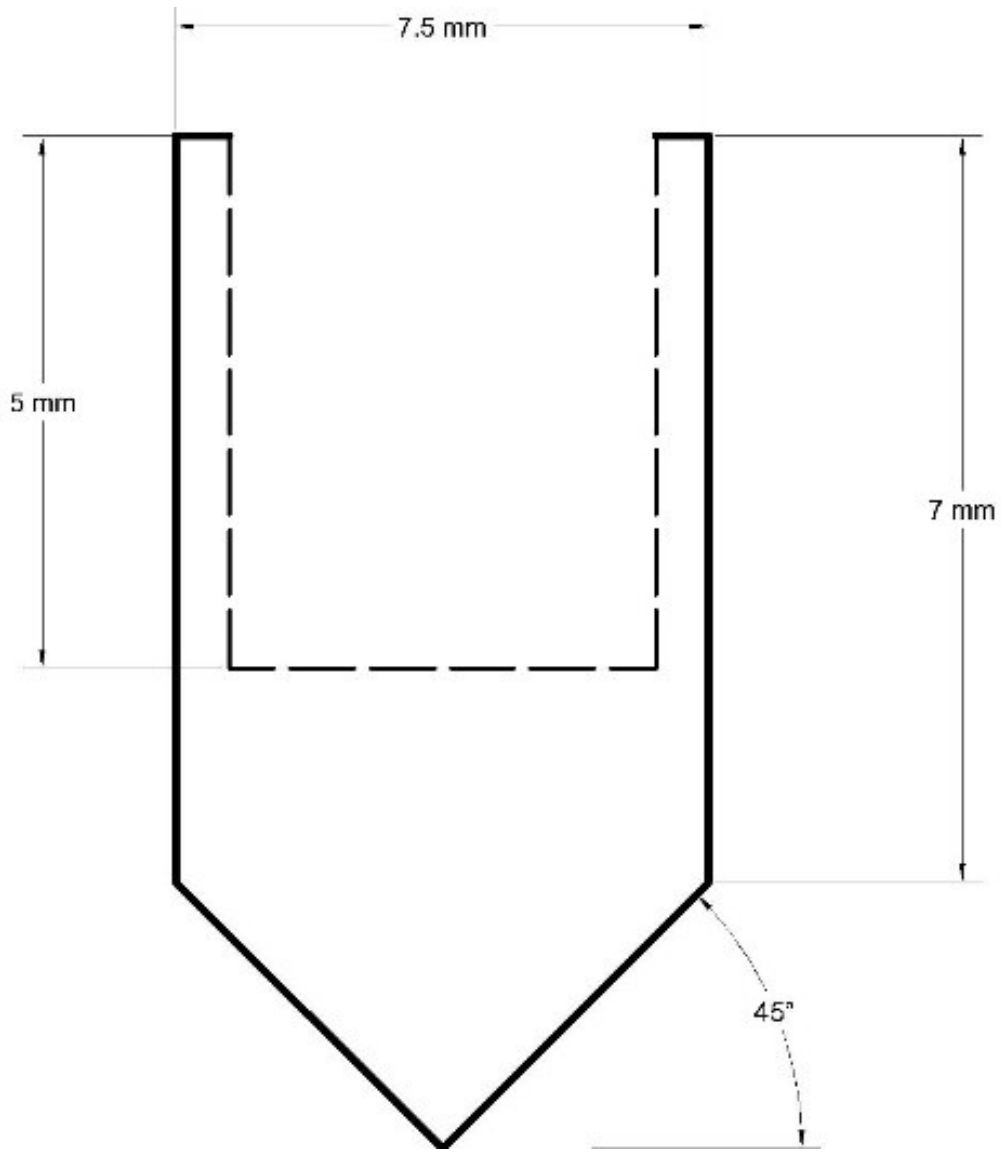

Figure 15: Example tip geometry

The prepared pork samples were wrapped in paper towel, soaked in water, and refrigerated for a minimum of 2 hours. The samples were then brought up to room temperature for approximately 1 hour, still wrapped in the paper towel and then sutured into the clamps. The sample was tensioned in the transverse plane and allowed to relax for approximately 10 minutes before testing commenced. It was found during calibration that after 10 minutes the change in 
recorded tension values for the test duration were minimal. Indentation tests were done on 9 sites, arranged in a 3 by 3 grid, a minimum of 60 millimeters from the edge of sample.

Calibration testing showed no change in test data gathered at the beginning versus the end of the test period. Data collection and linear actuator control was done with an Arduino Due system connected to the Actuonix Linear Actuator Control Board. The tip of the indenter was set to travel to a displacement of 2.5 millimeters below the surface of the sample. The displacement was then held for approximately 9 seconds. Total time from beginning of suturing to end of testing was approximately 1.5 hours; care was taken to ensure that the pork sample remained hydrated during testing.

The test was repeated for each of the seven tip configurations at the same speed on the single sample. The test was repeated twice more on different samples with different tension values. The three trials were then repeated with the six different speeds.

Equation 3 was used to fit the experimental force profile using a non-linear least squares fit tool in Matlab R2017b (Mathworks Inc., Natick, Massachusetts).

Statistical analysis was done using Stata SE 15.1 (StataCorp LLC, College Station, Texas). The effect of skin tension, indentation speed and indenter tip geometry on the maximum force and the four viscoelastic parameters was evaluated using step-wise linear regression. An independent variable was removed if its effect was not significant and the regression was repeated until no more variables were removed. The strength of the effects of independent variables on the dependent variable were compared with beta, the standardized regression coefficient; beta measures the change in the dependent variable due to a change in the independent variable, in multiples of the standard deviations of the respective variables. Unlike the regression coefficient, beta allows comparison of variables of different units and with 
different orders of magnitude. The higher the beta value, the greater the effect the independent variable has on the dependent variable. Beta values are not reported for insignificant variables. The overall strength of the regression was also assessed with the coefficient of determination $\left(\mathrm{R}^{2}\right)$.

\subsection{Results}

Table 2 shows the calculated contact surface areas and strains for each indenter tip.

Table 2: Indenter tip contact surface area and induced strain results assuming an indentation of $2.5 \mathrm{~mm}$

\begin{tabular}{|l|l|l|}
\hline Tip & Contact Surface Area $\mathrm{mm}^{2}$ & Strain \\
\hline $1.23 \mathrm{~mm}$ diameter cylinder & 1.19 & 0.276 \\
\hline $2.63 \mathrm{~mm}$ diameter cylinder & 5.43 & 0.185 \\
\hline $4.50 \mathrm{~mm}$ diameter cylinder & 15.9 & 0.125 \\
\hline 30 Degree Cone & 27.6 & 0.114 \\
\hline 45 Degree Cone & 11.3 & 0.182 \\
\hline 60 Degree Cone & 5.31 & 0.260 \\
\hline 75 Degree Cone & 2.21 & 0.361 \\
\hline
\end{tabular}

Figure 16 shows an example of the experimental force data with the curve fit. The calculated curve fits underestimated the peak region but predicted the long-term response well. 


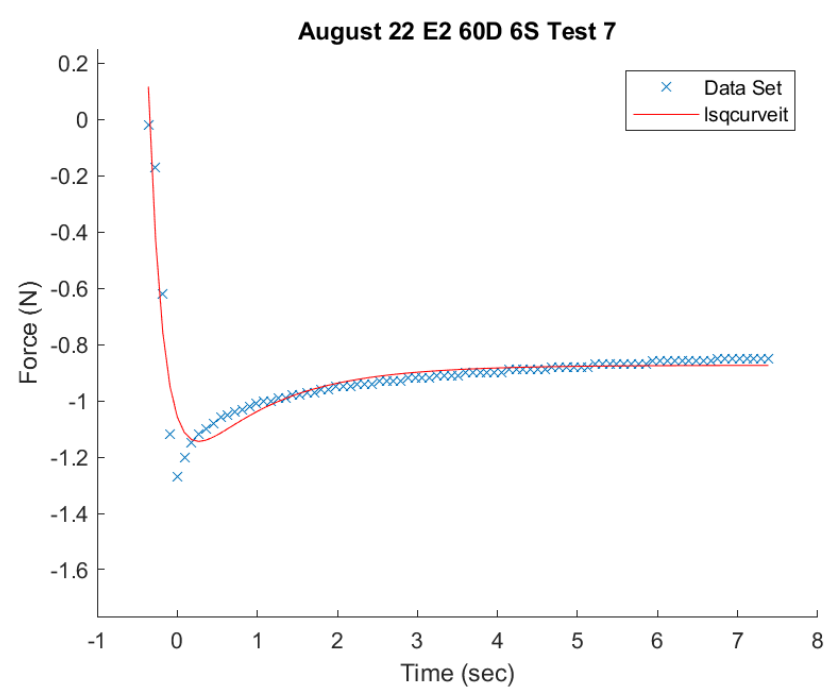

Figure 16: Example experimental results and fit as per Equation 3

\subsubsection{Force Results}

The average maximum force recorded of all the specimens was $-1.76 \mathrm{~N}$; the standard deviation was $0.66 \mathrm{~N}(\mathrm{n}=1332)$. The linear regression equation is given by Equation 9. The $\mathrm{R}^{2}$ value of the regression fit is 0.094 , indicating the variance is poorly explained by the two significant variables. Table 3 shows the experimental maximum recorded force coefficients from the statistical analysis. Force was measured as a negative value as it was a compressive force on the load cell. Speed and surface area significantly affect the maximum force $(p<0.05)$ while tension does not. The beta value for speed is an order of magnitude higher than surface area indicating it has a stronger effect on the change in maximum force. Figures 17,18 and 19 show the results of maximum force plotted against the three different independent variables.

$$
F_{\text {max }}=-41.76 * \text { Speed }-0.0054 * A_{\text {surface }}-0.89
$$


Table 3: Step-wise regression results of the maximum force. Indentation speed and indenter tip surface area were significant independent variables $(\mathbf{p}<\mathbf{0 . 0 5})$ applied surface area was not significant and was omitted from the final regression equation. The beta value of speed is an order of magnitude larger than surface area indicating speed has a greater effect on the maximum force. The $R^{2}$ value of 0.094 indicates that speed and surface area have a weak relationship to the maximum force.

\begin{tabular}{|l|l|l|l|l|l|}
\hline & Value & $\mathrm{p}$ & Lower 95\% confidence & Upper 95\% confidence & Beta \\
\hline Tension & -0.0059 & 0.18 & -0.014 & 0.0027 & \\
\hline Speed & -41.76 & 0.000 & -48.97 & -34.54 & -0.30 \\
\hline Surface Area & -0.0054 & 0.006 & -0.0093 & -0.0015 & -0.072 \\
\hline Constant & -0.89 & & -1.05 & -0.74 & \\
\hline
\end{tabular}

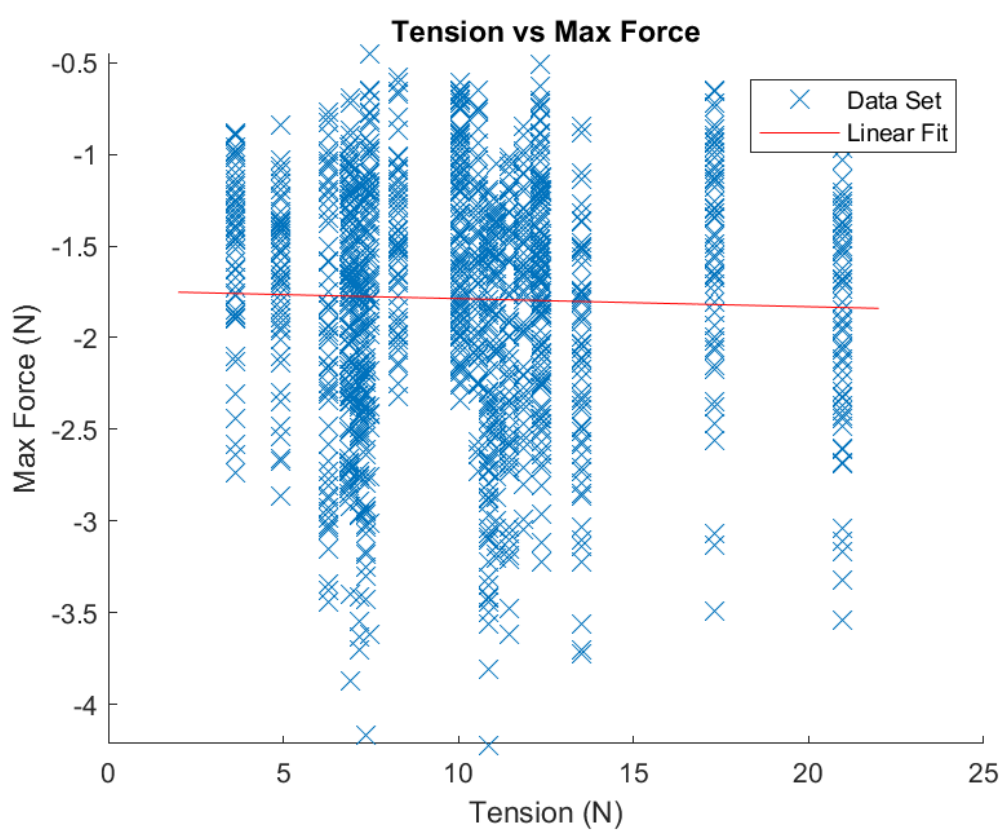

Figure 17: Measured maximum force plotted against applied tension; the red line shows the pair-wise relationship from a simple regression. Changes in tension were not found to be significant $(p=0.18)$ in affecting the maximum force. 


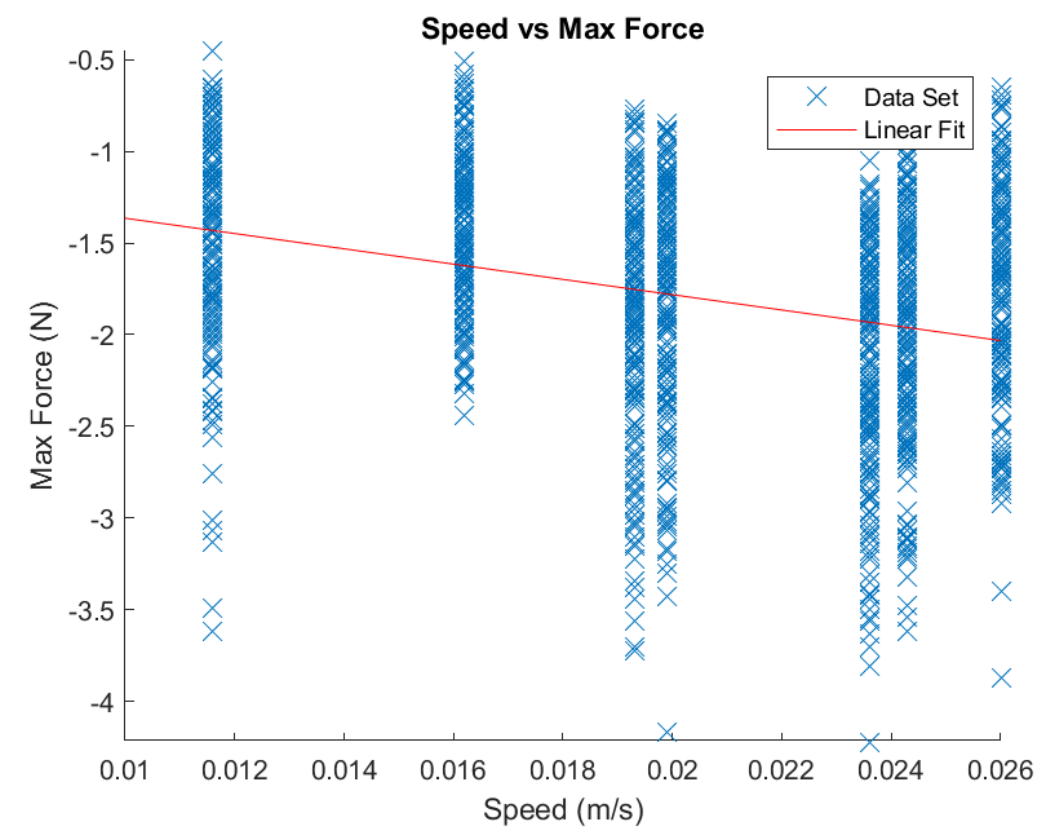

Figure 18: Measured maximum force plotted against indentation speed; the red line shows the pair-wise relationship from a simple regression. Changes in speed were significant $(p<0.05)$ in affecting the maximum force.

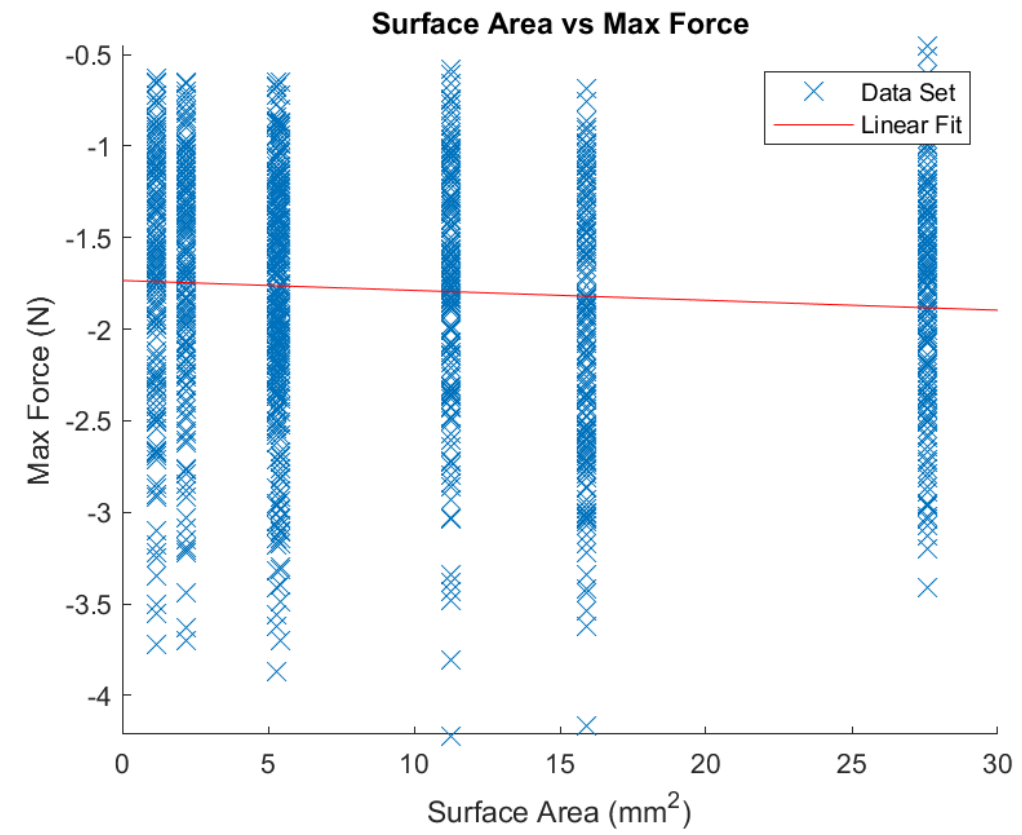

Figure 19: Measured maximum force plotted against indenter tip surface area; the red line shows the pairwise relationship from a simple regression. Changes in surface area were significant $(p<0.05)$ in affecting the maximum force. 


\subsubsection{Initial Modulus Results}

The average calculated initial modulus of all the specimens is $2.52 \mathrm{MPa}$; the standard deviation was $1.79 \mathrm{MPa}(\mathrm{n}=1332)$. Equation 10 gives the linear regression fit. Table 4 shows the calculated results of the initial modulus coefficients from the statistical analysis. This modulus is positive as the strain is taken to be negative in Equation 3. Tension, speed and surface area significantly affect the initial modulus $(\mathrm{p}<0.05)$. The $\mathrm{R}^{2}$ value of the regression fit is 0.65 indicating that the variance is well explained by changes in the three variables. Beta values for speed and surface area are similar with surface area having a larger effect on the initial modulus than speed. The beta value for tension is an order of magnitude smaller than that of speed and surface area indicating a weak effect compared to the other variables. Figures 20, 21 and 22 show the calculated initial modulus plotted against the three different independent variables.

$$
E_{0}=0.017 * F_{\text {tension }}+55.49 * \text { Speed }+0.16 * A_{\text {surface }}-0.38
$$

Table 4: Step-wise regression results of the initial modulus. Applied tension, indentation speed and indenter tip surface area were significant independent variables $(p<0.05)$. The beta values of surface area and speed are an order of magnitude larger than tension and the beta value of surface area is larger than that of speed. This indicates surface area has the greatest effect on the initial modulus followed by speed then tension. The $R^{2}$ value of 0.65 indicates that the independent variables explain the variance of the initial modulus well.

\begin{tabular}{|l|l|l|l|l|l|}
\hline & Value & $\mathrm{p}$ & Lower 95\% confidence & Upper 95\% confidence & Beta \\
\hline Tension & 0.017 & 0.021 & 0.0026 & 0.032 & 0.038 \\
\hline Speed & 55.49 & 0.000 & 43.22 & 67.76 & 0.14 \\
\hline Surface Area & 0.16 & 0.000 & 0.16 & 0.17 & 0.79 \\
\hline Constant & -0.38 & & -0.69 & -0.080 & \\
\hline
\end{tabular}




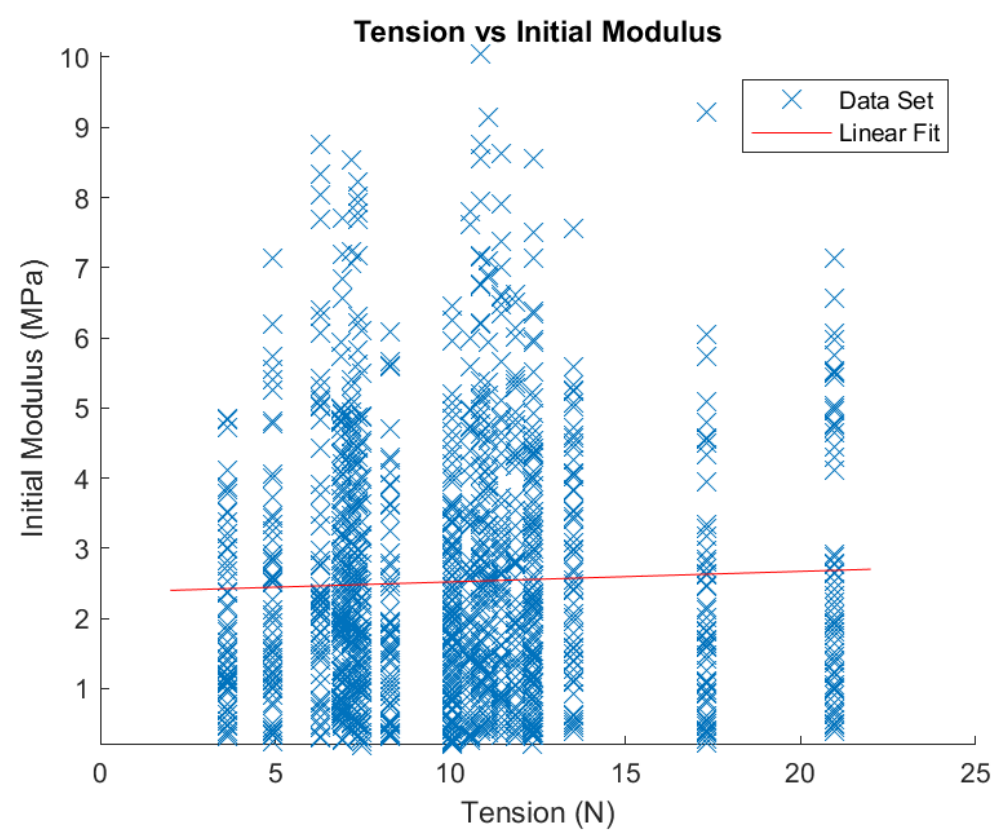

Figure 20: Calculated initial modulus plotted against applied tension; the red line shows the pair-wise relationship from a simple regression. Changes in tension were significant $(p<0.05)$ in affecting the initial modulus.

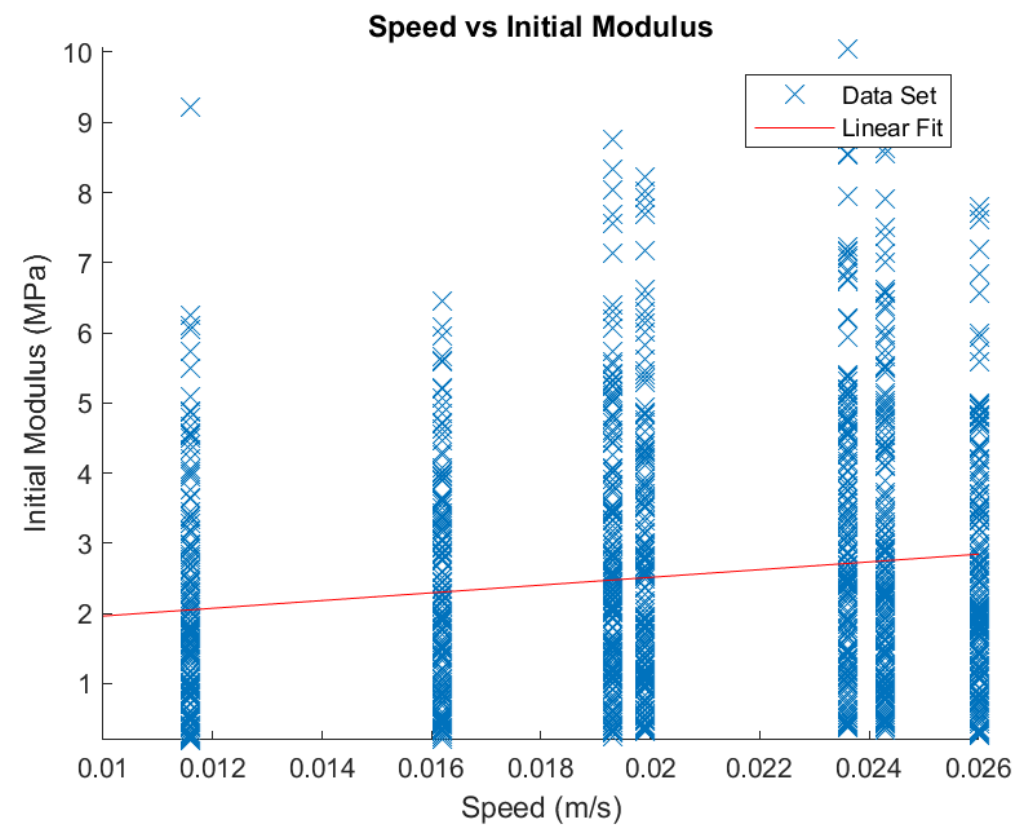

Figure 21: Calculated initial modulus plotted against indentation speed; the red line shows the pair-wise relationship from a simple regression. Changes in speed were significant $(p<0.05)$ in affecting the initial modulus. 


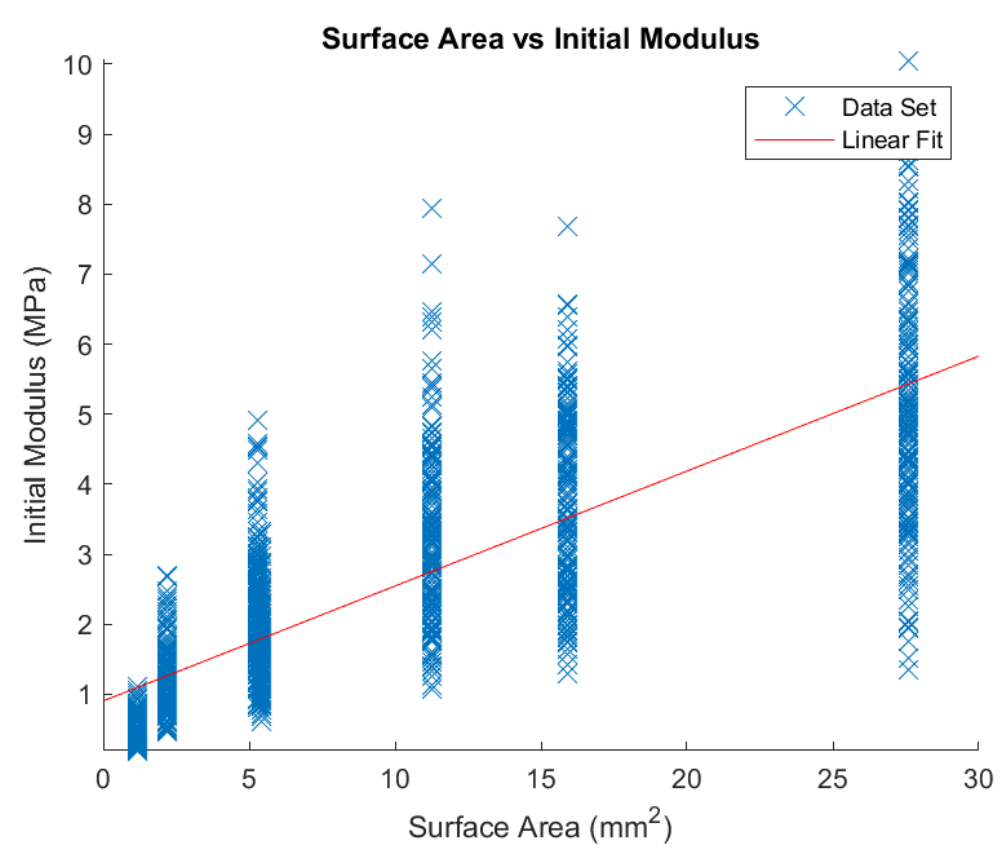

Figure 22: Calculated initial modulus plotted against indenter tip surface area; the red line shows the pairwise relationship from a simple regression. Changes in surface area were significant $(p<0.05)$ in affecting the initial modulus.

\subsubsection{Long-term Modulus Results}

The average long-term modulus of all the specimens is $1.97 \mathrm{MPa}$; the standard deviation was $1.42 \mathrm{MPa}(\mathrm{n}=1332)$. The linear fit of the change of the long-term modulus is given by Equation 11. Table 5 shows the calculated results for the long-term modulus and the results of the statistical analysis. This modulus is positive as the strain is taken to be negative in Equation 3. All three independent variables significantly affect the long-term modulus $(p<0.05)$. The high $\mathrm{R}^{2}$ value of 0.64 indicates that the variance of the results is well explained by the three variables. Beta values for speed and surface area are similar with surface area having a larger effect on the initial modulus than speed. The beta value for tension is an order of magnitude smaller that those of speed and surface area, indicating a weak effect compared to the other variables. Figures 23 , 24 and 25 show the calculated long-term modulus plotted against the three different independent 
variables.

$$
E_{\text {inf }}=0.020 * F_{\text {tension }}+38.45 * \text { Speed }+0.13 * A_{\text {surface }}-0.28
$$

Table 5: Step-wise regression results of the long-term modulus. Applied tension, indentation speed and indenter tip surface area were significant independent variables $(\mathbf{p}<0.05)$. The beta values of surface area and speed are an order of magnitude larger than tension and the beta value of surface area is larger than that of speed. This indicates surface area has the greatest effect on the long-term modulus followed by speed then tension. The $R^{2}$ value of 0.64 indicates that the independent variables explain the variance of the long-term modulus well.

\begin{tabular}{|l|l|l|l|l|l|}
\hline & Value & $\mathrm{p}$ & Lower 95\% confidence & Upper 95\% confidence & Beta \\
\hline Tension & 0.020 & 0.001 & 0.0082 & 0.032 & 0.055 \\
\hline Speed & 38.45 & 0.000 & 28.58 & 48.32 & 0.13 \\
\hline Surface Area & 0.13 & 0.000 & 0.12 & 0.13 & 0.79 \\
\hline Constant & -0.28 & & -0.52 & -0.033 & \\
\hline
\end{tabular}




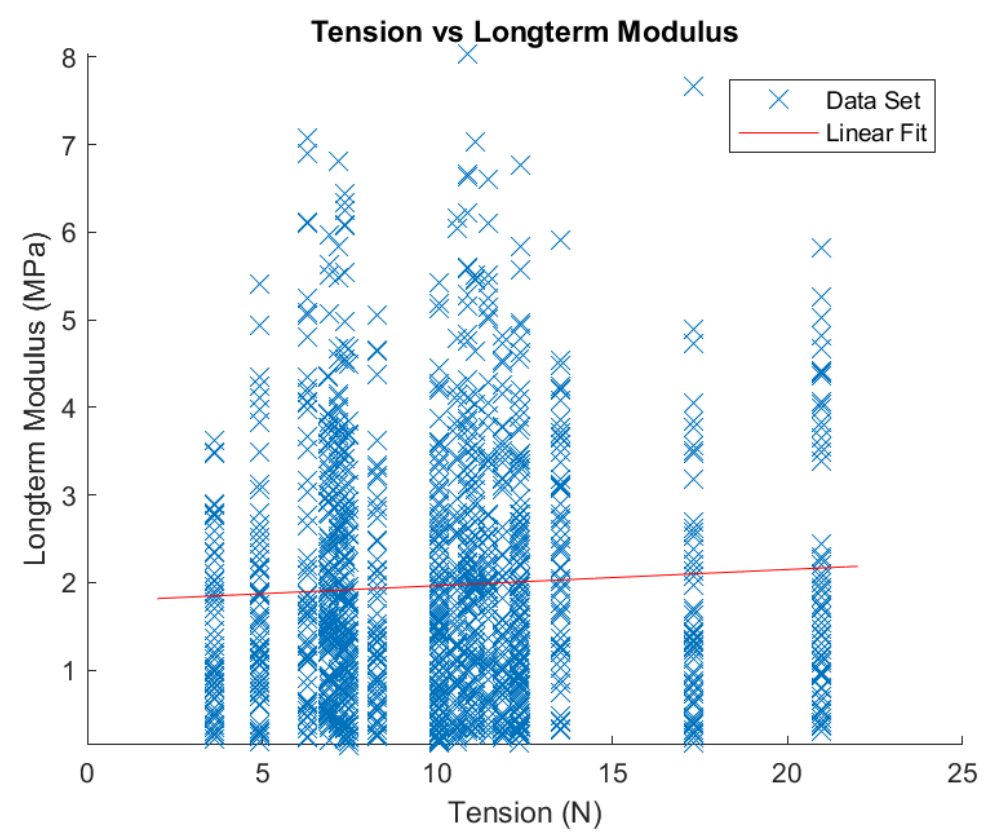

Figure 23: Calculated long-term modulus plotted against applied tension; the red line shows the pair-wise relationship from a simple regression. Changes in tension were significant $(p<0.05)$ in affecting the long-term modulus.

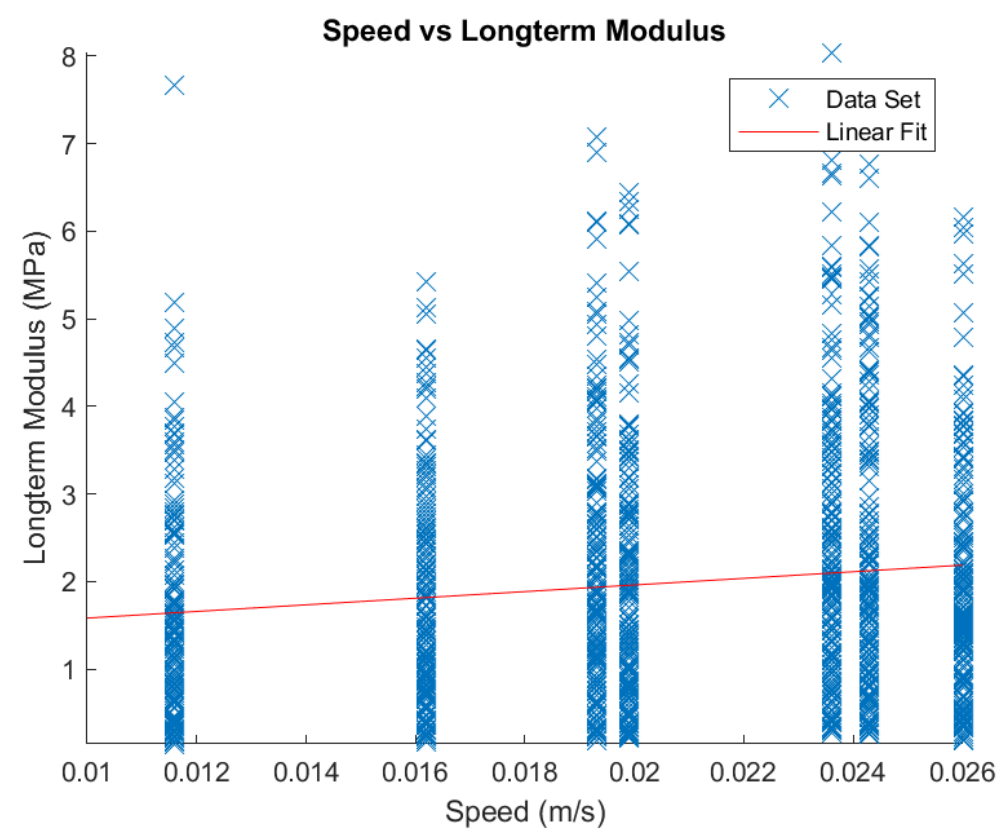

Figure 24: Calculated long-term modulus plotted against indentation speed; the red line shows the pair-wise relationship from a simple regression. Changes in speed were significant $(p<0.05)$ in affecting the long-term modulus. 


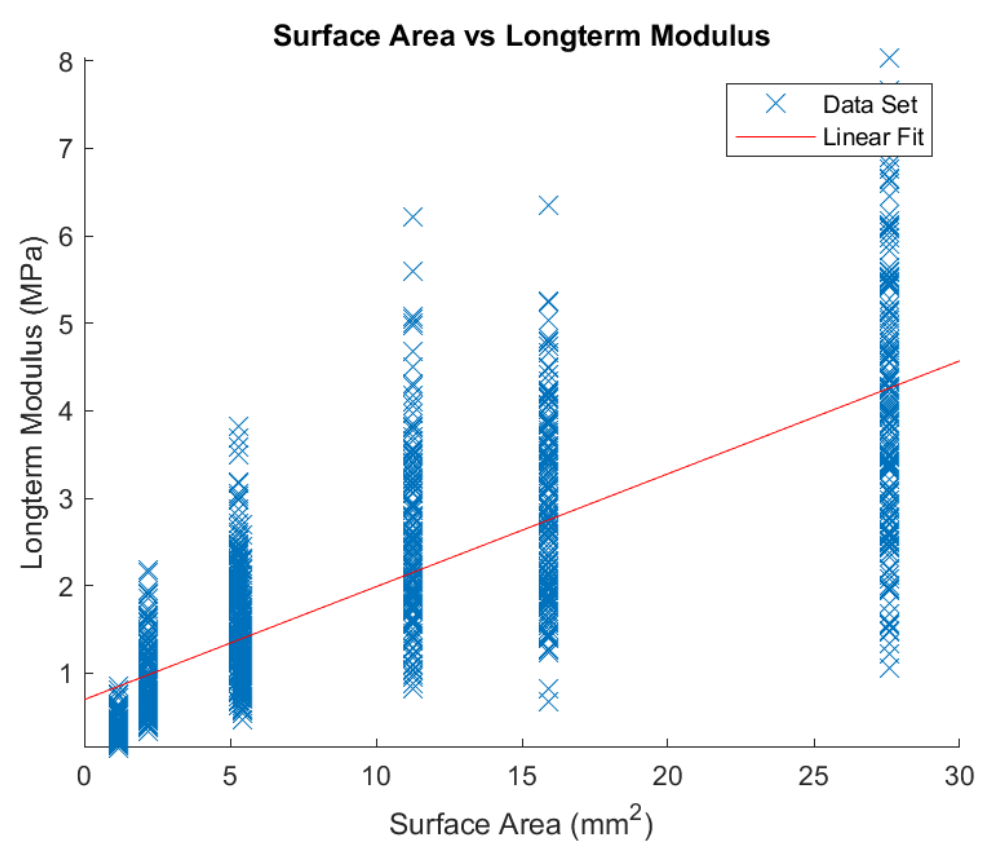

Figure 25: Calculated long-term modulus plotted against indenter tip surface area; the red line shows the pair-wise relationship from a simple regression. Changes in surface area were significant $(\mathbf{p}<0.05)$ in affecting the long-term modulus.

\subsubsection{First Relaxation Period Results}

The average of the first relaxation period of all the specimens is $0.21 \mathrm{~s}$; the standard deviation was $0.099 \mathrm{~s}(\mathrm{n}=1332)$. The change in first relaxation period is given by Equation 12 . Table 6 shows the calculated results for the first relaxation period and the results of the statistical analysis. All three independent variables significantly affect the first relaxation period $(\mathrm{p}<0.05)$. The low $\mathrm{R}^{2}$ value of 0.073 indicates that there is a weak relationship between changes in tension, speed and surface area and changes in first relaxation period. Beta values for tension and speed are similar however with opposite effects on the first relaxation period. The beta value for surface area is an order of magnitude smaller that those of tension and speed, indicating a weak effect compared to the other variables. Figures 26, 27 and 28 show the calculated first relaxation period plotted against the three different independent variables. 


$$
\tau_{1}=-0.025 * F_{\text {tension }}-5.14 * \text { Speed }+0.00087 * A_{\text {surface }}+0.33
$$

Table 6: Step-wise regression results of the first relaxation period. Applied skin tension, indentation speed and indenter tip surface area were significant independent variables $(\mathbf{p}<0.05)$. The beta value of tension and speed are an order of magnitude larger than surface area and approximately equal. This indicates that tension and speed have an equal effect on the first relaxation period and surface area has a lower effect. The $R^{2}$ value of 0.073 indicates that tension, speed and surface area have a weak relationship to the first relaxation period.

\begin{tabular}{|l|l|l|l|l|l|}
\hline & Value & $\mathrm{p}$ & Lower 95\% confidence & Upper 95\% confidence & Beta \\
\hline Tension & -0.0025 & 0.000 & -0.0038 & -0.0012 & -0.10 \\
\hline Speed & -5.14 & 0.000 & -6.25 & -4.04 & -0.24 \\
\hline Surface Area & 0.00087 & 0.004 & 0.00028 & 0.0015 & 0.076 \\
\hline Constant & 0.33 & & 0.30 & 0.36 & \\
\hline
\end{tabular}

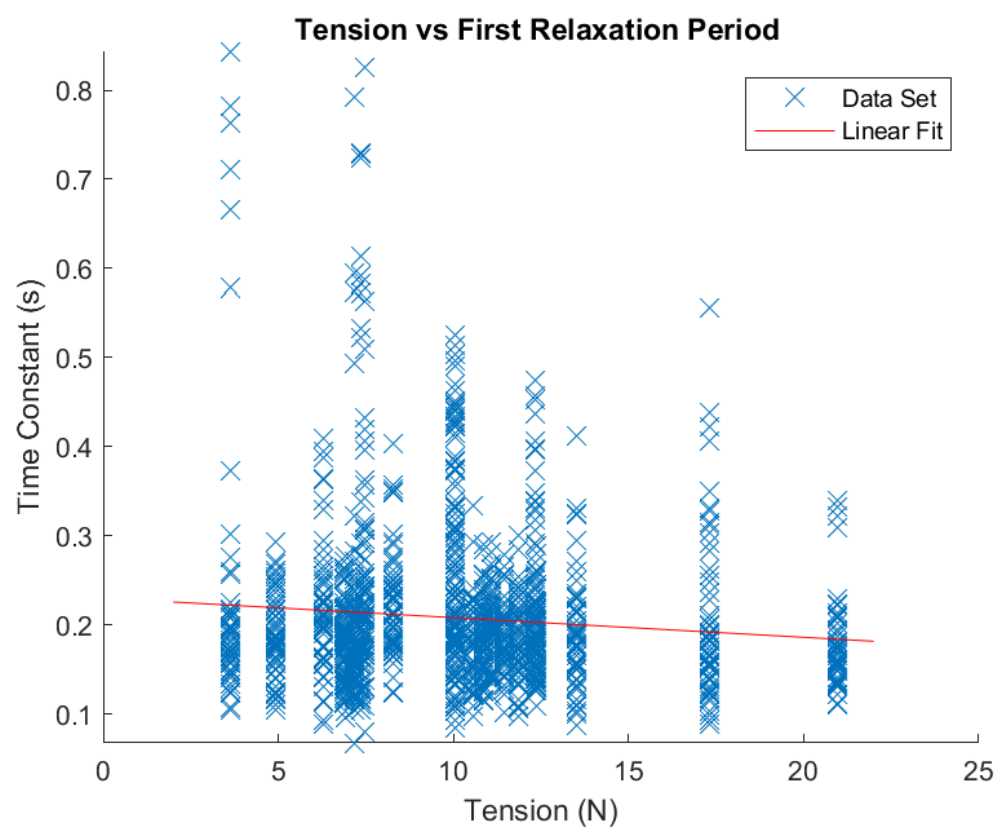

Figure 26: Calculated first relaxation period plotted against applied tension; the red line shows the pair-wise relationship from a simple regression. Changes in tension were significant $(p<0.05)$ in affecting the first relaxation period. 


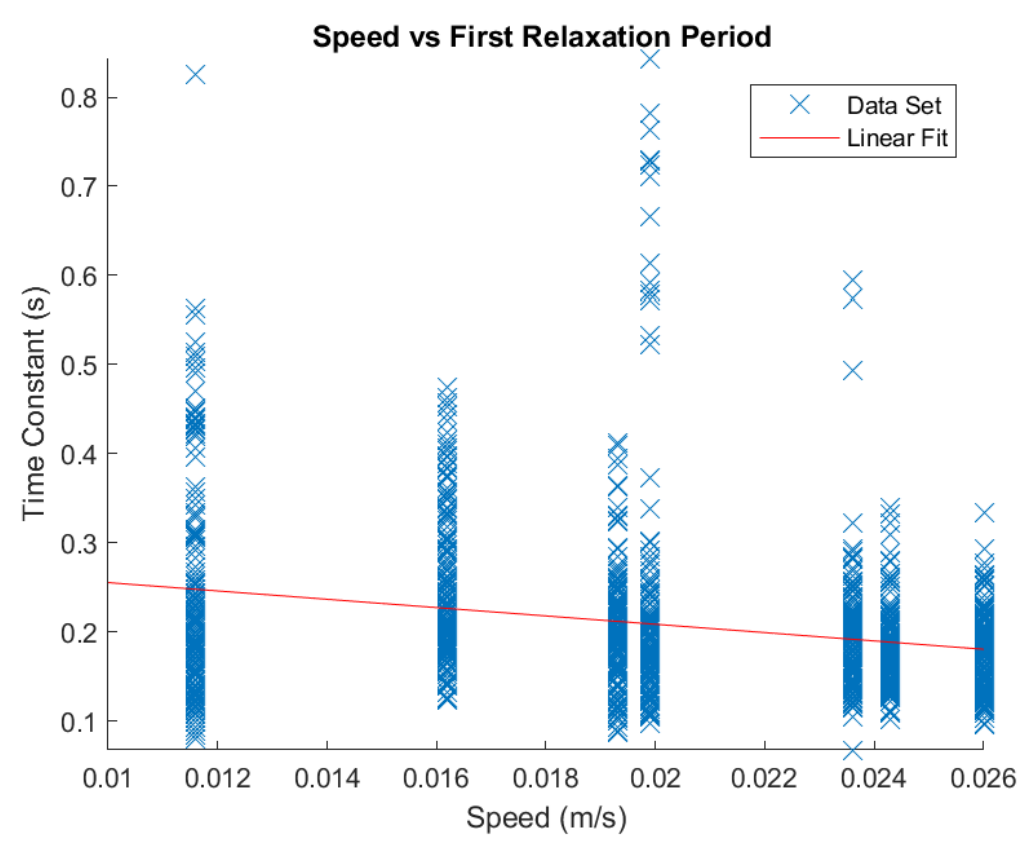

Figure 27: Calculated first relaxation period plotted against indentation speed; the red line shows the pairwise relationship from a simple regression. Changes in speed were significant $(p<0.05)$ in affecting the first relaxation period.

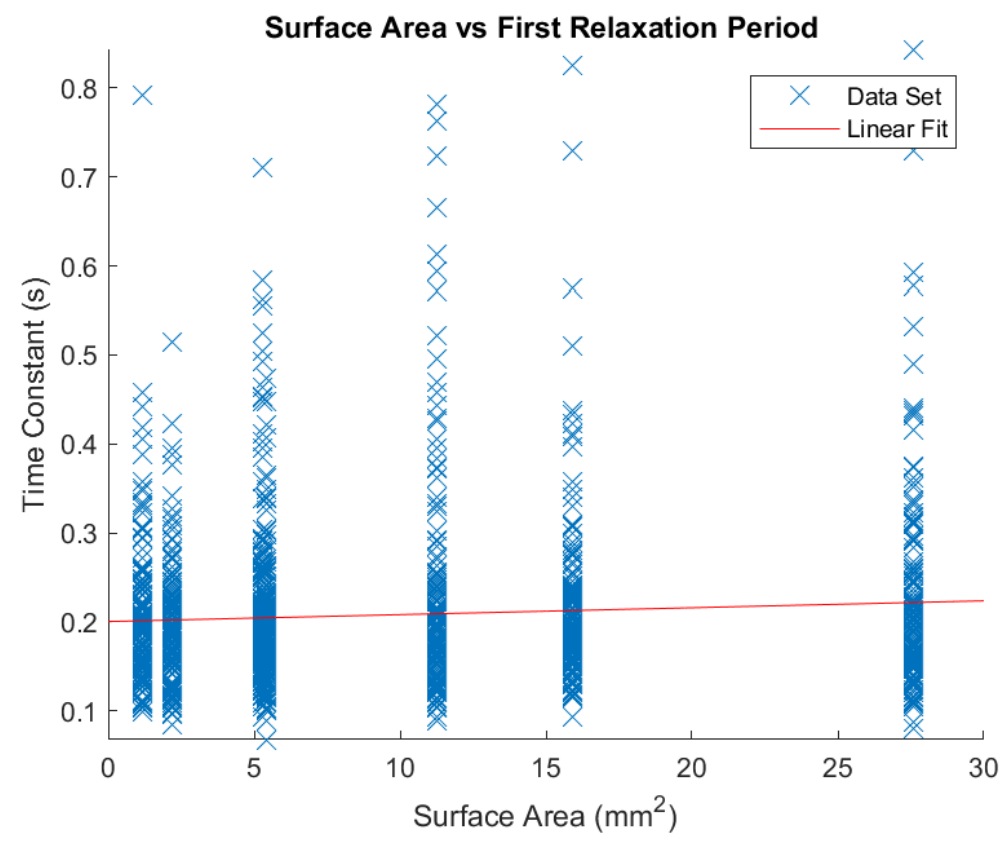

Figure 28: Calculated first relaxation period plotted against indenter tip surface area; the red line shows the pair-wise relationship from a simple regression. Changes in surface area were significant $(p<0.05)$ in affecting the first relaxation period. 


\subsubsection{Second Relaxation Period Results}

The average of the second relaxation period of all the specimens is $1.02 \mathrm{~s}$; the standard deviation was $0.31 \mathrm{~s}(\mathrm{n}=1332)$. The change in second relaxation period is given by Equation 13 . Table 7 shows the calculated results for the first relaxation period and the results of the statistical analysis. All three independent variables significantly affect the second relaxation period $(p<0.05)$. The low $R^{2}$ value of 0.073 indicates that there is a weak relationship between changes in tension, speed and surface area and changes in first relaxation period. Beta values for tension and speed are similar however with opposite effects on the first relaxation period. The beta value for surface area is an order of magnitude smaller that those of tension and speed, indicating a weak effect compared to the other variables. Figures 29, 30 and 31 show the calculated first relaxation period plotted against the three different independent variables.

$$
\tau_{2}=17.58 * \text { Speed }-0.0036 * A_{\text {surface }}+0.70
$$

Table 7: Step-wise regression results of the second relaxation period. Indentation speed and indenter tip surface area were significant independent variables $(\mathbf{p}<0.05)$ applied surface area was not significant and was omitted from the final regression equation. The beta value of speed and surface area are approximately equal indicating an equal effect on the second relaxation period. The $R^{2}$ value of 0.073 indicates that speed and surface area have a weak relationship to the second relaxation period.

\begin{tabular}{|l|l|l|l|l|l|}
\hline & Slope & $\mathrm{p}$ & Lower 95\% confidence & Upper 95\% confidence & Beta \\
\hline Tension & -0.0029 & 0.16 & -0.0069 & 0.0011 & \\
\hline Speed & 17.58 & 0.000 & 14.20 & 20.98 & 0.27 \\
\hline Surface Area & -0.0036 & 0.000 & -0.0054 & -0.0018 & -0.10 \\
\hline Constant & 0.70 & & 0.62 & 0.77 & \\
\hline
\end{tabular}




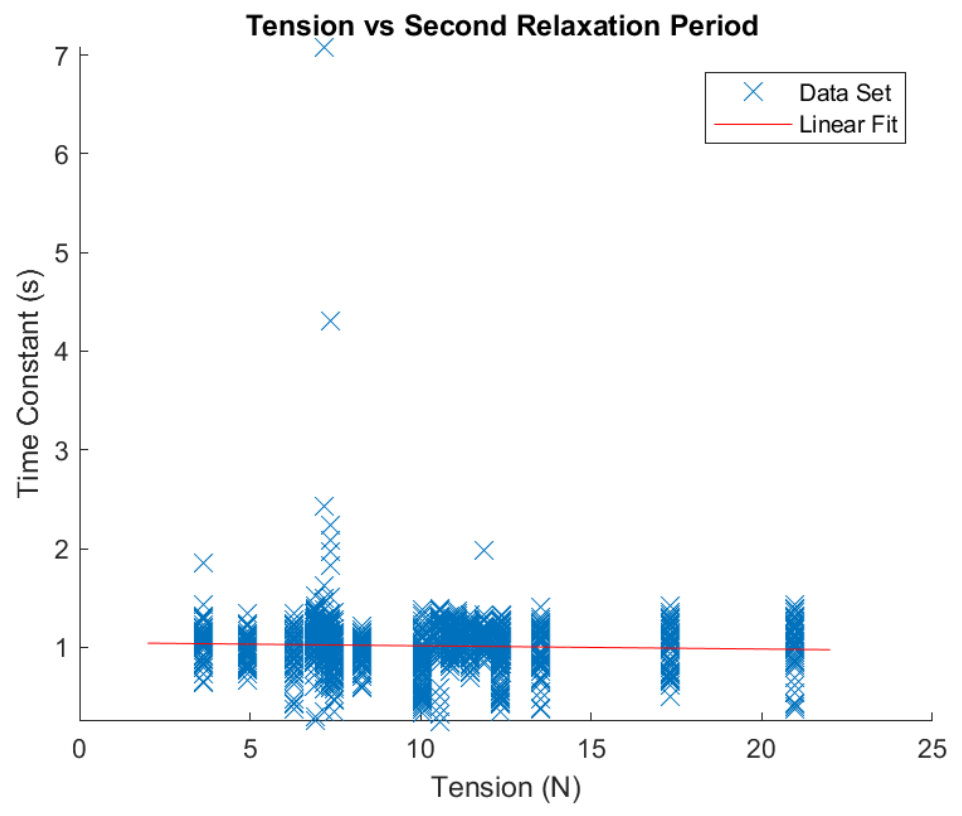

Figure 29: Calculated second relaxation period plotted against applied tension; the red line shows the pairwise relationship from a simple regression. Changes in tension were not found to be significant $(p=0.16)$ in affecting the second relaxation

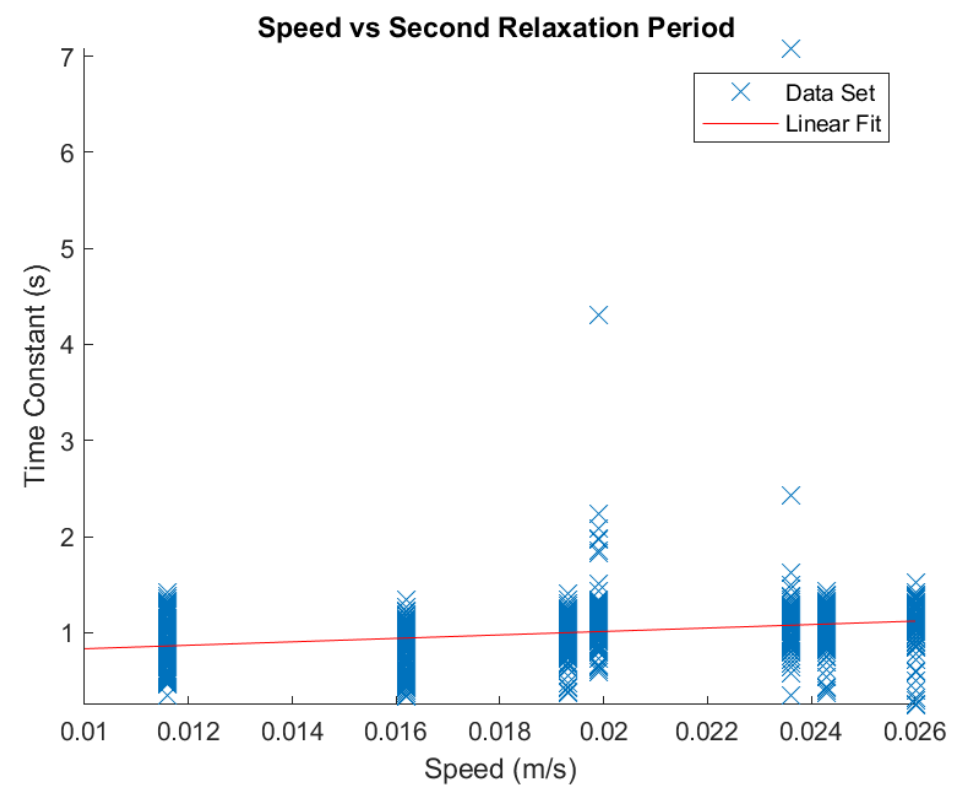

Figure 30: Calculated second relaxation period plotted against indentation speed; the red line shows the pairwise relationship from a simple regression. Changes in speed were significant $(p<0.05)$ in affecting the second relaxation period. 


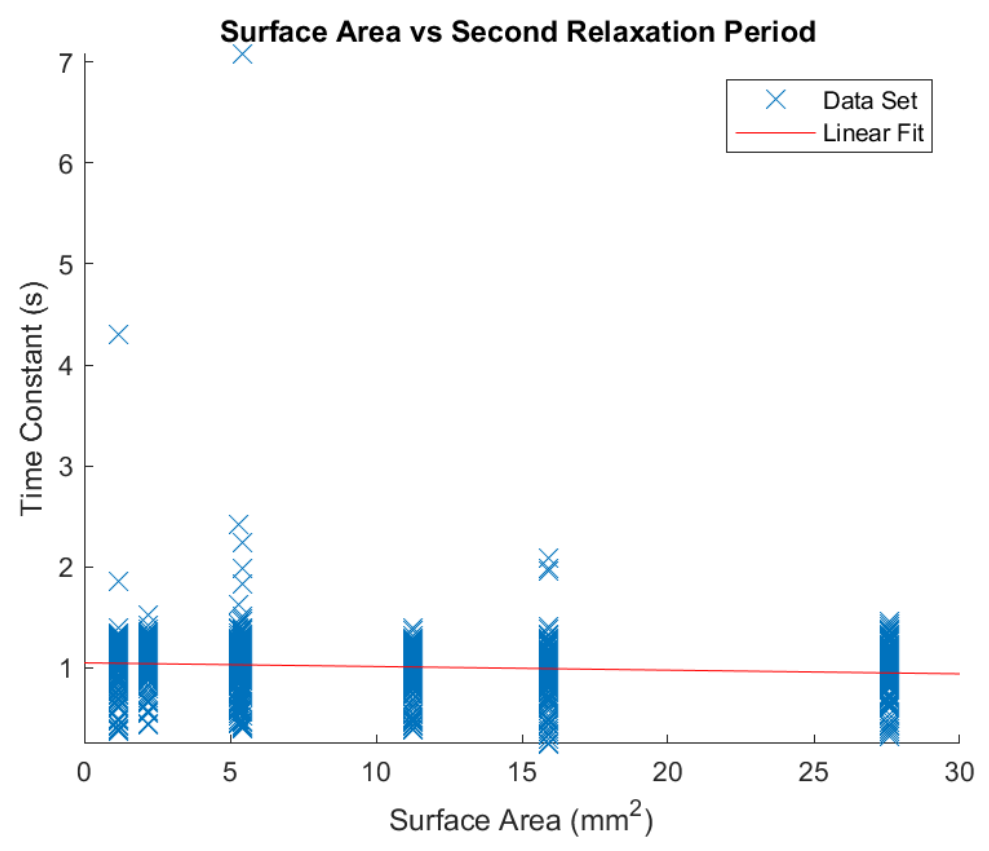

Figure 31: Calculated second relaxation period plotted against indenter tip surface area; the red line shows the pair-wise relationship from a simple regression. Changes in surface area were significant $(p<0.05)$ in affecting the second relaxation period.

\subsection{Discussion}

This study developed an analytical model for measuring the mechanical response to the indentation on skin tissue. Four material properties were derived from the experimental force profile. Indentation speed and tip surface area were found to affect the results while skin tension was found to negligibly affect the properties. 1332 separate data points were taken during the experiments and all data points are reported in the results sections. All combinations of tip geometries and indentation speeds were investigated with three separate tension settings allowing for the multivariate regression that was performed. A high standard deviation was observed for the 4 model parameters. As these parameters are necessarily positive due to their interpretation as physical moduli and relaxation periods assuming a normal distribution may not 
be appropriate. Outlier testing was investigated using a criterion of three standard deviations, this resulted in little change to the average parameter value and the calculated standard deviation. It can be inferred that the large amount of variance is due to the experimental conditions not as a result of outliers skewing the data.

The average results obtained in this experiment match reasonably well with those reported by Yazdi et al. As their experiment was the most similar to the one performed here, it gives confidence that the trends extracted from the experimental data are equally valid. No other literature investigating the change in indentation or tensile properties of tissue has been found by the author. Therefore, the trends presented here cannot be directly compared to other works.

The maximum force results are difficult to compare to others in literature due to the differences in experimental procedure. Isaza and Ramirez obtained force values of approximately $1 \mathrm{~N}$ for a spherical indenter indenting $5 \mathrm{~mm}$ [39]. Tran et al. and Then et al. found similar maximum forces [30], [35]. Yazdi et al. recorded forces of approximately $0.02 \mathrm{~N}$ for their in vivo experiments of human skin, their indentations were only $0.5 \mathrm{~mm}$ deep [46]. Dai et al. and Jachowicz et al. obtained similar values to Yazdi [41], [43]. Without a more complete break down of the geometry used in these experiments it is difficult to estimate if the fitting equation used here would predict the results found in these experiments.

Papers by Lui et al. and Yazdi et al. were the only literature found which derived the mechanical response in a similar fashion to this paper [45], [46]. Lui et al. performed a finite element model and tested a chemical solution so their results are not applicable to this analysis. Yazdi et al. performed their experiments in vivo on human thighs. They reported an average initial modulus of 7.21 MPa an average long-term modulus of 4.74 MPa for their experiments [46]. The longterm modulus has been interpreted in the model derivation to be approximately equal to the 
quasi-static elastic modulus. Other experiments using human tissue reported Young's moduli values close to 100 times smaller than reported here and by Yazdi et al. Pailler-Mattei et al. reported a value of $35 \mathrm{kPa}$ for the Young's modulus of human skin [38]. Chen et al. reported similar values of modulus [42]. The difference in deriving the moduli values and experimental set up might account for some of that difference. Ni Annaidh et al. compiled some results from difference sources on the reported Young's Modulus for human skin [9]. It showed up to two order of magnitude difference in reported results experiment to experiment.

Yazdi et al. reported a result of $0.38 \mathrm{~s}$ for their first relaxation period [46]. Damping coefficients have been reported by Sandford et al. and Parker et al. [32], [72]. Their derivation of a damping coefficient corresponds approximately to the interpretation of the first relaxation period. Sandford et al. reported a damping coefficient of approximately 0.6 with in vivo human tissue. Parker et al. reported their damping coefficient as a function of the applied strain and force with their damping values varying between 2 and $15 \mathrm{~N} \cdot \mathrm{s} / \mathrm{m}$. Yazdi et al. reported a result of $5.01 \mathrm{~s}$ for their second relaxation period [46]. Shen et al. reported a fast relaxation time of $7 \mathrm{~s}$ for individual collagen fibers [11]. The second relaxation period has been interpreted here to be related to the relaxation of the collagen matrix.

The initial assumption of a viscoelastic material aids in the interpretation of the physical meaning of the extracted parameters. As stated, the two moduli are representative of the initial and long-term response. The long-term modulus is the elastic modulus at equilibrium of the tissue material. As the indenter deforms the skin surface the fluids in the tissue push against the indenter; the amount of force exerted by the interstitial fluids is rate dependant. Using this model as a base for the skin mechanics the initial modulus is the superposition of the long-term elastic modulus and this fluid response to the applied strain. The first relaxation period is attributed to 
the rate of dissipation of any fluid pressure gradients within the tissue structure. The second relaxation period is attributed to the relaxation and equilibrium rate of the collagen fibers.

Biological sample variability is a major hurdle in determining if the effect observed is a result of the independent variable or the material itself. Previous studies and experiments have reported large between sample variability. The sample variability of presented results in Ni Annaidh is 15 to 80 percent [9], Lagan is 10 to $40 \%$ [21]. Similar ranges are seen in work by Ottenio [10], Sandford [32], Mayrovitz [34], and others. Without an accurate measure, or even estimate, of the amount of sample variability it is difficult to ascertain what is sample variability and what is a trend in the data. While care was taken to ensure that the samples were as similar as possible from one test to the next, there was considerable variability within the samples. Samples might have a thicker or thinner fatty layer. Occasionally a muscle layer would be present immediately below the skin. Obviously, these observed structural differences have the possibility of significantly changing the observed response.

There is a variance of approximately $35 \%$ not explained by the linear regression equation for the two derived moduli terms. This suggests an upper limit of the amount of biological variability that could be assumed. Of course, there are other possible sources of error that could contribute to this variability. If the assumption of $35 \%$ inherent sample variability is accepted then the results of maximum force, first and second relaxation periods still exhibit substantial unexplained variance. The $\mathrm{R}^{2}$ values of less than 0.1 for these three linear fits shows a very weak correlation between the results and changes in the three independent variables. The impact of the assumed sample variability is much more significant than the variables themselves.

The low correlation of the force and relaxation period results also indicates that some other factor than the three variables investigated is responsible for the variance in the results. The 
results for the initial and long-term modulus are far more correlated to the linear model. This would suggest that these properties are more dependent on the three variables investigated than the force and relaxation period results.

The experimental data suggest that the skin tension does not have much effect on material properties. The tension is not statistically significant in the experimental results for maximum force and second relaxation period. While the tension is statistically significant in the results for the two moduli the beta value of tensions in both these results are an order of magnitude below the other variables. In the results for the first relaxation period, the tension is both significant and of equal weight to the other variable the linear regression model. This linear regression fit has the lowest $\mathrm{R}^{2}$ value and the changes in the results is possibly overshadowed by sample variability.

This result is interesting; given that skin tissue is made up of collagen and elastin fibers, preloading the skin sample should increase the force response to a fixed indentation.

Conceptually, preloading the skin would orient and stretch the collagen fibers before the induced strain. This should result in more fibers being initially activated and more fiber remaining activated at equilibrium. Skin is in a state of natural tension in vivo; however, the magnitude of this tension is not documented. It is possible that the applied strain field is not applied uniformly through the experimental samples. The sample preparation and handling process may also have resulted in tissue degradation or destroys the collagen links within the sample. During the experiment, after approximately 10 minutes, the recorded tension had little change from the start of testing to the end. It is possible that the collagen fibers may have completely relaxed in the sample despite a tensile force being recorded. In vivo testing would be required to compare the results obtained in this experiment to the live model to fully explore the impact that skin tension has on the response to an indentation force. 
Skin tissue is thought to be a viscoelastic material, it is inherently assumed that the strain rate has a significant impact on the measured force and therefore the measured physical properties. The experimental results suggest that speed is indeed a significant influence on all five experimental measures. The experimental results agree with the expected results from the model. The maximum force and initial modulus increase with increasing speed, as the strain rate increases there should be higher pressure gradients within the tissue which would contribute to a larger reaction force which is converted into the initial modulus. A decreasing first relaxation period with increasing speed makes sense from two perspectives. First, the relative increase in initial modulus to long-term modulus is not equal, the initial modulus increases at a faster rate than the long-term modulus. Mathematically this requires a steeper response (a larger exponent) to reach the long-term modulus within the relaxation time. Physically the higher strain rate creates a larger pressure gradient which will need to dissipate.

Since the experimental results suggests that skin tension does not impact the material properties, the long-term modulus should remain constant (within sample variability). Once the sample has reached equilibrium the elastic modulus should be independent of the rate at which the strain was applied or the geometry of the indenter. Observed results do not support this theory; as tip speed and surface area have an influence on the long-term modulus. The long-term modulus increasing as the tip speed increases is an interesting result, as there does not appear to be a physical mechanism to explain the increase. If the indentation area has reached an equilibrium, there should be no more interstitial fluid movement and the reaction force should simply be the elastic compression. A negative correlation between speed and long-term modulus would make more sense. As the strain rate increases the interstitial pressure increases which could damage the collagen fiber matrix. The loss of collagen fiber integrity could result in more 
interstitial fluid leaving the strain area resulting in a decrease of the long-term modulus. The surface area has the largest effect on the change in long-term modulus. This result is also interesting as it should also not occur. This is likely a mathematical artifact of the definitions of the strain affected zone. The strain affected zone was assumed to be a fixed ratio of the indentation depth, the ratio is also likely affected by other variables. The simplification to a onedimensional stress state may also contribute to the apparent change in modulus values. A true material modulus should be independent of the method of indentation.

The increase in the second relaxation period with increasing velocity is equally interesting. While Chen et al. showed viscoelastic properties of individual collagen fibers, they did not show any changes in the relaxation periods of the fibers [11]. Their experiments used a low applied strain rate and did not vary it. Without direct experimental results, conceptually, the relaxation rate should be independent of strain rate. The positive slope indicates that the collagen fibers are taking longer to relax as strain rate increases. This result would make more sense as a function of surface area. Engaging more collagen fibers making the system, as a whole, slower to relax. The results do not support that scenario as the surface area has a negative slope indicating the system relaxes faster.

The increase in initial and long-term moduli with increase in the tip surface area is a counter-intuitive result. Both the conical and cylindrical tips show an increase in modulus at also equal slopes. It would be expected that smaller surface areas, especially the conical tipped indenters, would show an increase in modulus due to stress concentrations around the tip. In the cylindrical tipped indenters, the strain affected zone was a function of the tip radius. This was not the case with the conical tipped indenters which had a fixed strain zone. The strain affected zone does not seem to affect the result. This makes sense, since the collagen fibers are a finite length 
much smaller than the zone itself. When engaging a larger volume with the cylindrical tips there is no change to the relative contribution of each individual fiber. Based on the geometry of the assumed strain affected surface there should be no change with the cylindrical tips. The surface slope is independent of the strain affected area. This is not true for the conical tips in which the surface is affected by the cone angle.

The definition of the strain zone was assumed to be solely a function of the indenter tip type and geometry; this assumption is likely not valid. The affected radius due to indentation has not been investigated as the predictive models used in the analysis of the mechanical properties has not required it. The model proposed in this investigation is dependant on a definition of stress and strain affected zone. It is possible that this is another independent variable that could impact the mechanical properties; however, it is more likely that the strain affected zone is a function of the same variables as the indentation response.

The results of this study demonstrate that the surface area and geometry of the indenter tip plays a significant role in the measured moduli. The applied strain rate has only a minor effect on the moduli and relaxation periods. The applied range of geometry and strain rate is admittedly limited; however, given the unexpected results warrant further investigation. In vivo experiments would also be essential to validate the findings of this study.

\subsubsection{Conclusion}

The experimental results support several key assumptions about the mechanical properties of skin tissue. The indenter speed has a significant effect on the response properties, confirming the primary assumption of skin tissue as a viscoelastic material. Indenter tip geometry also significantly affected the response properties. It was found that the skin tension of 
the skin had a negligible effect on any mechanical properties. This is a surprising and interesting result due to the assumptions made about how tissue responds to the indentation force.

Two notable and counter intuitive results observed was the increase in long-term modulus as a function of tip surface area and indenter speed, and the poor correlation to the linear regression model of the maximum force, first and second relaxation periods. The long-term modulus should be a material function independent of how strain is applied. The poor correlation suggests other factors have a more significant effect on some of the response properties than the variables used in the experiments. 


\section{Chapter 3: Effect of Skin Tension, Needle Diameter and Insertion Velocity on the Fracture Properties of Porcine Tissue}

\subsection{Differences from the Journal Submission Version}

The introduction section of the journal submission version has been omitted from the thesis. The relevant theory has been developed in Sections 1.1 through 1.3 and Sections 1.8 through 1.10. The mathematical model has been kept in a condensed version for reference in Section 3.2. Numbering of equations has reset; numbering of figures and tables has not.

\subsection{Model Development:}

The work required to fracture tissue is a combination of the work required to propagate the line crack through the tissue plus the work required to deform this tissue to accommodate the needle [63], [65]. Occasionally, the work contribution of friction or tissue cutting is included [48], [58]. The major contribution is typically assumed to be the work required to fracture the skin, with the work of deformation being the next contributor.

The scenario of an object penetrating an infinite plate is one that is not handled well by classical fracture mechanics. Conceptually, this scenario is a type I fracture, but the plane stress has been replaced with a point force at the center of the crack. Michell derived some equations for relating a point force to the perpendicular stresses in an elastic solid [69]. These equations were derived without taking into account stress concentrations caused by a fracture or the local regions of zero stress around the crack perimeter. The equation for the fracture work is given 
below in Equation 1; where $J_{I C}$ is the fracture toughness of the tissue, $\delta A$ is the change in crack area (along the direction of the needle trajectory).

$$
\delta W_{\text {fracture }}=J_{I C} \delta A
$$

It is commonly assumed that tissue is incompressible which would result in the deformation work being zero. This assumption is valid for certain analyses but does not hold for the deformation of the skin to accommodate the needle shaft. The applied strain is not quasistatic; therefore, viscoelastic resistance will need to be overcome contributing to the net work of the system. This can be equated to an elastic deformation; resulting in strain energy associated with the widening of the crack. If it is assumed that this strain is purely elastic, the work required to deform the skin is given by the volumetric integral in Equation 2. The stresses and strains are in the plane perpendicular to the crack path.

$$
\delta U=W_{\text {elastic }}=\int_{V o l} \sigma_{i j} \epsilon_{i j}
$$

The Cartesian field stress from Michell is given in Equation 3 [69]; where $F$ is the applied load, $\theta$ is the radial angle, $\lambda$ and $\mu$ are the first and second Lamé material parameters and $r$ is the radial distance from the center.

$$
\sigma_{x}=\sigma_{y}=\frac{\mu}{\lambda+2 \mu} \frac{F}{4 \pi} \frac{\cos \theta}{r^{2}}
$$

It is apparent as the radius approaches zero the stress becomes infinite which is impossible. The equation is equally meaningless for large values of radius. A defined zone of stress is required to make Equation 2 meaningful. Similarly, a defined strain zone is required to compute Equation 2.

Shergold and Fleck derived a method for calculating the strain energy for this scenario 
using an Ogden model [66]. However, this solution needs to be calculated analytically. Further it requires some assumptions about the size of the crack, denoted as a, versus the radius of the needle, denoted as $\mathrm{R}$. For low values of a/R the strain energy dominates the fracture work, however at higher values $(\mathrm{a} / \mathrm{R} \approx 4)$ the energy required for crack propagation is the dominant term. Based on the pictures provided by Shergold and Fleck [65]; it appears that the fracture is only as large as required to accommodate the punch.

It would be simpler and convenient to define a zone in which the strains and stresses are applied. If it is assumed that the only contributors to the net work are the fracture and the elastic deformation the system work becomes Equation 4.

$$
\delta W_{\text {sys }}=\delta W_{\text {fracture }}+\delta W_{\text {elastic }}
$$

If it is then assumed that the stress and strain affected volume is equal and constant, and the stress and strain fields are radial; Equation 2 becomes Equation 5.

$$
W_{\text {elastic }}=\sigma_{r} \epsilon_{r} V
$$

Since the assumption that the stress is radially constant; the angular component of Equation 3 is removed and can be rewritten as Equation 6.

$$
\sigma_{r}=\frac{\mu}{\lambda+2 \mu} \frac{F}{4 \pi r^{2}}
$$

It will then be assumed the radius of the affected zone is not large, and it can be assumed that the interface stress of the needle and skin $\left(r=r_{\text {needle }}\right)$ is constant through the zone.

The definition of plane strain is Equation 7. The skin surface will deform from the closed crack to an annulus of the same radius as the needle to allow the needle to pass.

$$
\epsilon=\frac{\Delta A}{A}=\frac{\pi r_{\text {needle }}^{2}}{A}
$$

Since it has been assumed that the volume affected by the strain is equal to the elastic 
volume, the volume in Equation 5 can be rewritten as a function of that area and the crack depth. Substituting Equations 6 and 7 into Equation 5 results in Equation 8, where $\Delta A=\pi r_{\text {needle }}^{2}$.

$$
W_{\text {elastic }}=F_{\text {elastic }} \delta z=\frac{\mu}{\lambda+2 \mu} \frac{F_{\text {total }}}{4 \pi r_{\text {needle }}^{2}} \frac{\Delta A}{A} A \delta z
$$

This result eliminates the need to define the planar area of the affected zone, further it now also becomes independent of the thickness of the specimen and the radius of the needle. The reaction force resulting from the elastic deformation of the crack is Equation 9.

$$
F_{\text {elastic }}=\frac{\mu}{\lambda+2 \mu} \frac{F_{\text {total }}}{4}
$$

The Lamé material parameters, $\lambda$ and $\mu$ are given by Equations 10 and 11 , where $\mathrm{E}$ is the Young's Modulus and $v$ is the Poisson's ratio.

$$
\begin{gathered}
\mu=\frac{E}{2(1+v)} \\
\lambda=\frac{E v}{(1+v)(1-2 v)}
\end{gathered}
$$

Equation 12 gives the factored first term of Equation 9.

$$
\frac{\mu}{\lambda+2 \mu}=\frac{1}{2\left(\frac{v}{1-2 v}+1\right)}
$$

The reaction force of the crack propagation can also be made independent of the thickness as shown in Equations 13 and 14. Where $a$ is the crack length, which will be assumed to equal the diameter of the needle.

$$
\begin{gathered}
\delta W_{\text {fracture }}=F_{\text {fracture }} \delta z=J_{I C} \delta z a \\
F_{\text {fracture }}=J_{I C} a
\end{gathered}
$$

Combining Equations 9 and Equation 14 into Equation 4 and rewriting into force terms 
results in Equation 15.

$$
F_{\text {total }}=J_{I C} a+\frac{\mu}{\lambda+2 \mu} \frac{F_{\text {total }}}{4}
$$

For an incompressible material $(v=0.5)$ the lambda term in Equation 11 becomes infinite. Other literature has given values of 0.36 [40] and 0.45 [48]. For a Poisson's ratio of 0.36 gives a value of approximately 1/4.5 for Equation 12 and a Poisson's ratio of 0.45 gives a value of $1 / 11$. Since skin tissue is commonly assumed to be incompressible a Poisson's ratio value close to 0.5 is more appropriate. However, even assuming a ratio value of 0.36 , Equation 9 reduces to $F_{-}$elastic $=F_{-}$total/18. Since this value will be small compared to the value of the total force this term will be neglected. The fracture toughness is then given by Equation 16 .

$$
J_{I C}=\frac{F_{\text {total }}}{a}
$$

\subsection{Methods and Materials}

Skin fracture testing was performed using fresh porcine belly skin sourced from a local abattoir. The sample was subdivided into squares approximately $175 \mathrm{~mm}$ by $175 \mathrm{~mm}$, these were then trimmed to a thickness of approximately $10 \mathrm{~mm}$ to $12 \mathrm{~mm}$ with emphasis on ensuring constant thickness and removing the muscle tissue where possible. The sample was then sutured and mounted to brackets that, when tightened, provided tension in the in-plane directions, an SType load cell (Interface Inc. SM-25, Scottsdale, Arizona) was mounted along each axis measuring the applied tension. Medical ballistics gel (Humimic Medical \#0 Medical Gel, Greenville, South Carolina) was placed underneath the sample to simulate a muscle layer. Figure 32 shows the experimental set up. 


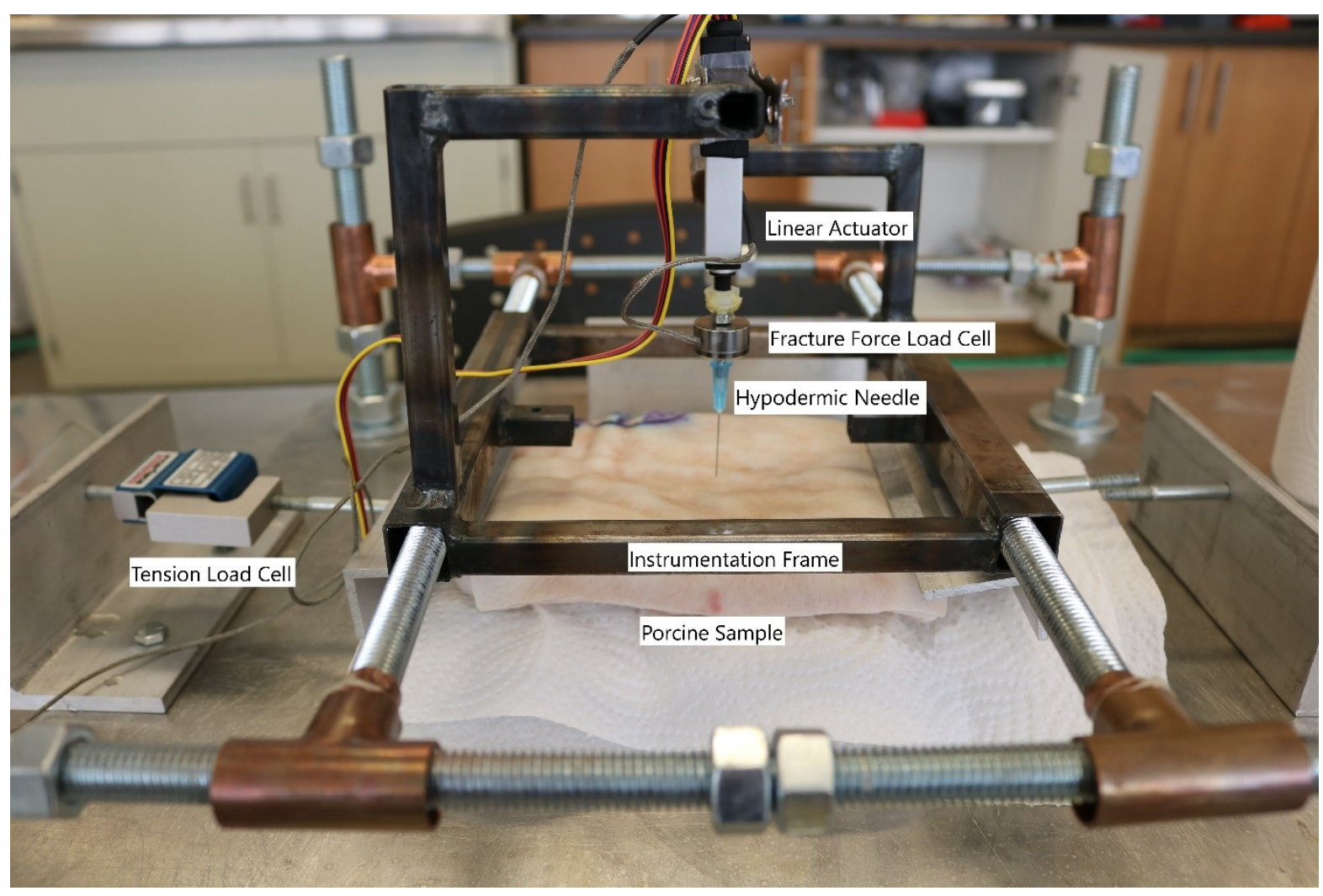

Figure 32: Experimental apparatus

A geared drive train linear actuator (Actuonix Motion Devices Inc. L12-P Micro Saanichton, British Columbia) controlled using Actuonix's linear control board was used to extend the indenter tips to the skin surface. For testing seven speeds settings were chosen and calibrated using the on-board variable resistor. The speeds were $0.0116 \mathrm{~m} / \mathrm{s}, 0.0162 \mathrm{~m} / \mathrm{s}, 0.0193$ $\mathrm{m} / \mathrm{s}, 0.0199 \mathrm{~m} / \mathrm{s}, 0.0236 \mathrm{~m} / \mathrm{s}, 0.0243 \mathrm{~m} / \mathrm{s}$ and $0.0260 \mathrm{~m} / \mathrm{s}$.

Five sizes of hypodermic needles were used: $0.4 \mathrm{~mm}, 0.5 \mathrm{~mm}, 0.8 \mathrm{~mm}, 0.9 \mathrm{~mm}$ and 1.2 $\mathrm{mm}$ diameter. The $0.4 \mathrm{~mm}$ and $0.5 \mathrm{~mm}$ needles were $20 \mathrm{~mm}$ in length and the others were 35 $\mathrm{mm}$ in length. These needles were attached to an inline load cell (Omega Engineering Inc. LCMFL-20N, Stamford, Connecticut) which was attached to the actuator.

The prepared pork samples were wrapped in paper towel, soaked in water, and refrigerated for a minimum of 2 hours. The samples were then brought up to room temperature 
for approximately 1 hour, still wrapped in the paper towel and then sutured into the clamps. The sample was tensioned in the transverse plane and allowed to relax for approximately 10 minutes before testing commenced. It was found that after 10 minutes the change in recorded tension values for the test duration were minimal. Fracture tests were done on 12 sites arranged in a 4 by 3 grid with a minimum of 60 millimeters from the edge of sample. All testing locations were marked. Calibration testing showed no change in test data gathered at the beginning versus the end of the test period. Calibration also found no change in recordings with test points a minimum of $5 \mathrm{~mm}$ apart. Data collection and linear actuator control was done with an Arduino Due system connected to the Actuonix Linear Actuator control Board. The needle was set to travel to a displacement of approximately 6 millimeters below the surface of the sample ensuring full penetration. Total time from beginning of suturing to end of testing was approximately 1 hour; care was taken to ensure that the pork sample did not excessively dry during testing.

Three samples were used over the seven speed testing cycles at a constant needle diameter. Once complete a new batch of three samples were prepared and a different sized needle was tested.

Statistical analysis was done using Stata SE 15.1 (StataCorp LLC, College Station, Texas). The effect of skin tension, insertion speed and needle diameter on the maximum force and the fracture coefficient was evaluated using step-wise linear regression. An independent variable was removed if its effect was not significant and the regression was repeated until no more variables were removed. The strength of the effects of independent variables on the dependent variable were compared with beta, the standardized regression coefficient; beta measures the change in the dependent variable due to a change in the independent variable, in multiples of the standard deviations of the respective variables. Unlike the regression coefficient, 
beta allows comparison of variables of different units and with different orders of magnitude. The higher the beta value, the greater the effect the independent variable has on the dependent variable. Beta values are not reported for insignificant variables. The overall strength of the regression was also assessed with the coefficient of determination $\left(\mathrm{R}^{2}\right)$.

\subsection{Results}

Maximum recorded force and calculated fracture coefficient results are presented in the following sections. Figure 33 shows an example of the recorded force data, the data output was manipulated so the maximum recorded force would correspond to time 0 . Maximum force is presented so that these experimental results may be compared to those in literature.

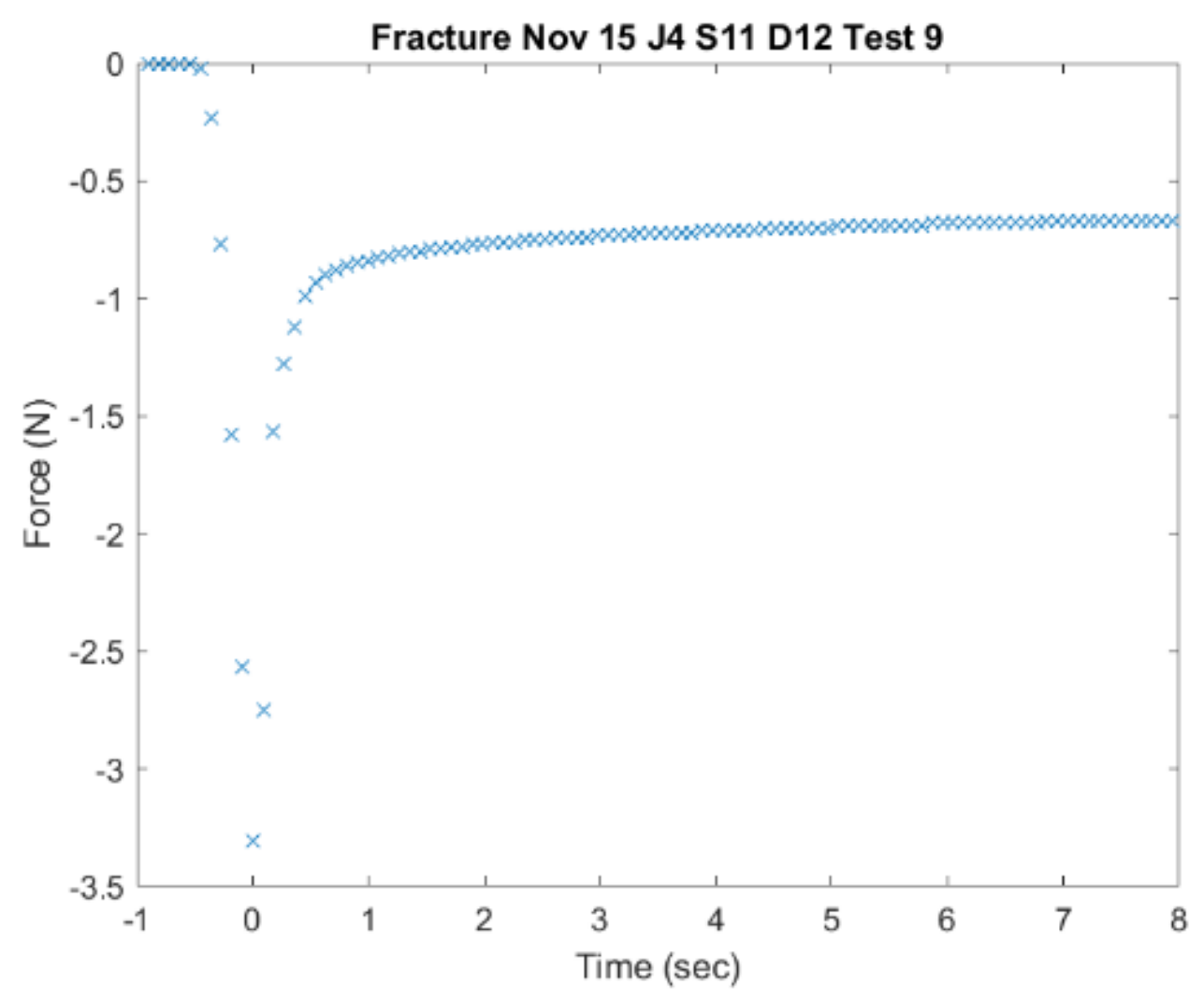

Figure 33: Recorded fracture force trial example 


\subsubsection{Force Results}

The average maximum force recorded of all specimens was $-1.30 \mathrm{~N}$; the standard deviation was $0.88 \mathrm{~N}(\mathrm{n}=420)$. The equation of the linear regression is given by Equation 13 . The $\mathrm{R}^{2}$ value of this fit was 0.12 indicating that variation in the results was poorly explained by the linear regression. Speed was found to approach statistically significance $(\mathrm{p}<0.05)$, however was neglected in Equation 13. Table 8 details the results of the statistical analysis. Figures 34, 35 and 36 show the maximum force plotted against each individual variable.

$$
F_{\text {max }}=-0.069 * F_{\text {tension }}-1.39 * D_{\text {needle }}+0.75
$$

Table 8: Step-wise regression results of the maximum force. Insertion speed approached significance as an explanatory variable but was omitted from the final regression equation. Applied skin tension and needle diameter were significant independent variables $(p<0.05)$. The similar beta values indicate that both tension and diameter have an approximately equal effect on the maximum force. The $R^{2}$ value of 0.12 indicates that tension and diameter have a weak relationship to maximum force.

\begin{tabular}{|l|l|l|l|l|l|}
\hline Variable & Coefficient & $\mathrm{p}$ value & Lower 95\% confidence & Upper 95\% confidence & Beta \\
\hline Tension & -0.069 & 0.000 & -0.091 & -0.046 & -0.31 \\
\hline Speed & -17.91 & 0.056 & -36.29 & 0.48 & \\
\hline Diameter & -1.39 & 0.000 & -1.81 & -0.97 & -0.34 \\
\hline Constant & 0.75 & & 0.50 & 2.05 & \\
\hline
\end{tabular}




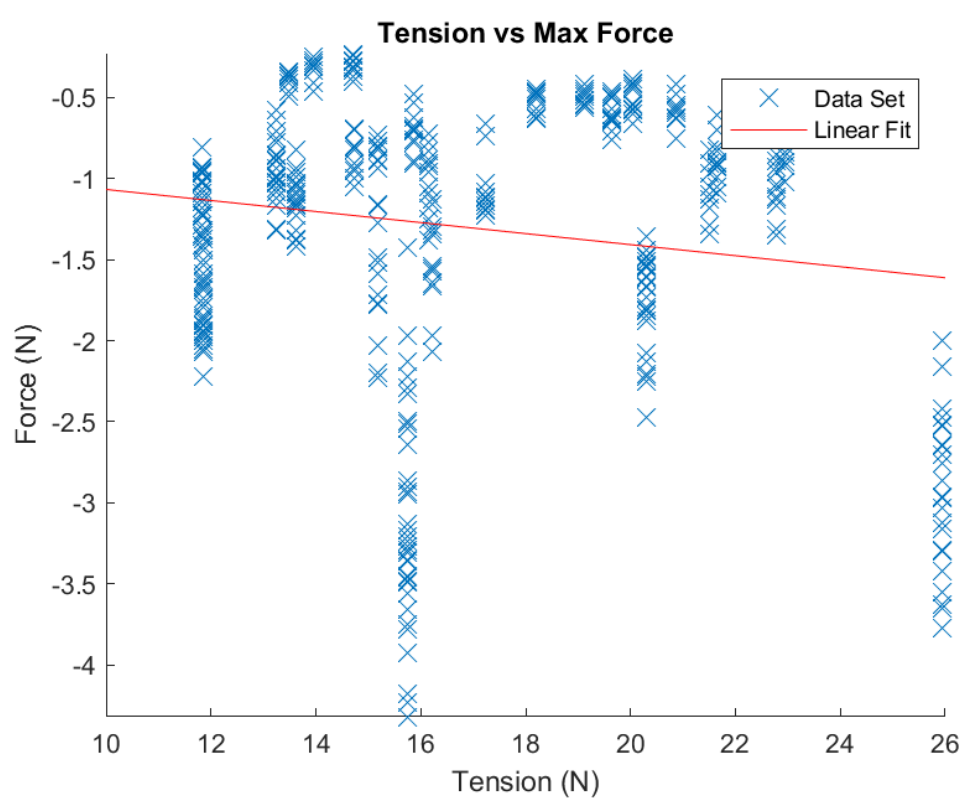

Figure 34: Measured maximum force plotted against applied tension; the red line shows the pair-wise relationship from a simple regression. Changes in tension were found to be significant $(p<0.05)$ in affecting the maximum force.

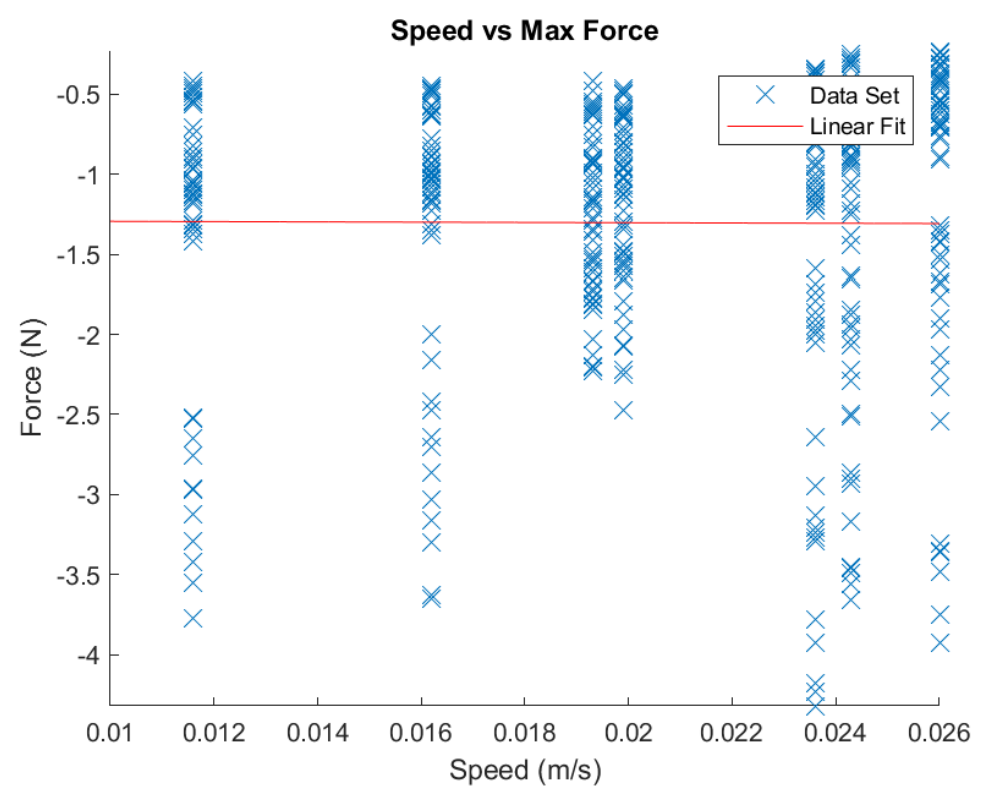

Figure 35: Measured maximum force plotted against insertion speed; the red line shows the pair-wise relationship from a simple regression. Speed was found to approach statistical significance $(p=0.056)$ in affecting the force. 


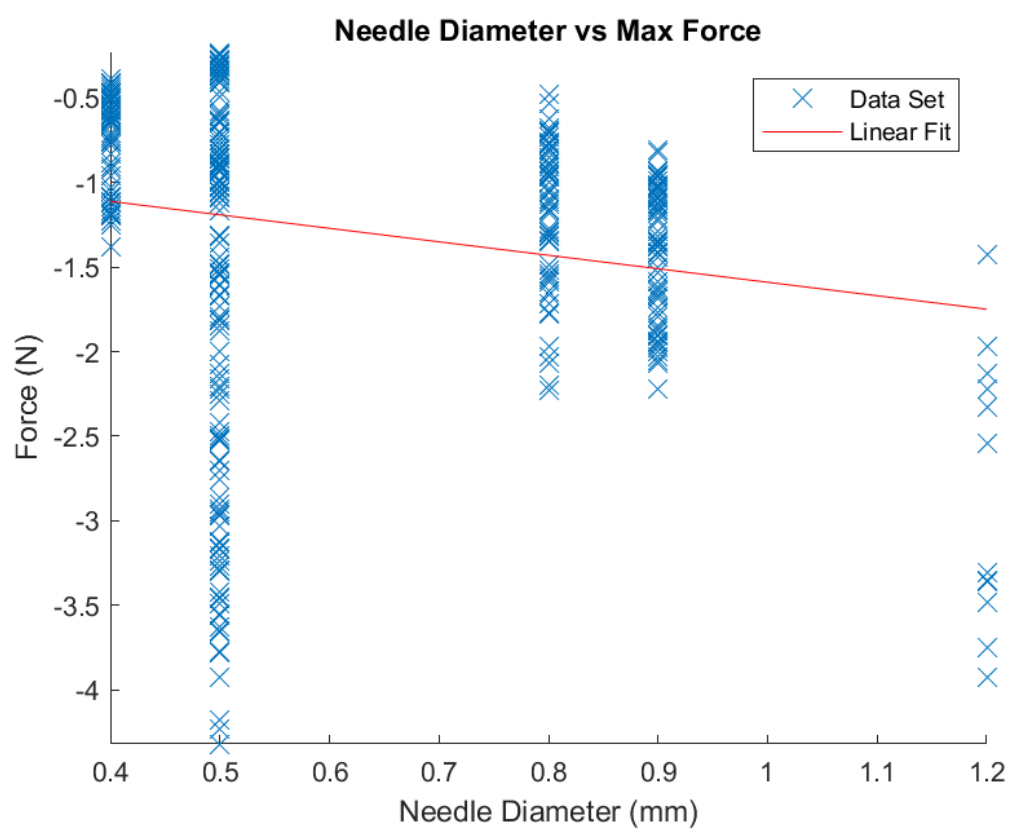

Figure 36: Measured maximum force plotted against needle diameter; the red line shows the pair-wise relationship from a simple regression. Changes in diameter were found to be significant $(\mathbf{p}<0.05)$ in affecting the maximum force.

\subsubsection{Fracture Toughness Coefficient Results}

The average calculated $\mathrm{J}_{\mathrm{IC}}$ of all the specimens was $2.17 \mathrm{~N} / \mathrm{m}$; the standard deviation was $1.68 \mathrm{~N} / \mathrm{m}(\mathrm{n}=420)$. The equation of the linear regression is given by Equation 14 . The $\mathrm{R}^{2}$ value of this fit was 0.12 indicating that variation in the results was poorly explained by the linear regression. Speed and needle diameter were not found to be statistically significant $(\mathrm{p}<0.05)$. Table 9 details the results of the statistical analysis. Figures 37, 38 and 39 show the maximum force plotted against each individual variable.

$$
J_{I C}=0.15 * F_{\text {tension }}-0.34
$$


Table 9: Step-wise regression results of the fracture toughness. Insertion speed and needle diameter were not significance as an explanatory variable $(p<0.05)$ and were omitted from the final regression equation. Applied skin tension was a significant independent variable $(p<0.05)$. The $R^{2}$ value of 0.12 indicates that tension has a weak relationship to fracture toughness.

\begin{tabular}{|l|l|l|l|l|l|}
\hline Variable & Coefficient & $\mathrm{p}$ value & Lower 95\% confidence & Upper 95\% confidence & Beta \\
\hline Tension & 0.15 & 0.000 & 0.083 & 0.14 & 0.35 \\
\hline Speed & 23.00 & 0.195 & -11.83 & 57.83 & \\
\hline Diameter & -0.68 & 0.094 & -1.72 & 0.12 & \\
\hline Constant & -0.34 & & -0.75 & 0.24 & \\
\hline
\end{tabular}

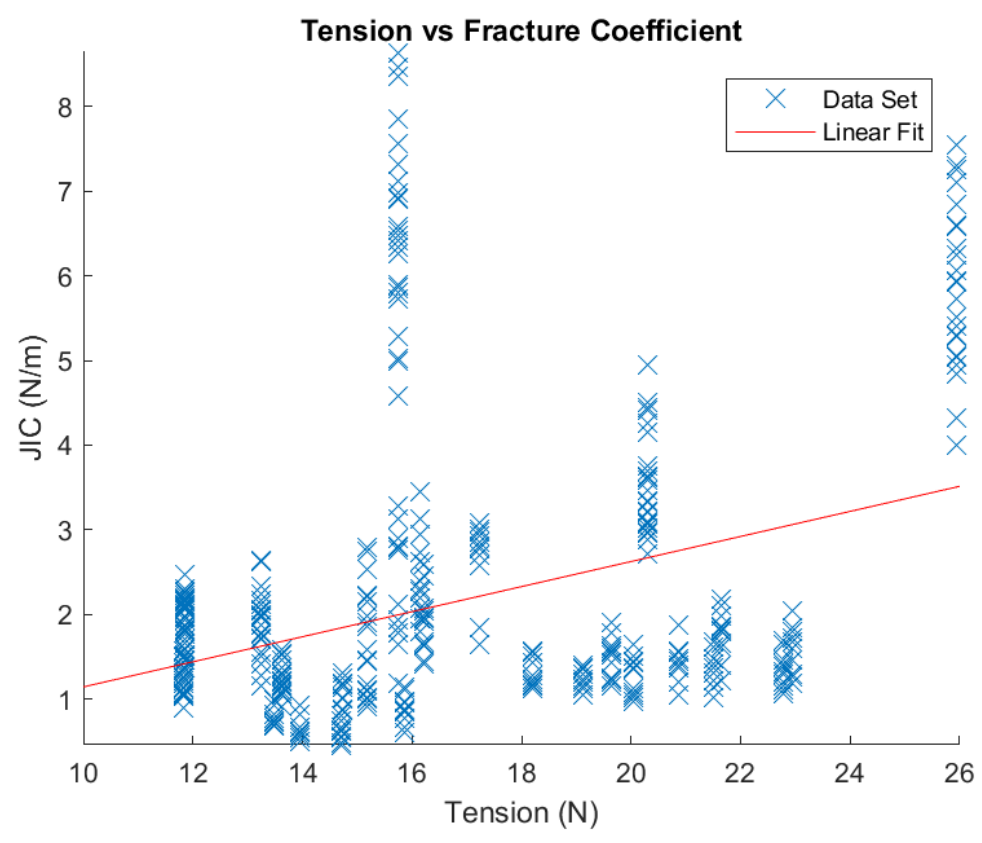

Figure 37: Measured fracture toughness coefficient plotted against applied tension; the red line shows the pair-wise relationship from a simple regression. Changes in tension were found to be significant $(p<0.05)$ in affecting the fracture coefficient. 


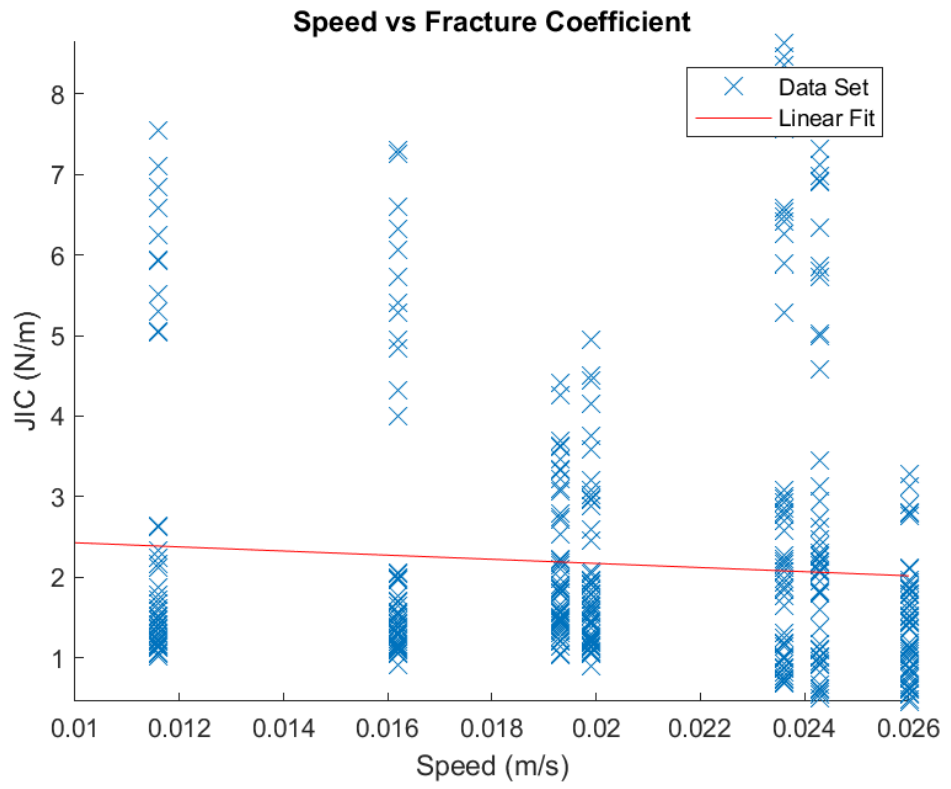

Figure 38: Measured fracture toughness coefficient plotted against insertion speed; the red line shows the pair-wise relationship from a simple regression. Changes in insertion speed were not found to be significant $(p=0.195)$ in affecting the fracture coefficient.

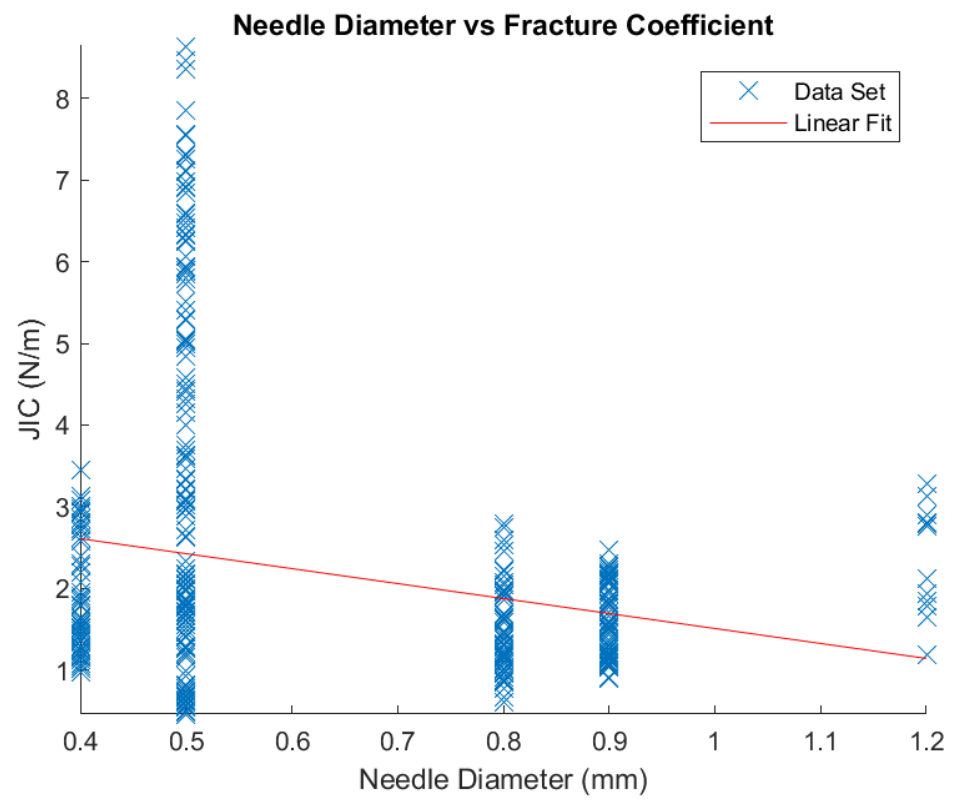

Figure 39: Measured fracture toughness coefficient plotted against needle diameter; the red line shows the pair-wise relationship from a simple regression. Changes in diameter were not found to be significant $(p=$ 0.195) in affecting the fracture coefficient. 


\subsection{Discussion}

This study derived an analytical model for determining the fracture toughness of porcine skin. It also identified independent variables that affects the fracture toughness and quantified their effect. The results presented in this paper have good agreement with experiments done on similar tissues. The average force results match closely the force results of needle insertion into porcine hearts and livers presented by Mahvash and Dupont [63]. The average also matches well with results given by Shergold and Fleck of in vivo testing on human forearm skin [65]. The result obtained do not match well with the results of Chu et al. [58]. Their results showed penetration forces of up of 20 to $30 \mathrm{~N}$ using porcine tissue from a pig's hindquarters. The results obtained by Chu et al. also differ significantly from the results of Naemura et al. and Liu et al. [60], [61]. Their experiments on porcine muscle tissue had maximum force results just above and below the ones presented in this paper; 2 to $3 \mathrm{~N}$ for Naemura et al. and 0.7 to $0.8 \mathrm{~N}$ for Liu et al. Deriving an explicit fracture coefficient has not been done in the literature reviewed. Given that the maximum force recorded was similar to other results in literature the fracture coefficient would be equally similar. This gives confidence that the trends observed in the data are applicable to porcine skin tissue and possibly also applicable to human skin tissue. Porcine tissue was used in this work due to research ethics and supply concerns for human tissue. Further testing using human skin tissue will be required to compare the results obtained with this experimental methodology.

The most interesting result seen was the lack of statistical significance of the effect changes in speed had on the recorded force and fracture coefficient. Tissue is a viscoelastic material, which means its force response is partially dictated by the applied strain rate. In another paper, which is in the process of being published; the maximum response force to indentation 
was found to have a weak but significant correlation to changes in indentation speed. This suggests that the force response to perpendicularly applied loads is only weakly viscoelastic. Higher recorded maximum force values were also recorded during indentation testing without damage to the surface tissue.

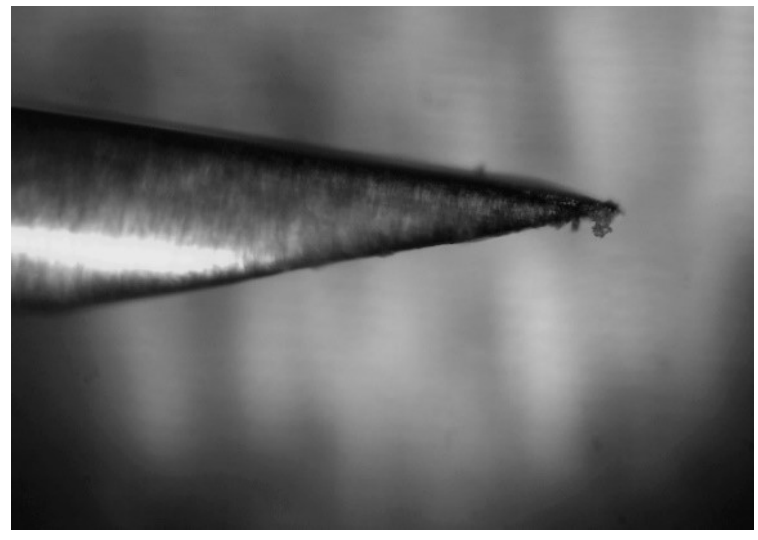

Figure 40: Tip geometry of hypodermic needle

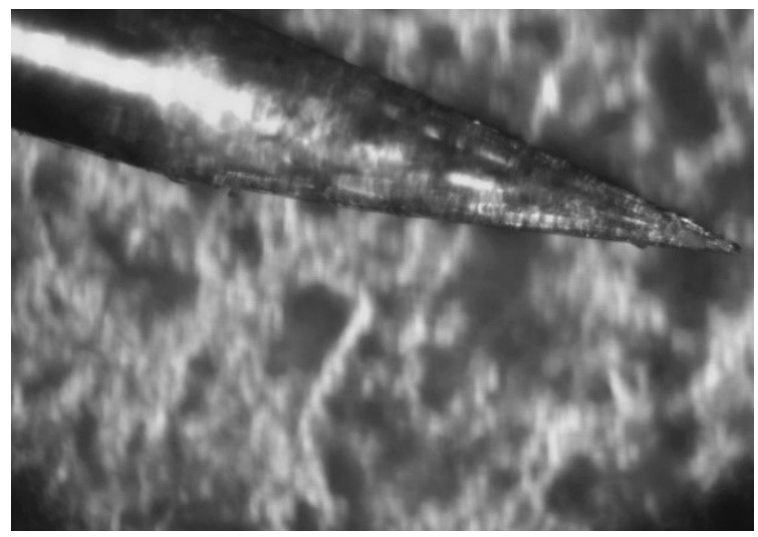

Figure 41: Tip geometry of the tattoo needle
The weak correlation between the linear regression fit and the experimental data for both the maximum force and the fracture coefficient indicates that other factors beyond the three investigated are important. The geometry of the needle tip seems to play a large role in the fracture properties of skin tissue. Preliminary testing was done using a stainless steel tattoo needle before switching to hypodermic needles. The tip geometries seen under a microscope are shown in Figures 40 and 41 . The tattoo needle was a point centered needle while the hypodermic needles were point offset. The tattoo needles, despite being made of the same material and the same diameter as the smallest hypodermic needle used, buckled without penetrating the surface skin layer.

Neither the "sharpness" or the tip configuration of the hypodermic needles were taken into account. It is known that the "sharpness" of the instrument impacts the fracture force [52]. O’Callaghan et al. were investigating a wide disparity of object from blunt to sharp. The 
hypodermic needles have the same basic configuration just different diameters. The slight changes in tip angle may have a larger effect than assumed.

It is also possible that there was significant variability with the skin surface layer of the samples themselves. Biological sample variability is a major hurdle in determining if the effect observed is a result of the independent variable or the material itself. As both linear regression fits have very low $\mathrm{R}^{2}$ values there is significant variability that is not explained by changes in the three variables studied. Previous studies and experiments have reported large between-sample variability. The sample variability of presented results in Ni Annaidh is 15 to 80 percent [9], Lagan is 10 to $40 \%$ [21]. Similar ranges are seen in work by Ottenio [10], Sandford [32], Mayrovitz [34], and others. Care was taken to ensure that the samples were as similar as possible from one test to the next, there was considerable variability within the samples. Samples might have a thicker or thinner fatty layer; samples might have fatty layers which appeared denser. Occasionally a muscle layer would be present immediately below the skin, other samples did not have any muscle layer. Obviously, these observed structural differences are significant. Assuming that approximately $90 \%$ of the variability is as a result of the sample as indicated by the $\mathrm{R}^{2}$ values is certainly unrealistic.

It is interesting that the skin tension significantly affected both the response force and the fracture coefficient. Skin tension was not found to significantly affect the indentation properties in a previous experiment. As the tension is increased the response force decreases (the force was measured as a negative value) and thus fracture coefficient increases (as a fracture coefficient is always positive). This results in the highest fracture force in scenarios with the most tension applied to the sample. This relationship does not make sense from a conceptual view. Higher skin tensions should act to "tear" the surface apart however, the results do not agree with this. It 
is also inconsistent with the assumption of a type I fracture mode. Increasing the far field stress and adding the same point force results in a higher stress field around the crack which would lead it to propagating at a longer point force. It is possible the higher applied skin tension activated more collagen fibers. This would remove the initial response portion ("toe" region) associated with low reaction force and large deformation. Once the collagen fibers are all activated the reaction force rapidly increases. The activation of more collagen fibers might explain the increase in force required to penetrate the skin surface at higher tensions. If there is low skin tension applied, when the surface of the skin deforms during the needle impact collagen fibers still need to fully activate and thus will allow large deformations with small reaction force. The same critical stress is reached around the needle point allowing skin rupture, but less skin surface area is resisting the deformation caused by the needle. As the skin tension increases the collagen fibers are already at or close to full activation thus the same amount of skin surface deformation requires more force to achieve.

It should be noted that in all cases, once the needle penetrated the surface layer of the skin, all perpendicular deflection rebounded, and the surface returned to its original level. Therefore, the work required to deflect the surface should be intrinsically tied to the fracture coefficient as there was no permanent deformation. However, previous indentation testing showed no correlation between skin tension and maximum reaction force.

The needle diameter was not shown to significantly affect the fracture coefficient; however, it was significant in the recorded reaction force. The beta values of skin tension and needle diameter are approximately equal. This could indicate that the skin tension, which may predict the surface shape during deformation, and the diameter of the needle are closely tied. The increase in fracture force with increasing needle diameter makes conceptual sense as there is a 
larger crack which needs to be initiated and propagated. It is however interesting that the needle diameter is not significant in predicting the fracture coefficient while it is significant in the recorded reaction force. Since the definition of fracture coefficient includes the needle diameter (by way of crack size) the effect of the crack size may have been eliminated.

\subsection{Conclusion}

Recorded maximum force values and calculated fracture coefficient were found to be weakly related to changes in skin tension and needle diameter. The effect of skin tension on the results matched the assumptions made, at higher skin tension values less force was required to fracture the skin tissue. Needle insertion speed was found to have no significant effect which contradicts the idea that skin tissue is a viscoelastic material. However, most of the variance in the experimental results was unexplained by the three independent variables investigated. 


\section{Chapter 4: Thesis Discussion}

The experimental work presented in Chapters 2 and 3 was intended to investigate the effect experimental variables have on changes in mechanical and fracture properties of skin tissue. The three independent variables chosen: skin tension, insertion or indentation speed, and tip geometry or needle diameter; were selected because conceptually they should have the greatest contributions to any change in properties. The experimental results agreed well with other results in literature and the trends of result variation conformed to many of the initial assumptions of the behaviour of skin tissue.

Although the motivation for this work was insertion of insulin injector set needles and cannula into the skin of human patients, this thesis used porcine tissue specimens. No literature has been found that directly compares porcine skin tissue to human skin tissue; however, porcine tissue has been used in other literature as a substitute for human tissue for the same reasons as these experiments. The differences between the two types of skin tissue may affect the material properties, however, it is likely that the material models developed in this work would also be appropriate for human skin. Whether the trends seen in the results and the interpretation of these trends and results is applicable to human skin is uncertain. Future testing is required to confirm this; but would require ethics clearance and a reliable supply of human skin samples.

In Section 2.5 the first relaxation period was interpreted to be the time required to balance the fluid pressure gradients within the skin tissue. The dermis layer is described as being mostly an "extra-cellular" matrix [5]. Based on this general description and other descriptions in literature [7], the extra-cellular matrix is not a solid medium but a fluidic space with many other molecules and cells suspended within it. This allows for fluid transfer from region to region 
which is impeded and channeled by the more solid components of this matrix. The physical restrictions and obstacles limit the amount of fluid which can pass out of a region in a unit time which allows the rise of localized high-pressure regions. Once the skin layer has been indented, the stress and strain region experiences this pressure gradient as a function of its radius out from the center of indentation. The relaxation periods are the denominator of the exponential as derived as Equation 10 in section 1.6, repeated below.

$$
\sigma=\epsilon\left(E_{\infty}+E_{1} e^{-\frac{t}{\tau_{1}}}+E_{2} e^{-\frac{t}{\tau_{2}}}\right)
$$

The first relaxation period being the smaller of the two, and a value less than one, will make the exponent approach infinity first and evaluate as zero. Mathematically this equates to a steeper curve, physically this means its effect dissipates rapidly. The second relaxation period was interpreted to be the relaxation time of the collagen fibers themselves. The fluidic contribution to the reaction force was interpreted to dissipate faster than the creep relaxation of the collagen fibers. The high-pressure regions will "inflate" the extra-cellular matrix which, conceptually, will add tensile loading to the collagen fibers slowing their creep relaxation. Once the pressure gradients have dissipated the collagen fibers will then be able to begin relaxing. For this reason, the short-term relaxation period has been interpreted to be the fluidic contribution and the long-term relaxation has been interpreted to be the collagen fiber contribution to the reaction force.

The linear regression fits for the maximum force, the first and second relaxation periods had poor correlation to the experimental values. All three had $\mathrm{R}^{2}$ values under 0.1 , meaning approximately $10 \%$ of the variance in results was explained by changes in the three independent variables: tip geometry, speed and skin tension. Results for initial modulus and long-term 
modulus presented in Sections 2.4.2 and 2.4.3 had regression coefficients $\left(\mathrm{R}^{2}\right)$ of 0.65 and 0.64. This indicates that there is approximately $35 \%$ variance not explained by changes in the three independent variables. Variability between samples is a property of biological materials and can be significant in affecting the results. However, it is unlikely that all this variance was caused by sample variability itself. There also exists some errors and uncertainties due to the experimental apparatus which will be detailed later. The estimate of the effect of sample variability is difficult to quantify and experimentally validate, thus any estimate used will be arbitrary. A larger data set, explicitly investigating sample variability will be required to have a more complete analysis of inherent sample variability. If the variance in the modulus results is used as a base estimate. It still leaves an approximate $50 \%$ variance in the experimental results for maximum force, first and second relaxation periods not explained by the three independent variables and the assumed samples variance. The maximum force was directly measured, and the two relaxation periods were calculated fitting parameters thus the missing factors for the variance are likely from different sources for each.

Skin is often thought of as a composite type material; type I fracture mechanics may not describe the fracture behaviour properly. Fracture of composite materials is complex and not yet as well defined as fracture of non-composites [51]. While the problem may appear to be similar to a type I fracture scenario the fracture of the individual collagen fibers at smaller scales may be better described using type II fracture mechanics. 


\subsection{Estimation of Sample Variability}

Appendix A shows figures of the maximum force results plotted against tension, holding speed and tip geometry or needle diameter constant. Each constant tension line is an individual sample, and there is large variation in results even within the same sample. The fracture force results have less variance than the indentation force results. The variance of the fracture results is on the order of 20 to $30 \%$ while the indentation variation is on the order of 40 to $60 \%$. With the approximate variance seen in the maximum force results, the estimate of sample variance of $35 \%$ in the moduli values is below the directly measured variance. As these moduli are fitting terms and depend on more than the peak force a direct comparison of these two estimates of variance is not valid. However, this does present a possible issue where variance attributed to one of the three independent variables may have been a result of sample variability or that a variable thought to not be statistically significant may actually be significant but be adversely affected by the inherent sample variance. Accounting for sample variability would be an empirical correction factor not an inherent material property, which makes its use in a generalized analytical model a poor idea. The goal of this research was to aid in the creation of a general model which could be applied to any skin tissue. Introducing species or sample location specific empirical correction factors defeats this purpose. It is possible that a general error factor could be derived that will reasonably account for sample variation across all types of skin tissue, but this would require significant resources and many types of experiments to collect enough data. 


\subsection{Source of Errors Within the Experimental Apparatus}

The variance seen might also be a result of errors within the testing apparatus. As the measured result, the reaction force profile is most affected by errors and issues with the experimental apparatus. The apparatus was a custom-built, three degrees of freedom frame; budgetary constraints meant a purpose-built actuated frame could not be purchased. Thus, there is a non negligible amount of compliance and nonorthogonality with the positioning within the frame. This deficiency was mitigated as best as possible using good fabrication practices and checking the frame and instrumentation with a level during each test, but it does add some uncertainty to the results. The testing and instrumentation frames were constructed of steel and aluminum; there is negligible deflection of these members at the force levels used during testing. The requirement to allow for freedom of movement in all three axis means there is the possibility of shifting or sliding at the connection points of the frames. Effort was made to ensure the line of indentation was perpendicular to the surface of the tissue, however, given the imperfectness of both the frame and the porcine samples this was impossible to achieve.

Sections 1.7 and 2.2 have an assumption of a fixed ratio of indentation depth to strain affected radius; this ratio of 1.67 was taken from literature which used simulated tissues. This assumption was made because direct measurement of the deflected surface was not undertaken in the experiments done for this thesis. Using direct image correlation or other imaging based tool to measure the deflection would have been difficult to achieve given the experimental set-up.

The indenter tip displacement was set to $2.5 \mathrm{~mm}$ below the surface of the skin, this was done by measuring the distance at the test location by hand with a digital caliper. The accuracy on the input into the linear actuator was also not excellent with an accuracy of approximately 0.1 $\mathrm{mm}$. Thus, the accepted range of inputs into the linear actuator was indentation between 2.4 and 
$2.6 \mathrm{~mm}$ from the measured surface. In addition, during certain trials the skin tissue obviously shifted. When the $2.5 \mathrm{~mm}$ indentation was set, the tip hardly indented the surface of the skin requiring a remeasuring and repeating of that trial. The inline load cell (LCMFL-20N) has a rated accuracy of $0.1 \mathrm{~N}$, with the rated zero balance of $0.4 \mathrm{~N}$. The noise on the amplifier board is negligible, with a rated input noise of $90 \mathrm{nV}$, where the load cell supplies $0.5 \mathrm{mV} / \mathrm{N}$. It is difficult to quantify the effect these inaccuracies in apparatus and indentation depth tolerance had on the experimental results.

The data collection rate also likely produced errors, the load cells sent their signal to an amplifier which also converted the analog signal to a digital signal. This meant the data collection rate was one sample point every ninety milliseconds. With speeds between $0.0116 \mathrm{~m} / \mathrm{s}$ and $0.0260 \mathrm{~m} / \mathrm{s}$ the $2.5 \mathrm{~mm}$ indentation would take between 96 and 215 millisecond or 1 and 2 sample points. Many attempts were made to increase the reading rate of the system, however the need to amplify the load cell signal and the amplifier's conversion from an analog to a digital signal meant that it was impossible to make the data acquisition rate any faster. The peak of the data was possibly missed in some trials which probably accounted for some of the large variance seen in the maximum force values.

The data acquisition rate also can explain some of the variance in the first and second relaxation periods. Including the missing peak data might have allowed for better data fits which could have reduced the variance in the two fitting terms. It is also possible the level of hydration in the porcine tissue may have contributed to the variance. The skin was wrapped in soaked paper towels for a minimum of two hours prior to commencement of testing and was tested within an hour and a half of unwrapping. There was not a noticeable difference in tissue hydration from beginning to end of the test but the amount of water in the extra-cellular matrix 
may have decreased during the testing process. Since the interpretation of the first relaxation period is a fluidic contribution the decrease in the amount of water would lessen the localized high-pressure regions. This would change the value of this first fitting term as the peak may not be as high as at maximum hydration and the gradients would dissipate quicker due to the decrease in total fluid volume. This would also allow the collagen fibers to begin to relax quicker which would affect the second fitting term.

The skin tension was found to have a minimal effect on the change in indentation response properties. The porcine sample was sutured into a metal bracket approximately 5.5 inches in length, then tensioned through a central rod. It is possible the tension within the entirety of the sample was not uniform; that the central areas of the tissue experienced a reduced tensile force than areas near to the perimeter.

The errors and uncertainties within the skin fracture experiment are the same as in the indentation experiment. The possibility of missing the peak force during data collection is the largest uncertainty since the reaction force sharply drops off once the skin in pierced compared to the indentation testing, which is ramp and hold. The imperfect orthogonality of the apparatus likely had a greater contribution in errors than in the indentation testing. The point of the needle being even slightly angled would increase the surface area trying to pierce the skin which would effectively lessen the stress concentrations around the tip. This could also introduce flexing in the needle shaft which would shift the line of force off center and could have been read incorrectly by the load cell. 


\subsection{Comparing the Experimental Apparatus to the Manufactured Infusion Kit}

The real infusion kit was investigated early in the experiment design process to derive the parameters for testing. A spring constant of approximately $800 \mathrm{~N} / \mathrm{m}$ was measured in devices that had been used several times, likely not measuring the spring constant of a new device. The device was able to retract $17.5 \mathrm{~mm}$ from its original position, the needle was $9 \mathrm{~mm}$ long. Total spring energy was calculated at approximately $0.1 \mathrm{~J}$. Once released the device would travel 8.5 $\mathrm{mm}$ before the needle would impact the surface. Given an approximate mass of $5 \mathrm{~g}$, the needle point would be travelling at approximately $0.69 \mathrm{~m} / \mathrm{s}$. The linear actuator could only produce a speed of $0.0260 \mathrm{~m} / \mathrm{s}$, no actuator could produce the required speed with the accuracy that would be required to do the testing. For this reason, position and speed accuracy were chosen over obtaining comparable speeds.

The results of the experiments were to be used to develop energy requirements for each stage of the insertion process and compared to the available energy in the springs of approximately $0.1 \mathrm{~J}$. The cannula could be approximated as a cylindrical indenter which is why that type of tip was chosen. It would also be a relatively simple manufacturing addition to chamfer the edge of the cannula which could be approximated by the conical indenters.

At the impact of the needle on the tissue the force would be approximately $7 \mathrm{~N}$, based on the results presented in the Chapter 3 this amount of force would easily penetrate porcine skin. After an additional $3 \mathrm{~mm}$ the cannula would impact the surface with a force of approximately 4 $\mathrm{N}$ which would also be sufficient to deform the skin surface $2.5 \mathrm{~mm}$, although, the cannula will have likely penetrated the surface layer before that point. However, as the results in Chapter 2 show, the reaction force increases with increased speed, while the decrease of contact surface area has a smaller contribution. During some preliminary testing with previously used infusion 
kits, the cannula had difficulty fully penetrating the porcine skin. This suggests that the results and trends seen in Chapter 2 continue for speeds far exceeding those used during the experiment. Therefore, it might be advantageous to find a method to reduce the impact speed of the infusion kit. However, further testing using unused infusion kits will be required to confirm this.

Samples were tested at room temperature (approximately $22^{\circ} \mathrm{C}$ ), while human core body temperature is approximately $37^{\circ} \mathrm{C}$, with the skin surface being somewhat lower. The effect of temperature was not investigated during these experiments but could have an effect on the observed properties. However, given that human body temperature remains relatively constant the effect due to temperature would be of minimal impact of the design of the infusion kit.

Each of the three independent variables has an effect on different properties; skin tension has an effect on fracture properties and speed and tip geometry have an effect on the indentation response. This means all the variables must be considered when designing upgrades or changes to the infusion kits. Testing of the combined set-up, indentation and fracture, will be required to determine which of the independent variables have a larger effect on the overall insertion process. 


\section{Chapter 5: Thesis Conclusion}

The results presented in this thesis had good agreement with some results presented in literature. Literature explicitly investigating the effect outside variables have on the mechanical and fracture properties of tissue has not been found, thus the trends presented here cannot be compared. However, given the good correlation between the average results and the literature, the trends can be accepted as a preliminary base for other work to be done.

The results presented in the Chapter 2 confirmed the basic assumption of skin tissue being viscoelastic, the rate of applied strain had a significant effect on all the properties. The experiments also produced some interesting results. First, skin tension has a negligible effect on the mechanical properties on skin tissue. Second, the three identified outside variables poorly explained the variance in the maximum force results and the calculated two relaxation periods.

The results presented in the Chapter 3 produced the opposite result to the indentation experiments. Skin tension was found to be significant in affecting both the maximum recorded force and the fracture toughness coefficient. However, the variance in recorded results had poor correlation to the three outside variables indicating that other variables have more effect. 


\section{Chapter 6: Contributions to the Field}

- Developed a material model describing the reaction of skin tissue to indentation comparable to that presented in literature. Used it to derive material properties and quantified the effect changes in applied skin tension, indentation speed and indenter tip geometry had on the measured material properties. Indentation speed and indenter tip geometry were significant in affecting the measured properties.

- Presented new material modulus results that compare well to others in literature.

- Developed a material model describing fracture properties of skin tissue comparable to that presented in literature. Used it to derive a fracture coefficient and quantified the effect changes in applied skin tension, insertion speed and needle diameter had on the measured fracture toughness. Skin tension was significant in affecting the measured properties. 


\section{Chapter 7: Future Work}

The results and derivations presented in this thesis will allow for further investigations into the real infusion kit. As stated in Section 1.11 the original goal of this thesis was to create a model for the potential of damage to the cannula during the insertion process. Section 1.11 introduced some of the concepts that would be required to create this model. Using the work presented in this thesis, a basis for estimating the force applied to the free end of the cannula may be developed. Before the insertion of the central needle into the skin tissue there are two distinct phases to the model. Initial skin deflection and fracture of the skin tissue; the work presented here allows for estimation of both of these parameters accounting for varying tip geometries and insertion speeds. Once the skin fractures and the central needle is allowed to penetrate into the underlying skin layers the cannula will come into contact with the skin surface. At this point the skin will again deform both parallel and perpendicular to the path of the cannula.

The deflection of the skin surface parallel to the needle path may be modeled using the results of the indentation of the skin in Chapter 2. This will allow the derivation of the amount of force applied to the end of the cannula, which will allow the buckling estimation and calculation to occur. Additional experiments will need to be performed using an existing crack by a central needle to adjust the equations obtained in Chapter 3 . The deformation profile will likely change as a result of the rupture in the skin tissue which should lessen the force applied to the end of the cannula. Depending on the results of that work, using an "unbroken" skin analysis might be reasonable to allow for an inherent safety factor.

Testing of actual manufactured infusion kits will also be necessary to allow for model correlation and correction. The main issue is the measurement of forces or surface locations. 
Having the ability to measure the force on the cannula and needle without fundamentally changing how the system works is the largest challenge that would need to be overcome. Using a load cell to measure the spring force during retraction of the device to derive an energy system was the first experimental design conceived. It would, however, not allow for measurements during the insertion phase, it would only allow inferences to be made based on the final resting configuration of the system. 


\section{Chapter 8: References}

[1] "Statistics Canada." [Online]. Available: https://www150.statcan.gc.ca/t1/tb11/en/cv.action?pid=1310009601. [Accessed: 19-Feb-2020].

[2] "Diabetes Canada." [Online]. Available: https://www.diabetes.ca/. [Accessed: 19-Feb-2020].

[3] P. L. Ross, J. Milburn, D. M. Reith, E. Wiltshire, and B. J. Wheeler, "Clinical review: insulin pumpassociated adverse events in adults and children," Acta Diabetol., vol. 52, no. 6, pp. 1017-1024, 2015.

[4] P. J. Patel et al., "Randomized trial of infusion set function: Steel versus teflon," Diabetes Technol. Ther., vol. 16, no. 1, pp. 15-19, 2014.

[5] Various, Lippincott Professional Guides : Anatomy \& Physiology, Second. Lippincott Williams \& Wilkins, 2002.

[6] G. Limbert, Skin Biophysics. Springer International Publishing, 2019.

[7] C. Ross Ethier and Craig A. Simmons, Introductory Biomechanics From Cells to Organisms. .

[8] V. C. Mow and W. C. Hayes, Basic Orthopaedic Biomechanics, Second Edi. Lippincott-Raven, 1997.

[9] A. Ní Annaidh, K. Bruyère, M. Destrade, M. D. Gilchrist, and M. Otténio, "Characterization of the anisotropic mechanical properties of excised human skin,” J. Mech. Behav. Biomed. Mater., vol. 5, no. 1, pp. 139-148, 2012.

[10] M. Ottenio, D. Tran, A. Ní Annaidh, M. D. Gilchrist, and K. Bruyère, "Strain rate and anisotropy effects on the tensile failure characteristics of human skin," J. Mech. Behav. Biomed. Mater., vol. 41, pp. 241-250, 2015.

[11] Z. L. Shen, H. Kahn, R. Ballarini, and S. J. Eppell, "Viscoelastic properties of isolated collagen fibrils," Biophys. J., vol. 100, no. 12, pp. 3008-3015, 2011.

[12] W. C. Hayes, L. M. Keer, G. Herrmann, and L. F. Mockros, "A mathematical analysis for indentation tests of articular cartilage," J. Biomech., vol. 5, no. 5, pp. 541-551, 1972.

[13] P. Julkunen, R. K. Korhonen, W. Herzog, and J. S. Jurvelin, "Uncertainties in indentation testing of articular cartilage: A fibril-reinforced poroviscoelastic study," Med. Eng. Phys., vol. 30, no. 4, pp. 506-515, 2008.

[14] J. Gosline et al., "Elastic Proteins : Biological Roles and Mechanical Properties and Ken Savage Source : Philosophical Transactions: Biological Sciences, Vol .357, No . 1418, Elastomeric Proteins : Structures , Biomechanical Properties and Biological Roles ( Feb . 28 ," vol. 357, no. 1418, 2016.

[15] A. K. Dabrowska et al., "Materials used to simulate physical properties of human skin," Ski. Res. Technol., vol. 22, no. 1, pp. 3-14, 2016.

[16] N. Kumaraswamy, H. Khatam, G. P. Reece, M. C. Fingeret, M. K. Markey, and K. Ravi-Chandar, "Mechanical response of human female breast skin under uniaxial stretching," J. Mech. Behav. Biomed. Mater., vol. 74, no. May, pp. 164-175, 2017.

[17] F. Silver, J. Freeman, and D. DeVore, "Viscoelastic properties of human skin and processed dermis.," Ski. Res. Technol., vol. 7, no. 1, pp. 18-23, 2001.

[18] J. E. Bischoff, E. M. Arruda, and K. Grosh, "Finite element modeling of human skin using an isotropic, nonlinear elastic constitutive model," J. Biomech., vol. 33, no. 6, pp. 645-652, 2000.

[19] S. L. Evans and C. A. Holt, "Measuring the mechanical properties of human skin in vivo using digital image correlation and finite element modelling," J. Strain Anal. Eng. Des., vol. 44, no. 5, pp. 337-345, 2009.

[20] G. Fang and A. Köppl, "FEM simulation of single beard hair cutting with foil-blade-shaving system," $J$. Mech. Behav. Biomed. Mater., vol. 46, no. November, pp. 271-284, 2015.

[21] S. D. Łagan and A. Liber-Kneć, "Experimental testing and constitutive modeling of the mechanical properties of the swine skin tissue," Acta Bioeng. Biomech., vol. 19, no. 2, pp. 93-102, 2017.

[22] P. A. L. S. Martins, R. M. N. Jorge, and A. J. M. Ferreira, "A comparative study of several material models for prediction of hyperelastic properties: Application to silicone-rubber and soft tissues," Strain, vol. 42, no. 3, pp. 135-147, 2006.

[23] G. A. Holzapfel, Nonlinear solid mechanics : a continuum approach for engineering. 2000.

[24] D. R. Veronda, H. G. Systems, R. A. Westmann, A. Science, and L. Angeles, "MECHANICAL CHARACTERIZATION SKIN-FINITE DEFORMATIONS," vol. 3, no. I 965, 1970.

[25] J. D. Humphrey, "FINITE EXTENSION AND TORSION OF PAPILLARY MUSCLES : A THEORETICAL FRAMEWORK," vol. 25, no. 5, pp. 541-547, 1992.

[26] J. D. Humphrey, "Continuum biomechanics of soft biological tissues," 2003. 
[27] D. Remache, M. Caliez, M. Gratton, and S. Dos Santos, "The effects of cyclic tensile and stress-relaxation tests on porcine skin," J. Mech. Behav. Biomed. Mater., vol. 77, no. August 2017, pp. 242-249, 2018.

[28] A. Karimi, M. Navidbakhsh, M. Haghighatnama, and A. M. Haghi, "Determination of the axial and circumferential mechanical properties of the skin tissue using experimental testing and constitutive modeling," Comput. Methods Biomech. Biomed. Engin., vol. 18, no. 16, pp. 1768-1774, 2015.

[29] S. Nicolle, J. Decorps, B. Fromy, and J. F. Palierne, "New regime in the mechanical behavior of skin: strainsoftening occurring before strain-hardening," J. Mech. Behav. Biomed. Mater., vol. 69, no. October 2016, pp. 98-106, 2017.

[30] C. Then, B. Stassen, K. Depta, and G. Silber, "New methodology for mechanical characterization of human superficial facial tissue anisotropic behaviour in vivo," J. Mech. Behav. Biomed. Mater., vol. 71, no. February, pp. 68-79, 2017.

[31] E. Jacquet, J. Chambert, J. Pauchot, and P. Sandoz, "Intra- and inter-individual variability in the mechanical properties of the human skin from in vivo measurements on 20 volunteers," Ski. Res. Technol., vol. 23, no. 4, pp. 491-499, 2017.

[32] E. Sandford, Y. Chen, I. Hunter, G. Hillebrand, and L. Jones, "Capturing skin properties from dynamic mechanical analyses," Ski. Res. Technol., vol. 19, no. 1, pp. 339-348, 2013.

[33] T. Virén, J. T. Iivarinen, J. K. Sarin, I. Harvima, and H. N. Mayrovitz, "Accuracy and reliability of a handheld in vivo skin indentation device to assess skin elasticity," Int. J. Cosmet. Sci., vol. 40, no. 2, pp. 134140, 2018.

[34] H. N. Mayrovitz, K. Corbitt, A. Grammenos, A. Abello, and J. Mammino, "Skin indentation firmness and tissue dielectric constant assessed in face, neck, and arm skin of young healthy women," Ski. Res. Technol., vol. 23, no. 1, pp. 112-120, 2017.

[35] H. V. Tran, F. Charleux, M. Rachik, A. Ehrlacher, and M. C. Ho Ba Tho, "In vivo characterization of the mechanical properties of human skin derived from MRI and indentation techniques," Comput. Methods Biomech. Biomed. Engin., vol. 10, no. 6, pp. 401-407, 2007.

[36] R. B. Groves, S. A. Coulman, J. C. Birchall, and S. L. Evans, "Quantifying the mechanical properties of human skin to optimise future microneedle device design," Comput. Methods Biomech. Biomed. Engin., vol. 15 , no. 1, pp. 73-82, 2012.

[37] I. N. Sneddon, "The relation between load and penetration in the axisymmetric boussinesq problem for a punch of arbitrary profile," Int. J. Eng. Sci., vol. 3, no. 1, pp. 47-57, 1965.

[38] C. Pailler-Mattei, S. Bec, and H. Zahouani, "In vivo measurements of the elastic mechanical properties of human skin by indentation tests," Med. Eng. Phys., vol. 30, no. 5, pp. 599-606, 2008.

[39] J. Isaza and J. Ramirez, "Incidence of temperature and indenter diameter on the mechanical response of skin during indentation test," Procedia Eng., vol. 110, pp. 45-50, 2015.

[40] R. Álvarez-Asencio et al., "Nanomechanical properties of human skin and introduction of a novel hair indenter," J. Mech. Behav. Biomed. Mater., vol. 54, pp. 185-193, 2016.

[41] A. N. Dai et al., "In vivo adhesive behavior of human facial skin by a modified indentation test," J. Mech. Behav. Biomed. Mater., vol. 92, no. January, pp. 172-178, 2019.

[42] C.-Y. Chen, C.-A. Yu, T.-F. Hong, Y.-L. Chung, and W.-L. Li, "Contact and frictional properties of stratum corneum of human skin," Biosurface and Biotribology, vol. 1, no. 1, pp. 62-70, 2015.

[43] J. Jachowicz, R. Mcmullen, and D. Prettypaul, "Indentometric analysis of in vivo skin and comparison with artificial skin models," Ski. Res. Technol., vol. 13, no. 3, pp. 299-309, 2007.

[44] R. M. Christensen, Theory of Viscoelaticity, Second Edi. Academic Press, 1982.

[45] Y. Liu, Y. Wei, and P. Chen, "Indentation response of soft viscoelastic matter with hard skin," Soft Matter, vol. 15, no. 28, pp. 5760-5769, 2019.

[46] S. J. M. Yazdi, K. S. Cho, and N. Kang, "Characterization of the viscoelastic model of in vivo human posterior thigh skin using ramp-relaxation indentation test," Korea Aust. Rheol. J., vol. 30, no. 4, pp. 293$307,2018$.

[47] J. Simsiriwong, R. W. Sullivan, and H. H. Hilton, "Challenges in Mechanics of Time-Dependent Materials, Volume 2: Proceedings of the 2014 Annual Conference on Experimental and Applied Mechanics," vol. 2, no. 1, p. 196, 2014.

[48] D. Gao, Y. Lei, and B. Yao, "Dynamic soft tissue deformation estimation based on energy analysis," Chinese J. Mech. Eng. (English Ed., vol. 29, no. 6, pp. 1167-1175, 2016.

[49] W. D. Pilkey, Formulas for Stress, Strain, and Structural Matrices: Second Edition. 2008. 
[50] D. Gross and T. Seelig, Fracture mechanics: With an introduction to micromechanics, vol. 53. 2006.

[51] A. Shukla, Practical Fracture Mechanics in Design, Second. CRC Press, 2004.

[52] S. L. P.T. O'Callaghana, M.D. Jones, D.S. James and L. D. M. N. C.A. Holt, "Dynamics of stab wounds: force required for penetration of various cadaveric human tissues," Forensic Sci. Int., vol. 104, no. 2-3, pp. 173-178, 1999.

[53] A. Ní Annaidh, M. Cassidy, M. Curtis, M. Destrade, and M. D. Gilchrist, "A combined experimental and numerical study of stab-penetration forces," Forensic Sci. Int., vol. 233, no. 1-3, pp. 7-13, 2013.

[54] J. Ling et al., "Insertion and Pull Behavior of Worker Honeybee Stinger," J. Bionic Eng., vol. 13, no. 2, pp. 303-311, 2016.

[55] J. Ling et al., "Effect of honeybee stinger and its microstructured barbs on insertion and pull force," J. Mech. Behav. Biomed. Mater., vol. 68, no. August 2016, pp. 173-179, 2017.

[56] S. Aoyagi, H. Izumi, and M. Fukuda, "Biodegradable polymer needle with various tip angles and consideration on insertion mechanism of mosquito's proboscis," Sensors Actuators, A Phys., vol. 143, no. 1, pp. 20-28, 2008.

[57] J. Kim, S. Park, G. Nam, Y. Choi, S. Woo, and S. H. Yoon, "Bioinspired microneedle insertion for deep and precise skin penetration with low force: Why the application of mechanophysical stimuli should be considered," J. Mech. Behav. Biomed. Mater., vol. 78, no. October 2017, pp. 480-490, 2018.

[58] J. P. Chu, N. Bönninghoff, C. C. Yu, Y. K. Liu, and G. H. Chiang, "Coating needles with metallic glass to overcome fracture toughness and trauma: Analysis on porcine tissue and polyurethane rubber," Thin Solid Films, vol. 688, no. May, p. 137320, 2019.

[59] S. Misra, K. B. Reed, B. W. Schafer, K. T. Ramesh, and A. M. Okamura, "Mechanics of flexible needles robotically steered through soft tissue," Int. J. Rob. Res., vol. 29, no. 13, pp. 1640-1660, 2010.

[60] W. Liu, Z. Yang, P. Li, J. Zhang, and S. Jiang, "Mechanics of tissue rupture during needle insertion in transverse isotropic soft tissue," Med. Biol. Eng. Comput., vol. 57, no. 6, pp. 1353-1366, 2019.

[61] K. Naemura, Y. Matsumoto, and H. Saito, "Effect of young's modulus of porcine ligamentum flavum on the epidural needle insertion," J. Eng., vol. 2019, no. 14, pp. 473-477, 2019.

[62] M. Heverly, P. Dupont, and J. Triedman, "Trajectory optimization for dynamic needle insertion," Proc. IEEE Int. Conf. Robot. Autom., vol. 2005, no. April, pp. 1646-1651, 2005.

[63] M. Mahvash and P. E. Dupont, "Mechanics of dynamic needle insertion into a biological material," IEEE Trans. Biomed. Eng., vol. 57, no. 4, pp. 934-943, 2010.

[64] F. Casanova, P. R. Carney, and M. Sarntinoranont, "In vivo evaluation of needle force and friction stress during insertion at varying insertion speed into the brain," J. Neurosci. Methods, vol. 237, pp. 79-89, 2014.

[65] O. A. Shergold and N. A. Fleck, "Experimental Investigation Into the Deep Penetration of Soft Solids by Sharp and Blunt Punches, With Application to the Piercing of Skin," J. Biomech. Eng., vol. 127, no. 5, p. $838,2005$.

[66] O. A. Shergold and N. A. Fleck, "Mechanisms of deep penetration of soft solids, with application to the injection and wounding of skin," Proc. R. Soc. A Math. Phys. Eng. Sci., vol. 460, no. 2050, pp. 3037-3058, 2004.

[67] M. J. Oldfield et al., "Highly resolved strain imaging during needle insertion: Results with a novel biologically inspired device," J. Mech. Behav. Biomed. Mater., vol. 30, pp. 50-60, 2014.

[68] S. F. Han and Y. Yang, "Influence of needling conditions on the corneal insertion force," Comput. Methods Biomech. Biomed. Engin., vol. 22, no. 16, pp. 1239-1246, 2019.

[69] J. H. Michell, "Some Elementary Distributions of Stress in Three Dimensions.," Proc. London Math. Soc., vol. 32.1, pp. 23-61, 1900.

[70] A. Leissa, Vibration of Shells, NASA SP-28. Scientific and Technical Information Office National Aeronautics and Space Administration, 1973.

[71] S. P. Timoshenko and J. M. Gere, Theory of Elastic Stability, Second Edi. DOVER PUBLICATIONS, INC., 1961.

[72] M. D. Parker, L. A. Jones, I. W. Hunter, A. J. Taberner, M. P. Nash, and P. M. F. Nielsen, "Multidirectional in Vivo Characterization of Skin Using Wiener Nonlinear Stochastic System Identification Techniques," $J$. Biomech. Eng., vol. 139, no. 1, pp. 1-11, 2017. 


\section{Appendix A - Supplementary Figures}

This appendix contains supplementary figures for use in Chapter 4 for estimation of the amount of sample variation within the experiment. Each figure shows a specific tip and speed with constant tension lines.
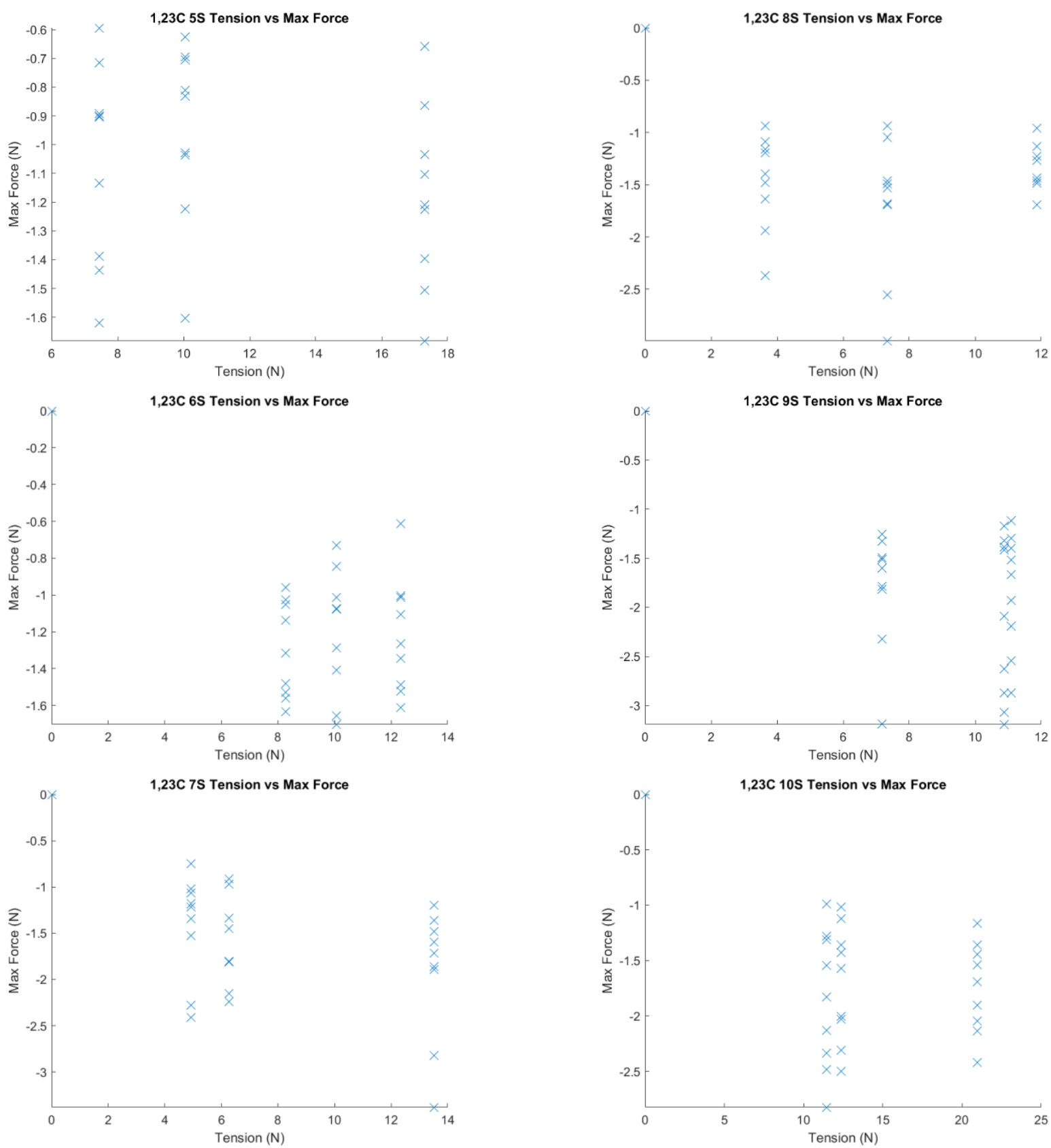

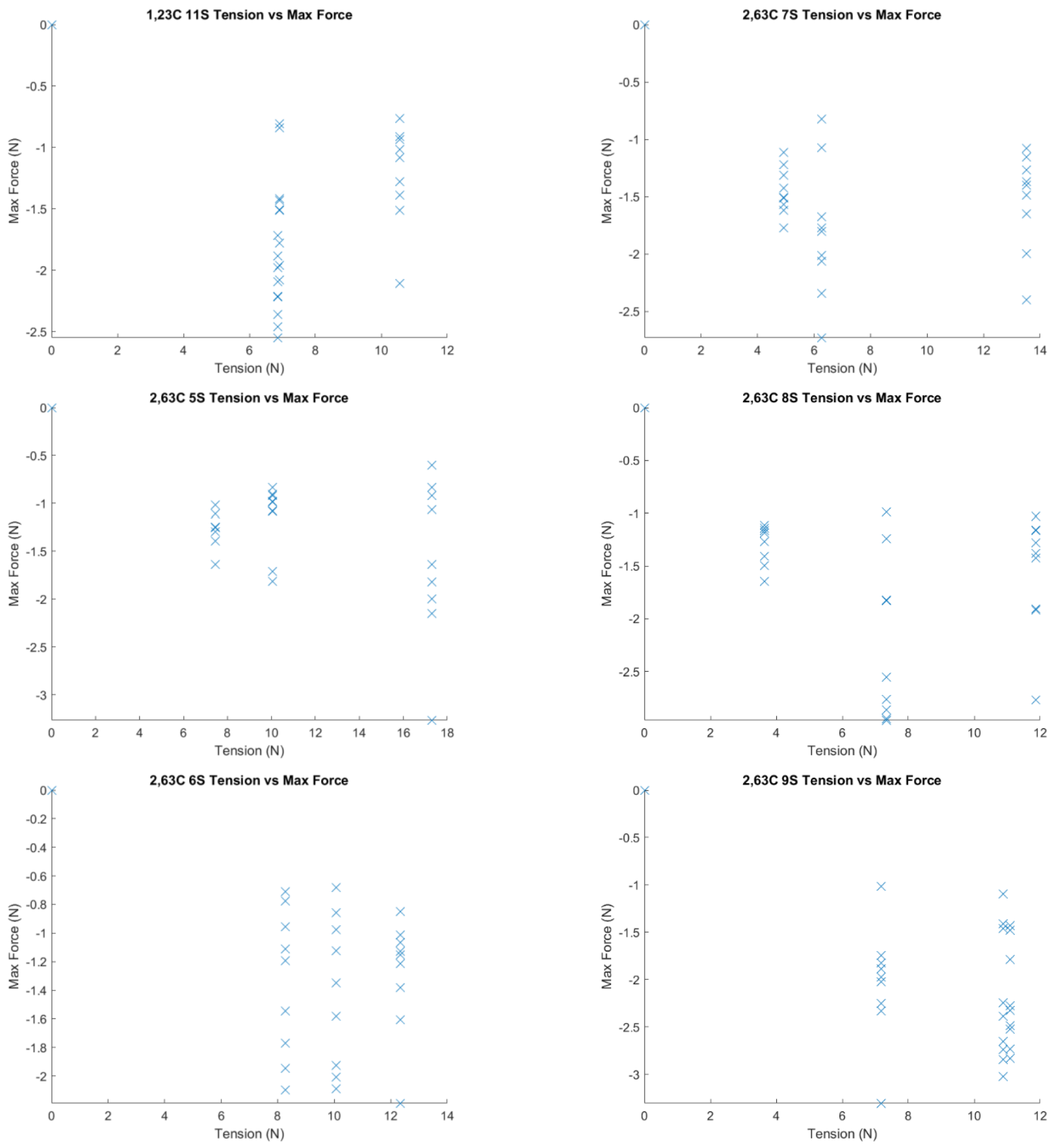

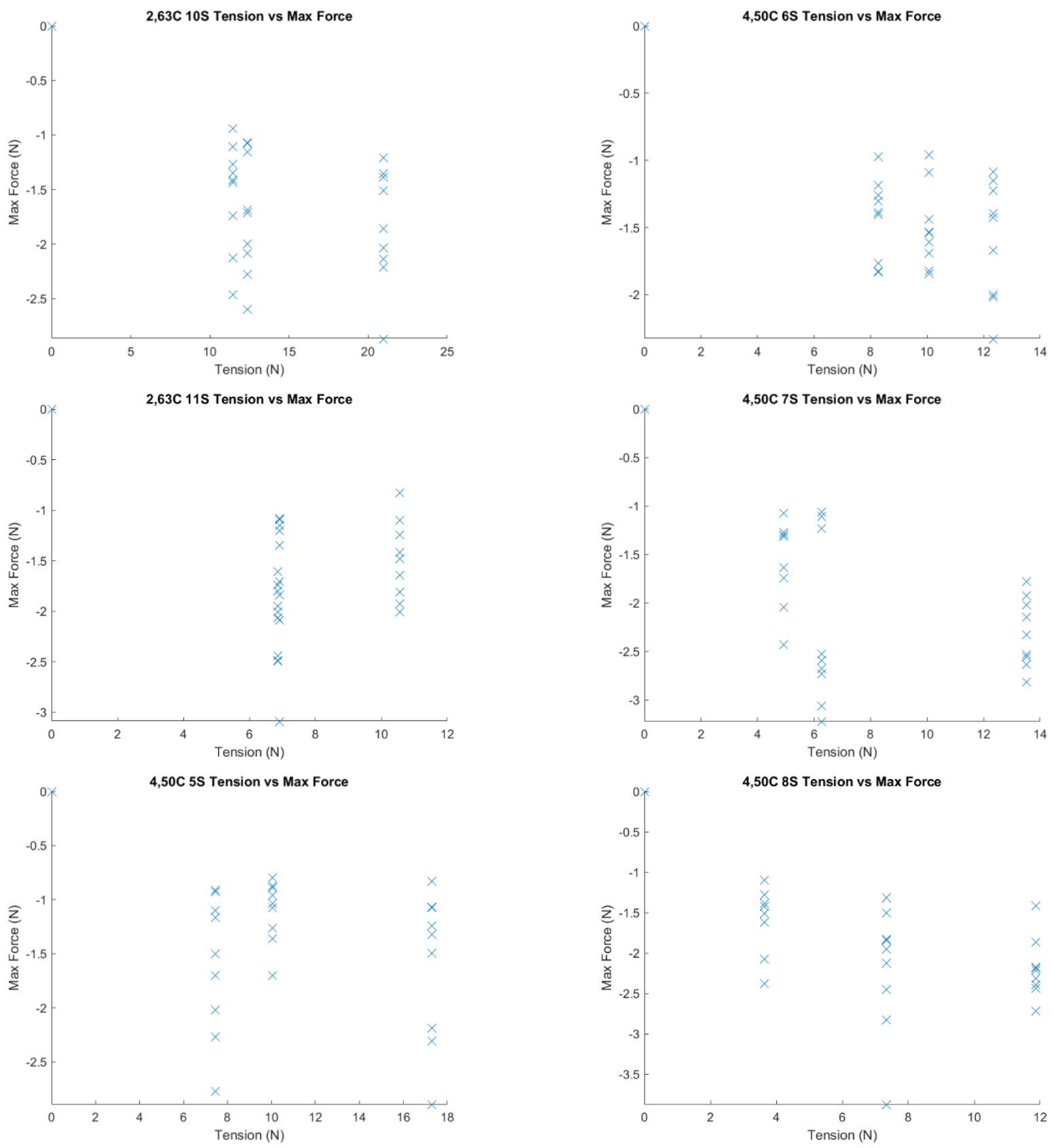

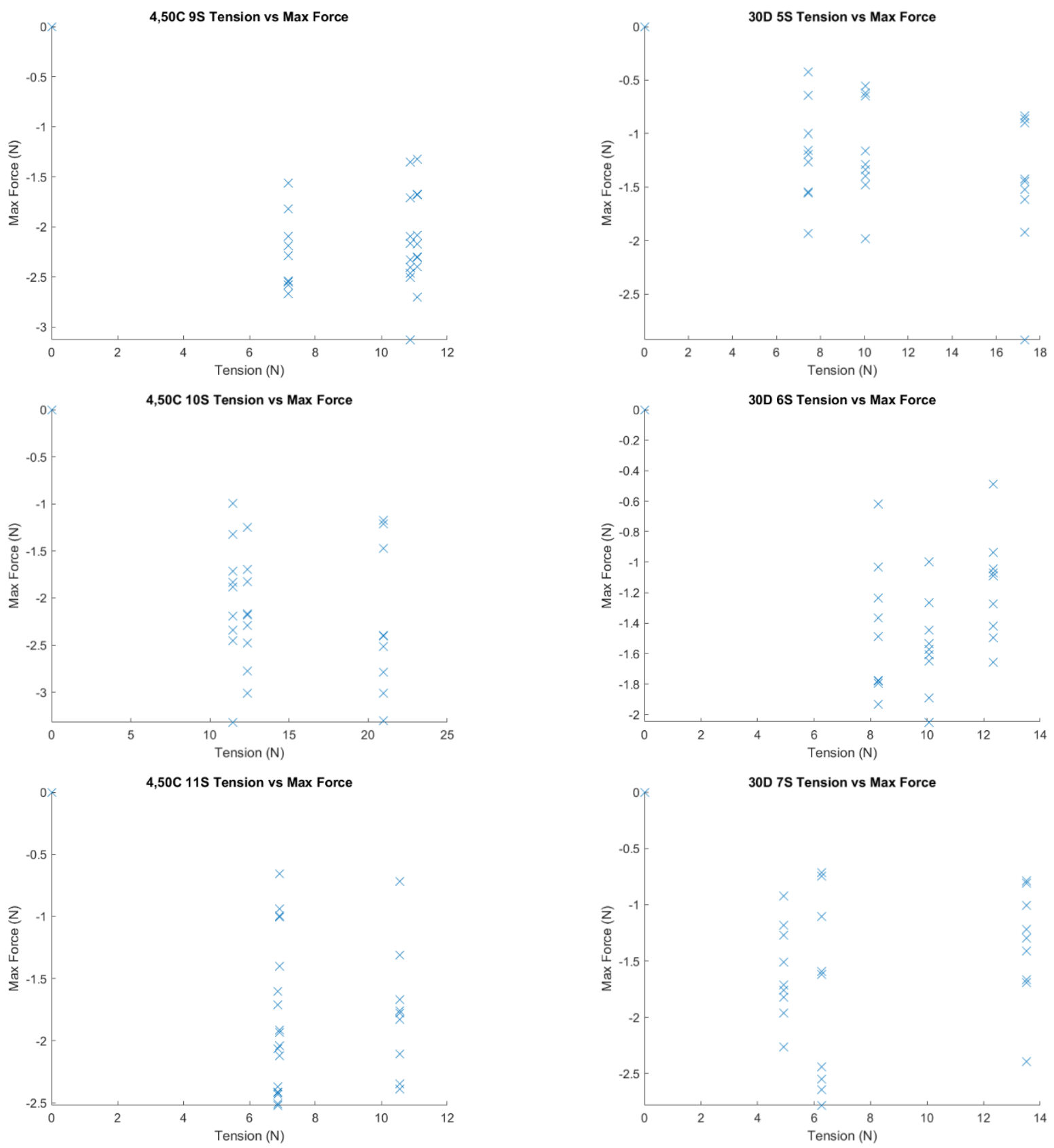

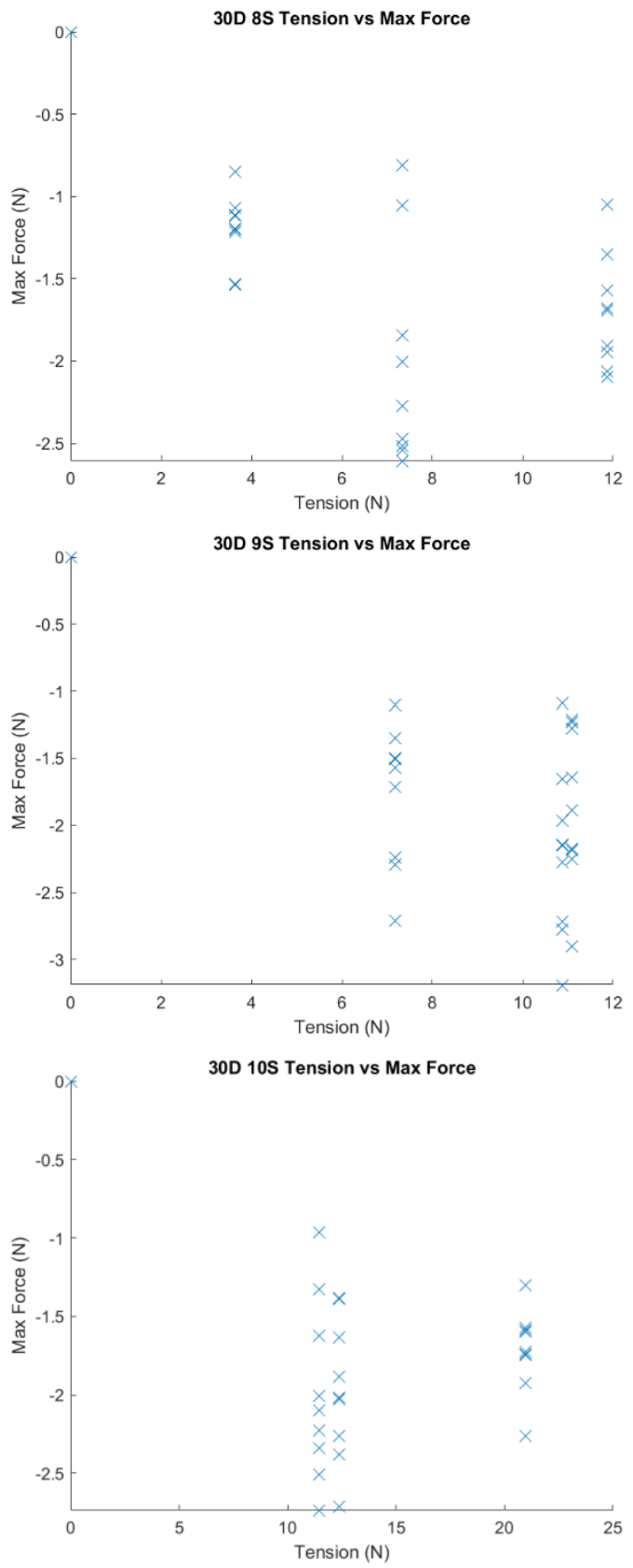
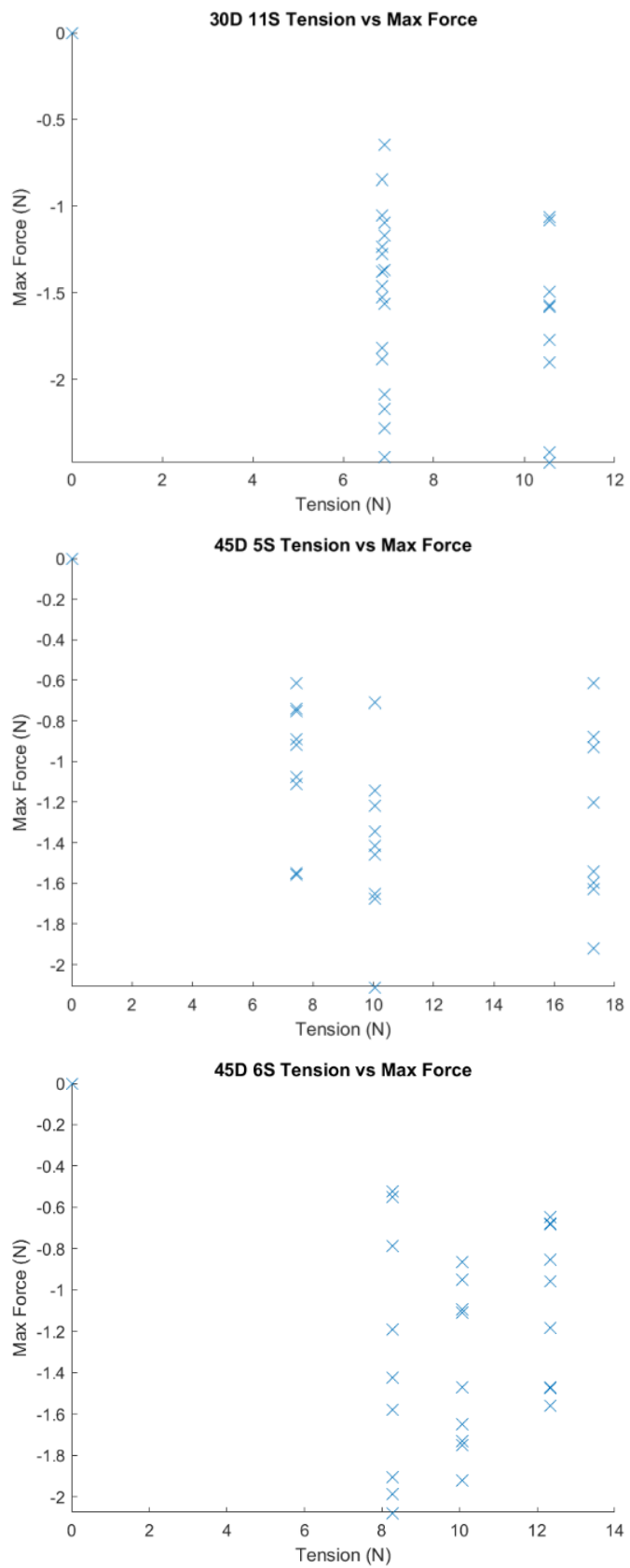

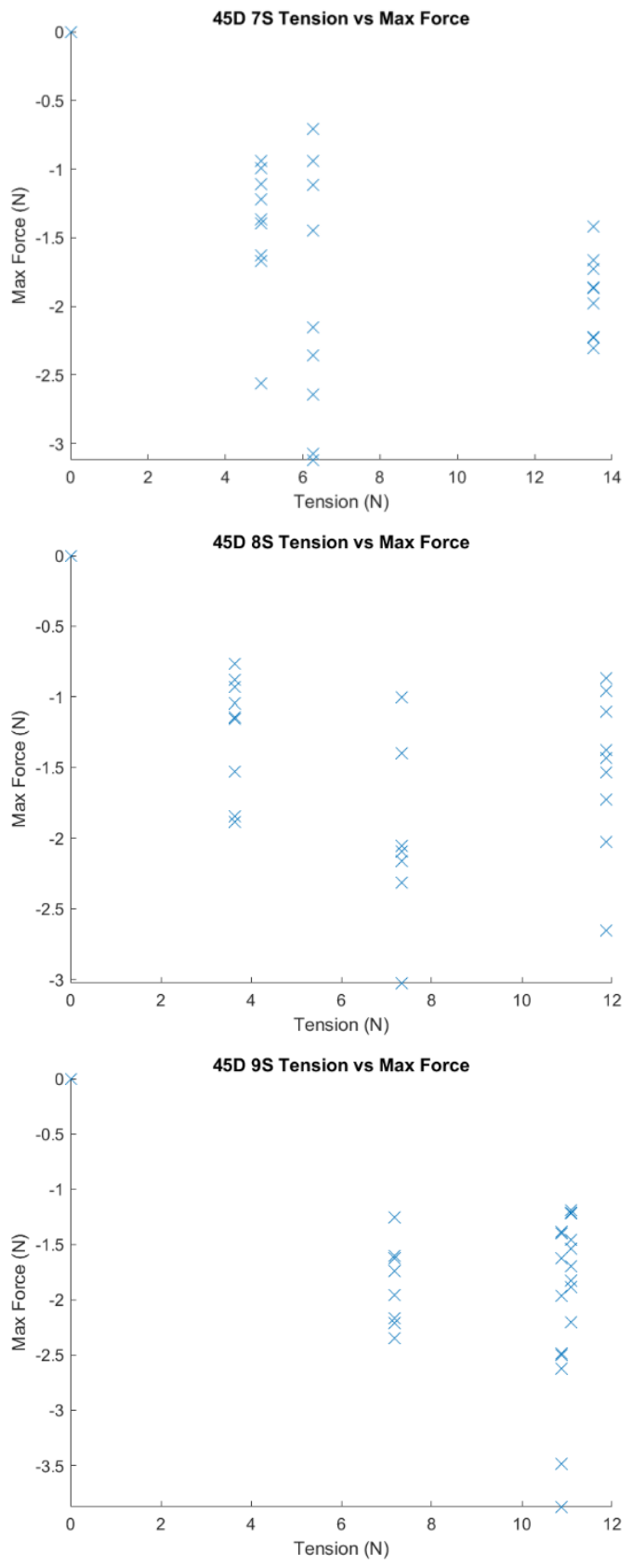
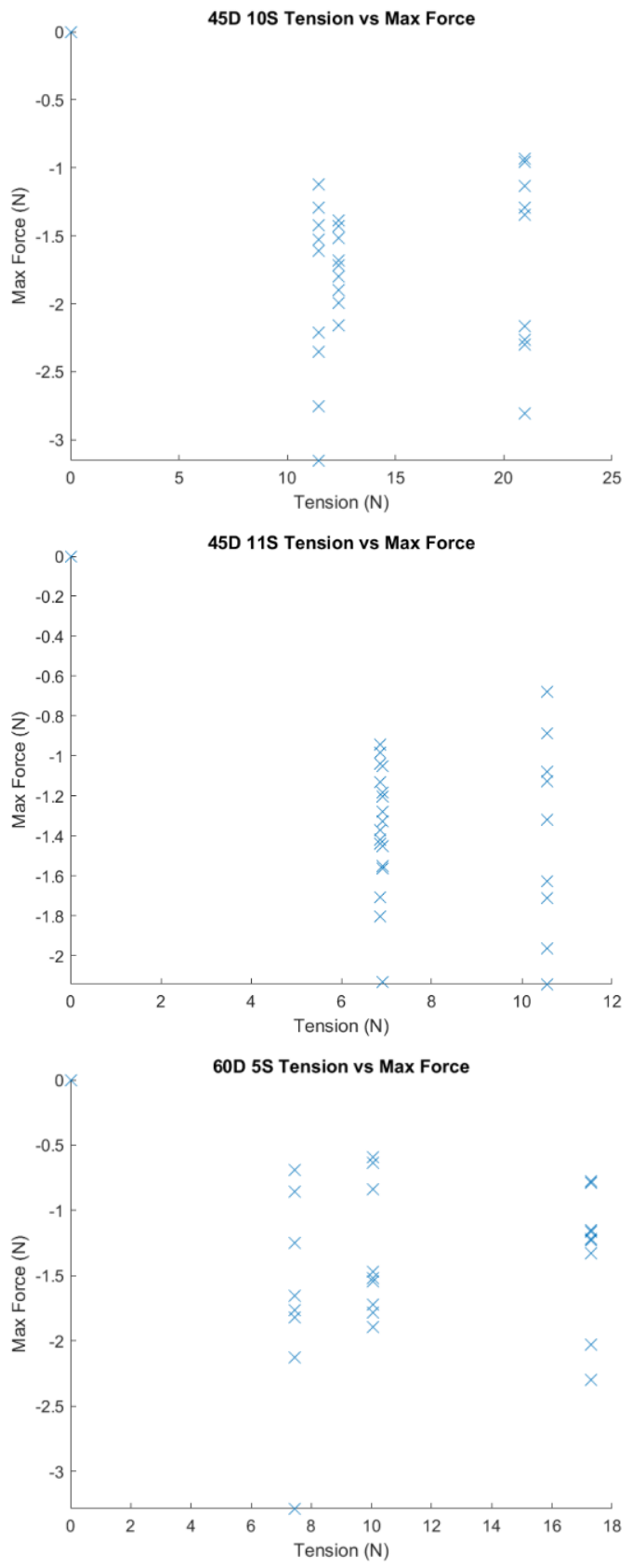

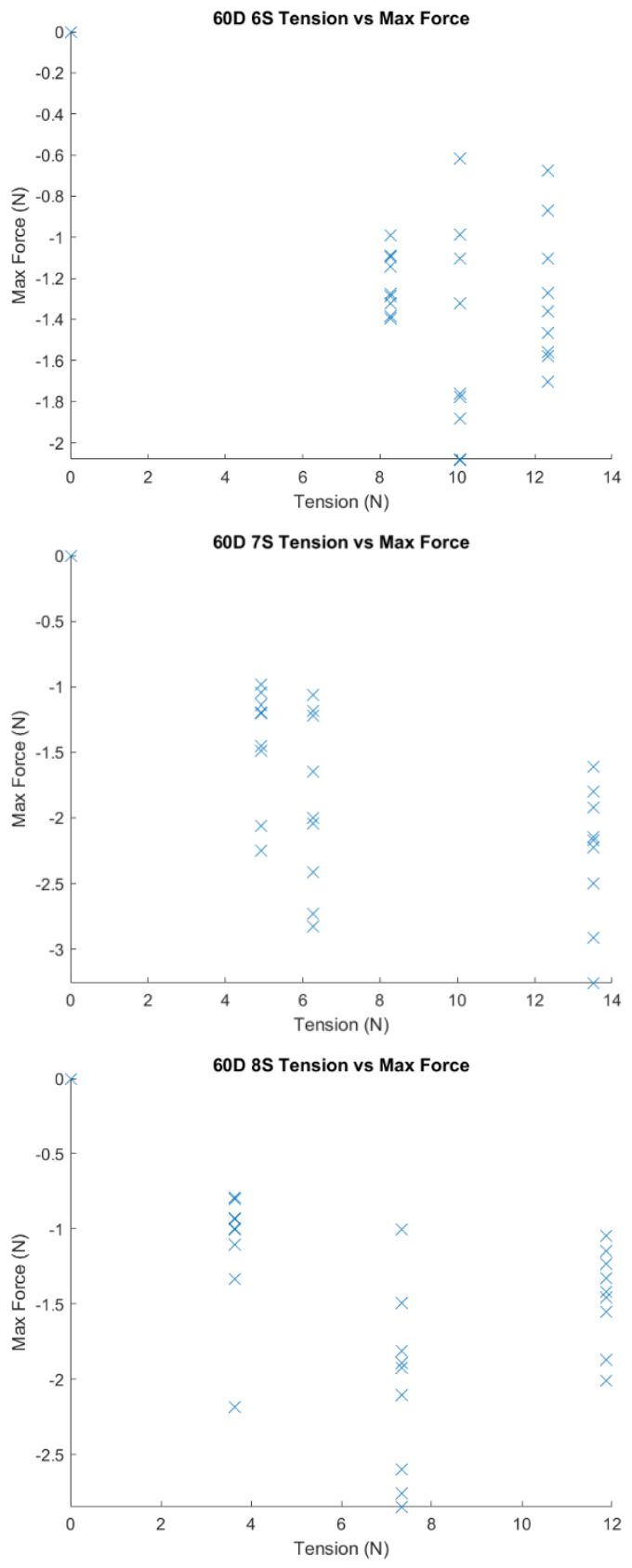
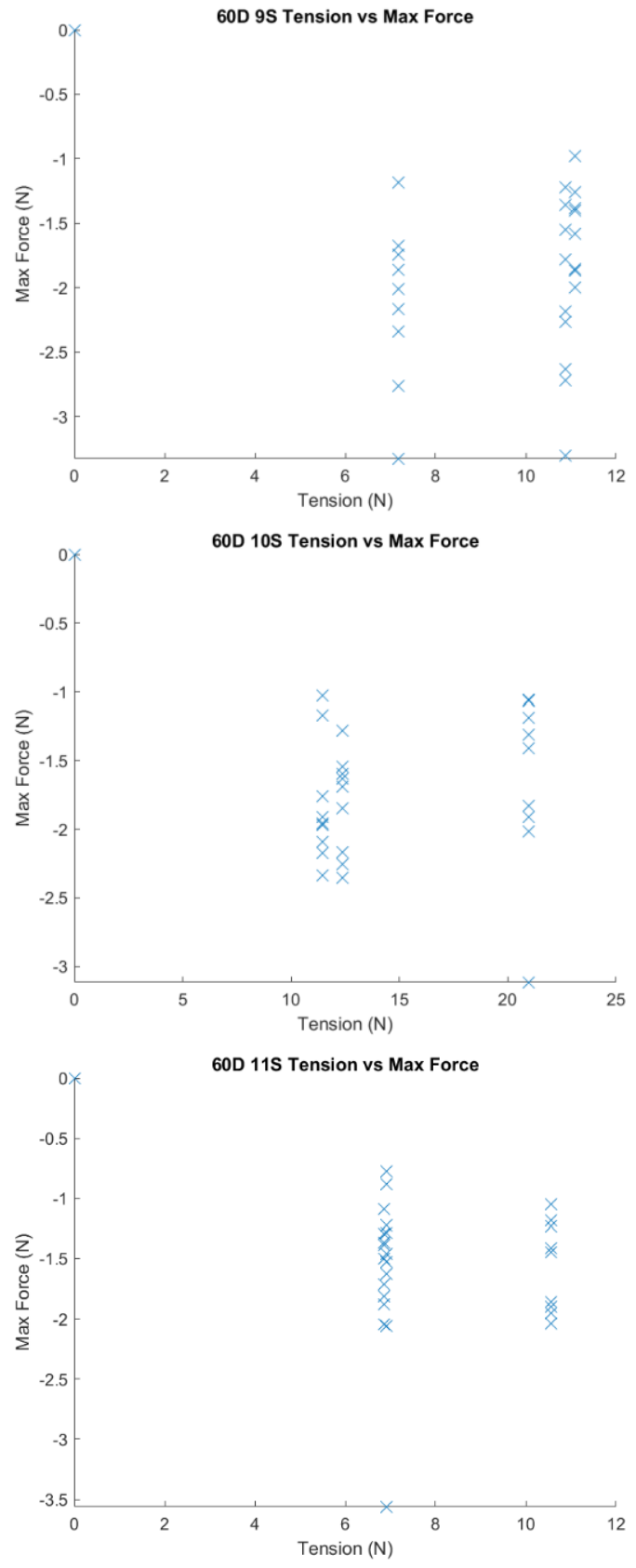

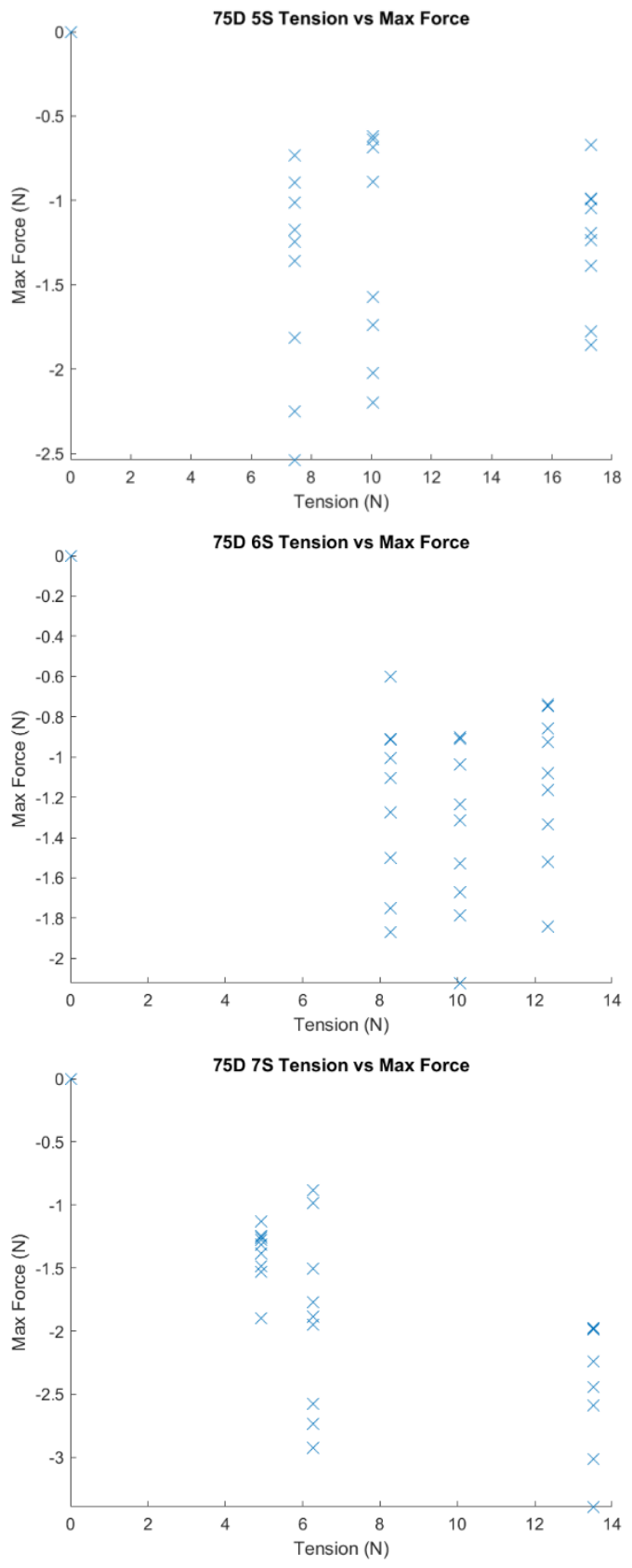
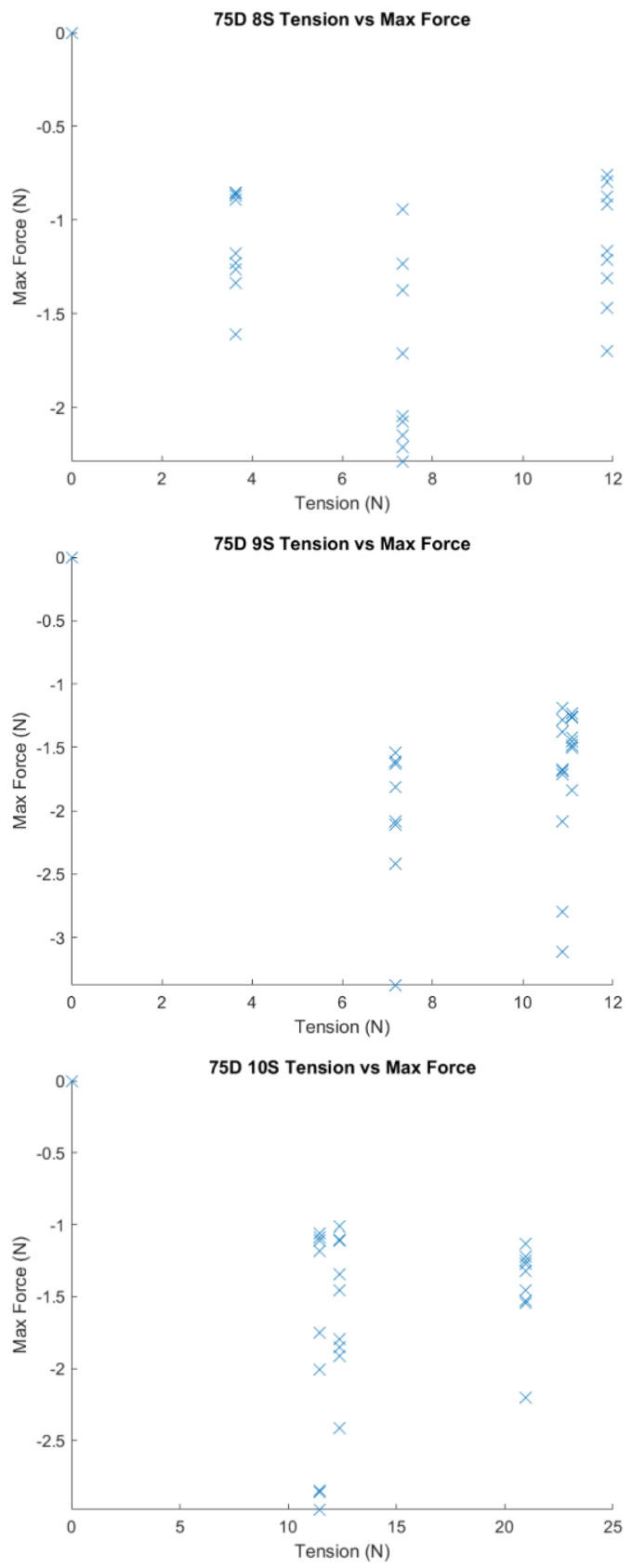

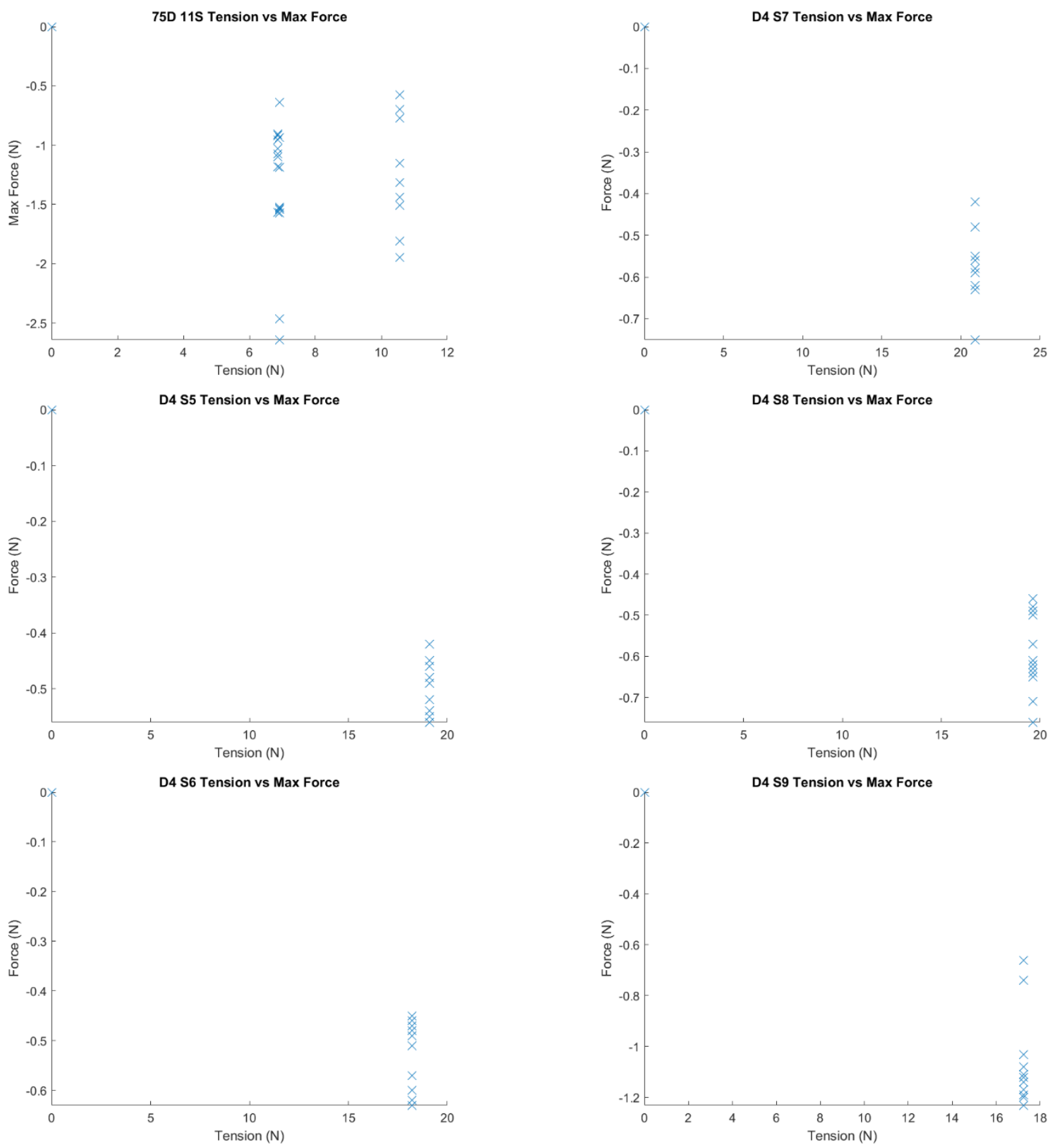

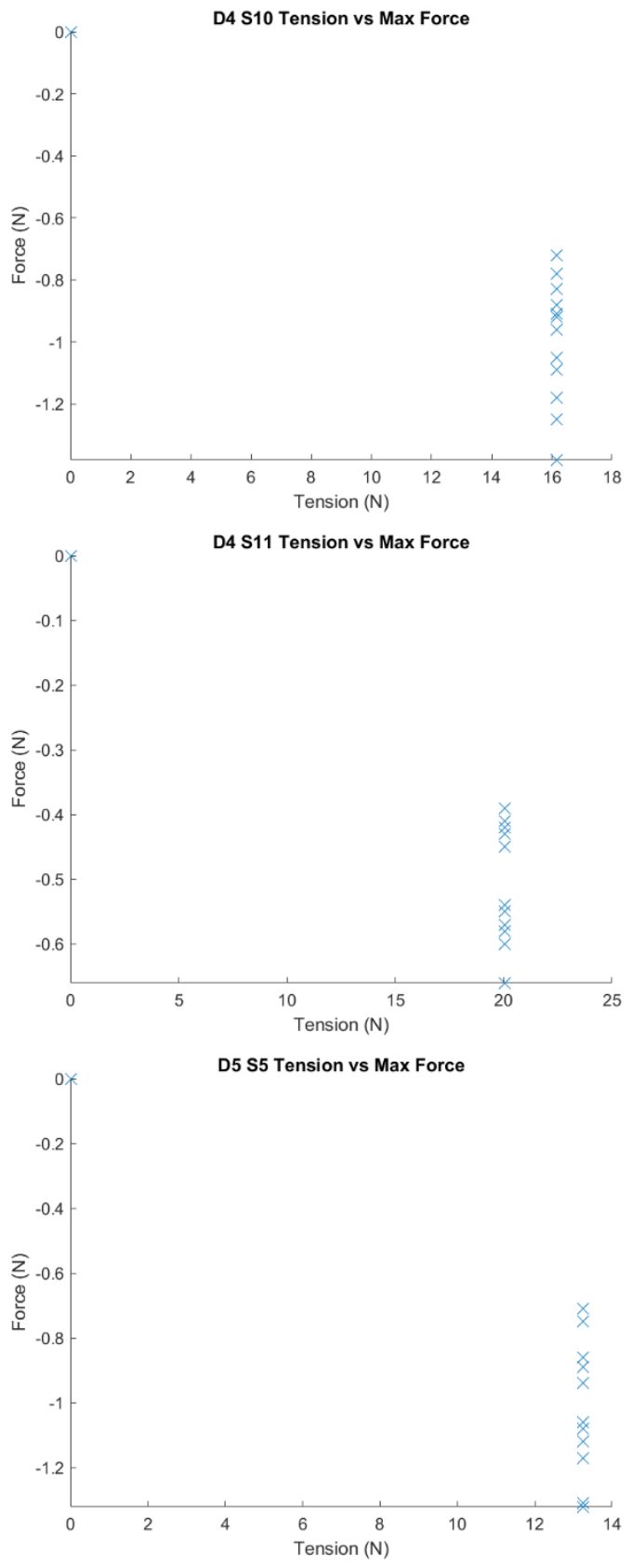
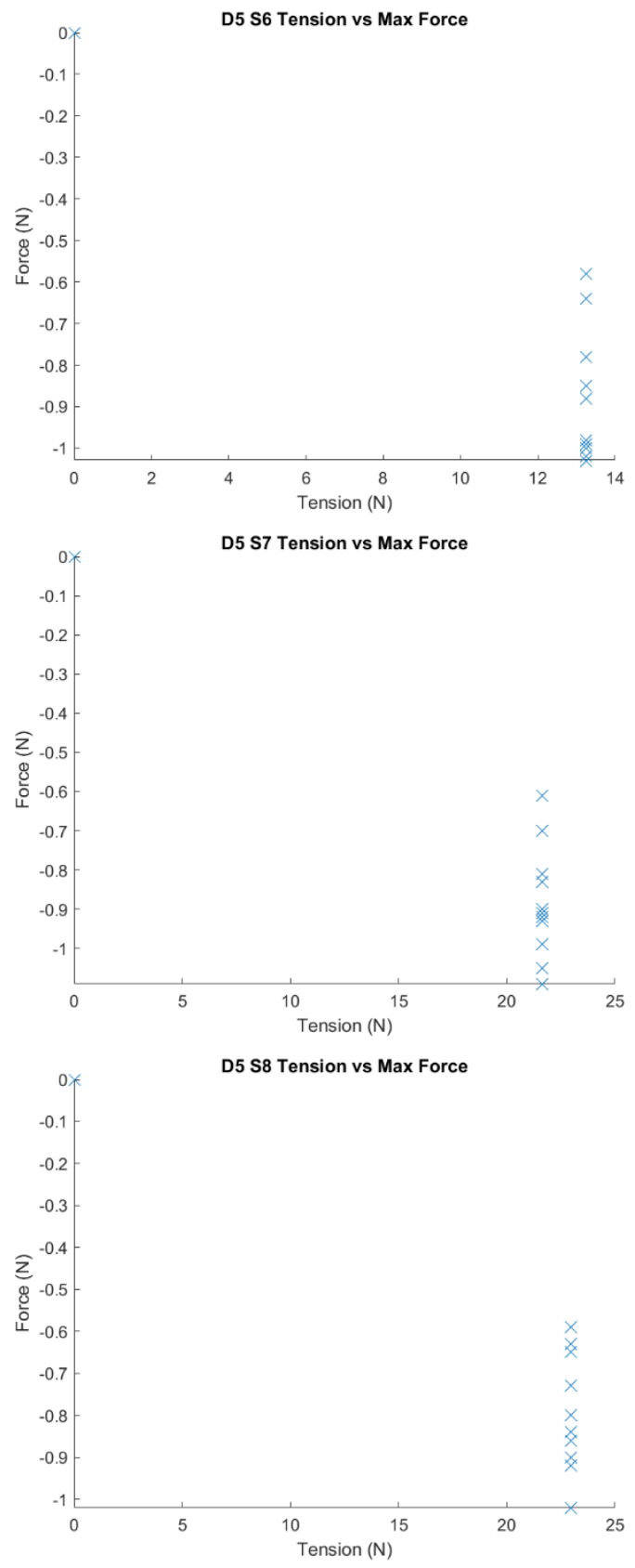

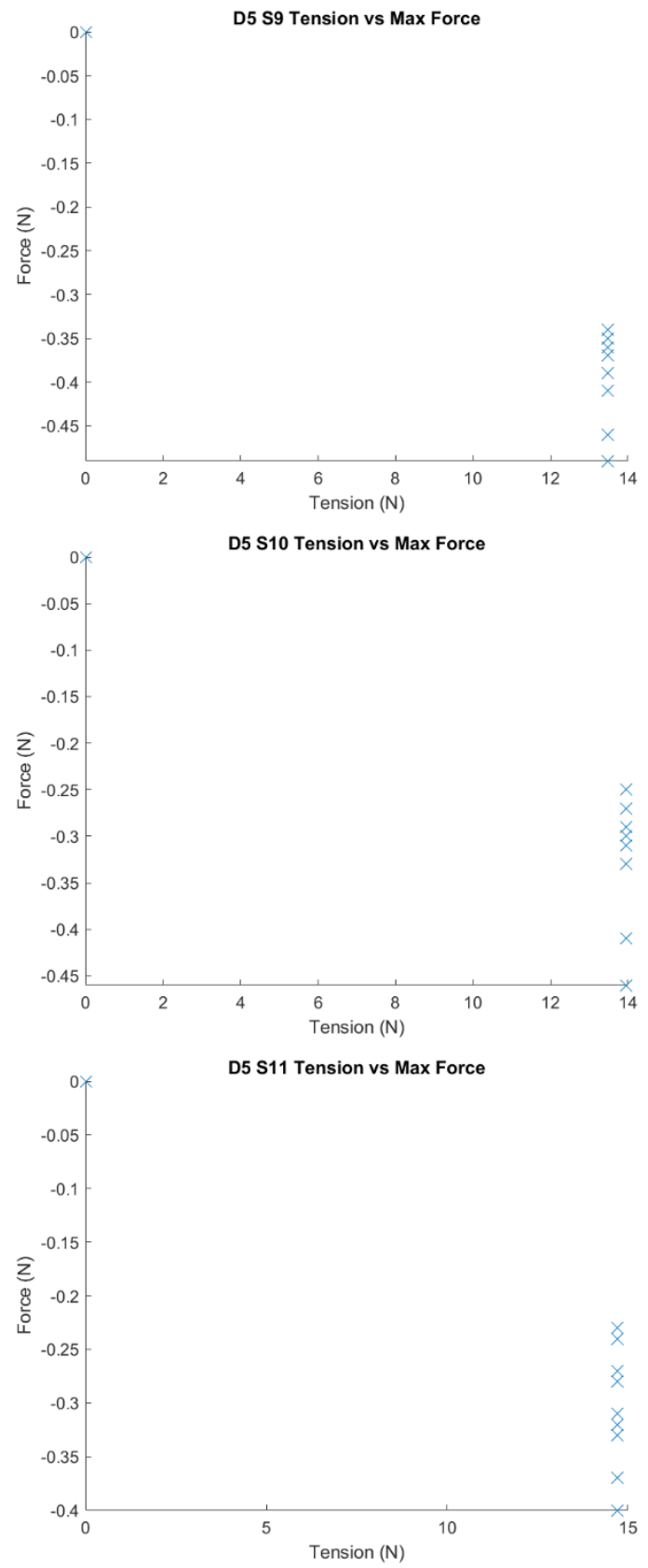
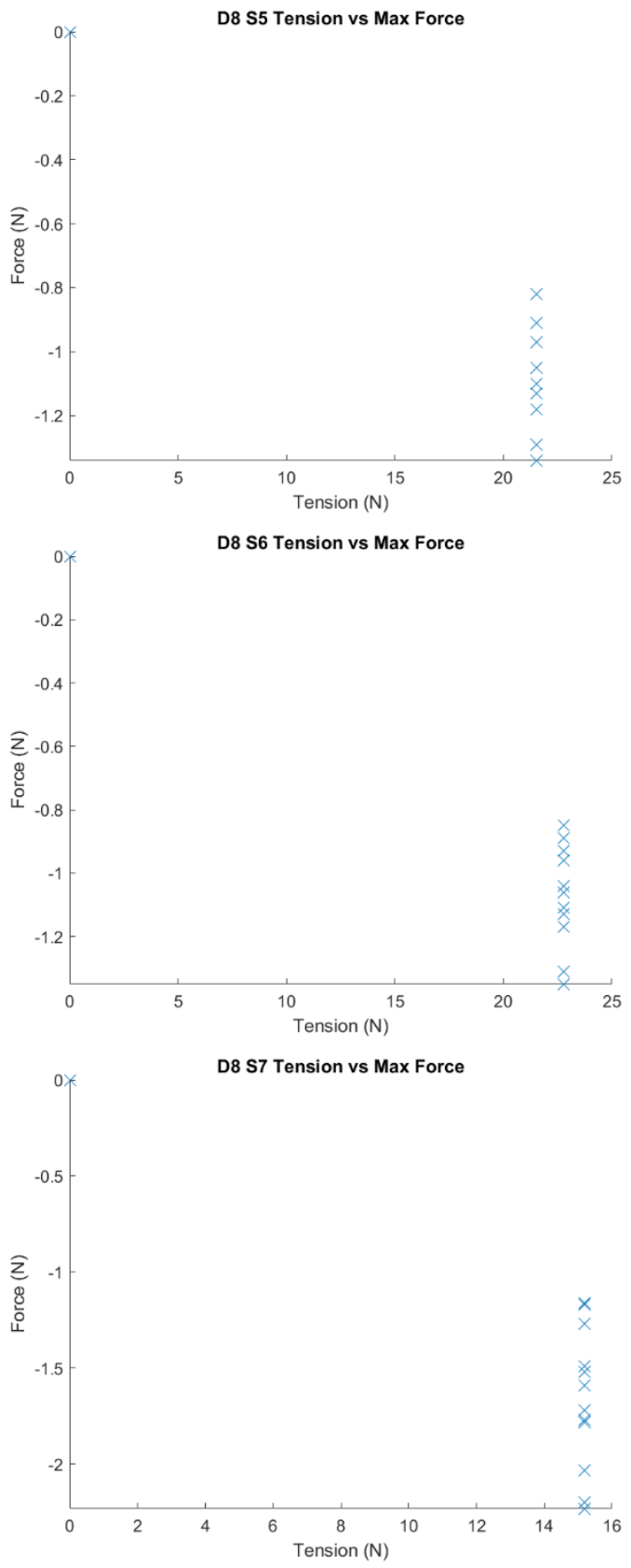

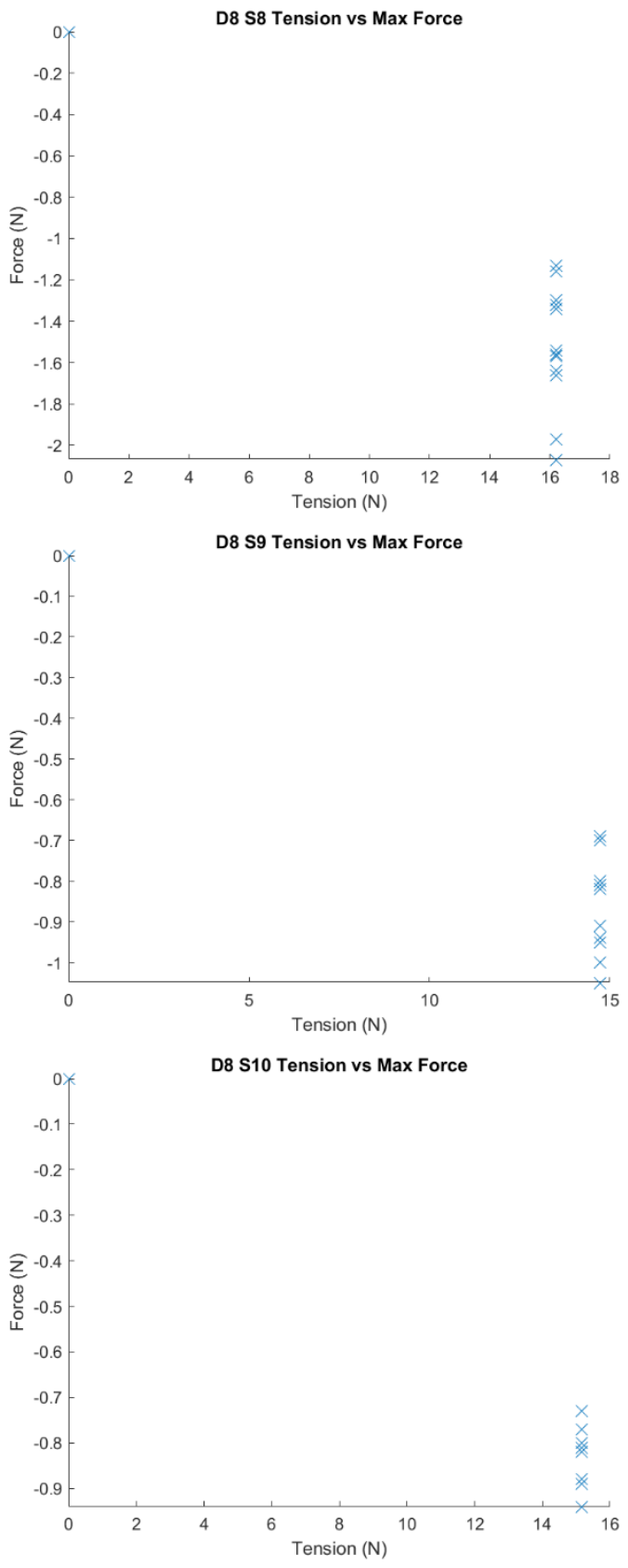
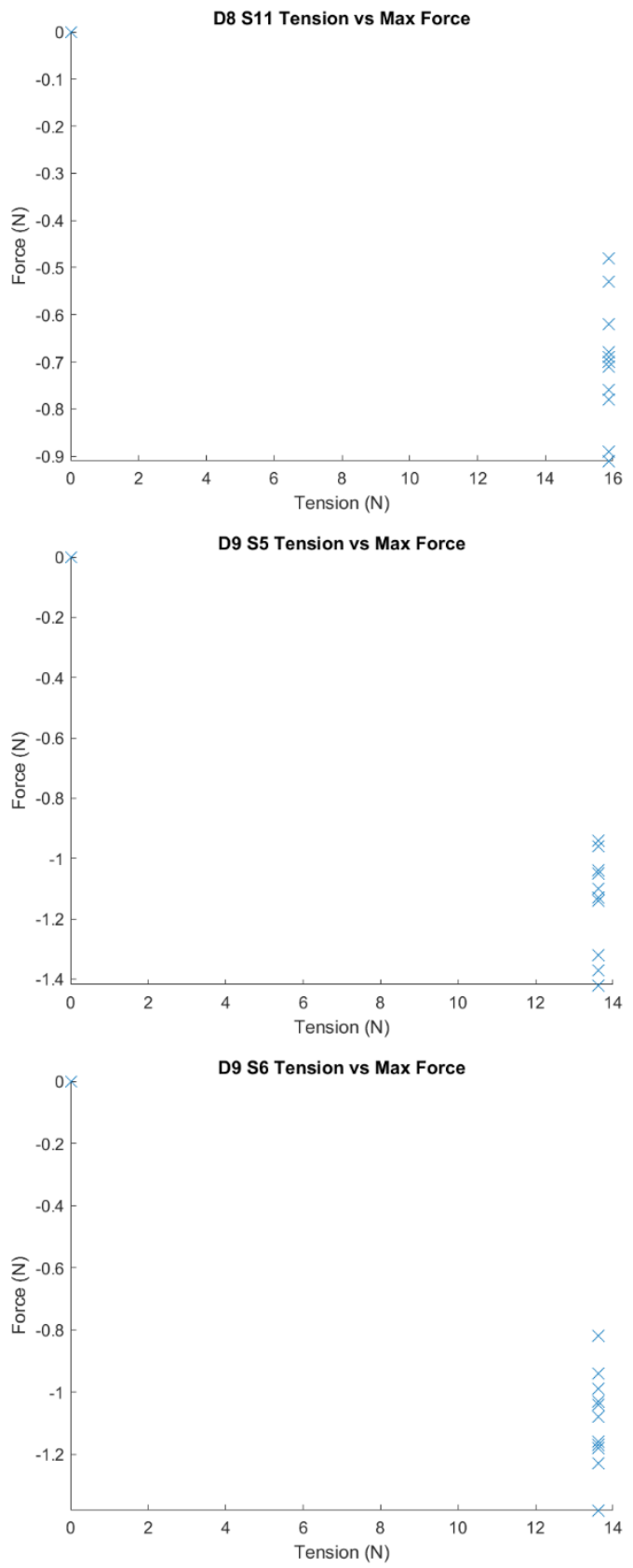

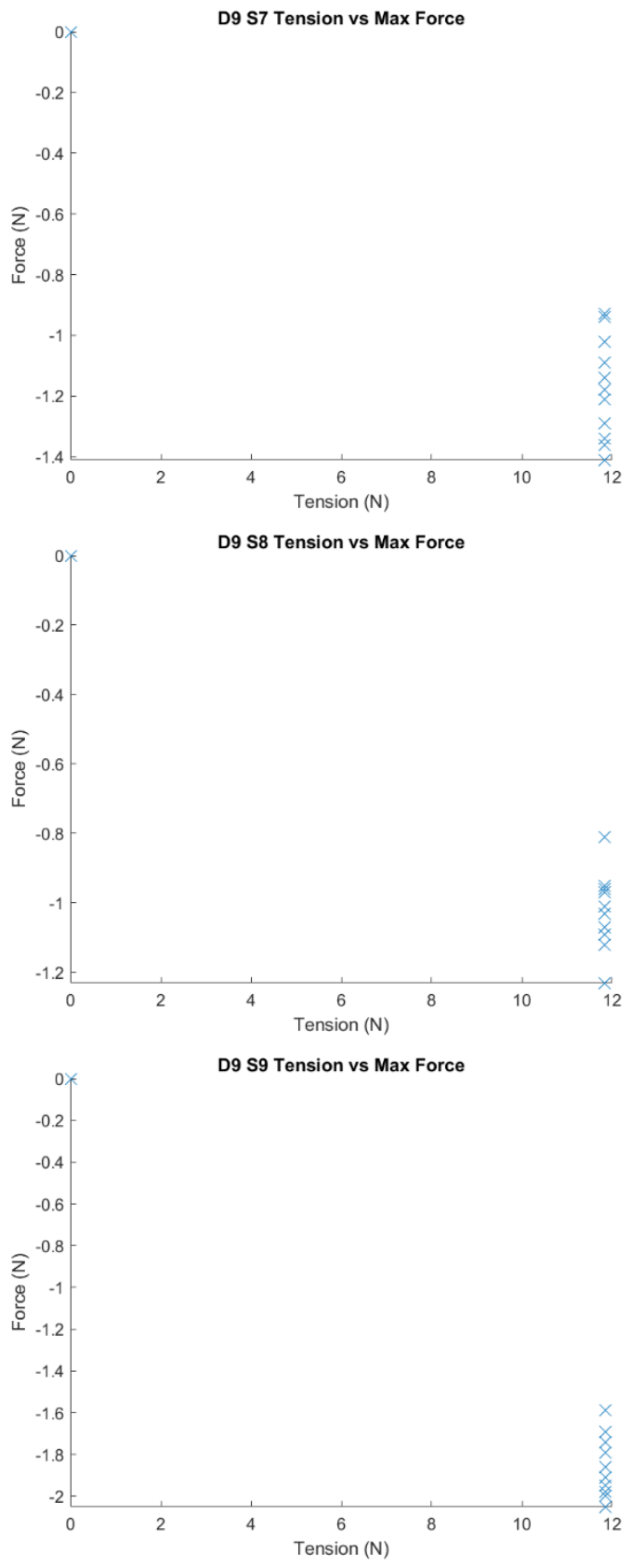
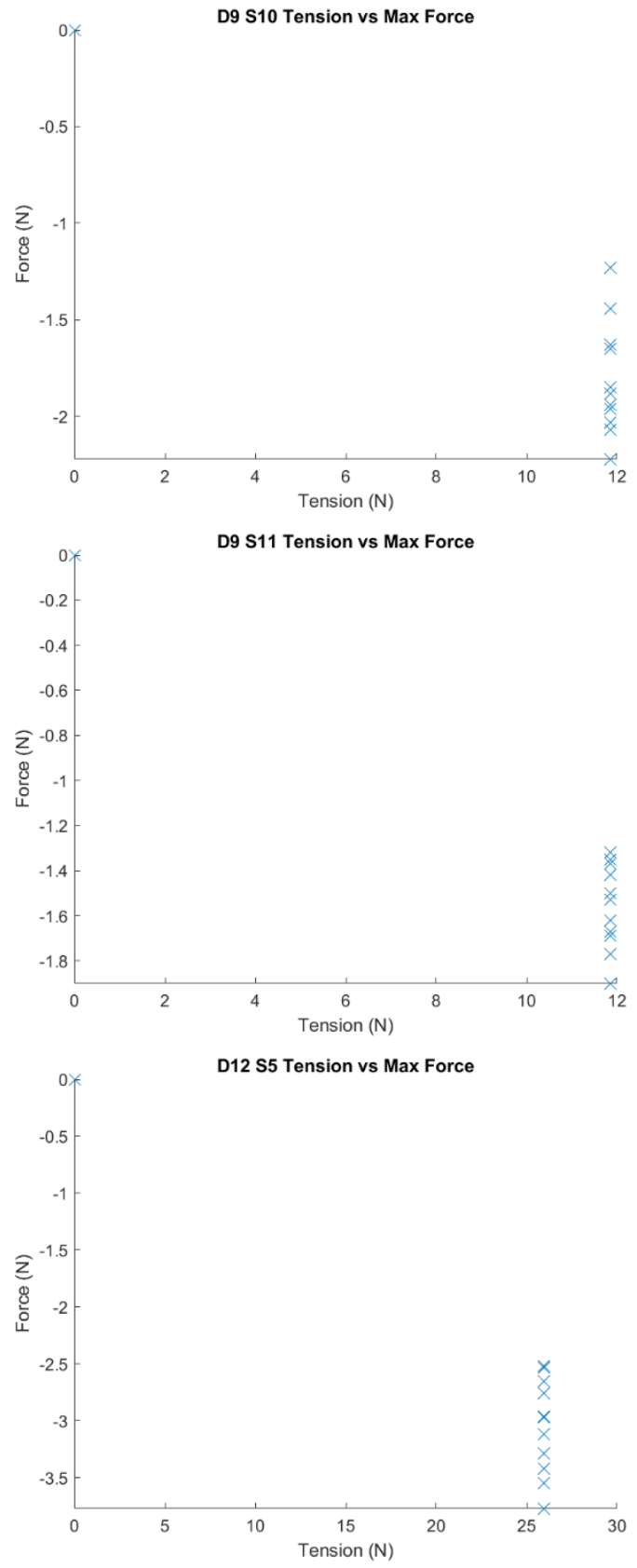

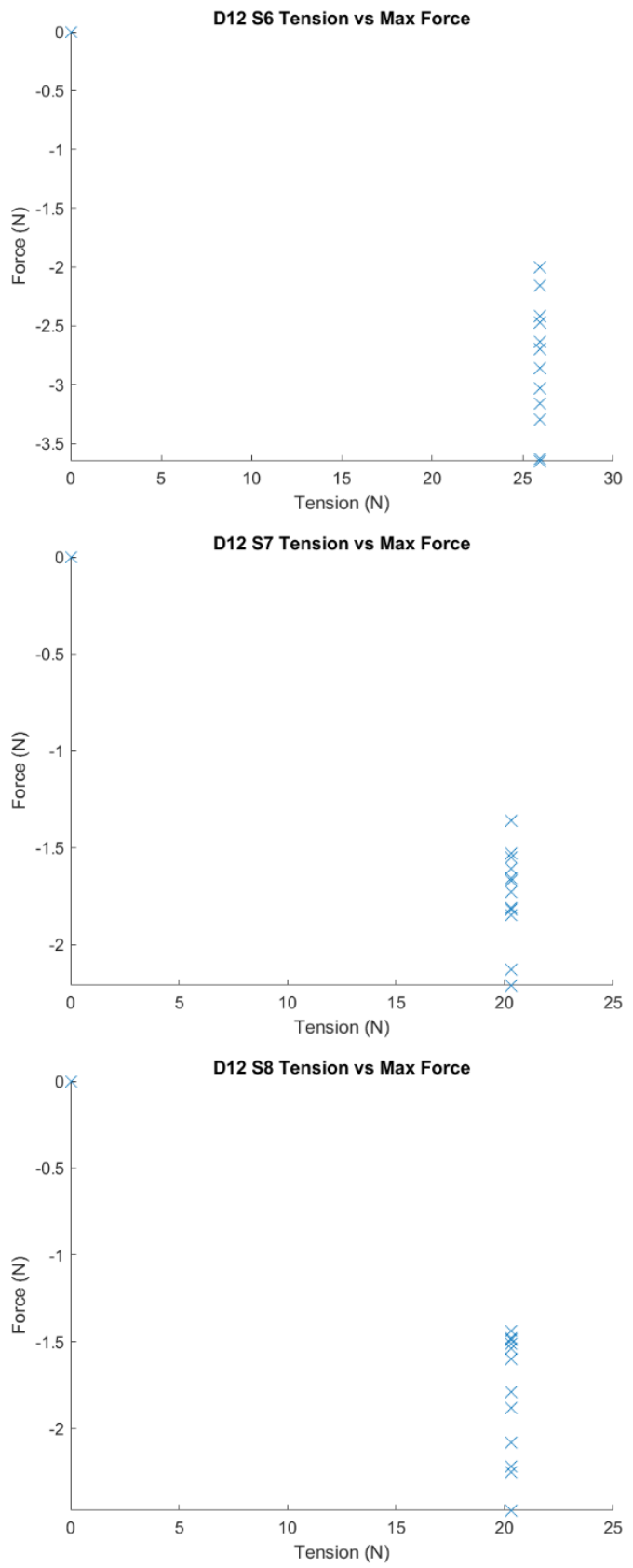
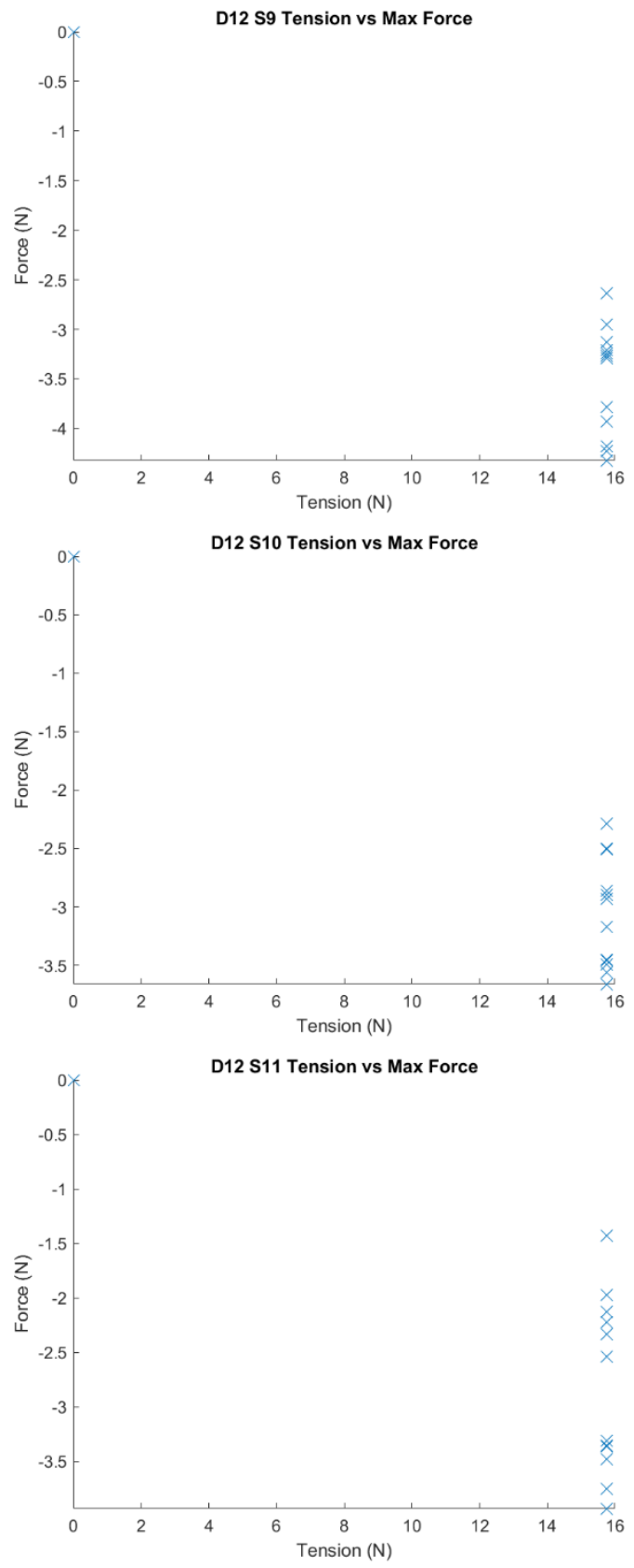


\section{Appendix B - Equipment List and Drawings}

This appendix gives a listing of all major materials used during experimentation and drawings of the experimental apparatus.

\section{Equipment list:}

- $\quad$ Arduino Due
- $\quad$ Omega Engineering - LCMFL-20N Inline Load Cell SN: 398537
- $\quad$ Interface SM-25-1 SN: 672365
- $\quad$ Interface SM-25-2 SN: 1010237
- $\quad$ Humimic Medical Ballistics gel \#0
- $\quad$ Actuonix LAC Board
- $\quad$ Sustom Built Test Frame
- $\quad$ Solatron S Series LDVT with BICM signal Conditioner
- $\quad$ EIKON-30 Stainless Steel Tattoo Needle
- $\quad$ Becton, Dickson and Company PrecisionGlide hypodermis needle
OMEGA PTFE tubing




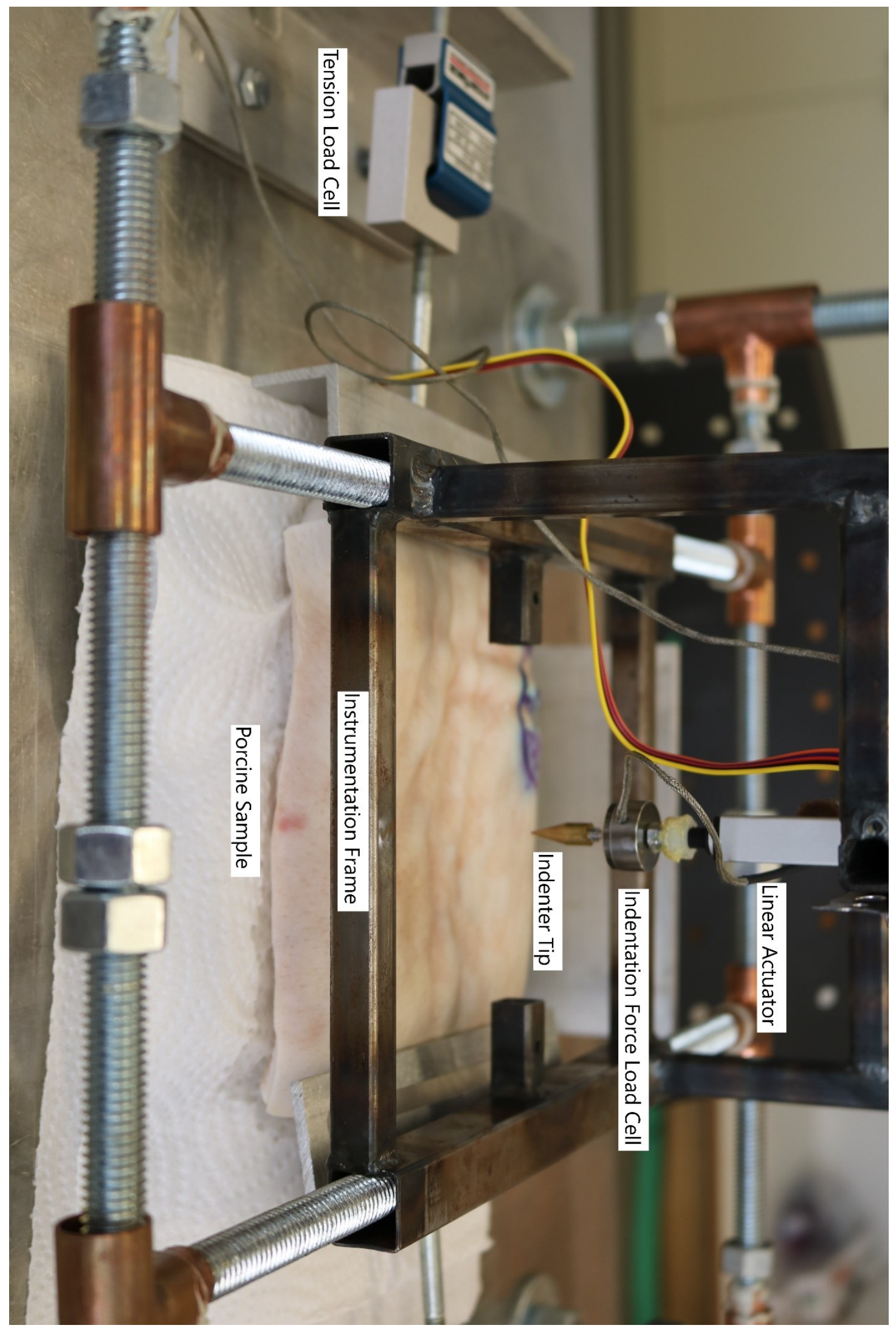




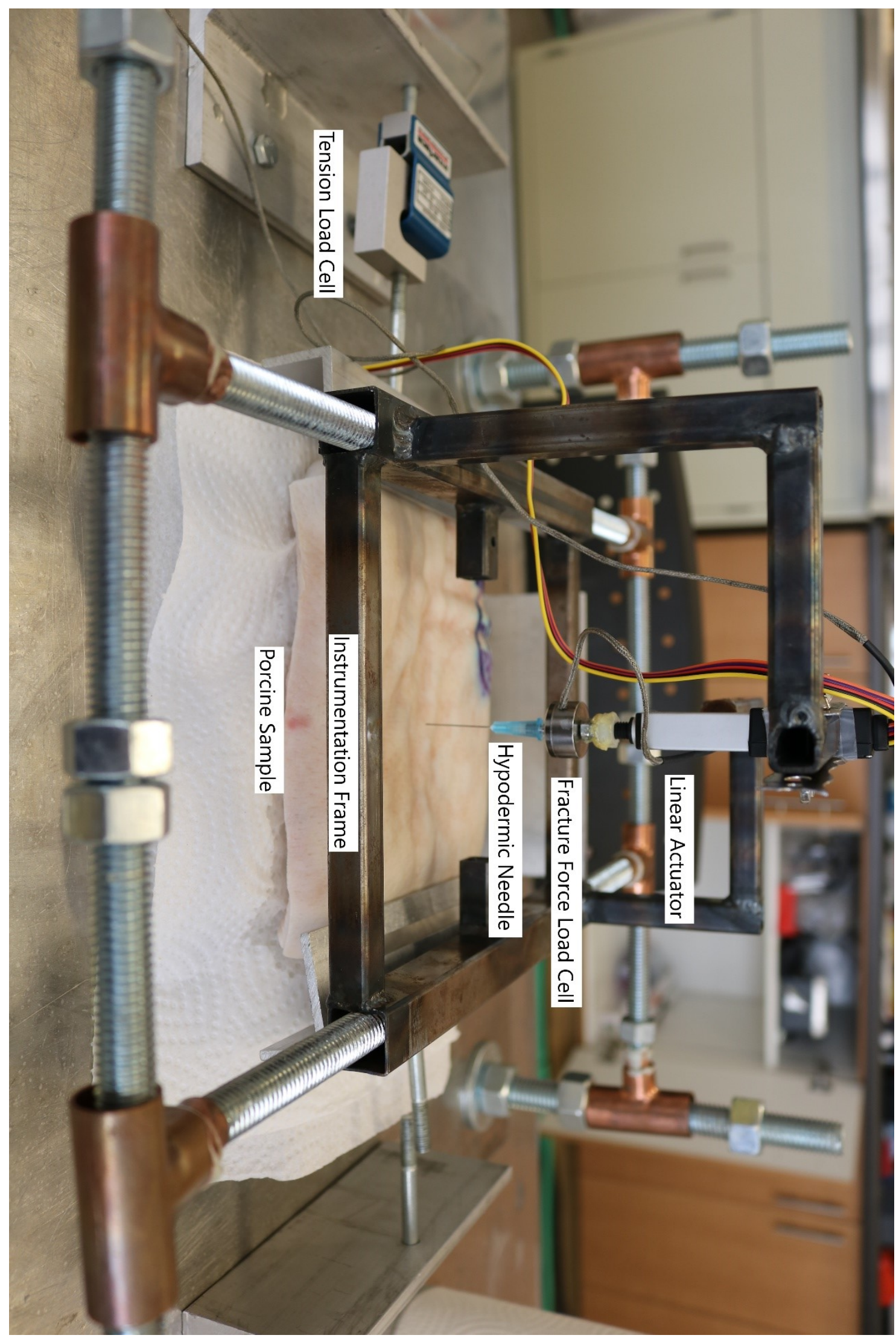



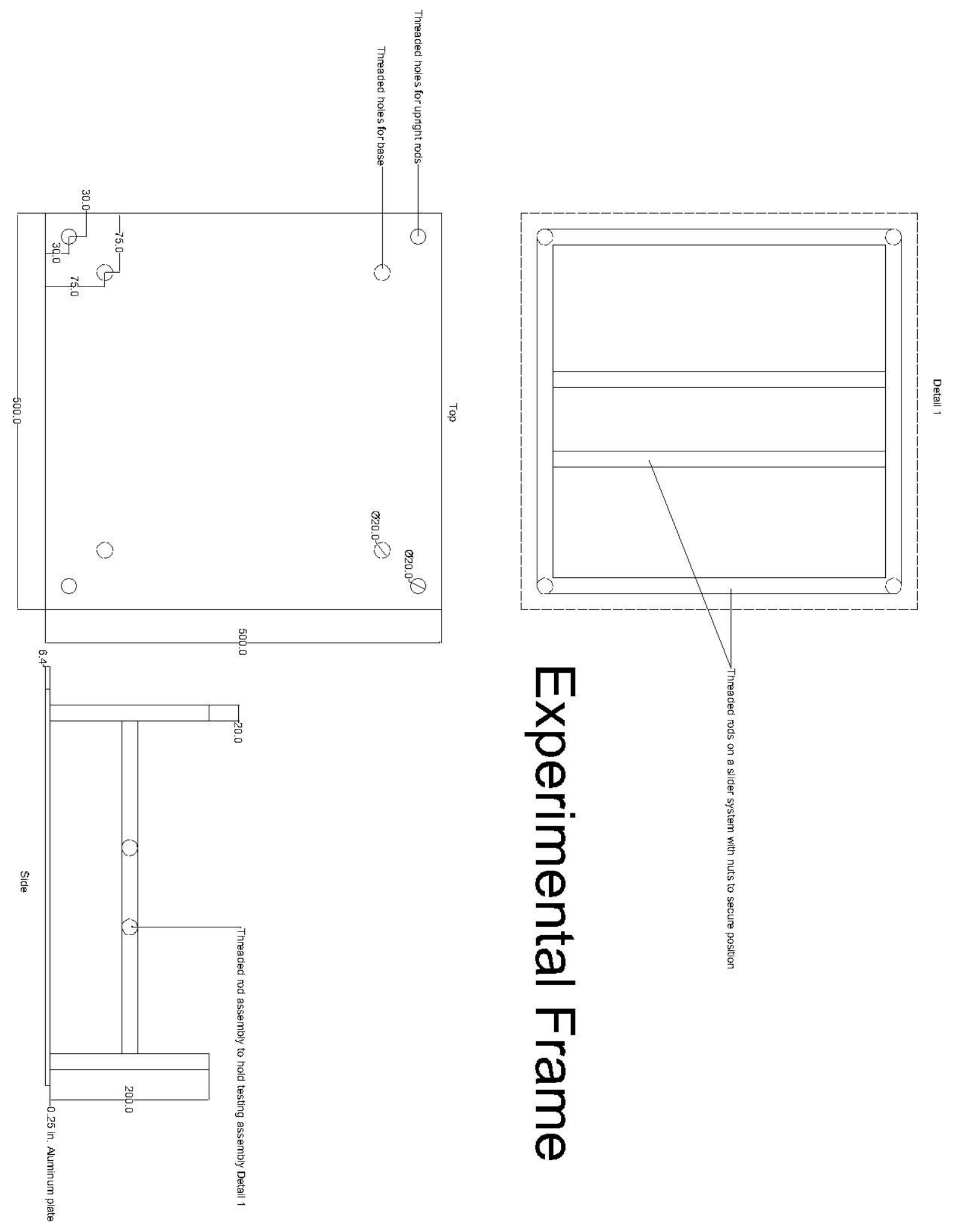


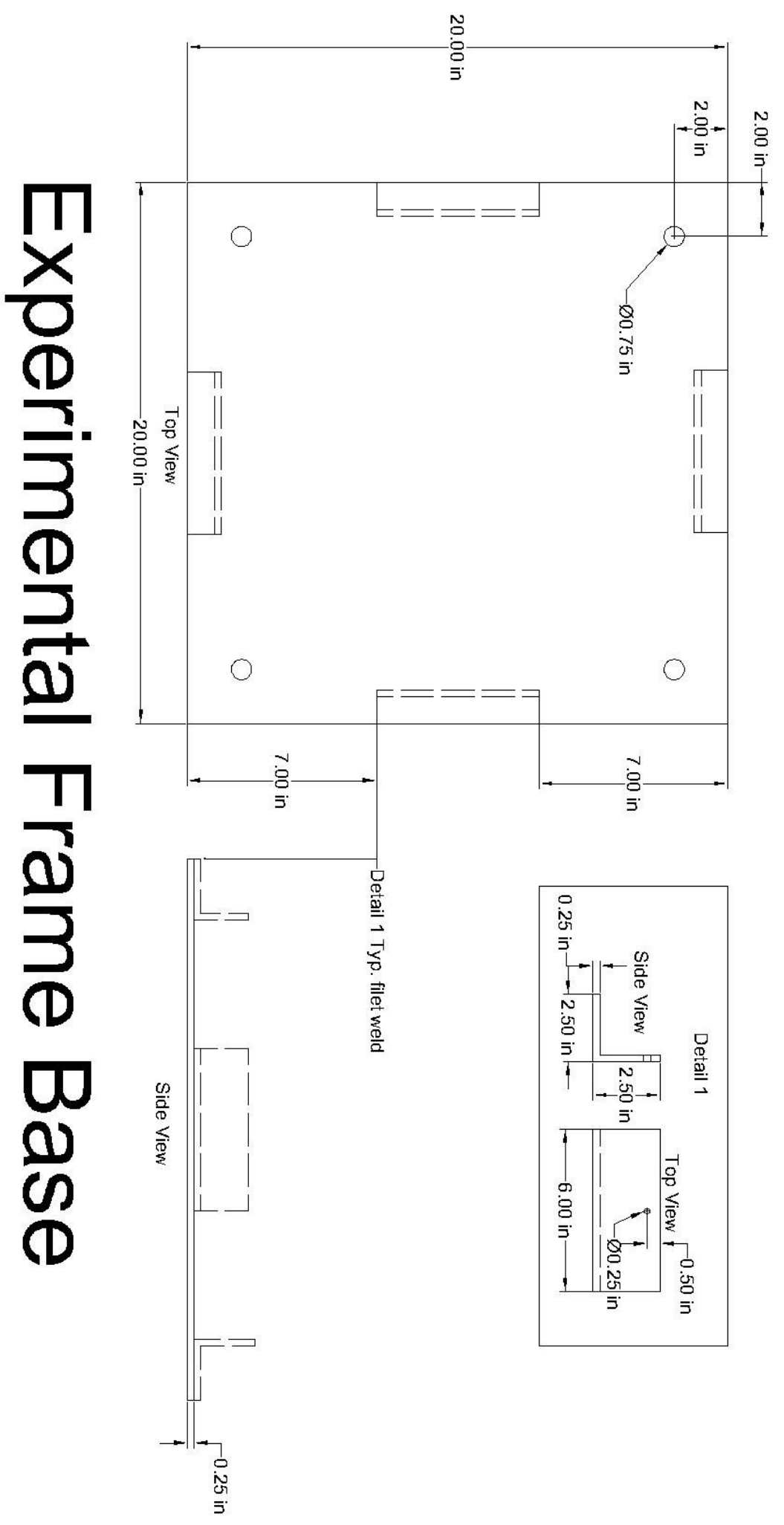




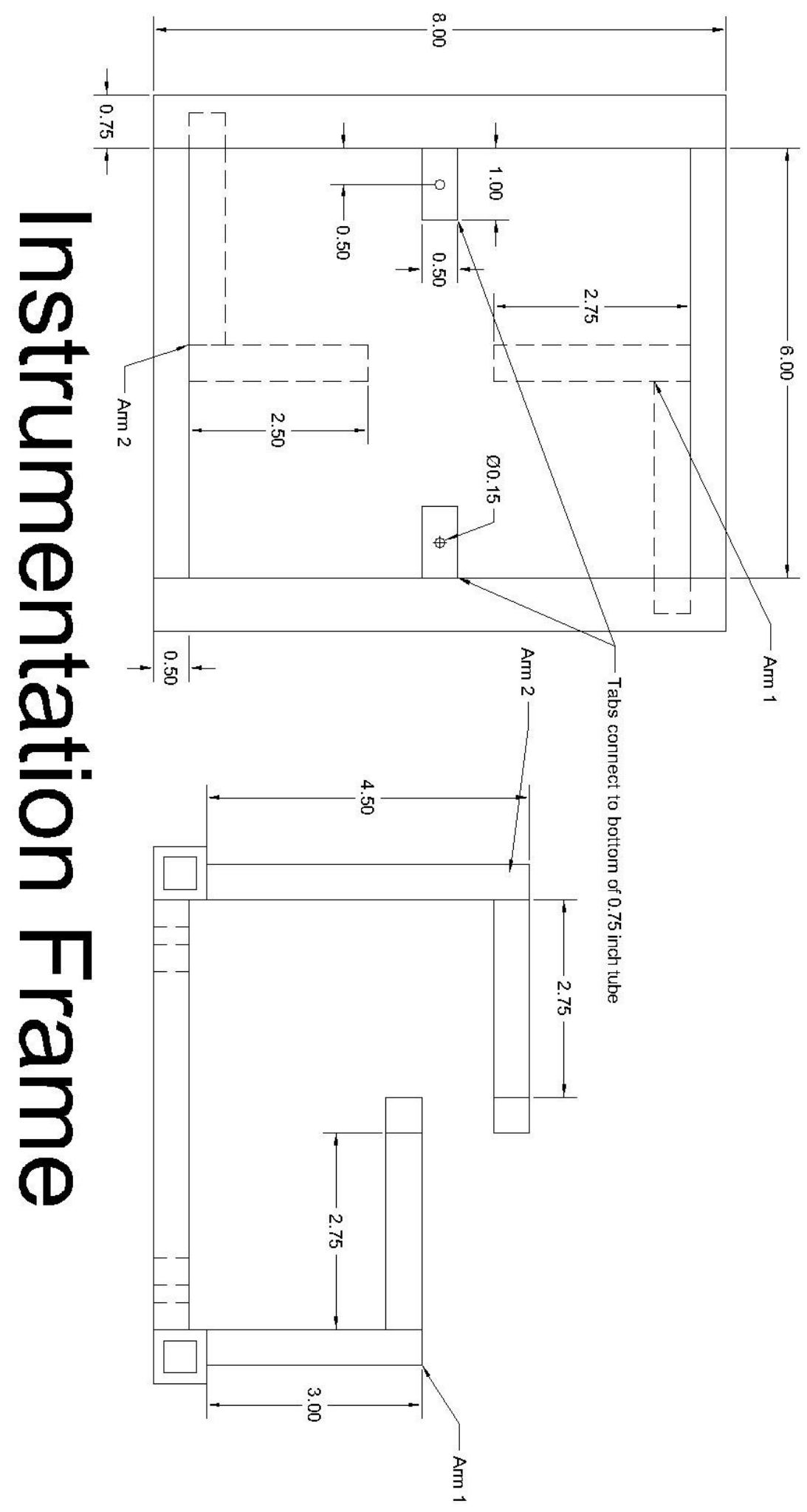




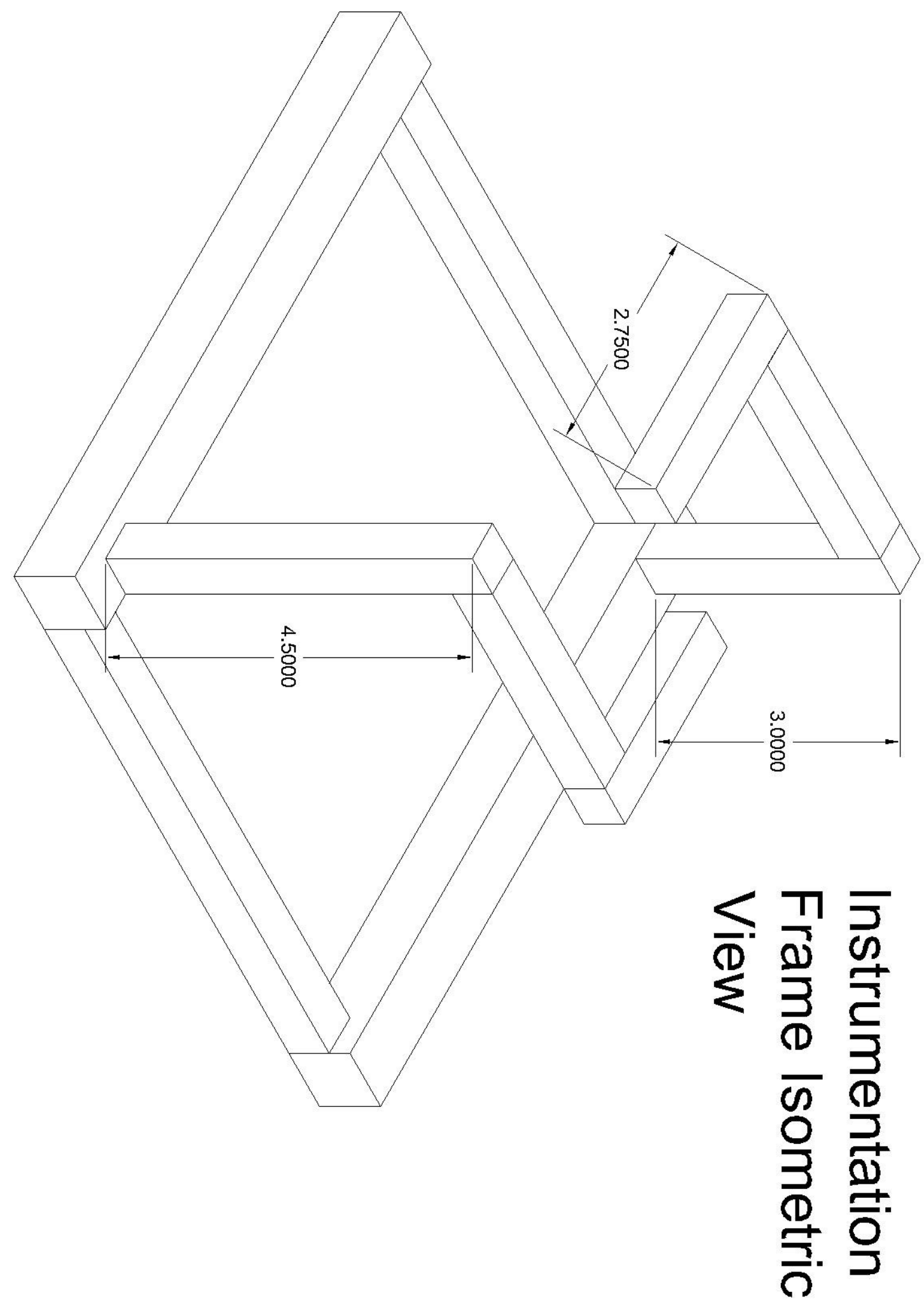



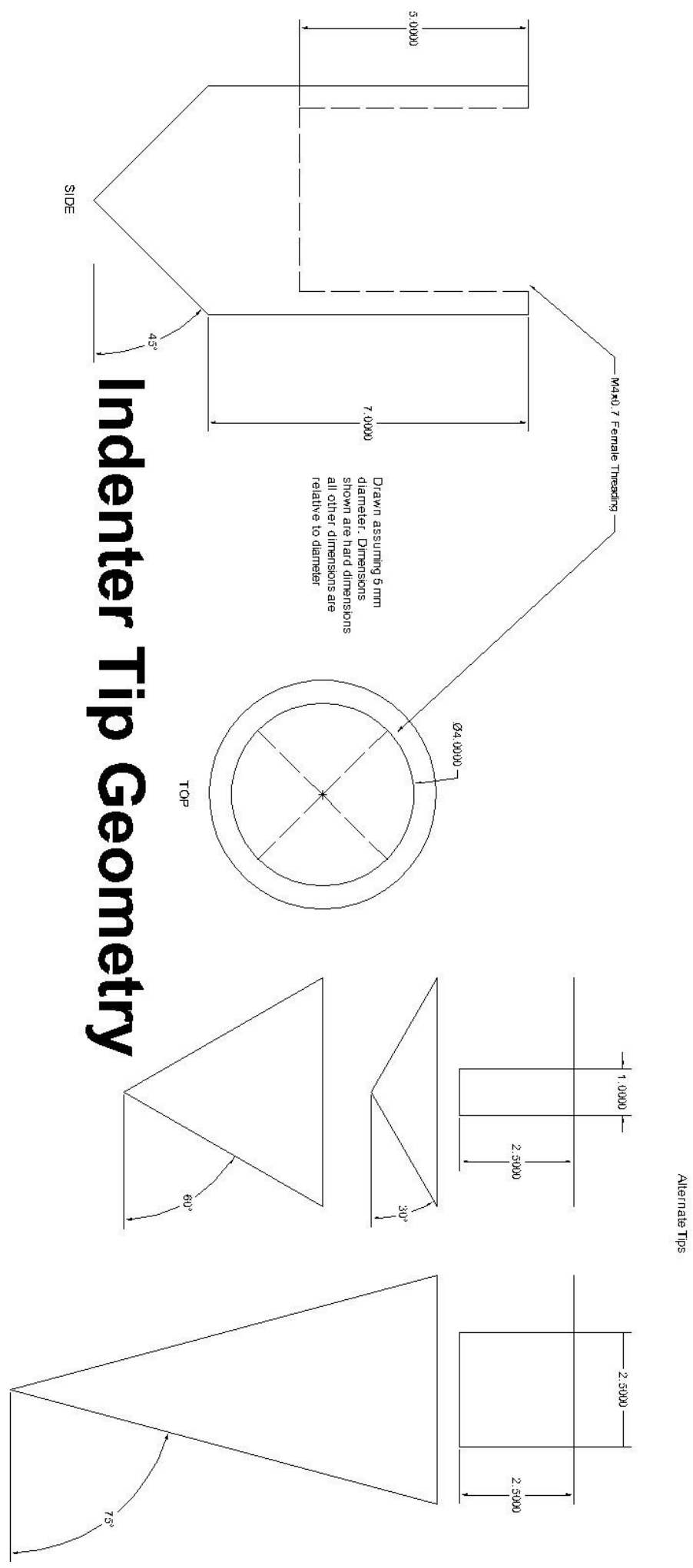


\section{Appendix C - Calibrations}

This appendix gives the calibration data of all instrumentation used in the experiments.

LCMFL-20N Serial Number: 398537

\begin{tabular}{|c|c|c|c|}
\hline Scale Accuracy & $0.05 \mathrm{~g}$ & & \\
\hline HX711 Amp \# & 1 & & \\
\hline $\begin{array}{l}\text { Amp Calibration } \\
\text { Factor }\end{array}$ & -156000 & & \\
\hline Load Cell Zero & $\sim 0.4 \mathrm{~N}$ & $\begin{array}{l}\text { Load Cell } \\
\text { Accuracy }\end{array}$ & $\sim 0.1 \mathrm{~N}$ \\
\hline \multicolumn{4}{|l|}{ Tension } \\
\hline Weight (g) & Load (N) & Reading (N) & Reading (g) \\
\hline 0.00 & 0 & 0 & 0.00 \\
\hline 10.40 & 0.10 & 0.10 & 10.19 \\
\hline 12.70 & 0.12 & 0.12 & 12.23 \\
\hline 34.00 & 0.33 & 0.34 & 34.66 \\
\hline 48.00 & 0.47 & 0.47 & 47.91 \\
\hline 70.65 & 0.69 & 0.69 & 70.34 \\
\hline 92.70 & 0.91 & 0.91 & 92.76 \\
\hline 115.35 & 1.13 & 1.14 & 116.21 \\
\hline 162.75 & 1.60 & 1.62 & 165.14 \\
\hline & & & \\
\hline \multicolumn{4}{|l|}{ Compression } \\
\hline Weight (g) & Load (N) & Reading (N) & Reading (g) \\
\hline 0 & 0 & 0 & 0.00 \\
\hline 10.40 & 0.10 & -0.10 & -10.19 \\
\hline 25.35 & 0.25 & -0.25 & -25.48 \\
\hline 35.35 & 0.35 & -0.35 & -35.68 \\
\hline 70.65 & 0.69 & -0.69 & -70.34 \\
\hline 93.35 & 0.92 & -0.92 & -93.78 \\
\hline 208.40 & 2.04 & -2.03 & -206.93 \\
\hline 323.80 & 3.18 & -3.18 & -324.16 \\
\hline 552.10 & 5.42 & -5.43 & -553.52 \\
\hline 779.45 & 7.65 & -7.68 & -782.87 \\
\hline 1006.80 & 9.88 & -9.92 & -1011.21 \\
\hline
\end{tabular}


SM-25LBS-1 Serial Number: 672365

\begin{tabular}{|c|c|c|c|c|}
\hline HX711 Amp \# & 2 & & & \\
\hline $\begin{array}{l}\text { Amp Calibration } \\
\text { Factor }\end{array}$ & -66570 & & & \\
\hline Load Cell Zero & $\sim 1.11 \mathrm{~N}$ & $\begin{array}{l}\text { Load Cell } \\
\text { Accuracy }\end{array}$ & $\sim 0.0075 \mathrm{Lbs}$ & $\sim 0.0033 \mathrm{~N}$ \\
\hline \multicolumn{5}{|l|}{ Tension } \\
\hline Weight (g) & Load $(\mathrm{N})$ & Reading (N) & Reading (g) & \\
\hline 0.00 & 0.000 & 0.000 & 0.00 & \\
\hline 115.35 & 1.132 & -1.133 & -115.49 & \\
\hline 227.60 & 2.233 & -2.233 & -227.62 & \\
\hline 343.00 & 3.365 & -3.366 & -343.12 & \\
\hline 455.90 & 4.472 & -4.473 & -455.96 & \\
\hline 570.35 & 5.595 & -5.596 & -570.44 & \\
\hline 683.25 & 6.703 & -6.706 & -683.59 & \\
\hline \multicolumn{5}{|l|}{ Compression } \\
\hline Weight (g) & Load $(\mathrm{N})$ & Reading (N) & Reading (g) & \\
\hline 0 & 0.000 & 0.000 & 0.00 & \\
\hline 115.35 & 1.132 & 1.133 & 115.49 & \\
\hline 227.60 & 2.233 & 2.233 & 227.62 & \\
\hline 342.95 & 3.364 & 3.364 & 342.92 & \\
\hline 455.85 & 4.472 & 4.471 & 455.76 & \\
\hline 571.20 & 5.603 & 5.603 & 571.15 & \\
\hline 686.30 & 6.733 & 6.732 & 686.24 & \\
\hline 798.60 & 7.834 & 7.833 & 798.47 & \\
\hline 913.70 & 8.963 & 8.962 & 913.56 & \\
\hline 1141.00 & 11.193 & 11.193 & 1140.98 & \\
\hline
\end{tabular}


SM-25LBS-2 Serial Number: 1010237

\begin{tabular}{|c|c|c|c|c|}
\hline Scale Accuracy & $0.05 \mathrm{~g}$ & & & \\
\hline HX711 Amp \# & 3 & & & \\
\hline $\begin{array}{l}\text { Amp Calibration } \\
\text { Factor }\end{array}$ & -65050 & & & \\
\hline Load Cell Zero & $\sim 1.11 \mathrm{~N}$ & $\begin{array}{l}\text { Load Cell } \\
\text { Accuracy }\end{array}$ & $\sim 0.0075$ Lbs & $\sim 0.0033 \mathrm{~N}$ \\
\hline \multicolumn{5}{|l|}{ Tension } \\
\hline Weight (g) & Load (N) & Reading (N) & Reading (g) & \\
\hline 0.00 & 0.000 & 0.000 & 0.00 & \\
\hline 115.35 & 1.132 & -1.132 & -115.39 & \\
\hline 228.35 & 2.240 & -2.241 & -228.44 & \\
\hline 343.70 & 3.372 & -3.374 & -343.93 & \\
\hline 455.90 & 4.472 & -4.476 & -456.27 & \\
\hline 571.25 & 5.604 & -5.610 & -571.87 & \\
\hline 683.20 & 6.702 & -6.709 & -683.89 & \\
\hline \multicolumn{5}{|l|}{ Compression } \\
\hline Weight (g) & Load $(\mathrm{N})$ & Reading (N) & Reading (g) & \\
\hline 0 & 0.000 & 0.000 & 0.00 & \\
\hline 115.35 & 1.132 & 1.133 & 115.49 & \\
\hline 227.35 & 2.230 & 2.231 & 227.42 & \\
\hline 342.65 & 3.361 & 3.363 & 342.81 & \\
\hline 455.65 & 4.470 & 4.472 & 455.86 & \\
\hline 570.95 & 5.601 & 5.605 & 571.36 & \\
\hline 798.60 & 7.834 & 7.845 & 799.69 & \\
\hline 913.65 & 8.963 & 8.977 & 915.09 & \\
\hline 1141.05 & 11.194 & 11.210 & 1142.71 & \\
\hline
\end{tabular}




\section{Speed Calibration}

The Actuonix LAC Board has a dial with 11 settings. Settings 1 through 4 produced step displacement. Setting 5 is the slowest speed setting to produce a smooth continuous displacement.

Setting 5

\begin{tabular}{|l|l|}
\hline Average Trial 1 & 0.0117 \\
\hline Average Trial 2 & 0.0115 \\
\hline $\begin{array}{l}\text { Average of } \\
\text { Trials }\end{array}$ & 0.0116 \\
\hline
\end{tabular}

\section{Trial 1}
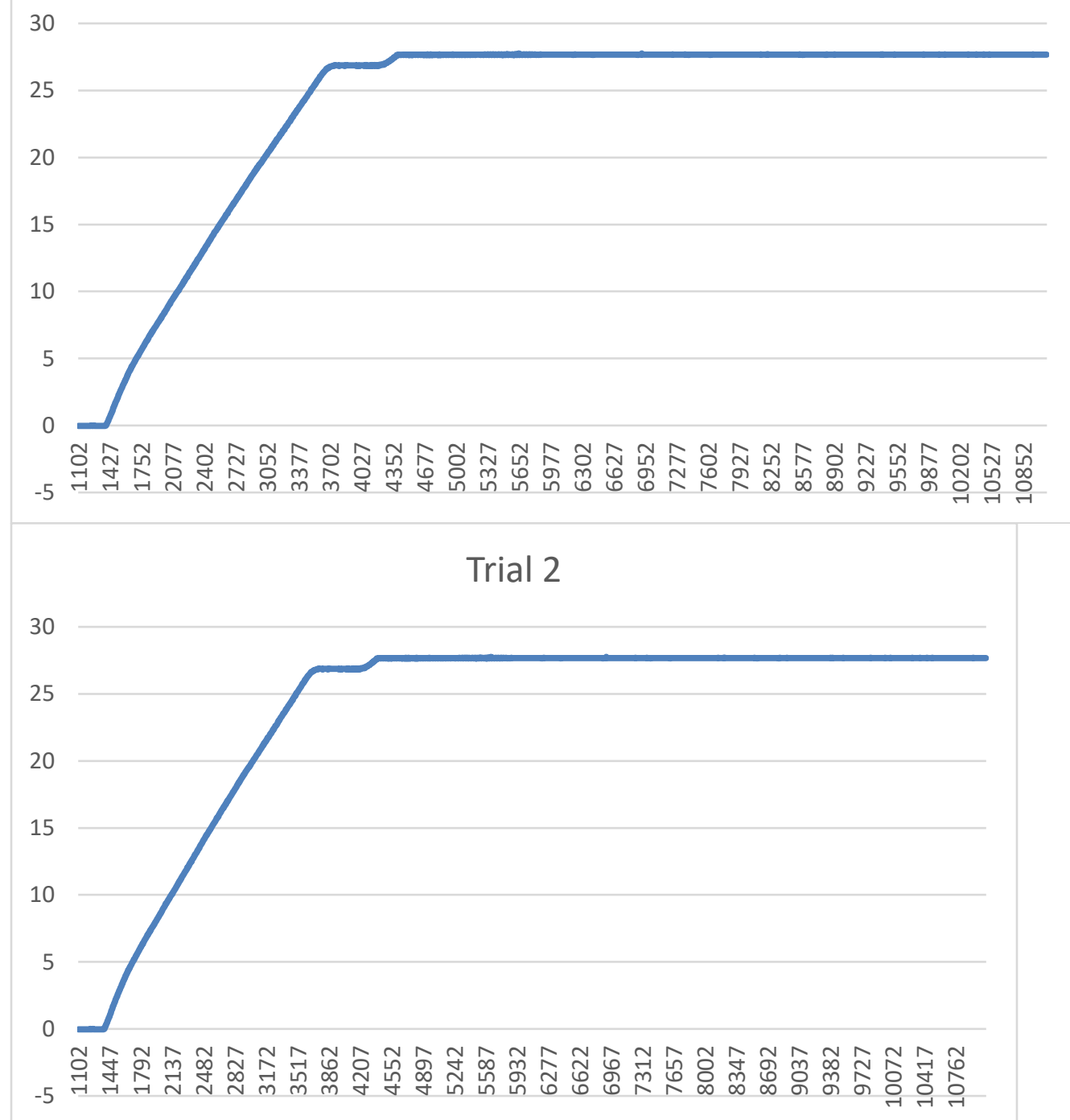


Setting 6
\begin{tabular}{|l|l|}
\hline Average Trial 1 & 0.0159 \\
\hline Average Trial 2 & 0.0165 \\
\hline $\begin{array}{l}\text { Average of } \\
\text { Trials }\end{array}$ & 0.0162 \\
\hline
\end{tabular}
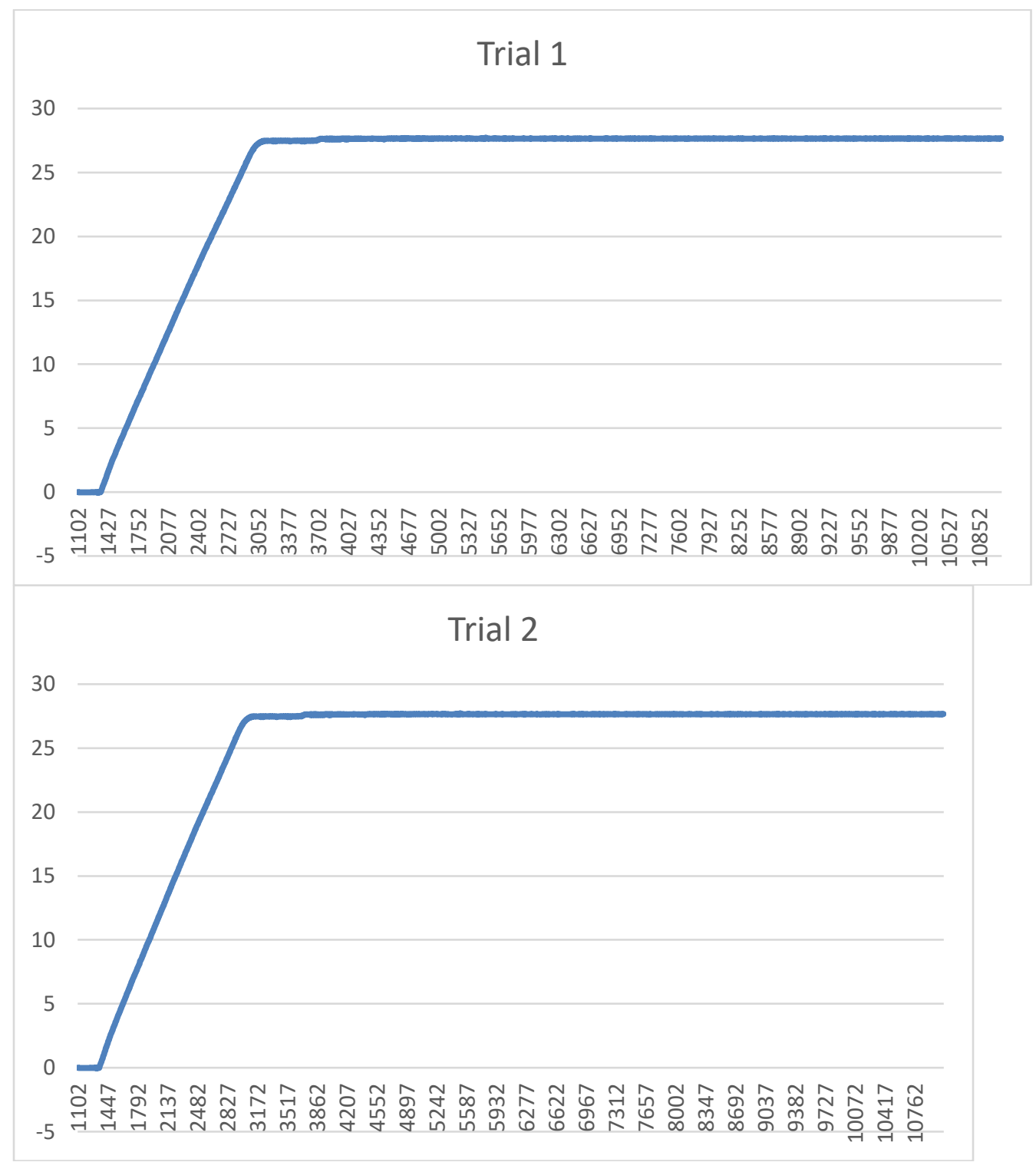
Setting 7

\begin{tabular}{|l|l|}
\hline Average Trial 1 & 0.0193 \\
\hline Average Trial 2 & 0.0193 \\
\hline $\begin{array}{l}\text { Average of } \\
\text { Trials }\end{array}$ & 0.0193 \\
\hline
\end{tabular}

\section{Trial 1}
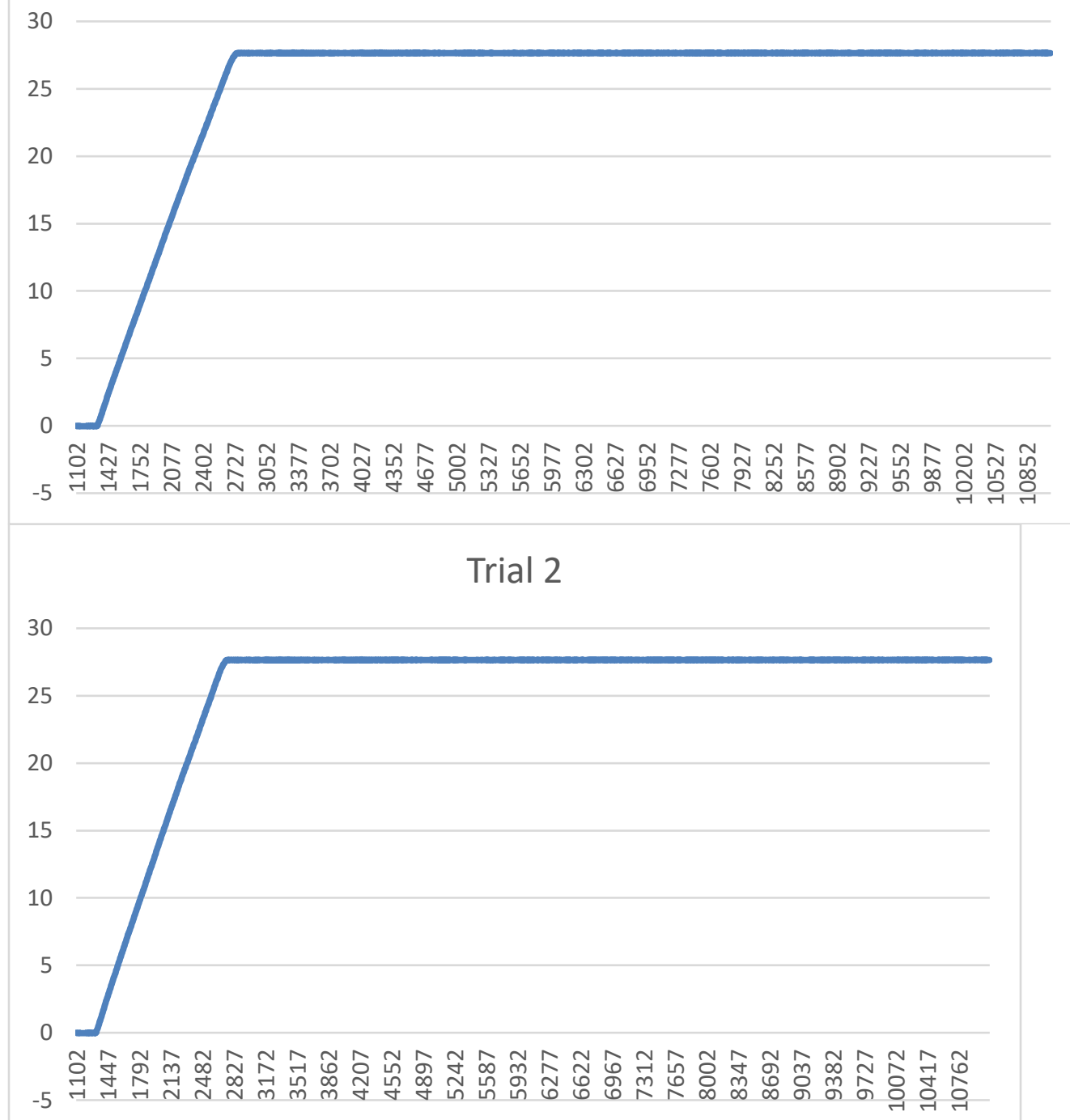


Setting 8
\begin{tabular}{|l|l|}
\hline Average Trial 1 & 0.0197 \\
\hline Average Trial 2 & 0.0202 \\
\hline $\begin{array}{l}\text { Average of } \\
\text { Trials }\end{array}$ & 0.0199 \\
\hline
\end{tabular}
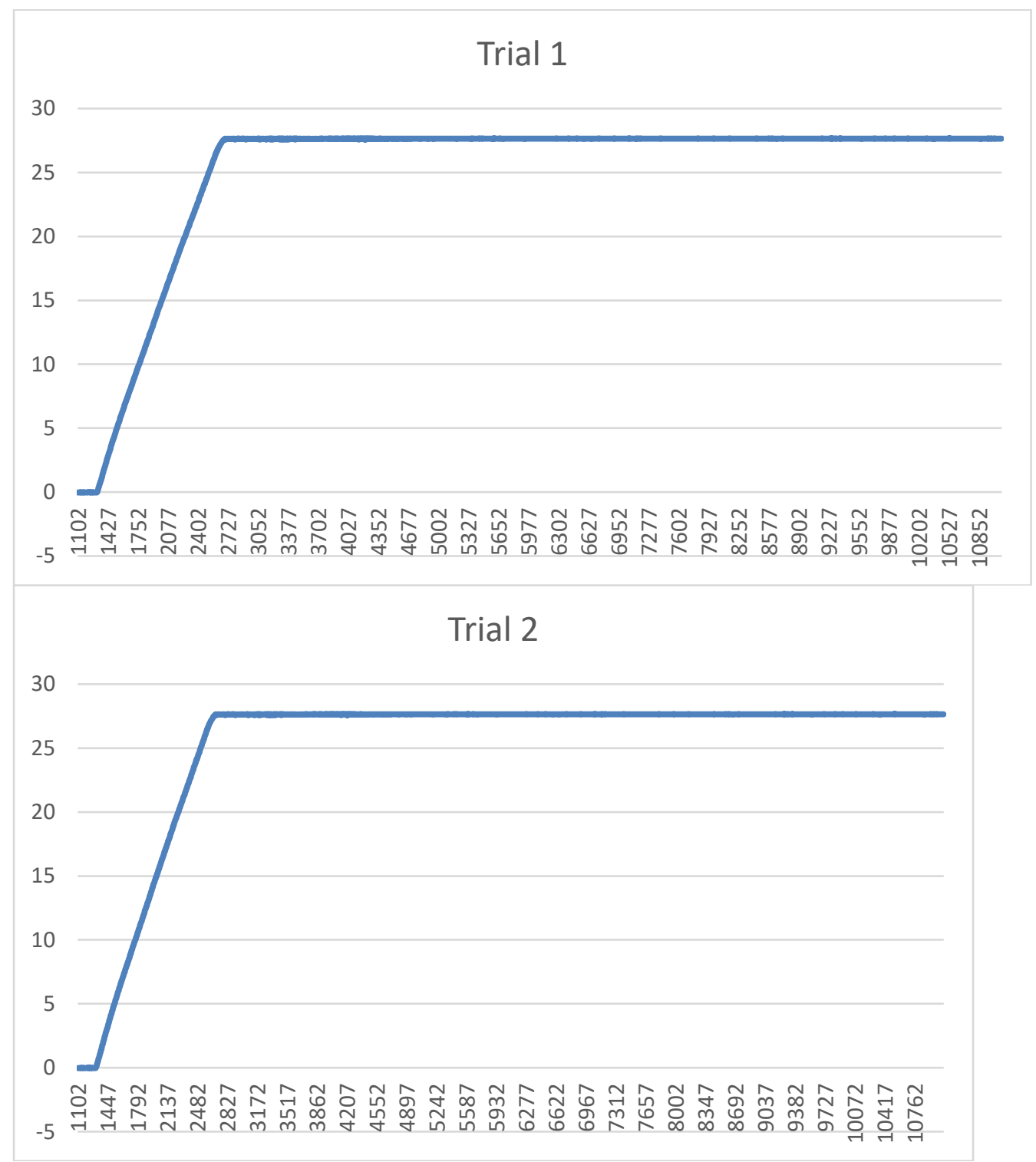


Setting 9
\begin{tabular}{|l|l|}
\hline Average Trial 1 & 0.0235 \\
\hline Average Trial 2 & 0.0237 \\
\hline $\begin{array}{l}\text { Average of } \\
\text { Trials }\end{array}$ & 0.0236 \\
\hline
\end{tabular}
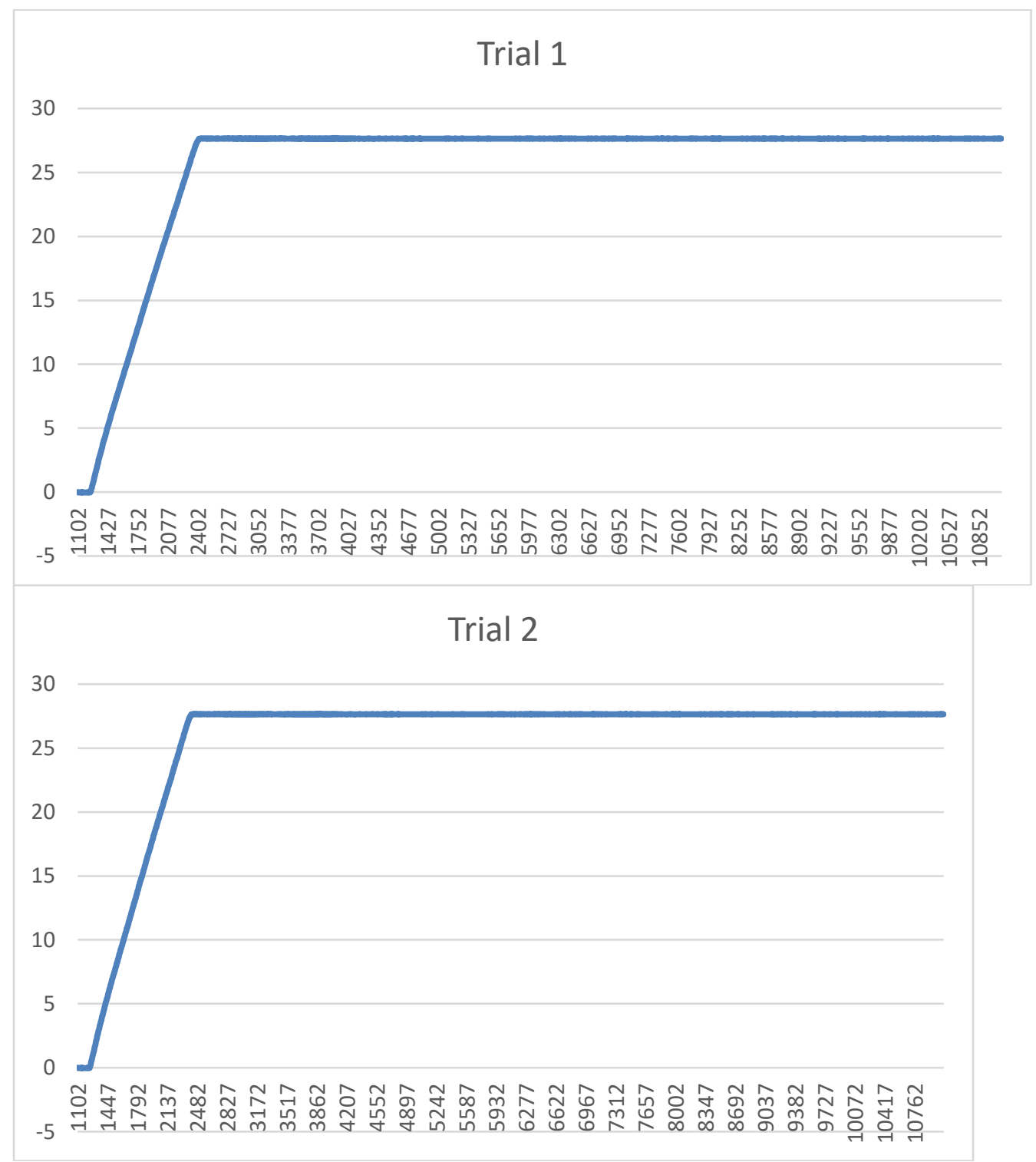


Setting 10
\begin{tabular}{|l|l|}
\hline Average Trial 1 & 0.0234 \\
\hline Average Trial 2 & 0.0253 \\
\hline $\begin{array}{l}\text { Average of } \\
\text { Trials }\end{array}$ & 0.0243 \\
\hline
\end{tabular}
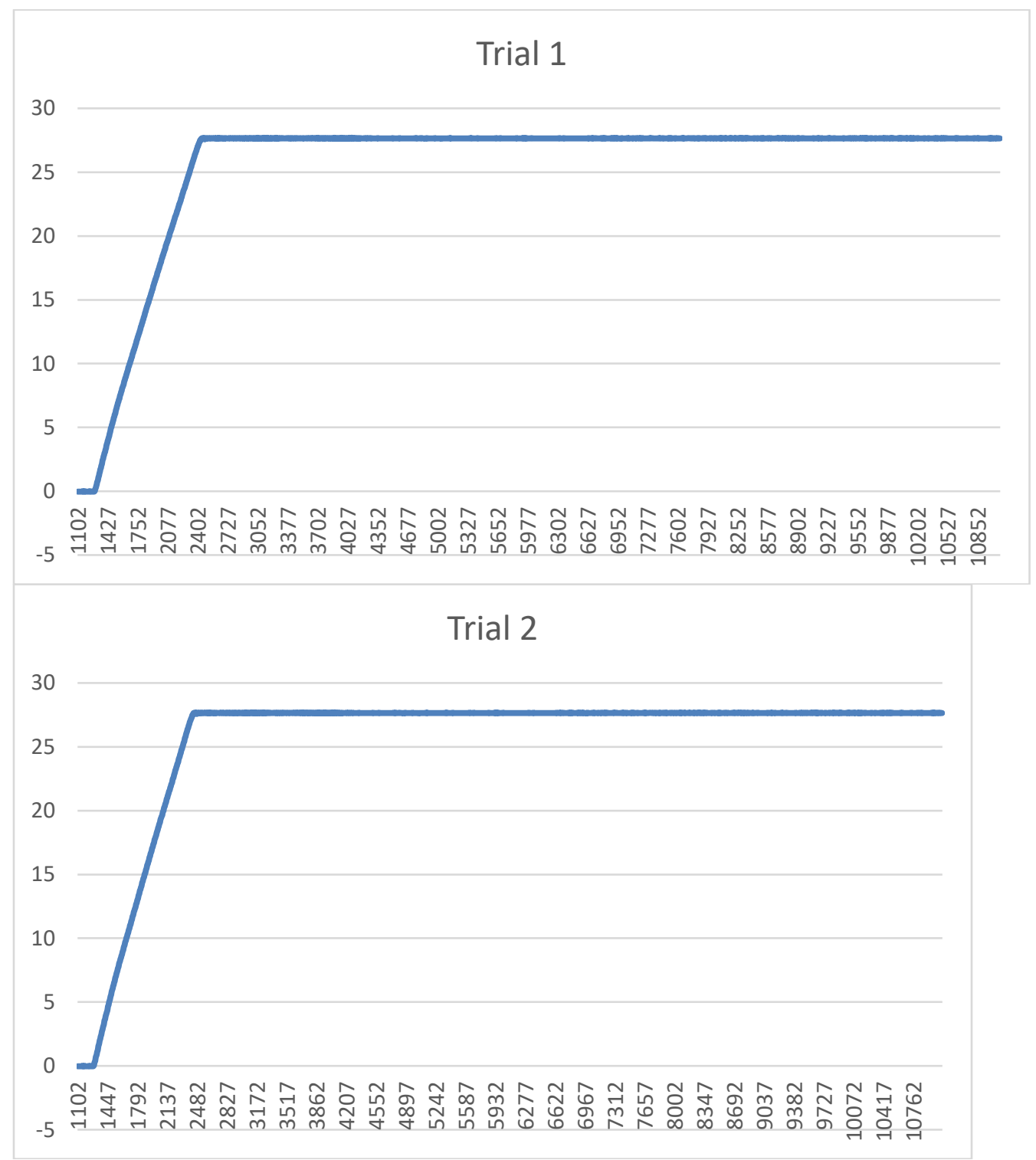


\section{Setting 11}

\begin{tabular}{|l|l|}
\hline Average Trial 1 & 0.0262 \\
\hline Average Trial 2 & 0.0262 \\
\hline $\begin{array}{l}\text { Average of } \\
\text { Trials }\end{array}$ & 0.0262 \\
\hline
\end{tabular}

\section{Trial 1}
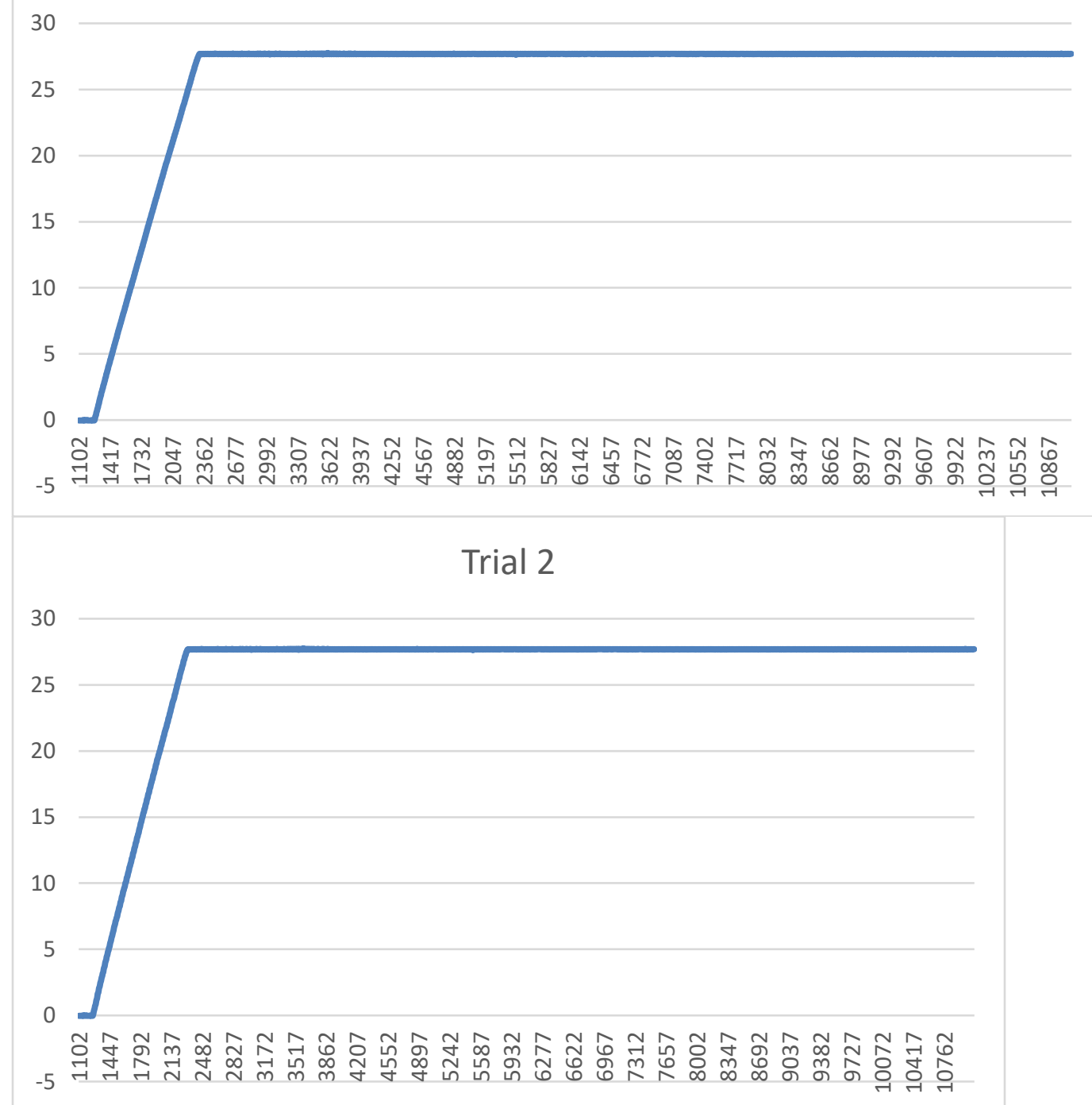
Stress Relaxation and Indentation

No indentation

\begin{tabular}{|c|c|c|c|c|c|c|c|}
\hline \multicolumn{2}{|c|}{ Reading 1 } & \multicolumn{2}{c|}{ Reading 2 } & \multicolumn{2}{c|}{ Reading 3 } & \multicolumn{2}{c|}{ Reading 4 } \\
\hline SM-25-1 & SM-25-2 & SM-25-1 & SM-25-2 & SM-25-1 & SM-25-2 & SM-25-1 & SM-25-2 \\
\hline-10.65 & -16.39 & -9.8 & -14.86 & -9.46 & -13.85 & -9.19 & -13.02 \\
\hline Time & 106864 & Time & 384174 & Time & 1136725 & Time & 2481478 \\
\hline \multicolumn{2}{|c|}{ Reading 5 } & \multicolumn{2}{|c|}{ Reading 6 } & \multicolumn{2}{c|}{ Reading 7 } & \multicolumn{2}{c|}{ Reading 8 } \\
\hline SM-25-1 & SM-25-2 & SM-25-1 & SM-25-2 & SM-25-1 & SM-25-2 & SM-25-1 & SM-25-2 \\
\hline-9.08 & -12.69 & -9.01 & -12.41 & -8.93 & -12.24 & -8.9 & -12.01 \\
\hline Time & 3257600 & Time & 4118350 & Time & 4705060 & Time & 5731208 \\
\hline
\end{tabular}

With Indentation 3 locations per testing time

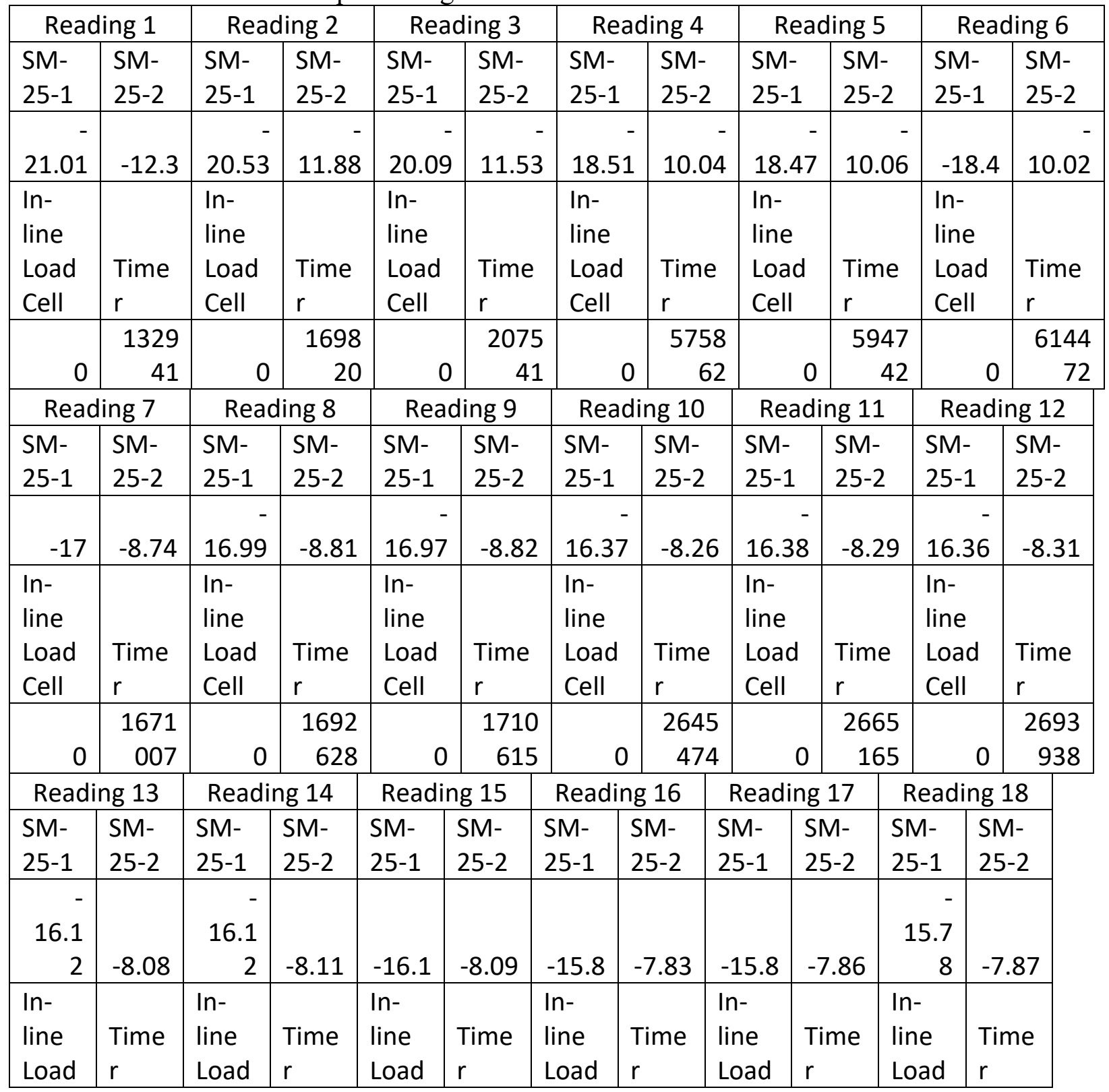




\begin{tabular}{|r|r|r|r|r|r|r|r|r|r|r|r|}
\hline Cell & & Cell & & Cell & & Cell & & Cell & & Cell & \\
\hline & 3191 & & 3209 & & 3226 & & 4113 & & 4133 & & 4151 \\
0 & 122 & 0 & 064 & 0 & 092 & 0 & 194 & 0 & 830 & 0 & 775 \\
\hline
\end{tabular}

Stress Relaxation no Indenter

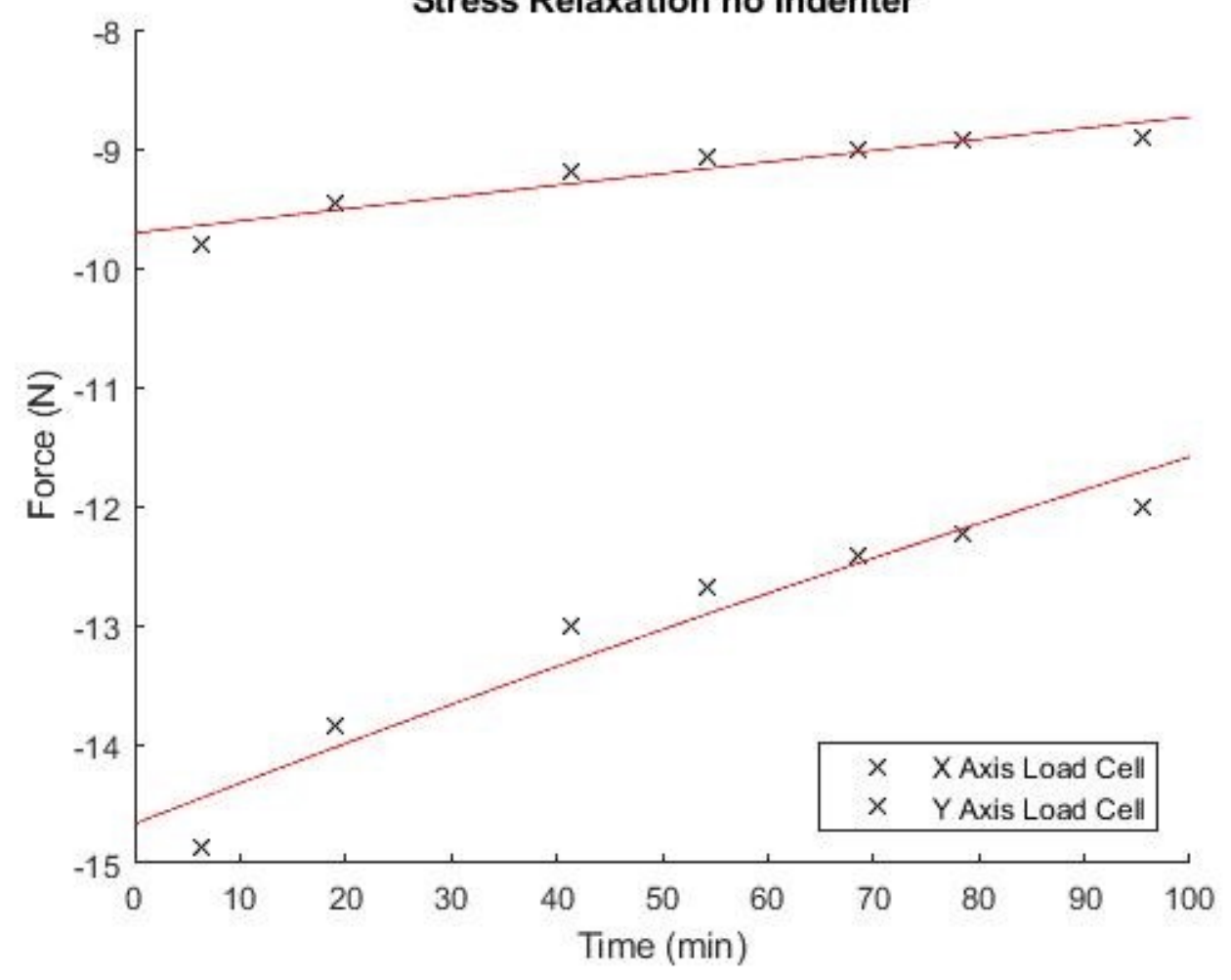



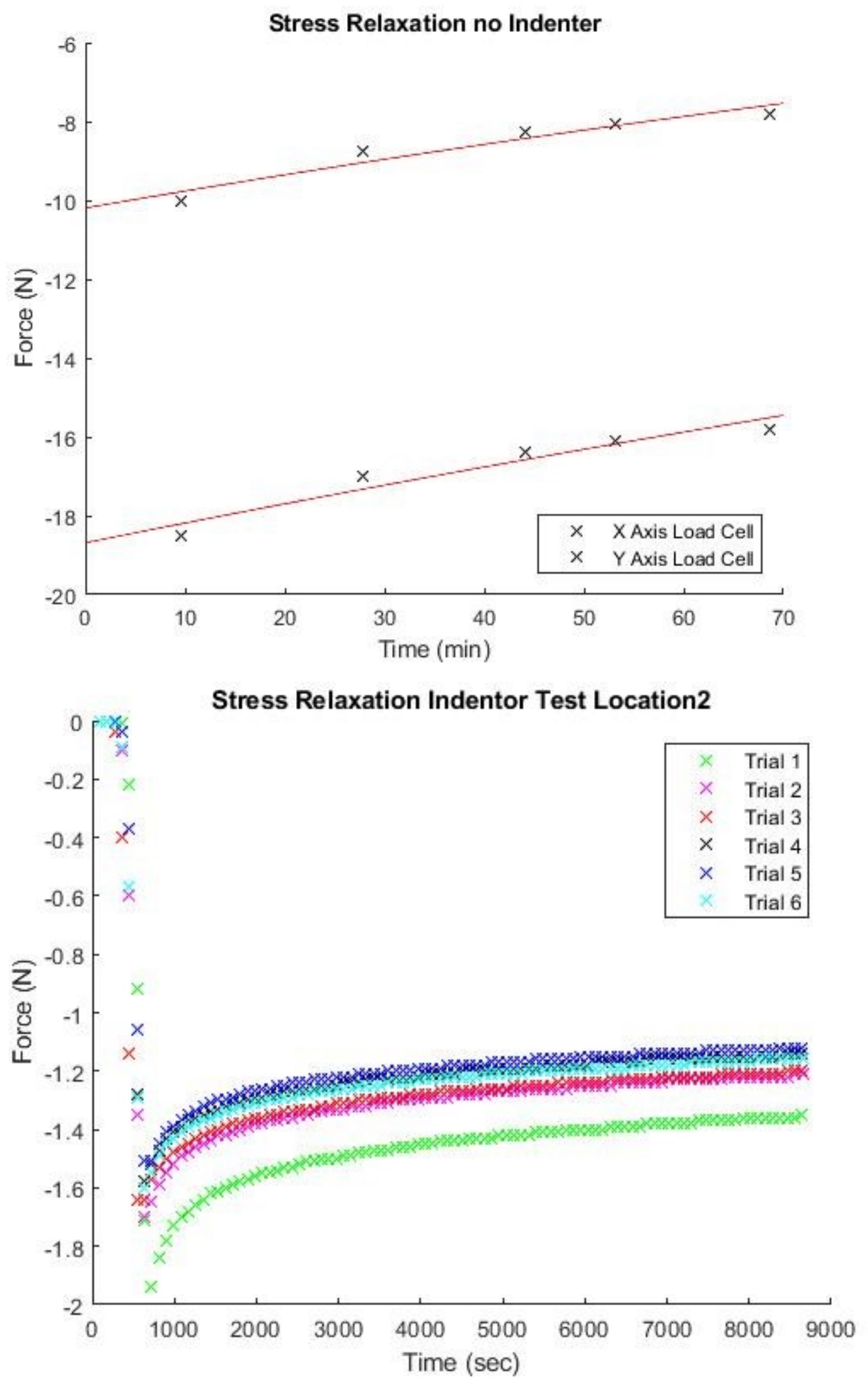

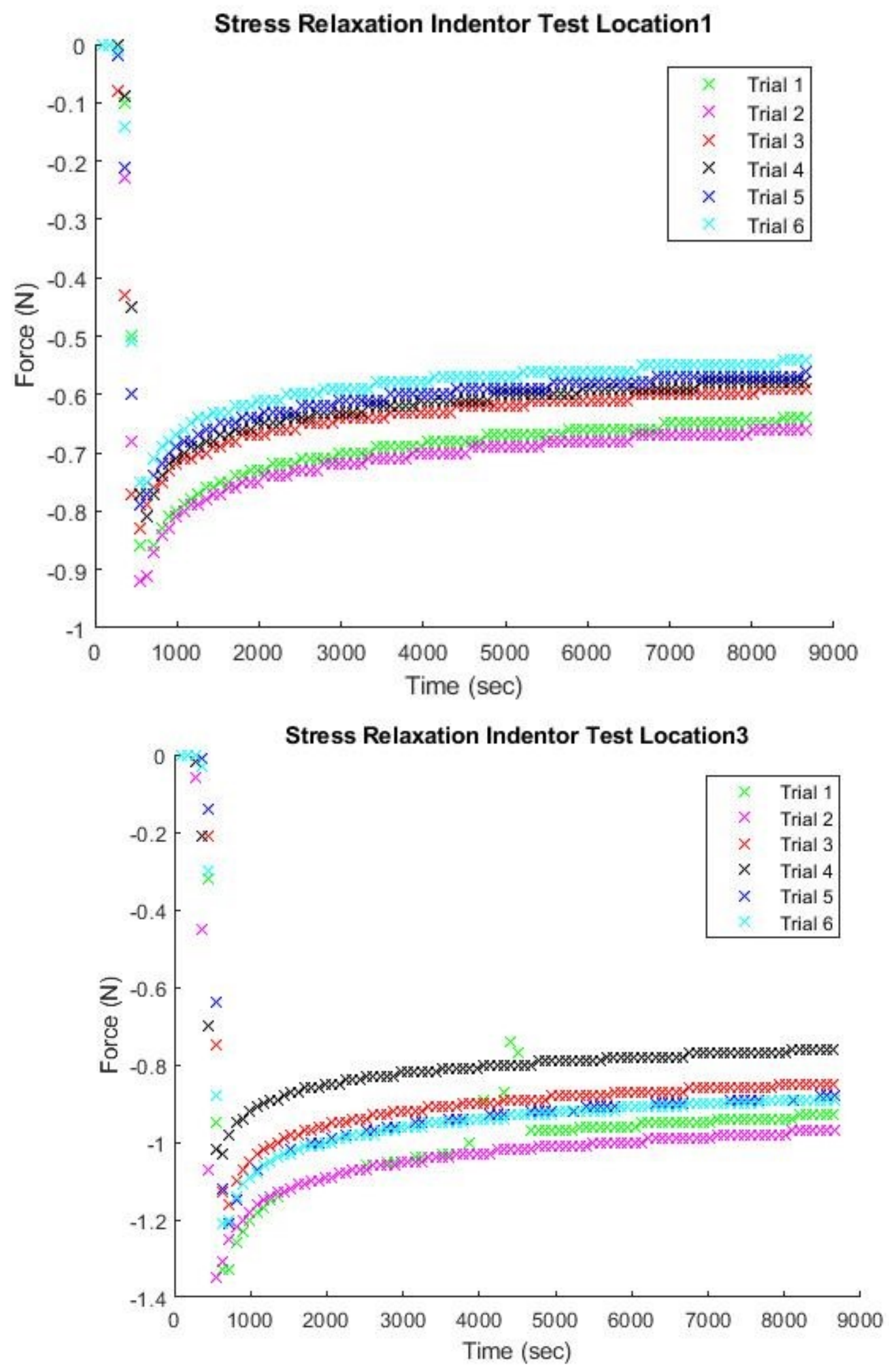
Fracture
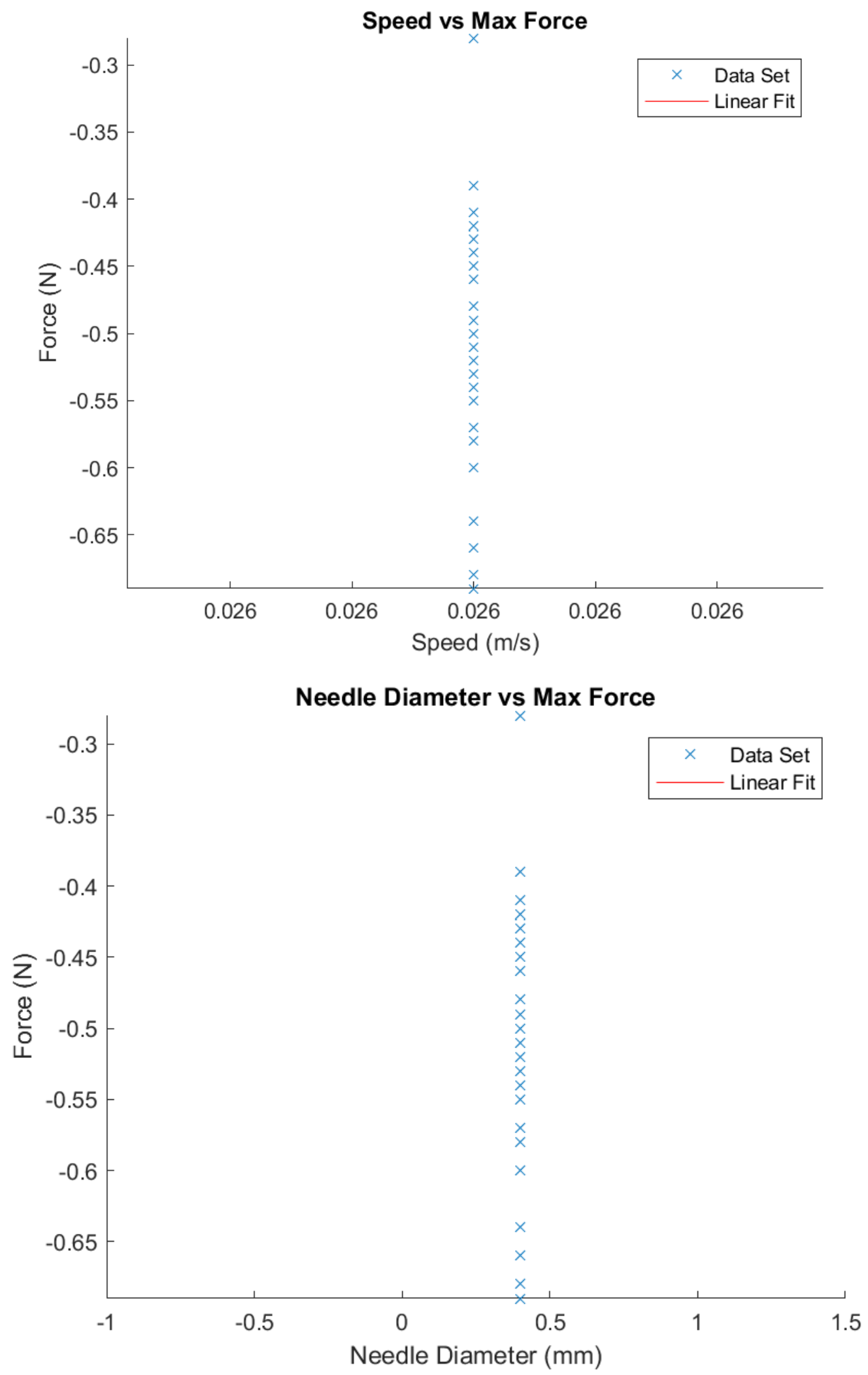


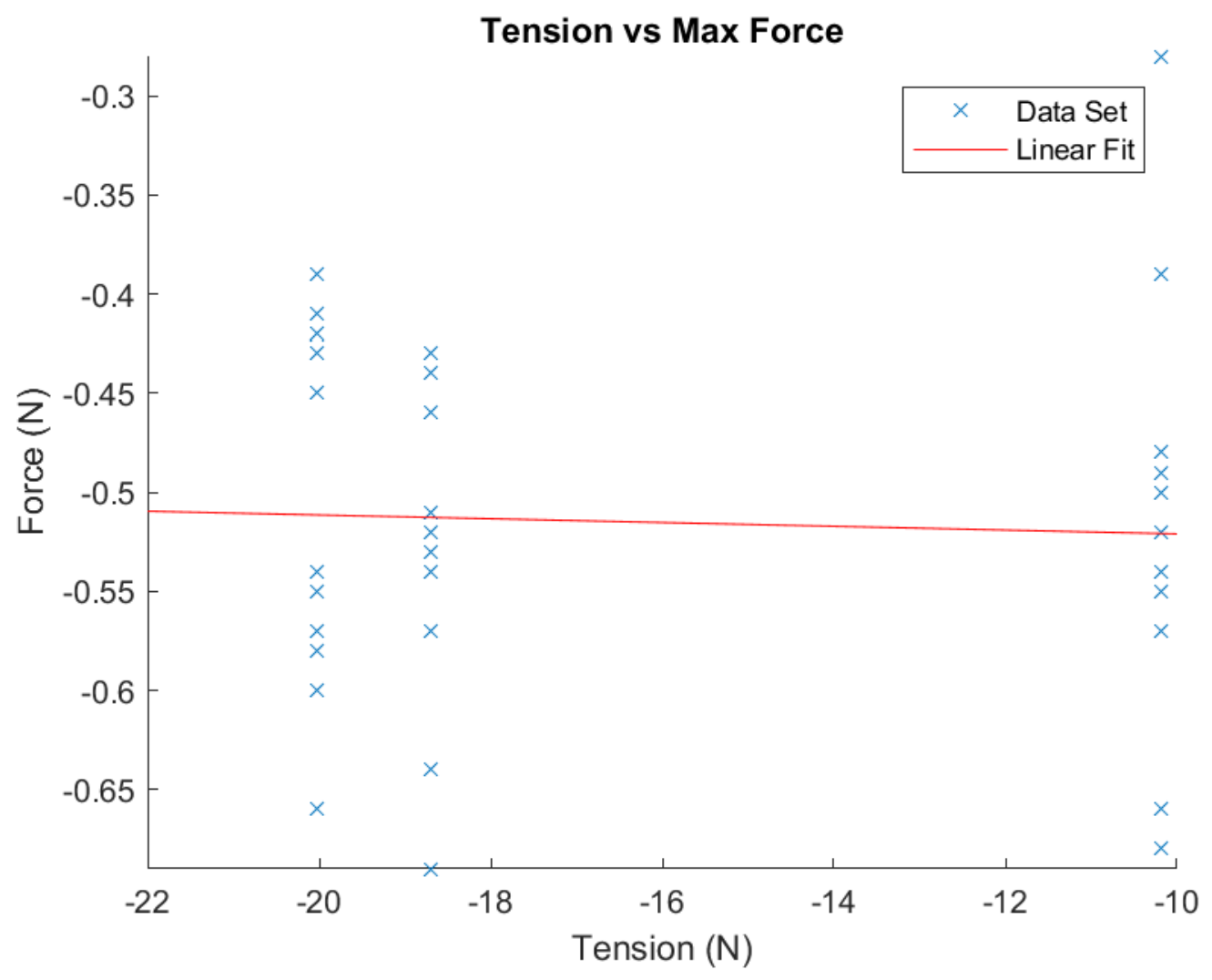




\section{Appendix D - Matlab and Arduino Codes}

This appendix gives the Matlab and Arduino codes used in the data processing. The Matlab codes require all the raw data excel files to be in one folder before use. Excel files must be specifically formatted for the code to work properly.

\section{Matlab Codes}

SneddonConeSurface.m

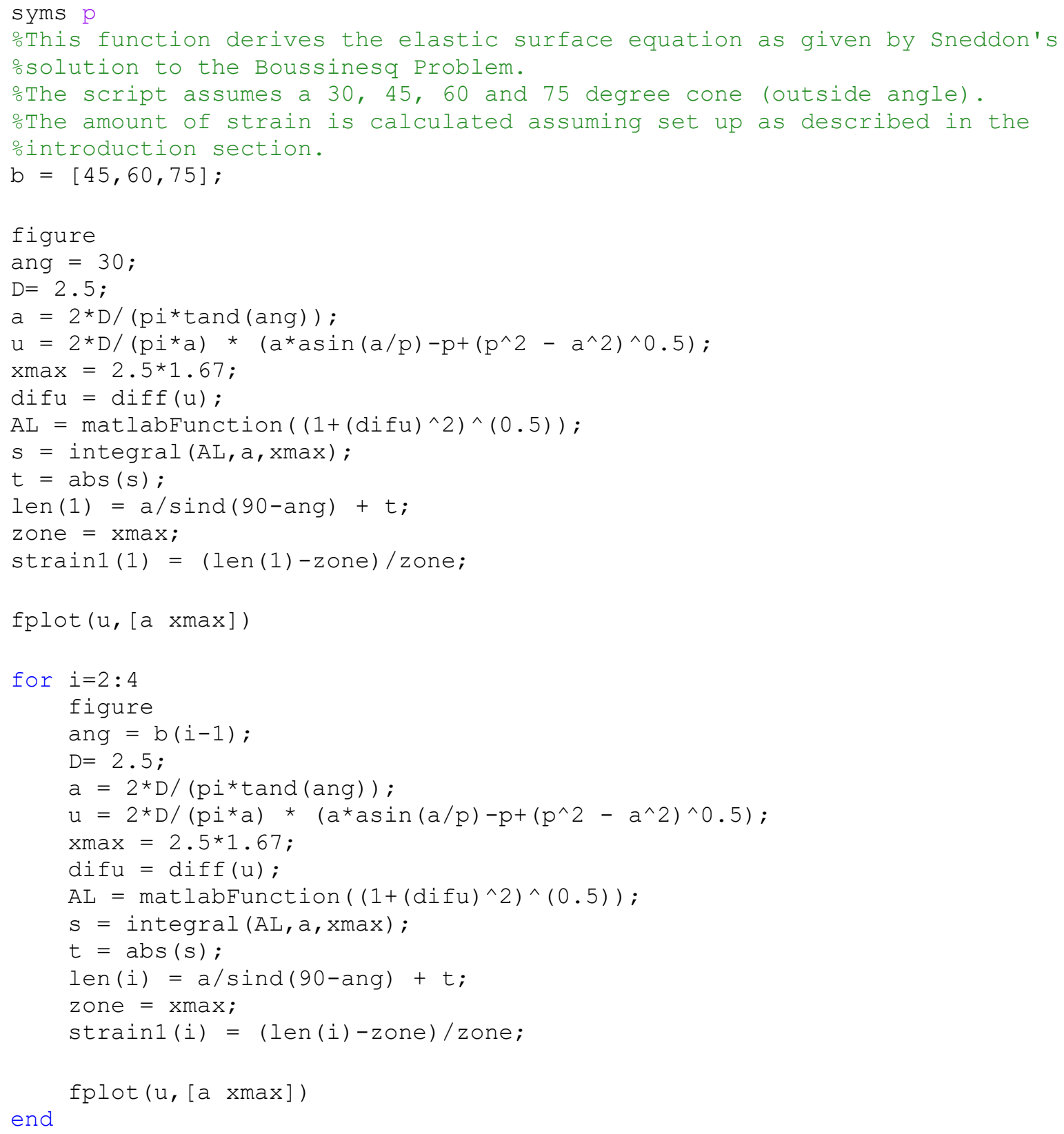




\section{SneddonClySurface.m}

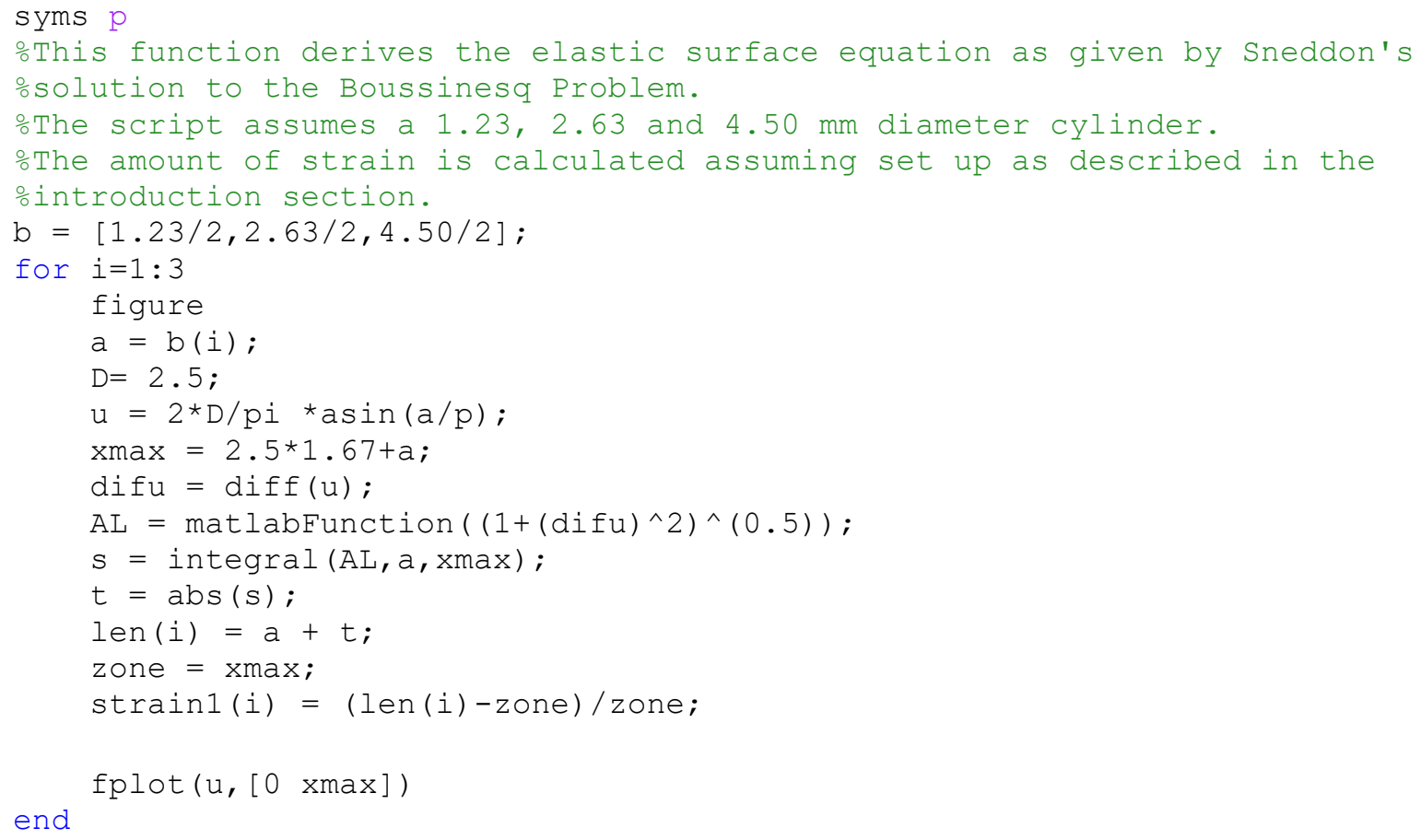




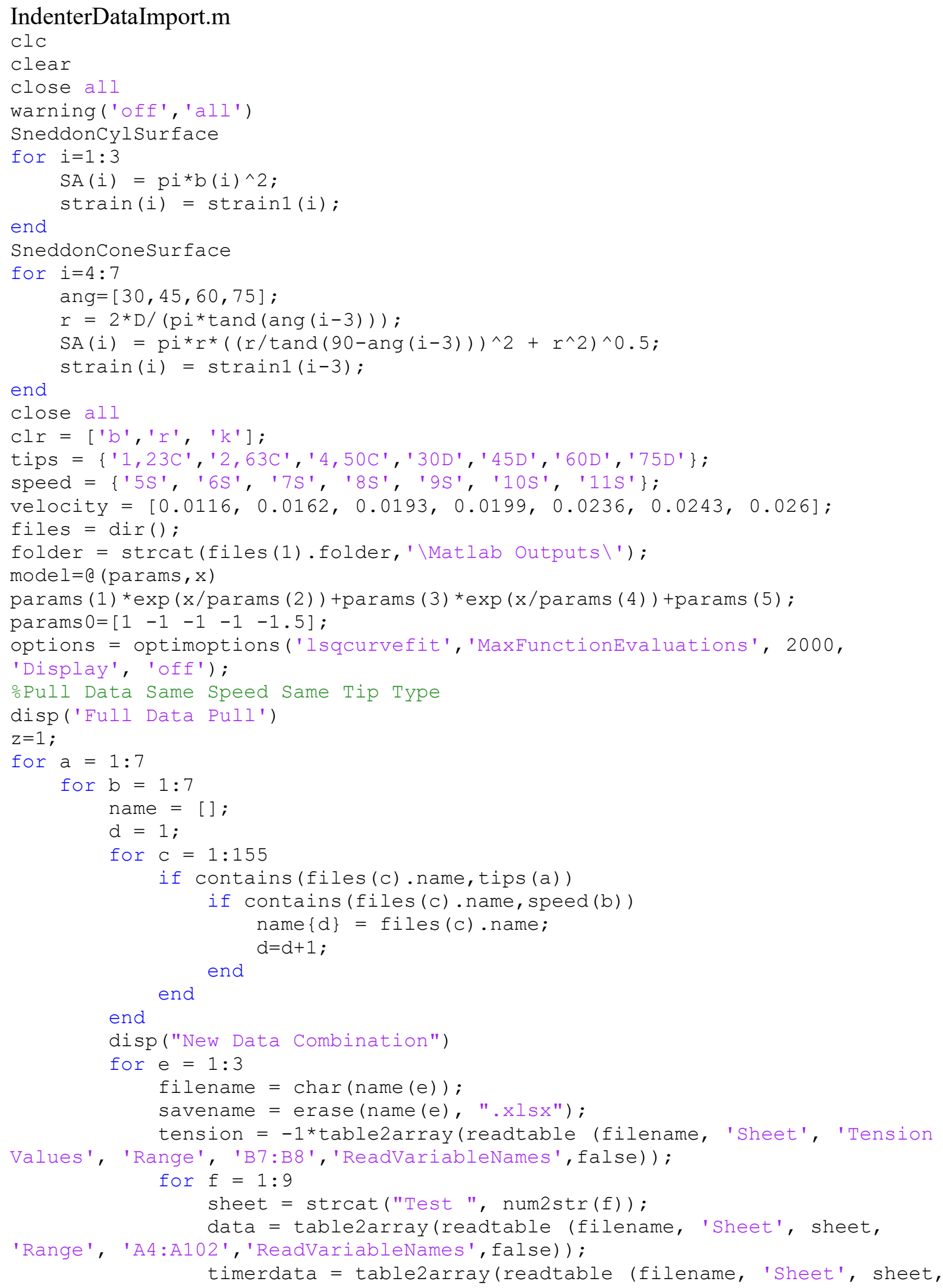




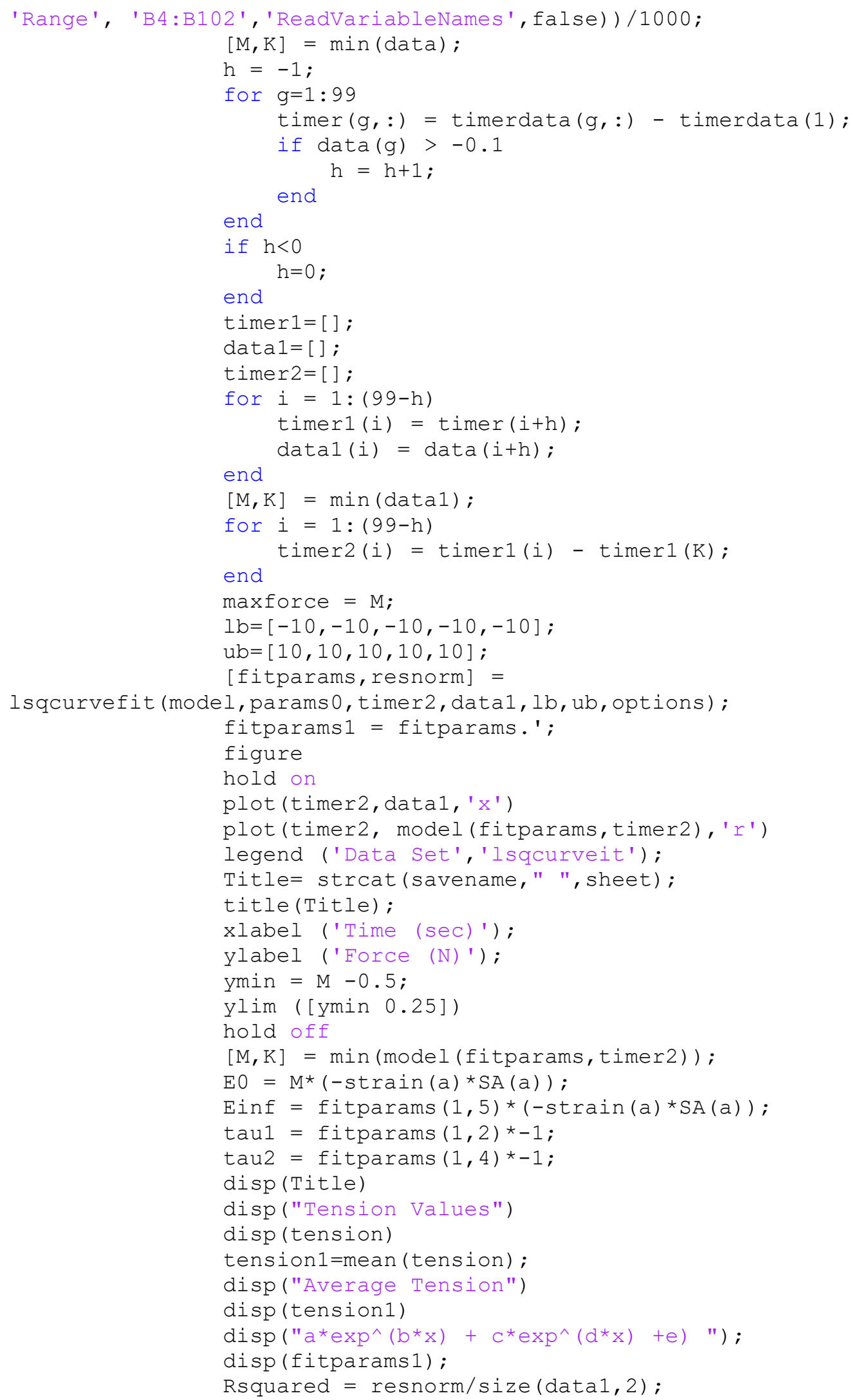




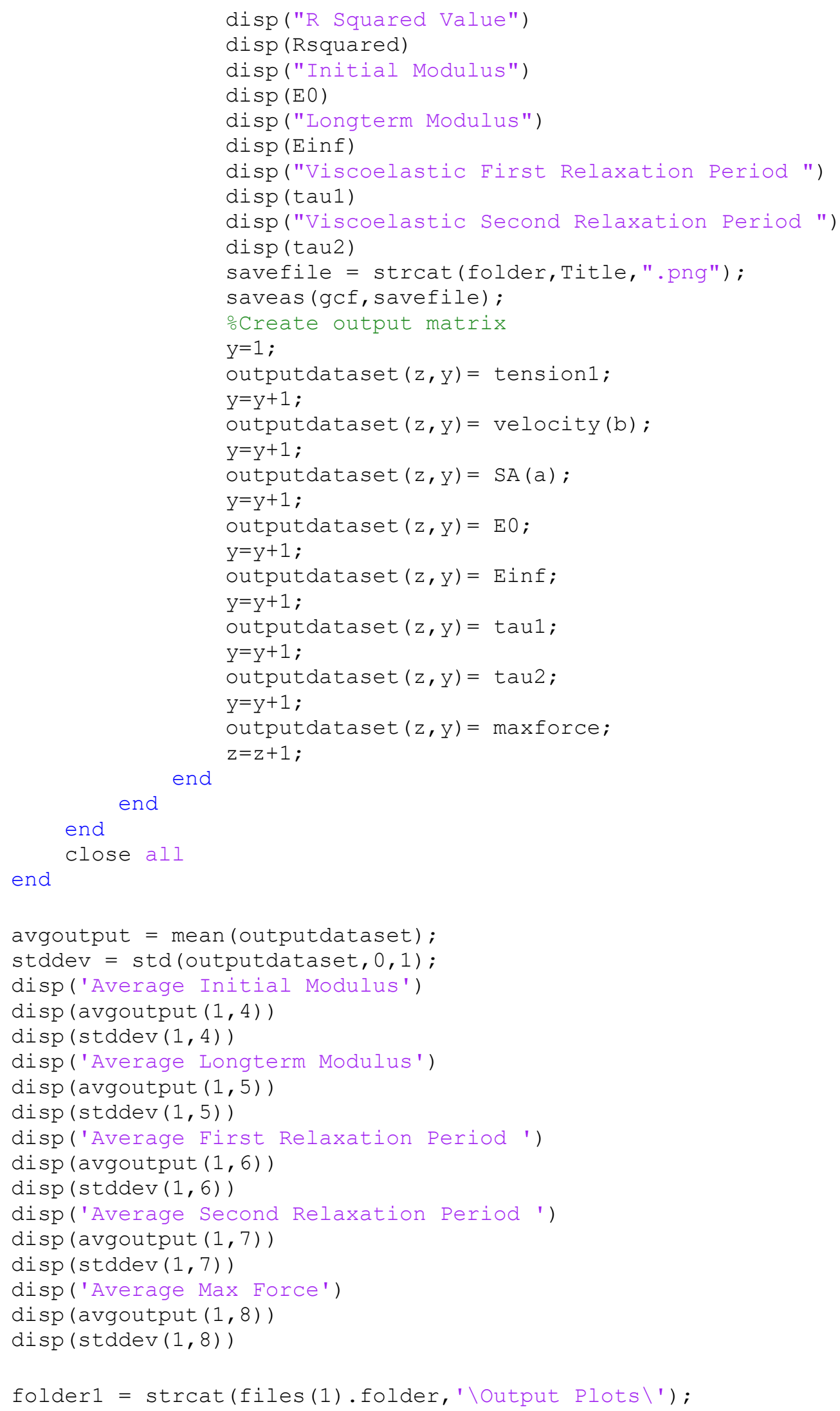




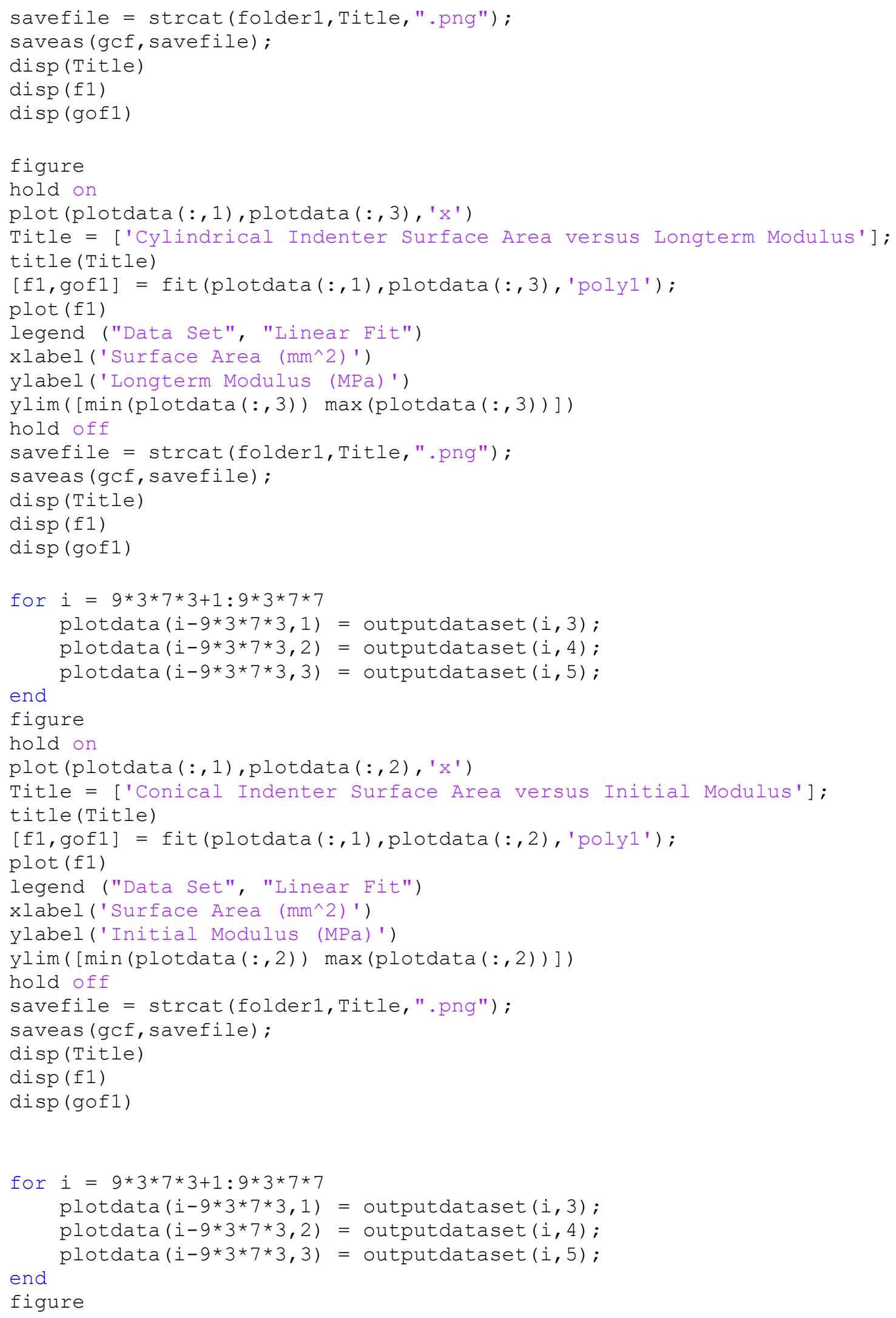




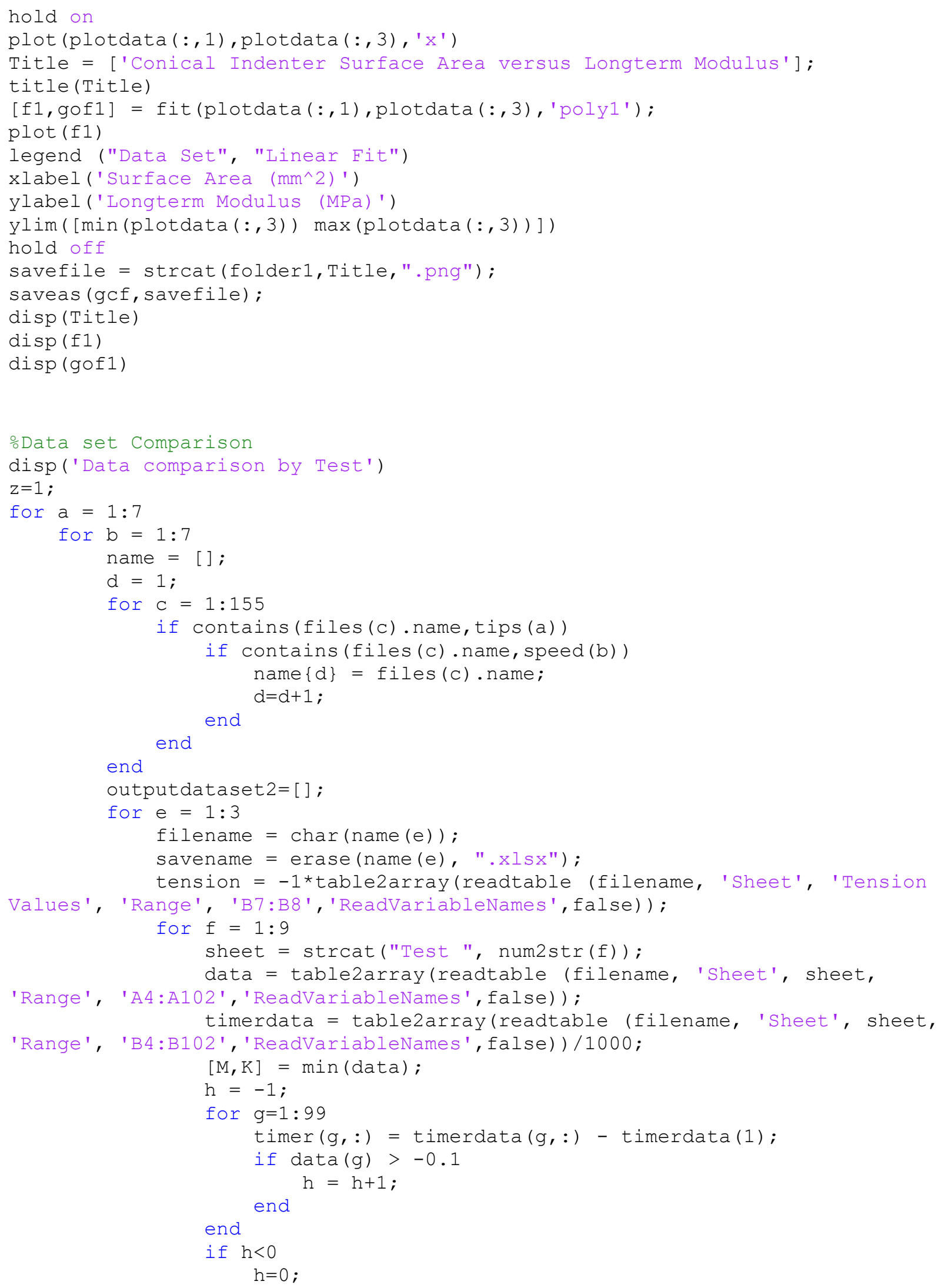




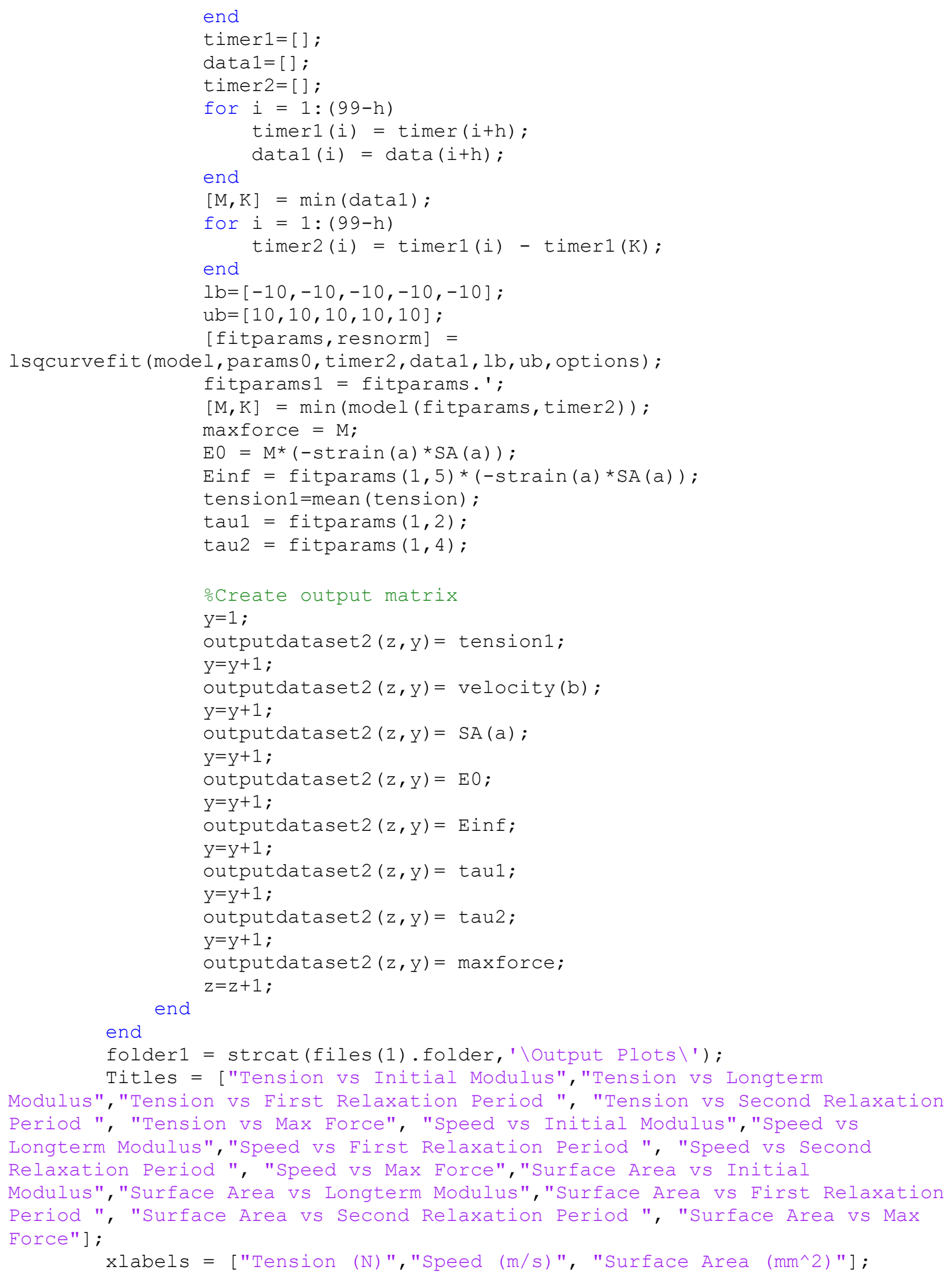




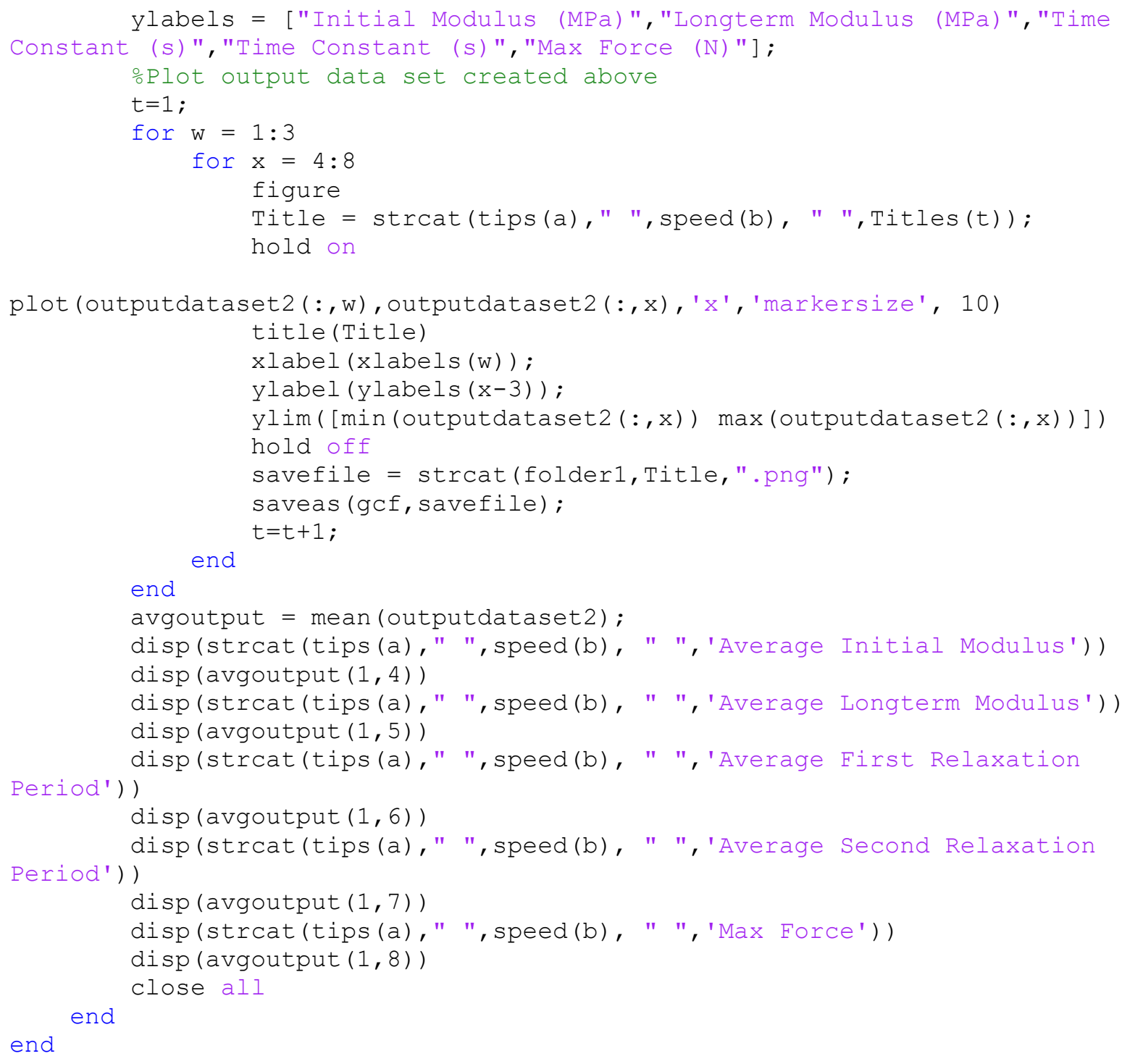




\section{FractureDataImport.m}

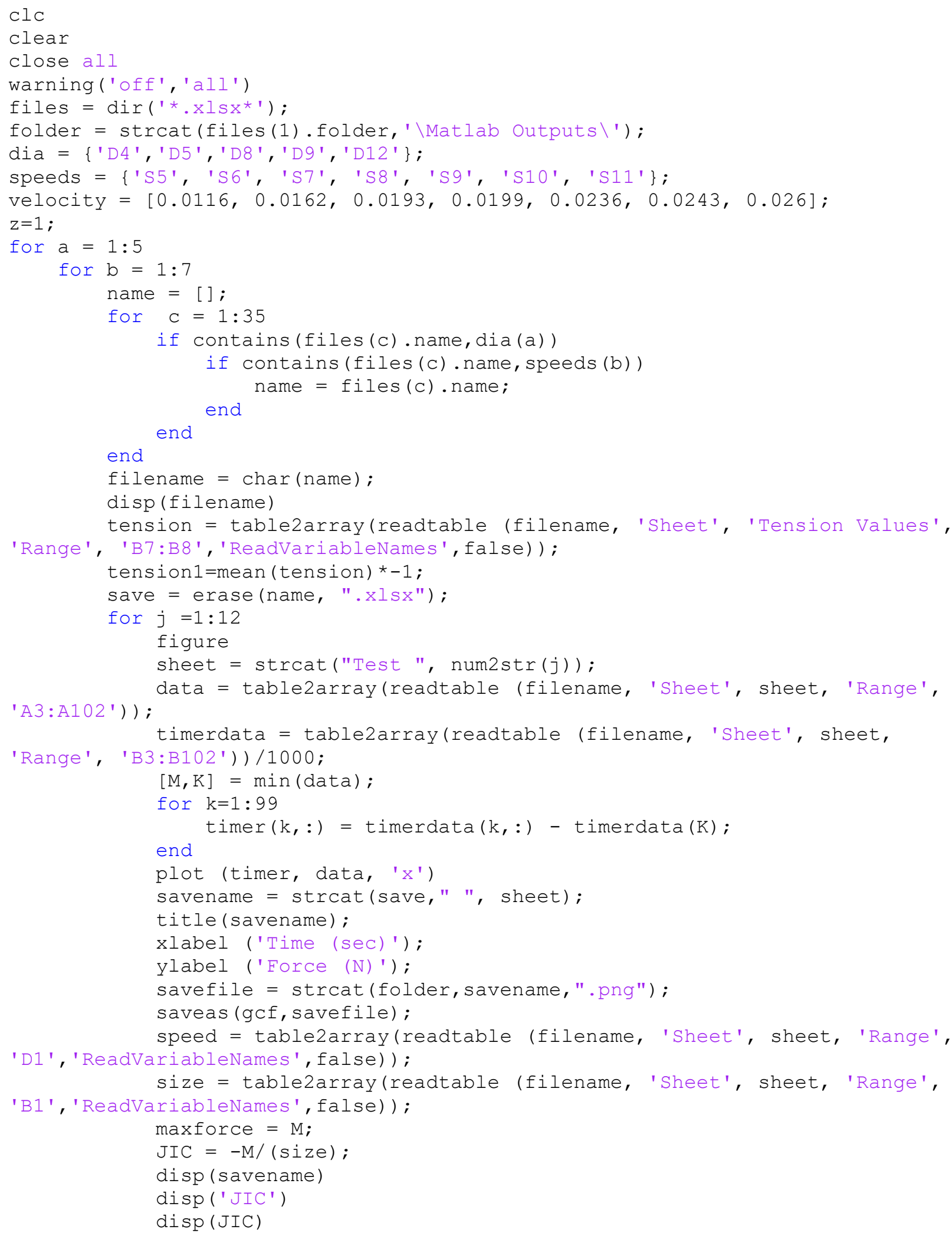




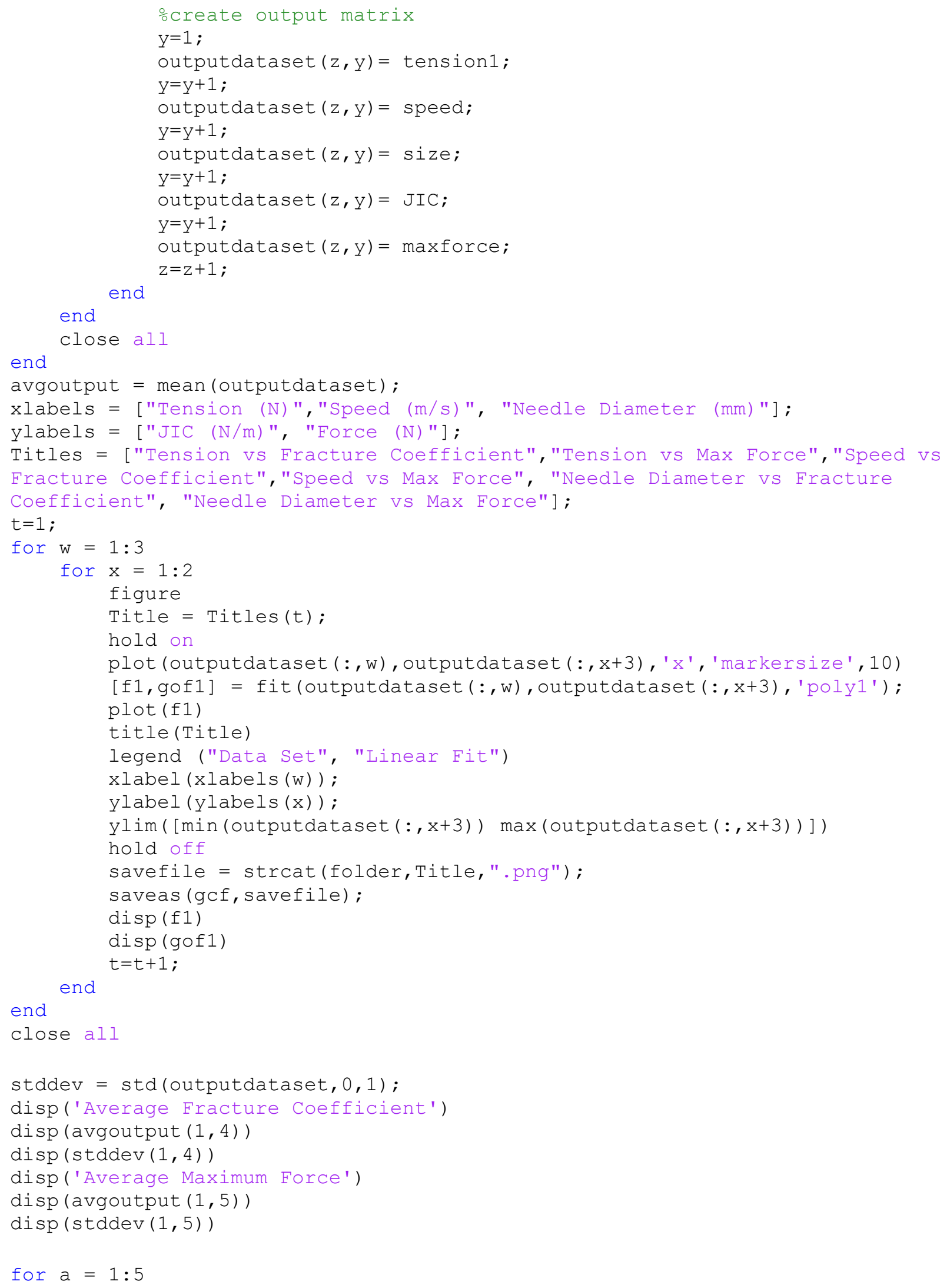




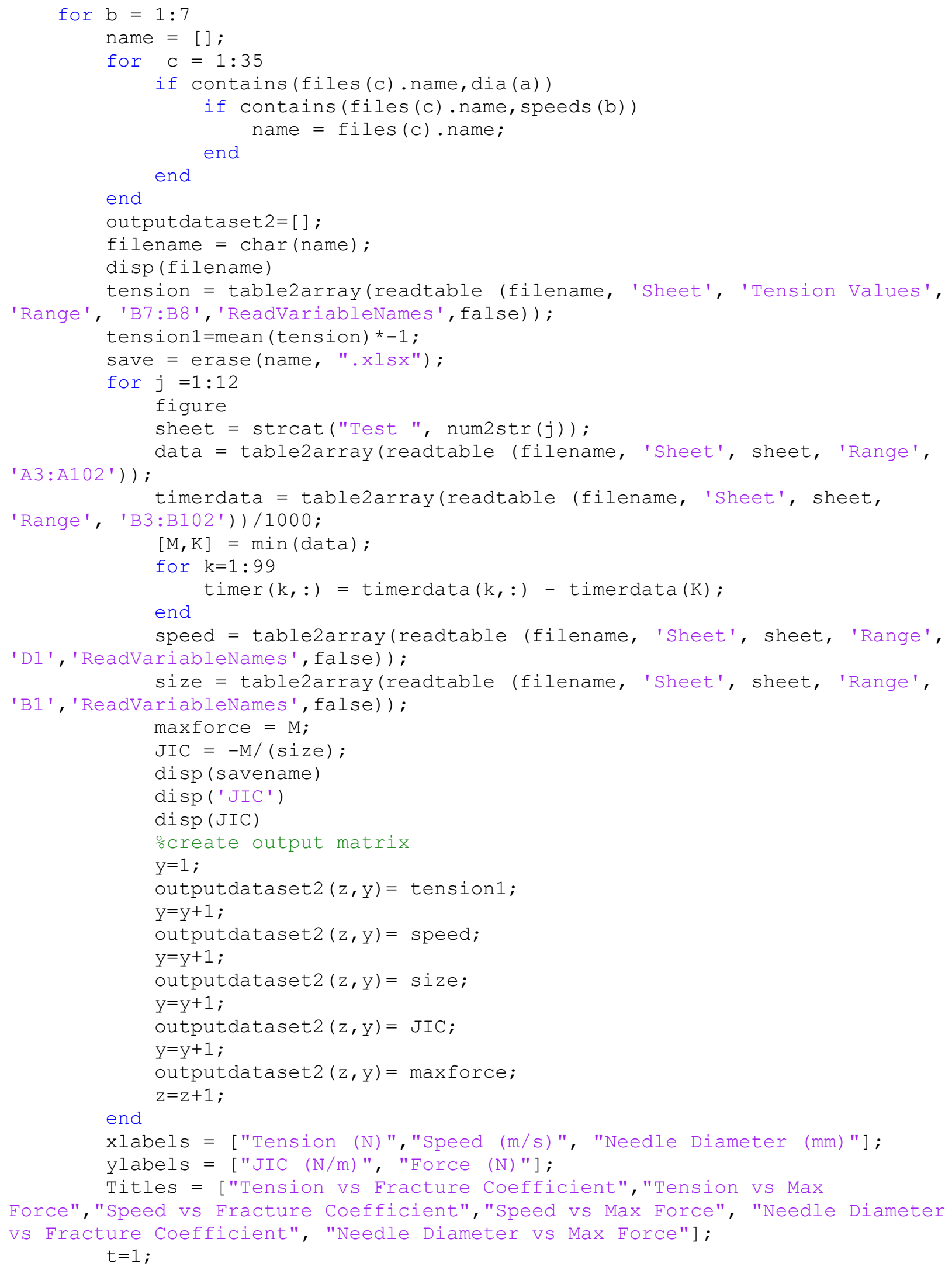




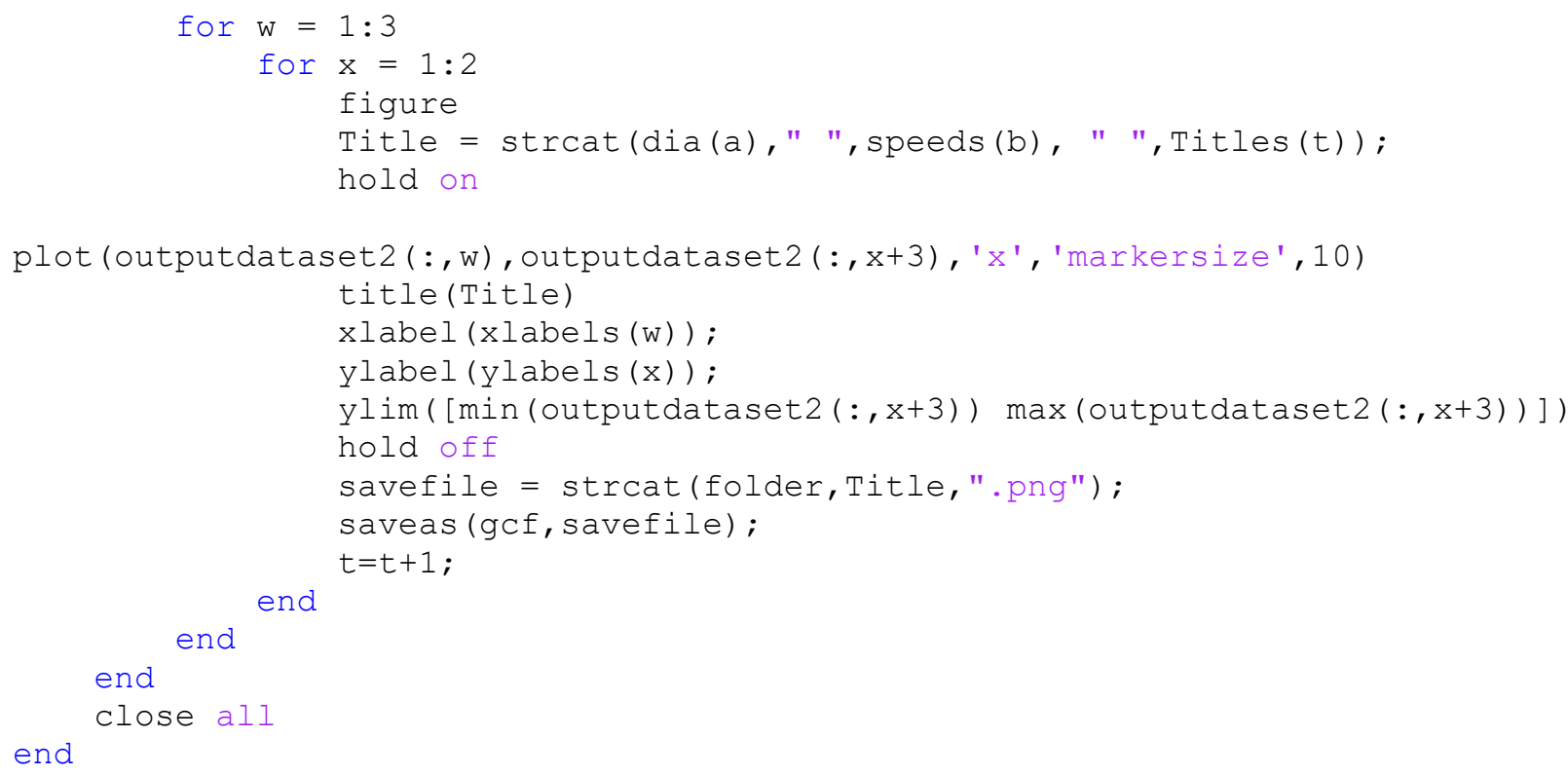




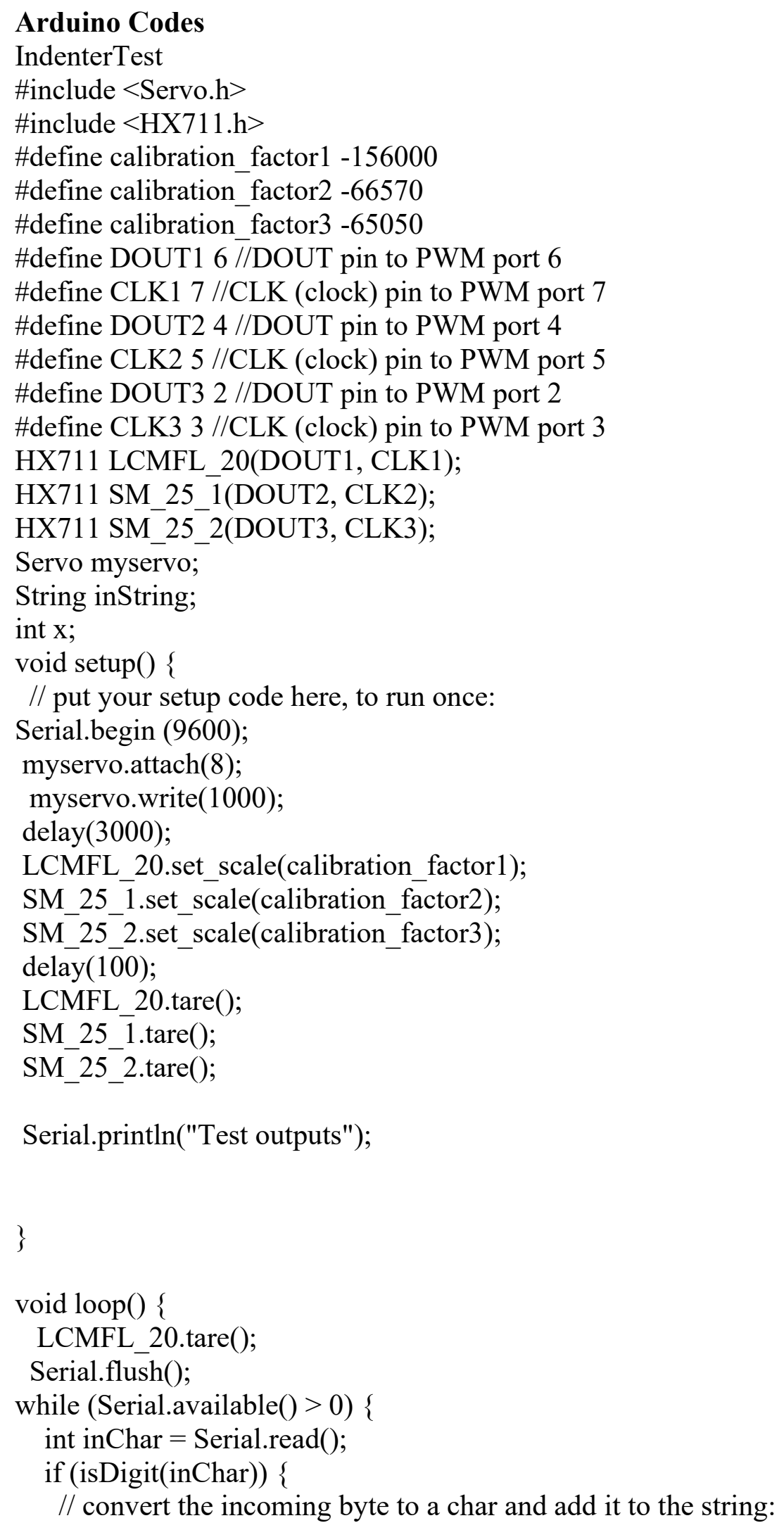




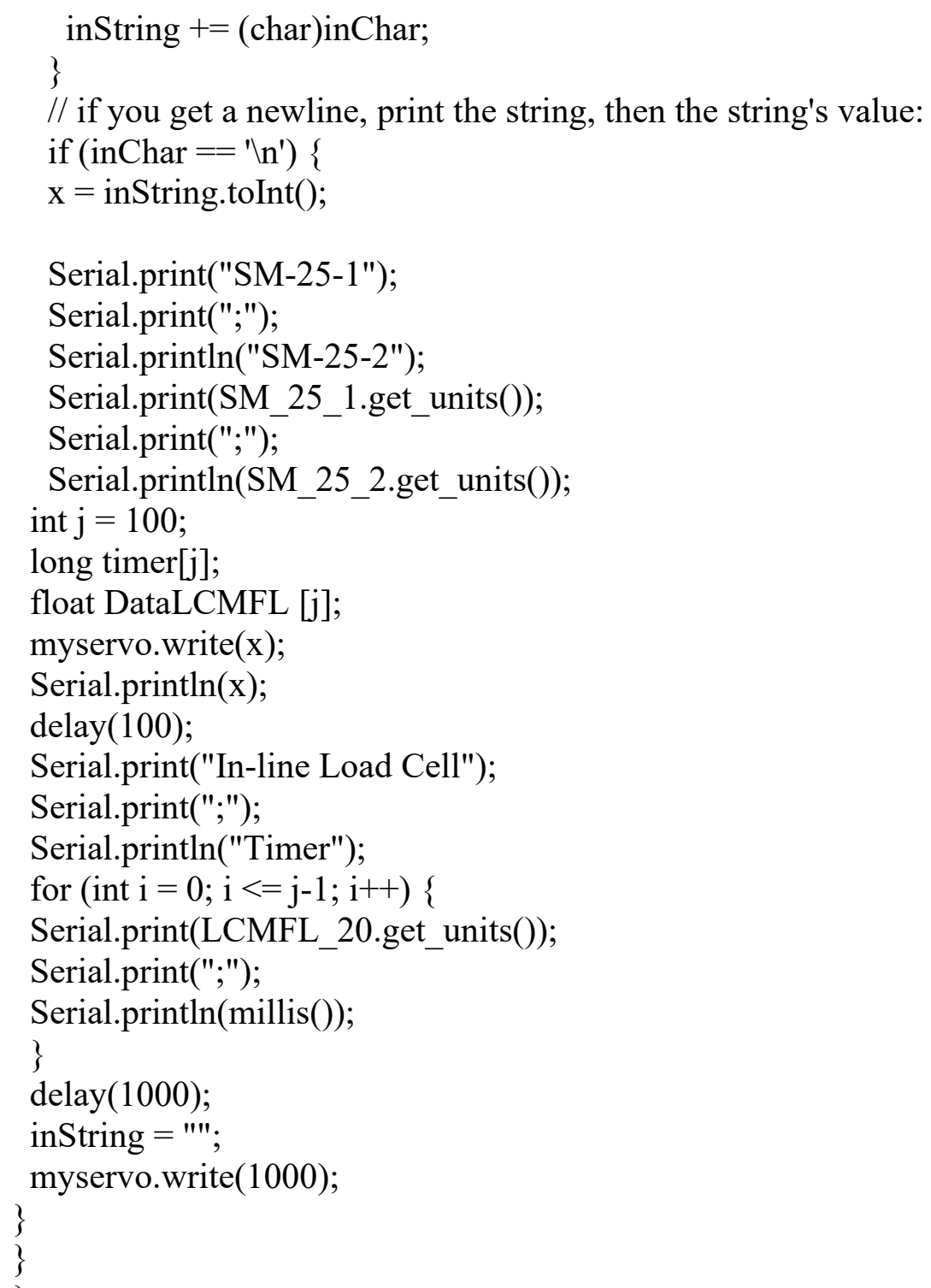


HX711Calibration

$/ *$

Example using the SparkFun HX711 breakout board with a scale

By: Nathan Seidle

SparkFun Electronics

Date: November 19th, 2014

License: This code is public domain but you buy me a beer if you use this and we meet someday (Beerware license).

This is the calibration sketch. Use it to determine the calibration_factor that the main example uses. It also

outputs the zero_factor useful for projects that have a permanent mass on the scale in between power cycles.

Setup your scale and start the sketch WITHOUT a weight on the scale

Once readings are displayed place the weight on the scale

Press $+/$ - or a/z to adjust the calibration_factor until the output readings match the known weight Use this calibration_factor on the example sketch

This example assumes pounds (lbs). If you prefer kilograms, change the Serial.print(" lbs"); line to $\mathrm{kg}$. The

calibration factor will be significantly different but it will be linearly related to lbs (1 lbs= $0.453592 \mathrm{~kg})$.

Your calibration factor may be very positive or very negative. It all depends on the setup of your scale system

and the direction the sensors deflect from zero state

This example code uses bogde's excellent library: https://github.com/bogde/HX711

bogde's library is released under a GNU GENERAL PUBLIC LICENSE

Arduino pin 2 -> HX711 CLK

$3->$ DOUT

$5 \mathrm{~V}->\mathrm{VCC}$

GND $->$ GND

Most any pin on the Arduino Uno will be compatible with DOUT/CLK.

The HX711 board can be powered from $2.7 \mathrm{~V}$ to $5 \mathrm{~V}$ so the Arduino $5 \mathrm{~V}$ power should be fine.

$* /$

\#include "HX711.h"

\#define DOUT 2

\#define CLK 3 
HX711 scale(DOUT, CLK);

float calibration_factor $=-65050 ; / /-7050$ worked for my $4401 \mathrm{~b}$ max scale setup

void $\operatorname{setup}()\{$

Serial.begin(9600);

Serial.println("HX711 calibration sketch");

Serial.println("Remove all weight from scale");

Serial.println("After readings begin, place known weight on scale");

Serial.println("Press + or a to increase calibration factor");

Serial.println("Press - or z to decrease calibration factor");

scale.set_scale();

delay (100);

scale.tare(); //Reset the scale to 0

long zero_factor = scale.read_average(); //Get a baseline reading

Serial.print("Zero factor: "); //This can be used to remove the need to tare the scale. Useful in permanent scale projects.

Serial.println(zero_factor);

\}

void $\operatorname{loop}()\{$

scale.set_scale(calibration_factor); //Adjust to this calibration factor

Serial.print("Reading: ");

Serial.print(scale.get_units(), 3);

Serial.print(" N"); //Change this to kg and re-adjust the calibration factor if you follow SI units like a sane person

Serial.print(" calibration_factor: ");

Serial.print(calibration_factor);

Serial.println();

delay(100);

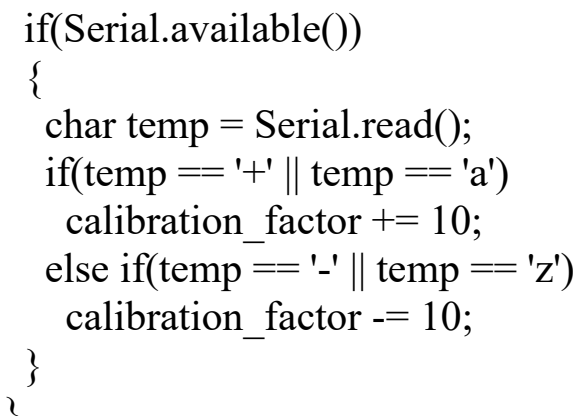




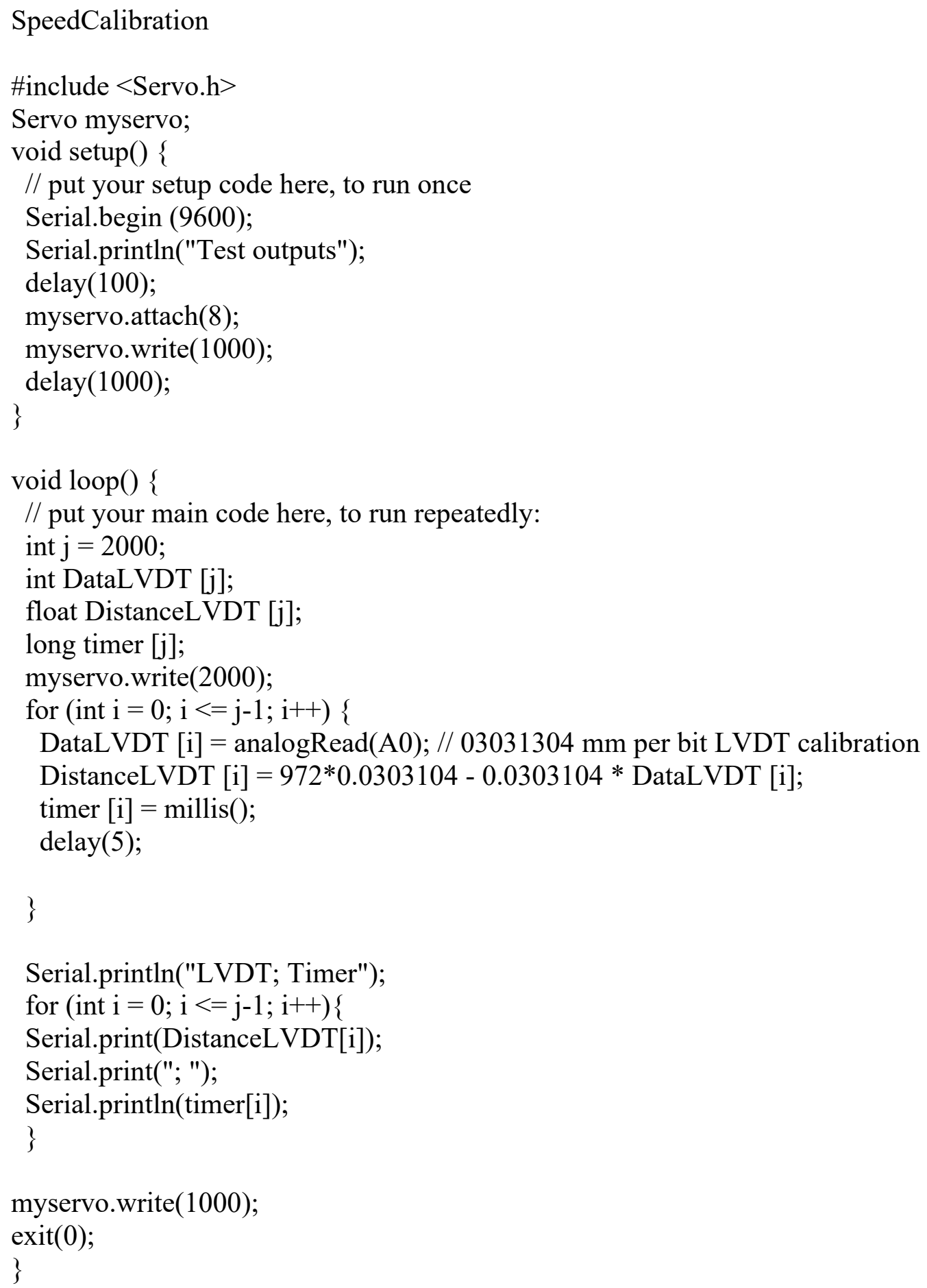




\section{Appendix E - Experimental Data Example}

This appendix gives an example of the raw data and the output that would be done using the Matlab codes from Appendix D.

Example Data Set: August 18 D2 1,23C 8S location1

\begin{tabular}{|c|c|c|c|c|}
\hline Test Location & $\begin{array}{l}\text { Quadrant } \\
1\end{array}$ & $\begin{array}{l}\text { Measured distance from tissue to } \\
\text { tip }\end{array}$ & 10 & \\
\hline $\begin{array}{l}\text { Displacement } \\
\text { set }\end{array}$ & 12.6 & $\begin{array}{l}\text { Approximate insertion } \\
\text { displacement }\end{array}$ & 2.6 & \\
\hline In-line Load Cell & Timer & Time & $\begin{array}{l}\text { Delta } \\
\mathrm{t}\end{array}$ & Position \\
\hline 0 & 588431 & 0 & & 0 \\
\hline 0 & 588521 & 90 & 90 & $\begin{array}{r}1.79312556 \\
8\end{array}$ \\
\hline 0 & 588611 & 180 & 90 & $\begin{array}{r}3.58625113 \\
6\end{array}$ \\
\hline-0.03 & 588701 & 270 & 90 & $\begin{array}{r}5.37937670 \\
5 \\
\end{array}$ \\
\hline-0.44 & 588791 & 360 & 90 & $\begin{array}{r}7.17250227 \\
3 \\
\end{array}$ \\
\hline-1.32 & 588881 & 450 & 90 & $\begin{array}{r}8.96562784 \\
1 \\
\end{array}$ \\
\hline-1.75 & 588971 & 540 & 90 & $\begin{array}{r}10.7587534 \\
1 \\
\end{array}$ \\
\hline-1.66 & 589061 & 630 & 90 & $\begin{array}{r}12.5518789 \\
8 \\
\end{array}$ \\
\hline-1.55 & 589151 & 720 & 90 & $\begin{array}{r}14.3450045 \\
5 \\
\end{array}$ \\
\hline-1.49 & 589241 & 810 & 90 & $\begin{array}{r}16.1381301 \\
1 \\
\end{array}$ \\
\hline-1.45 & 589331 & 900 & 90 & $\begin{array}{r}17.9312556 \\
8 \\
\end{array}$ \\
\hline-1.41 & 589421 & 990 & 90 & $\begin{array}{r}19.7243812 \\
5\end{array}$ \\
\hline-1.39 & 589511 & 1080 & 90 & $\begin{array}{r}21.5175068 \\
2 \\
\end{array}$ \\
\hline-1.37 & 589601 & 1170 & 90 & $\begin{array}{r}23.3106323 \\
9 \\
\end{array}$ \\
\hline-1.35 & 589691 & 1260 & 90 & $\begin{array}{r}25.1037579 \\
6 \\
\end{array}$ \\
\hline
\end{tabular}




\begin{tabular}{|c|c|c|c|c|}
\hline-1.33 & 589781 & 1350 & 90 & $\begin{array}{r}26.8968835 \\
2 \\
\end{array}$ \\
\hline-1.32 & 589871 & 1440 & 90 & $\begin{array}{r}28.6900090 \\
9 \\
\end{array}$ \\
\hline-1.3 & 589961 & 1530 & 90 & $\begin{array}{r}30.4831346 \\
6 \\
\end{array}$ \\
\hline-1.29 & 590051 & 1620 & 90 & $\begin{array}{r}32.2762602 \\
3\end{array}$ \\
\hline-1.28 & 590141 & 1710 & 90 & 34.0693858 \\
\hline-1.27 & 590231 & 1800 & 90 & $\begin{array}{r}35.8625113 \\
6 \\
\end{array}$ \\
\hline-1.26 & 590321 & 1890 & 90 & $\begin{array}{r}37.6556369 \\
3 \\
\end{array}$ \\
\hline-1.25 & 590411 & 1980 & 90 & 39.4487625 \\
\hline-1.25 & 590501 & 2070 & 90 & $\begin{array}{r}41.2418880 \\
7 \\
\end{array}$ \\
\hline-1.24 & 590591 & 2160 & 90 & $\begin{array}{r}43.0350136 \\
4 \\
\end{array}$ \\
\hline-1.23 & 590681 & 2250 & 90 & $\begin{array}{r}44.8281392 \\
1 \\
\end{array}$ \\
\hline-1.23 & 590771 & 2340 & 90 & $\begin{array}{r}46.6212647 \\
7 \\
\end{array}$ \\
\hline-1.22 & 590861 & 2430 & 90 & $\begin{array}{r}48.4143903 \\
4 \\
\end{array}$ \\
\hline-1.21 & 590951 & 2520 & 90 & $\begin{array}{r}50.2075159 \\
1 \\
\end{array}$ \\
\hline-1.21 & 591041 & 2610 & 90 & $\begin{array}{r}52.0006414 \\
8 \\
\end{array}$ \\
\hline-1.2 & 591131 & 2700 & 90 & $\begin{array}{r}53.7937670 \\
5 \\
\end{array}$ \\
\hline-1.2 & 591221 & 2790 & 90 & $\begin{array}{r}55.5868926 \\
2 \\
\end{array}$ \\
\hline-1.19 & 591311 & 2880 & 90 & $\begin{array}{r}57.3800181 \\
8 \\
\end{array}$ \\
\hline-1.19 & 591401 & 2970 & 90 & $\begin{array}{r}59.1731437 \\
5 \\
\end{array}$ \\
\hline-1.18 & 591491 & 3060 & 90 & $\begin{array}{r}60.9662693 \\
2 \\
\end{array}$ \\
\hline-1.18 & 591581 & 3150 & 90 & $\begin{array}{r}62.7593948 \\
9 \\
\end{array}$ \\
\hline-1.17 & 591671 & 3240 & 90 & $\begin{array}{r}64.5525204 \\
6 \\
\end{array}$ \\
\hline
\end{tabular}




\begin{tabular}{|c|c|c|c|c|}
\hline-1.17 & 591761 & 3330 & 90 & $\begin{array}{r}66.3456460 \\
2\end{array}$ \\
\hline-1.17 & 591851 & 3420 & 90 & $\begin{array}{r}68.1387715 \\
9 \\
\end{array}$ \\
\hline-1.16 & 591941 & 3510 & 90 & $\begin{array}{r}69.9318971 \\
6 \\
\end{array}$ \\
\hline-1.16 & 592031 & 3600 & 90 & $\begin{array}{r}71.7250227 \\
3\end{array}$ \\
\hline-1.16 & 592121 & 3690 & 90 & 73.5181483 \\
\hline-1.15 & 592211 & 3780 & 90 & $\begin{array}{r}75.3112738 \\
7 \\
\end{array}$ \\
\hline-1.15 & 592301 & 3870 & 90 & $\begin{array}{r}77.1043994 \\
3\end{array}$ \\
\hline-1.15 & 592391 & 3960 & 90 & 78.897525 \\
\hline-1.14 & 592481 & 4050 & 90 & $\begin{array}{r}80.6906505 \\
7 \\
\end{array}$ \\
\hline-1.14 & 592571 & 4140 & 90 & $\begin{array}{r}82.4837761 \\
4 \\
\end{array}$ \\
\hline-1.14 & 592661 & 4230 & 90 & $\begin{array}{r}84.2769017 \\
1 \\
\end{array}$ \\
\hline-1.13 & 592751 & 4320 & 90 & $\begin{array}{r}86.0700272 \\
7 \\
\end{array}$ \\
\hline-1.13 & 592841 & 4410 & 90 & $\begin{array}{r}87.8631528 \\
4 \\
\end{array}$ \\
\hline-1.13 & 592931 & 4500 & 90 & $\begin{array}{r}89.6562784 \\
1 \\
\end{array}$ \\
\hline-1.12 & 593021 & 4590 & 90 & $\begin{array}{r}91.4494039 \\
8\end{array}$ \\
\hline-1.12 & 593111 & 4680 & 90 & $\begin{array}{r}93.2425295 \\
5 \\
\end{array}$ \\
\hline-1.12 & 593201 & 4770 & 90 & $\begin{array}{r}95.0356551 \\
2 \\
\end{array}$ \\
\hline-1.12 & 593291 & 4860 & 90 & $\begin{array}{r}96.8287806 \\
8 \\
\end{array}$ \\
\hline-1.11 & 593381 & 4950 & 90 & $\begin{array}{r}98.6219062 \\
5 \\
\end{array}$ \\
\hline-1.11 & 593471 & 5040 & 90 & $\begin{array}{r}100.415031 \\
8 \\
\end{array}$ \\
\hline-1.11 & 593561 & 5130 & 90 & $\begin{array}{r}102.208157 \\
4\end{array}$ \\
\hline-1.11 & 593651 & 5220 & 90 & 104.001283 \\
\hline-1.1 & 593741 & 5310 & 90 & 105.794408 \\
\hline
\end{tabular}




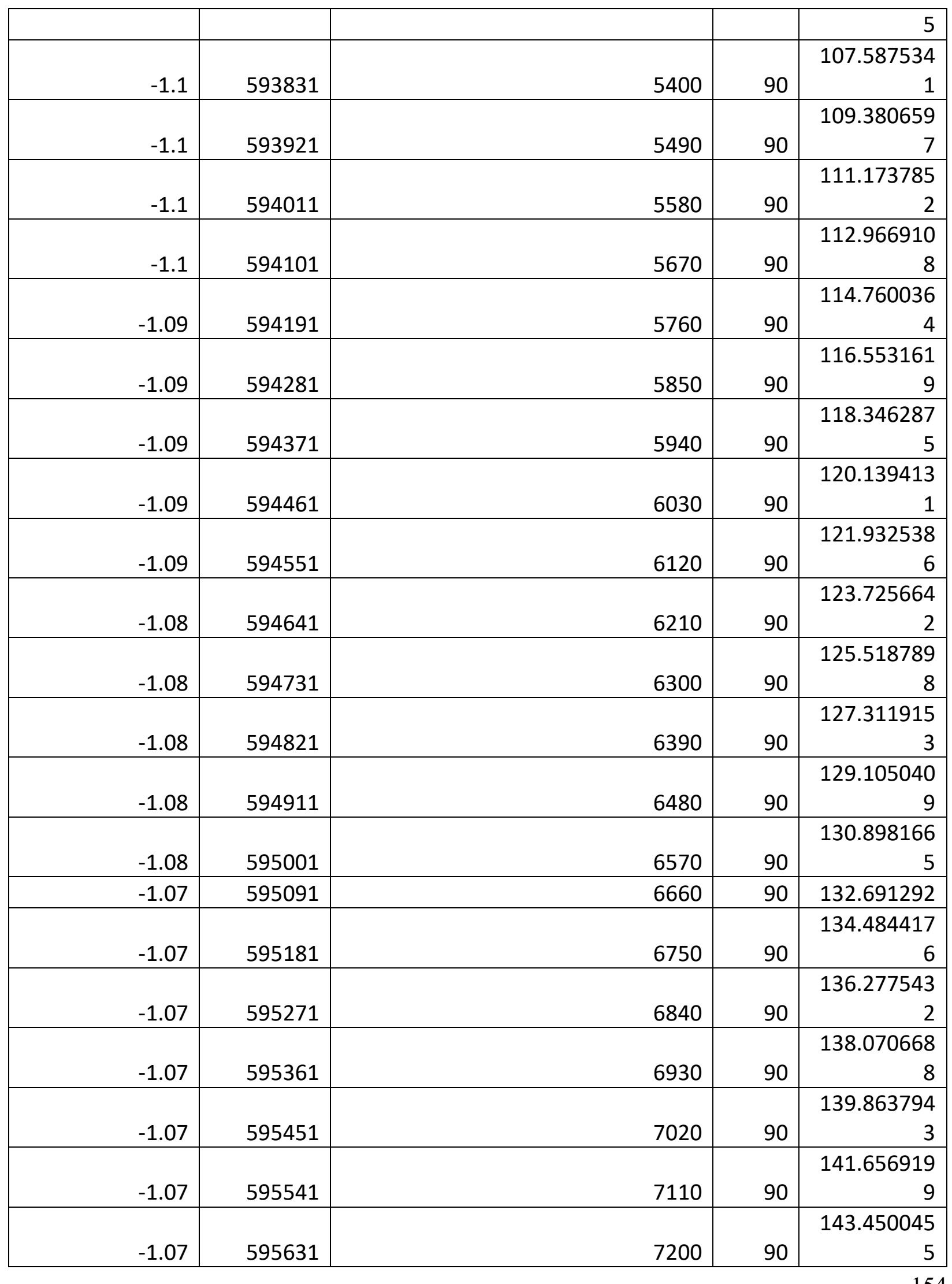




\begin{tabular}{|c|c|c|c|c|}
\hline-1.06 & 595721 & 7290 & 90 & 145.243171 \\
\hline & & & & 147.036296 \\
\hline-1.06 & 595811 & 7380 & 90 & 6 \\
\hline & & & & 148.829422 \\
\hline-1.06 & 595901 & 7470 & 90 & 2 \\
\hline & & & & 150.602624 \\
\hline-1.06 & 595990 & 7559 & 89 & 1 \\
\hline & & & & 152.395749 \\
\hline-1.06 & 596080 & 7649 & 90 & 7 \\
\hline & & & & 154.188875 \\
\hline-1.05 & 596170 & 7739 & 90 & 3 \\
\hline & & & & 156.001924 \\
\hline-1.05 & 596261 & 7830 & 91 & 4 \\
\hline-1.05 & 596351 & 7920 & 90 & 157.79505 \\
\hline & & & & 159.588175 \\
\hline-1.05 & 596441 & 8010 & 90 & 6 \\
\hline & & & & 161.381301 \\
\hline-1.05 & 596531 & 8100 & 90 & 1 \\
\hline & & & & 163.174426 \\
\hline-1.05 & 596621 & 8190 & 90 & 7 \\
\hline & & & & 164.967552 \\
\hline-1.05 & 596711 & 8280 & 90 & 3 \\
\hline & & & & 166.760677 \\
\hline-1.05 & 596801 & 8370 & 90 & 8 \\
\hline & & & & 168.553803 \\
\hline-1.04 & 596891 & 8460 & 90 & 4 \\
\hline-1.04 & 596981 & 8550 & 90 & 170.346929 \\
\hline & & & & 172.140054 \\
\hline-1.04 & 597071 & 8640 & 90 & 5 \\
\hline
\end{tabular}




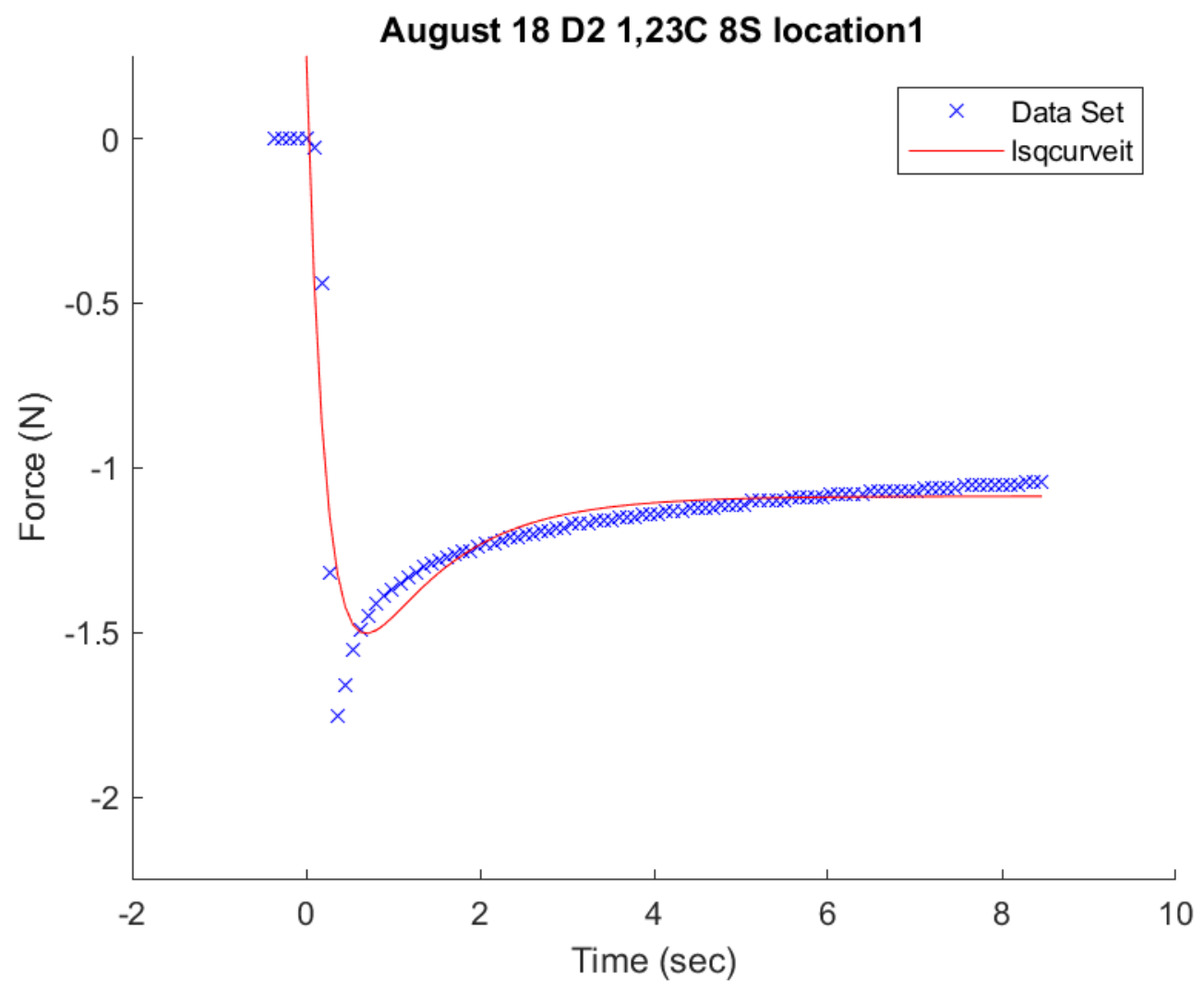




\section{Appendix F - Instrumentation Data Sheets}

This appendix gives the manufacture's data sheets of all instrumentation used. 


\section{ECONOMICAL MINIATURE TENSION OR COMPRESSION LOAD CELLS 19 TO 25 mm DIAMETER}

\section{Tension/Compression Calibrated in Tension 0-10 to 0-500 N}

\section{LCMFL Series}

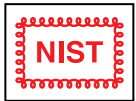

Small and capable of highly accurate readings, the LCMFL subminiature load cells are suitable for precision industrial applications. They are all stainless steel, measure either tension or compression loads, and have male thread studs for load attachment. The exclusive internal design provides superior long-term stability and minimizes the effects of small off-axis loads.

\section{SPECIFICATIONS}

\section{Excitation:}

10 to $50 \mathrm{~N}: 5 \mathrm{Vdc}$

$\geq 100 \mathrm{~N}: 10 \mathrm{Vdc}$

Output:

$10 \mathrm{~N}: 1.0 \mathrm{mV} / \mathrm{V}$ (nom.)

20 to $500 \mathrm{~N}: 2 \mathrm{mV} / \mathrm{V}$ (nom.)

Accuracy (Linearity and

Hysteresis Combined): $\pm 0.50 \%$ FSO

Repeatability: $\pm 0.20 \%$ FSO

5-Point Calibration (in Tension):

0\%, 50\%, 100\%, 50\%, 0\%

Zero Balance: $\pm 2 \%$ FSO

Operating Temp Range:

-53 to $121^{\circ} \mathrm{C}\left(-65\right.$ to $\left.250^{\circ} \mathrm{F}\right)$

Compensated Temp Range:

16 to $71^{\circ} \mathrm{C}\left(60\right.$ to $\left.160^{\circ} \mathrm{F}\right)$

Thermal Effects:

Span: $\geq 1 \mathrm{~kg}: \pm 0.009 \% \mathrm{rdg} /{ }^{\circ} \mathrm{C}$

Zero: $\geq 1 \mathrm{~kg}: 0.009 \% \mathrm{FSO} /{ }^{\circ} \mathrm{C}$

Deflection FS: 0.03 to $0.08 \mathrm{~mm}$ (0.001 to $0.003 ")$

Safe Overload: $150 \%$ of capacity Ultimate Overload: $300 \%$ of capacity Bridge Resistance: $350 \Omega$ minimum

Construction: Stainless steel

Electrical Connection: $1.5 \mathrm{~m}\left(5^{\prime}\right)$

4-conductor, shielded TFE cable

Protection Rating: IP54
LCMFL-50N, shown larger than actual size.
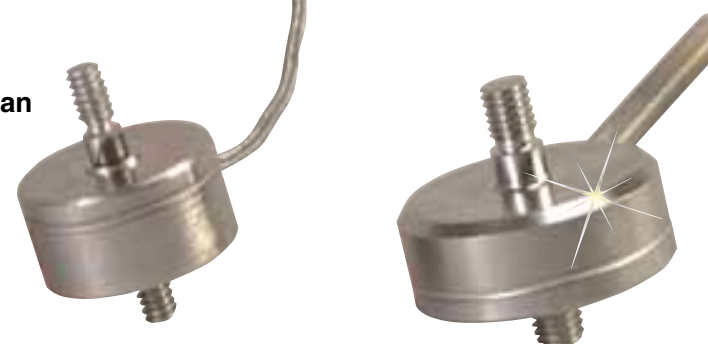

LCMFL-500N shown larger than actual size.

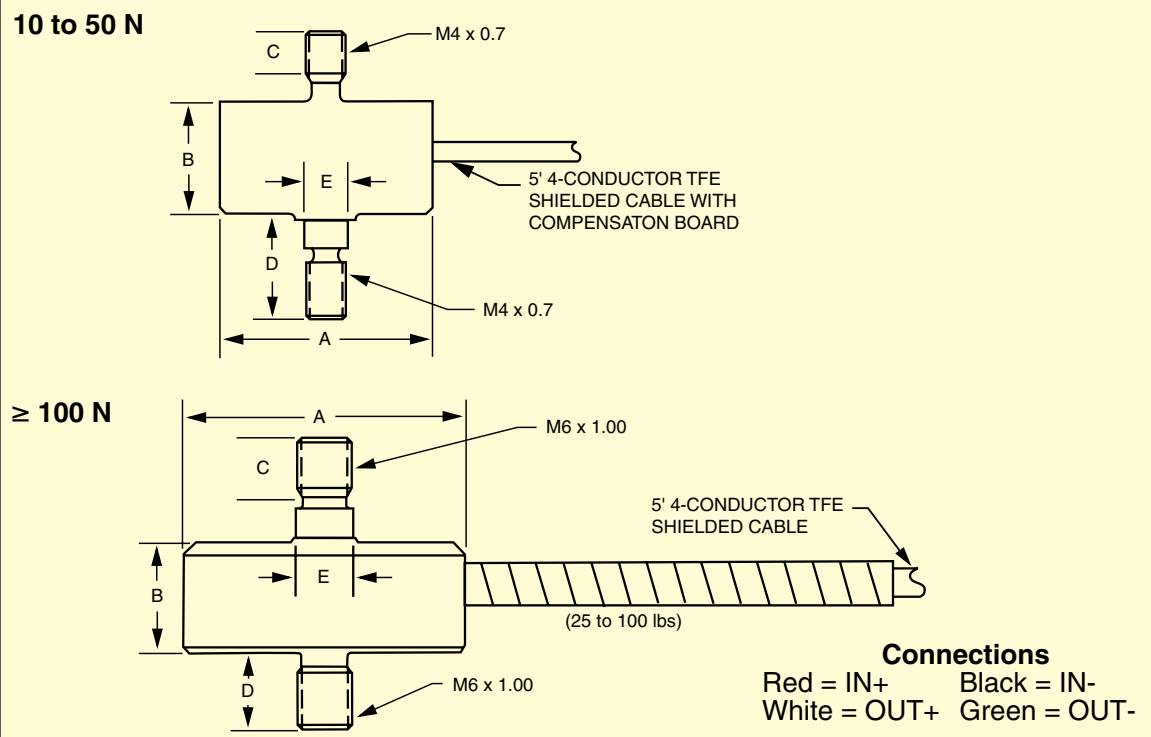

Dimensions: $\mathrm{mm}$ (inch)

\begin{tabular}{|l|c|c|c|c|c|}
\hline CAPACITY & A & B & C & D & E \\
\hline 10 to $50 \mathrm{~N}$ & $19.1(0.75)$ & $10.1(0.40)$ & $6.4(0.25)$ & $9.7(0.38)$ & $3.8(0.15)$ \\
\hline$\geq 100 \mathrm{~N}$ & $25.4(1.00)$ & $9.9(0.39)$ & $9.7(0.38)$ & $7.0(0.26)$ & $5.0(0.20)$ \\
\hline
\end{tabular}

\begin{tabular}{|l|l|l|l|}
\hline \multicolumn{2}{|l|}{ To Order } \\
\cline { 1 - 2 } \multicolumn{2}{|c|}{ CAPACITY } & \multirow{2}{*}{ MODEL NO. } & COMPATIBLE METERS* \\
\cline { 1 - 2 } N & lb & MPE- \\
\hline 10 & 2.2 & LCMFL-10N & DP25B-S, DP41-S, DPiS \\
\hline 20 & 4.5 & LCMFL-20N & DP25B-S, DP41-S, DPiS \\
\hline 50 & 11 & LCMFL-50N & DP25B-S, DP41-S, DPiS \\
\hline 100 & 22 & LCMFL-100N & DP25B-S, DP41-S, DPiS \\
\hline 200 & 45 & LCMFL-200N & DP25B-S, DP41-S, DPiS \\
\hline 500 & 112 & LCMFL-500N & DP25B-S, DP41-S, DPiS \\
\hline
\end{tabular}

Comes with 5-point NIST-traceable calibration.

* DPiS meter suitable for one direction measurement only.

Ordering Examples: LCMFL-10N, $10 \mathrm{~N}$ load cell, MREC-M4F, matching rod end. LCMFL-200N, $200 \mathrm{~N}$ load cell, MREC-M6F, matching rod end.

ACCESSORIES

\begin{tabular}{|l|l|}
\hline MODEL NO. & DESCRIPTION \\
\hline MREC-M4F & M4 x 0.7 female-thread rod end \\
\hline MREC-M6F & M6 $\times 1.00$ female-thread rod end \\
\hline
\end{tabular}




\section{Model SM S-Type Load Cell (U.S. \& Metric)}

Why the Interface model SM S-Type Load Cell is the best in class:

- Proprietary Interface temperature compensated strain gages

- High performance

- $.0008 \% /{ }^{\circ} \mathrm{F}\left(.0015 \% /{ }^{\circ} \mathrm{C}\right)$ temp. effect on output

- Lowest creep - 0.025\%

- Tension and compression

\section{STANDARD CONFIGURATION}

$5 \mathrm{ft}$ Integral Cable (SM-nn)

\section{OPTIONS}

Extra Cable Length

Standardized Output

\section{ACCESSORIES}

Load Button

Mounting Hardware

Instrumentation

Consult factory for more technical information
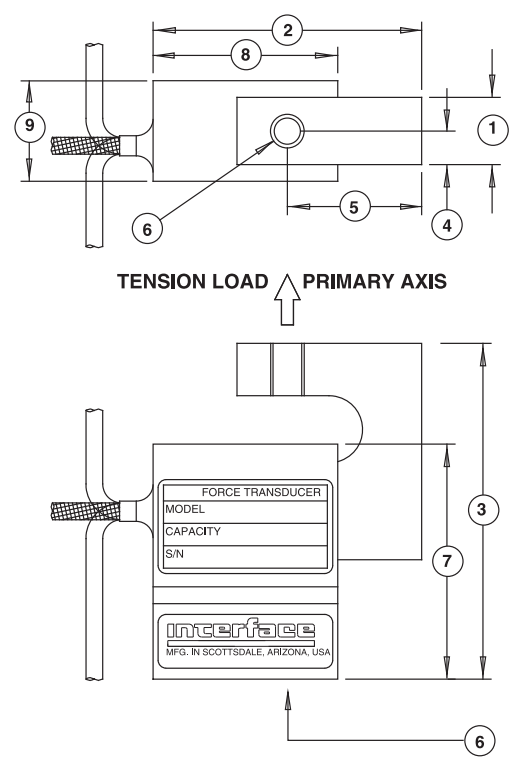

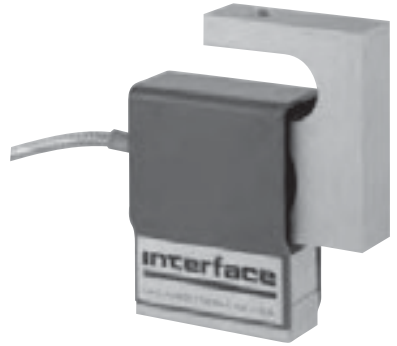

\section{SPECIFICATIONS}

ACCURACY - (MAX ERROR)

Nonlinearity-\% FS .......................... \pm 0.03

Hysteresis-\% FS …............................ \pm 0.02

Nonrepeatability-\% R0 …................... \pm 0.01

Creep, in 20 min-\% ............................... \pm 0.025

TEMPERATURE

Compensated Range $-{ }^{\circ} \mathrm{F}$....................... 0 to 150

Compensated Range- ${ }^{\circ} \mathrm{C}$.......................-15 to 65

Operating Range- ${ }^{\circ} \mathrm{F}$................................... -65 to 200

Operating Range- ${ }^{\circ} \mathrm{C}$...................................... 55 to 90

Effect on Output- $\% / F-M A X \ldots \ldots \ldots . . . . . \pm 0.0008$

Effect on Output $\% /{ }^{\circ} \mathrm{C}-$ MAX ................ \pm 0.0015

Effect on Zero- $\%$ RO/ $/ F-M A X \ldots \ldots \ldots . . . . \pm 0.0015$

Effect on Zero- \% RO/ $\mathrm{C}-$ MAX ............... \pm 0.0027

\section{ELECTRICAL}

Rated Output-mV/N (Nominal) ...........3.0

Zero Balance-\% RO ........................... \pm 1.0

Bridge Resistance-Ohm (Nominal) ....350

Excitation Voltage - MAX .....................15 VDC

Insulation Resistance-Megohm .......... 5000

\section{MECHANICAL}

Calibration .........................................Tension

Safe Overload-\% CAP ...................... \pm 150

Cable length-ft ................................. 5

Natural Frequency/Deflection:

\begin{tabular}{rrcc} 
Ibf & N & $\begin{array}{c}\text { Deflection } \\
\text { (inches) }\end{array}$ & $\begin{array}{c}\text { Nat. Freq. } \\
\text { (Hertz) }\end{array}$ \\
10 & 50 & .003 & 600 \\
25 & 100 & .003 & 1000 \\
50 & 200 & .003 & 1550 \\
100 & 500 & .004 & 1850 \\
250 & 1000 & .006 & 2350 \\
500 & 2000 & .006 & 2150 \\
1000 & 5000 & .005 & 3350 \\
\hline
\end{tabular}

\section{DIMENSIONS}

\begin{tabular}{|c|c|c|c|c|}
\hline \multirow{4}{*}{$\begin{array}{c}\text { See } \\
\text { Drawing }\end{array}$} & \multicolumn{4}{|c|}{ CAPACITY } \\
\hline & U.S. (Ibf) & Metric (N) & U.S. (lbf) & Metric (N) \\
\hline & $10,25,50$ & $50,100,200$ & 500 & 2000 \\
\hline & $100,150,250$ & 500,1000 & 1000 & 5000 \\
\hline & inch & $\mathrm{mm}$ & inch & $\mathrm{mm}$ \\
\hline (1) & 0.50 & 12.7 & 1.00 & 25.4 \\
\hline (2) & 2.00 & 50.8 & 2.00 & 50.8 \\
\hline (3) & 2.50 & 63.5 & 3.00 & 76.2 \\
\hline (4) & 0.25 & 6.40 & 0.50 & 12.7 \\
\hline (5) & 1.00 & 25.4 & 1.00 & 25.4 \\
\hline (6) & 1/4-28 UNF-2B & $M 6 \times 1-6 \mathrm{H}$ & 1/2-20 UNF-2B & M12x1.75-6H \\
\hline (7) & 1.75 & 44.5 & 2.00 & 50.8 \\
\hline (8) & 1.38 & 35.1 & 1.94 & 49.3 \\
\hline (9) & 0.75 & 19.1 & 1.25 & 31.8 \\
\hline
\end{tabular}




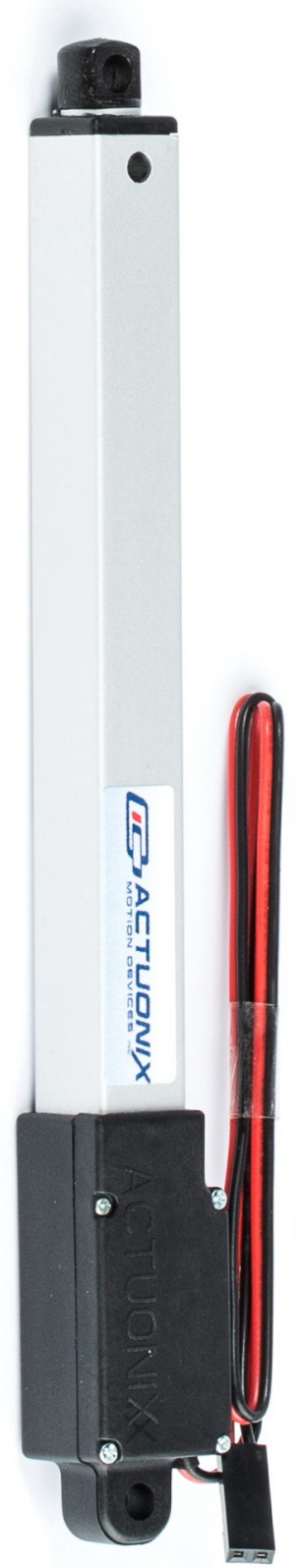

100mm L12 Actuator Actual Size

\section{Benefits}

$\rightarrow$ Compact

$\rightarrow$ Simple control

$\rightarrow$ Low voltage

$\rightarrow$ Equal push/pull

$\rightarrow$ Easy mounting

Applications

$\rightarrow$ Robotics

$\rightarrow$ Appliances

$\rightarrow$ Toys

$\rightarrow$ RC vehicles

$\rightarrow$ Automotive

$\rightarrow$ Industrial Automation

\section{Miniature Linear Motion Series · L12}

Actuonix Motion Devices unique line of Miniature Linear Actuators enables a new generation of motion-enabled product designs, with capabilities that have never before been combined in a device of this size. These small linear actuators are a superior alternative to designing with awkward gears, motors, servos, and linkages.

Actuonix's $L$ series of micro linear actuators combine the best features of our existing micro actuator families into a highly flexible, configurable, and compact platform with an optional sophisticated on-board microcontroller. The first member of the $L$ series, the L12, is an axial design with a powerful drive-train and a rectangular cross section for increased rigidity. But by far the most attractive feature of this actuator is the broad spectrum of available configurations.

\section{L12 Specifications}

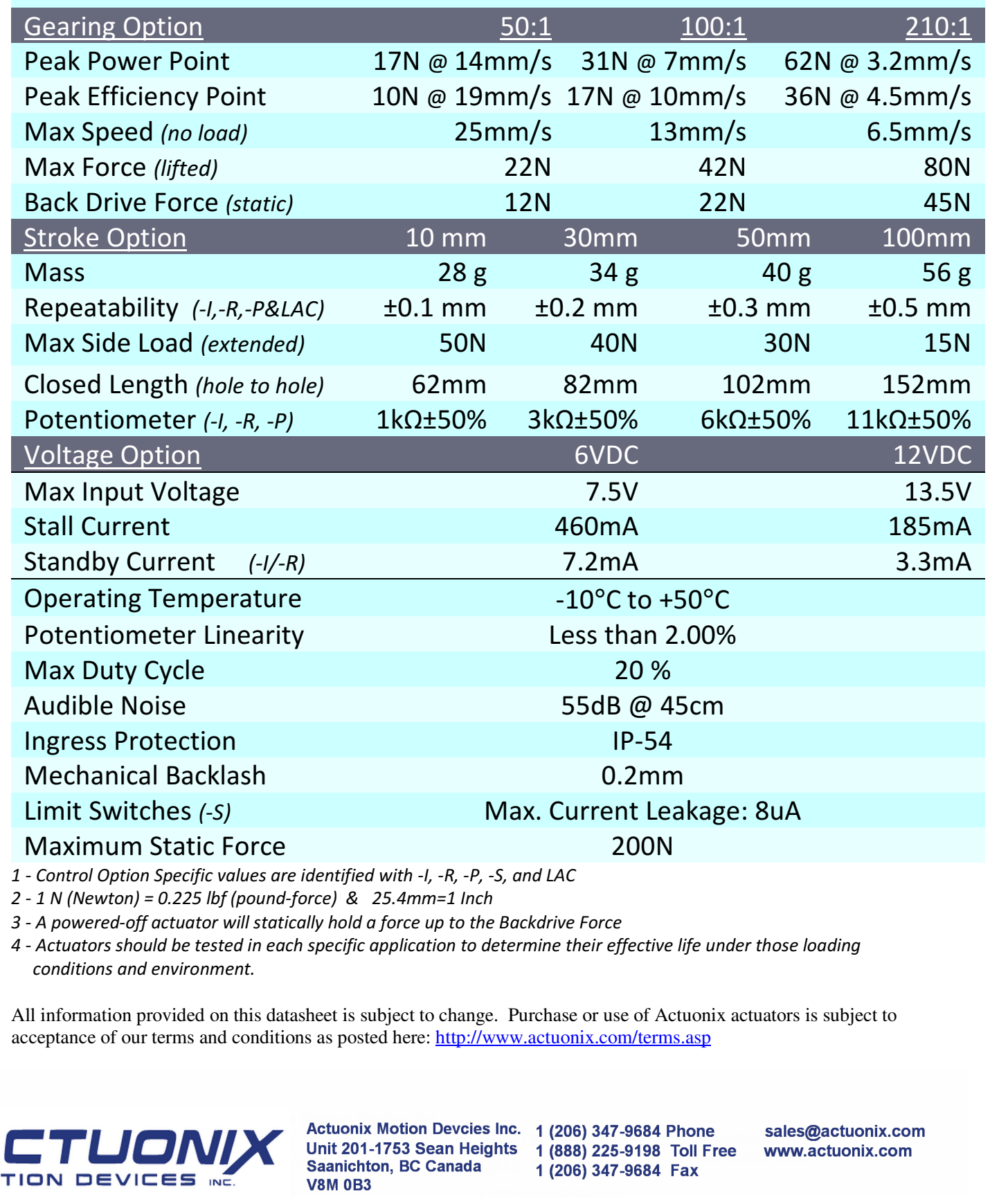




\section{L12 Specifications}
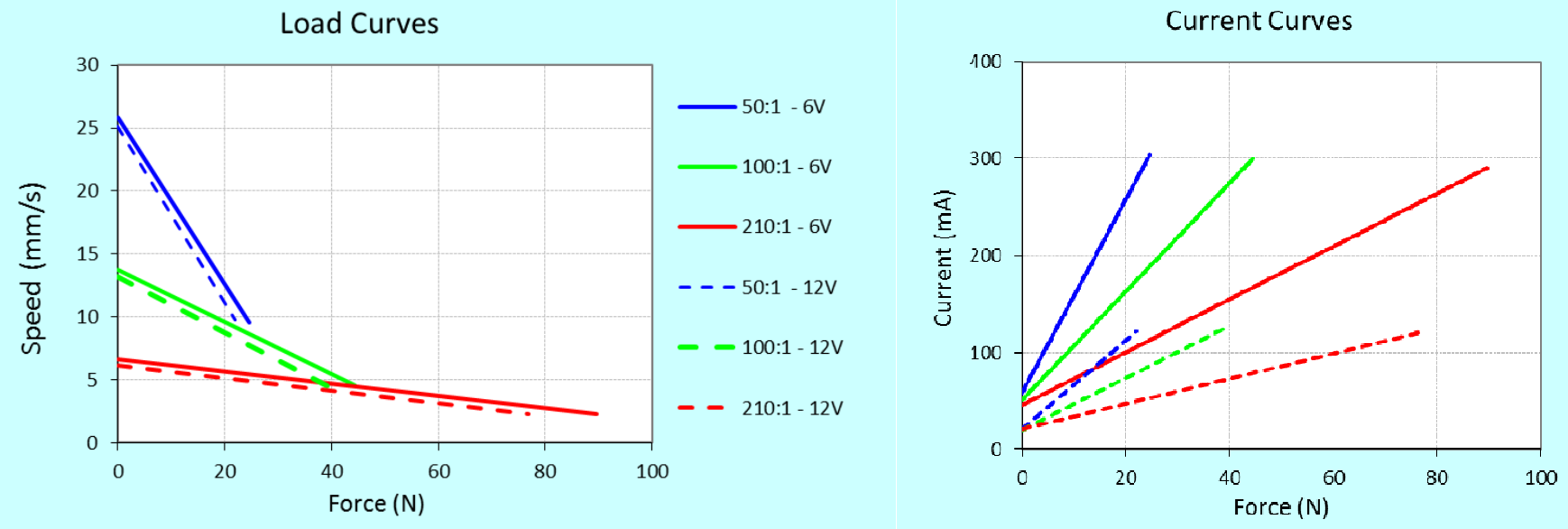

\section{Model Selection}

L12 options are identified according to the following scheme:

\section{L12-SS-GG-VV-C}

\begin{tabular}{|c|c|}
\hline feature & Options \\
\hline SS: Stroke Length & $10,30,50,100$ \\
\hline $\begin{array}{l}\text { GG: Gear reduction } \\
\text { ratio (refer to load } \\
\text { curves above) }\end{array}$ & $\begin{array}{l}\mathbf{5 0}, \mathbf{1 0 0}, \mathbf{2 1 0} \\
\text { (lower ratios are faster but push } \\
\text { less force, and vice versa) }\end{array}$ \\
\hline VV: Voltage & $\begin{array}{l}6,12 \\
\text { (DC volts) }\end{array}$ \\
\hline C: Controller & $\begin{array}{l}\text { S Limit Switches } \\
\text { P Potentiometer Feedback } \\
\text { I Integrated Controller } \\
\text { R RC Servo Integrated Controller }\end{array}$ \\
\hline
\end{tabular}

\section{L12 Controller Options}

\section{Option S - End of Stroke Limit Switches}

WIRING: (see last page for pin numbering)

1 - Red - Motor V+

2 - Black - Motor V- (Gnd)

The $-S$ actuators have limit switches that will turn off power to the motor when the actuator reaches within $0.5 \mathrm{~mm}$ of the end of stroke. Internal diodes allow the actuator to reverse away from the limit switch. The limit switches cannot be moved once the actuator is manufactured. While voltage is applied to the motor power pins, $(1 \& 2)$ the actuator extends. Reverse the polarity and the actuator retracts. This can be accomplished manually with a DPDT switch or relay, or using an $\mathrm{H}$-Bridge circuit. The $-\mathrm{S}$ model cannot be used with the LAC control board.

\section{Option P - Potentiometer Position Feedback}

WIRING: (see last page for pin numbering)

1 - Orange - Feedback Potentiometer negative reference rail

2 - Purple - Feedback Potentiometer wiper

3 - Red - Motor V+ (6V or 12V)

4 - Black - Motor V- (Ground)

5 - Yellow - Feedback Potentiometer positive reference rail

The -P actuators have no built in controller, but do provide an analog position feedback signal that can be input to an external controller. While voltage is applied to the motor power pins, ( $3 \& 4$ ) the actuator extends. Reverse the polarity and the actuator retracts. This can be accomplished manually with a DPDT switch or relay, or using an H-Bridge circuit. Position of the actuator stroke can be monitored by providing any stable low and high reference voltage on pins $1 \& 5$, then reading the position signal on pin 2 . The voltage on pin 2 will vary linearly between the two reference voltages in proportion to the position of the actuator stroke.

The L12 -P actuator can be used as a linear servo by connecting the actuator to an external controller such as the LAC board offered by Firgelli. This control board reads the position signal from the L12, compares it with your input control signal then commands the actuator to move via an onboard $\mathrm{H}$-bridge circuit. The LAC allows any one of the following control inputs: Analog 0-5V or 4-20mA, or Digital 05V PWM, 1-2ms Standard RC, or USB. The RC input effectively transforms your L12 into a linear servo, which is a direct replacement for any common hobby servo used in RC toys and robotics. Refer to the LAC datasheet for more details. 
Option I - Integrated controller with industrial and RC servo interfaces

1 - Green - Current input signal (4-20 mA interface mode)

2 - Blue - Voltage input signal (0-5V interface mode)

3 - Purple - Position Feedback signal (Proportional 0-3.3 V)

4 - White - RC input signal (RC-servo compatible mode)

5 - Red - Power (6V model or $12 \mathrm{~V}$ model)

6 - Black - Ground

The -1 actuator models feature an on-board software-based digital position controller. The controller is not userprogrammable. The six lead wires are split into two connectors. Leads 4,5 and 6 terminate at a universal RC servo three-pin connector ( $\mathrm{Hi}-\mathrm{Tec}^{\mathrm{TM}}$ and Futaba ${ }^{\mathrm{TM}}$ compatible). Leads 1,2 , and 3 terminate at a separate, similarly sized connector.

When the actuator is powered up, it will repeatedly scan leads $1,2,4$ for an input signal that is valid under any of the four supported interface modes. When a valid signal is detected, the actuator will self-configure to the corresponding interface mode, and all other interface modes and input leads are disabled until the actuator is next powered on.

0-5 V Interface Mode: This mode allows the actuator to be controlled with just a battery, and a potentiometer to signal the desired position to the actuator - a simple interface for prototypes or home automation projects. The desired actuator position (set point) is input to the actuator on lead 2 as a voltage between ground and $5 \mathrm{~V}$. The set point voltage must be held on lead 2 even when the desired actuator stroke position is reached. Lead 2 is a high impedance input.

4-20 mA Interface Mode: This mode is compatible with PLC devices typically used in industrial control applications. The desired actuator position (set point) is input to the actuator on lead 1 as a current between $4 \mathrm{~mA}$ and $20 \mathrm{~mA}$. The set point current must be held on lead 1 even when the desired actuator stroke position is reached.

RC Servo Interface Mode: This is a standard hobby-type remote-control digital servo interface (CMOS logic), compatible with servos and receivers from manufacturers like Futaba $^{\mathrm{TM}}$ and $\mathrm{Hi}-\mathrm{Tec}^{\mathrm{TM}}$. The desired actuator position is input to the actuator on lead 4 as a positive 5 Volt pulse width signal. A 1.0 ms pulse commands the controller to fully retract the actuator, and a $2.0 \mathrm{~ms}$ pulse signals full extension. If the motion of the actuator, or of other servos in your system, seems erratic, place a $1-4 \Omega$ resistor in series with the actuator's red $\mathrm{V}+$ lead wire.

PWM Mode: This mode allows control of the actuator using a single digital output pin from an external microcontroller. The desired actuator position is encoded as the duty cycle of a 5 Volt $1 \mathrm{kHz}$ square wave on actuator lead 2, where the \% duty cycle sets the actuator position to the same \% of full stroke extension. The waveform must be $0 \mathrm{~V}$ to $+5 \mathrm{~V}$ in order to access the full stroke range of the actuator.
Option R - RC Linear Servo

WIRING: (see last page for pin numbering)

1 - White - RC input signal (RC-servo compatible)

2 - Red - Power (+6 VDC)

3 - Black - Ground

Note: Reversing the polarity of pins 2 and 3 may permanently damage the actuator

The - $\mathrm{R}$ actuators or 'linear servos' are a direct replacement for regular radio controlled hobby servos. The desired actuator position is input to the actuator on lead 1 as a positive 5 Volt pulse width signal. A $1.0 \mathrm{~ms}$ pulse commands the controller to fully retract the actuator, and a $2.0 \mathrm{~ms}$ pulse signals it to fully extend. If the motion of the actuator, or of other servos in your system, seems erratic, place a $1-4 \Omega$ resistor in series with the actuator's red V+ lead wire.

The $-R$ actuators are available in 6 volt and 30, 50 and $100 \mathrm{~mm}$ strokes only.

This is a lower cost option for the RC hobby market. While the supported control options are reduced, the $-\mathrm{R}$ retains the same great precision and cycle life as the $-I$.

\section{Ordering}

Small quantity orders can be placed directly online at www.actuonix.com. Purchase orders, volume quotes, and custom requests can be sent to sales@actuonix.com .

Each actuator ships with two mounting brackets and \#8-32 mounting hardware. The cable length is approximately $300 \mathrm{~mm}$ and connector is a $0.1^{\prime \prime}$ pitch female socket connector.

\section{Custom Options}

Contact Actuonix for quotes and lead-times on any nonstandard customizations. Minimum order quantity for customizations is 500 pieces.

Any modifications requiring mold changes will involve higher MOQ and/or NRE fees.

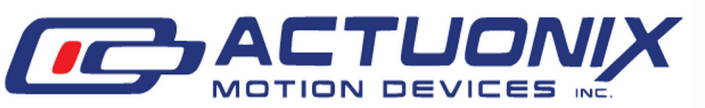

MOTIDN DEVICES INC
Actuonix Motion Devcies Inc. 1 (206) 347-9684 Phone Unit 201-1753 Sean Heights 1 (888) 225-9198 Toll Free Saanichton, BC Canada 1 (206) 347-9684 Fax V8M 0B3 sales@actuonix.com www.actuonix.com 


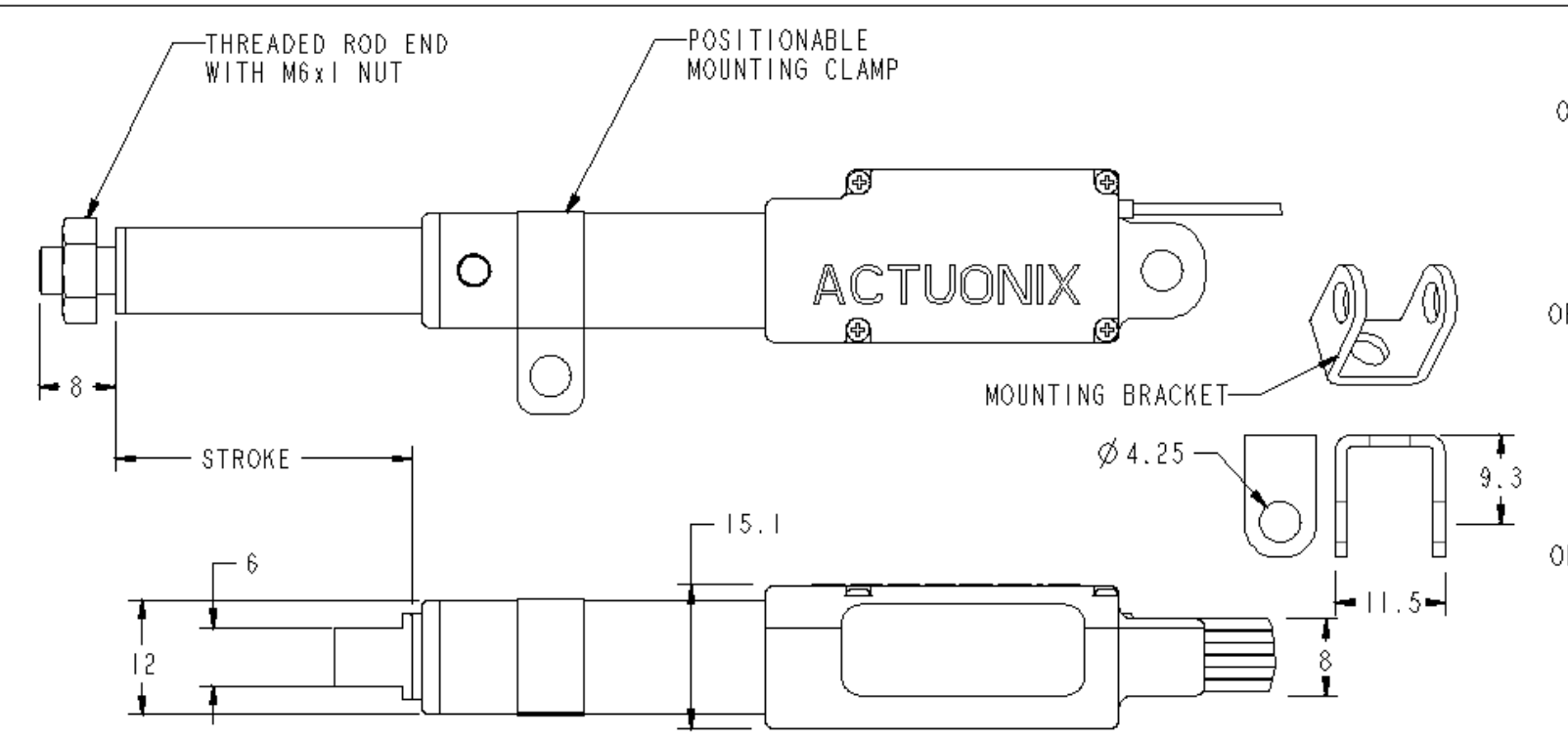

OPTION S

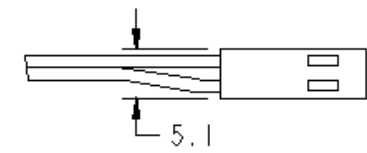

OPTION P

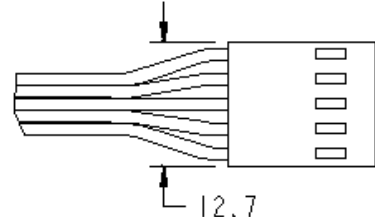

圆 I. ORANGE

OPTION I

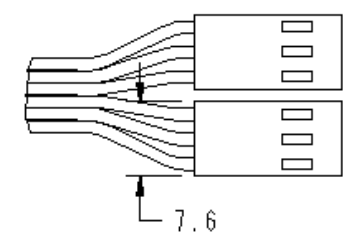

圆 I. GREE

$-7.6$

CLEVIS ROD END-

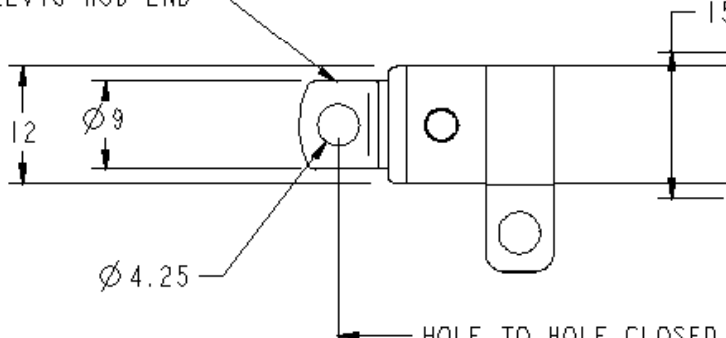

$-15$
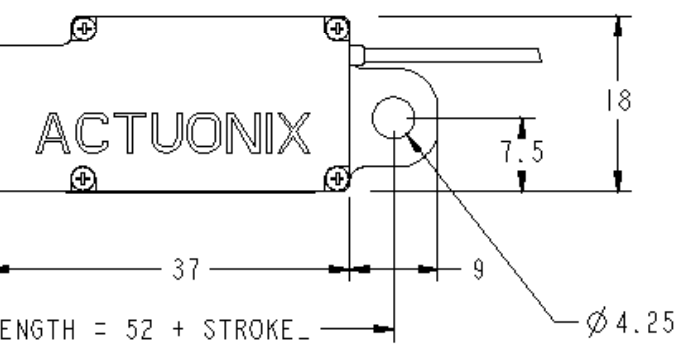

NOTES:

1) WHEN CHANGING ROD ENDS, EXTEND ACTUATOR COMPLETELY

AND HOLD THE ROUND SHAFT WHILE UNSCREWING THE ROD END PLUG

TIGHTEN THE NEW PLUG WITH MAXIMUM TORQUE OF 6 IN-LBS

2) $\varnothing 4.25$ MOUNTING HOLES WILL FIT M4 OR UN \#8 SIZE SCREWS

3) TWO MOUNTING CLAMPS, TWO MOUNTING BRACKETS, ONE EXTRA THREADED ROD END

WITH NUT AND \#8-32 HARDWARE, IS I NCLUDED WITH EACH ACTUATOR.

4) STANDARD LEAD WIRE LENGTH IS $300 \mathrm{~mm}$. 26AWG STRANDED RIBBON WIRE. CONNECTORS ARE 2.54mIn PITCH FEMALE HEADER CONNECTORS
OPTION R

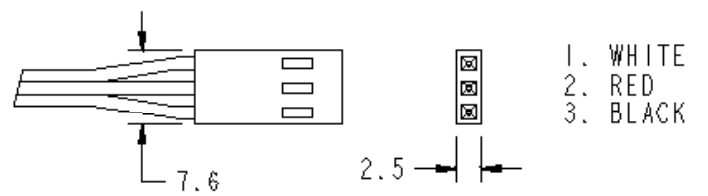

\section{AGTUONIX}

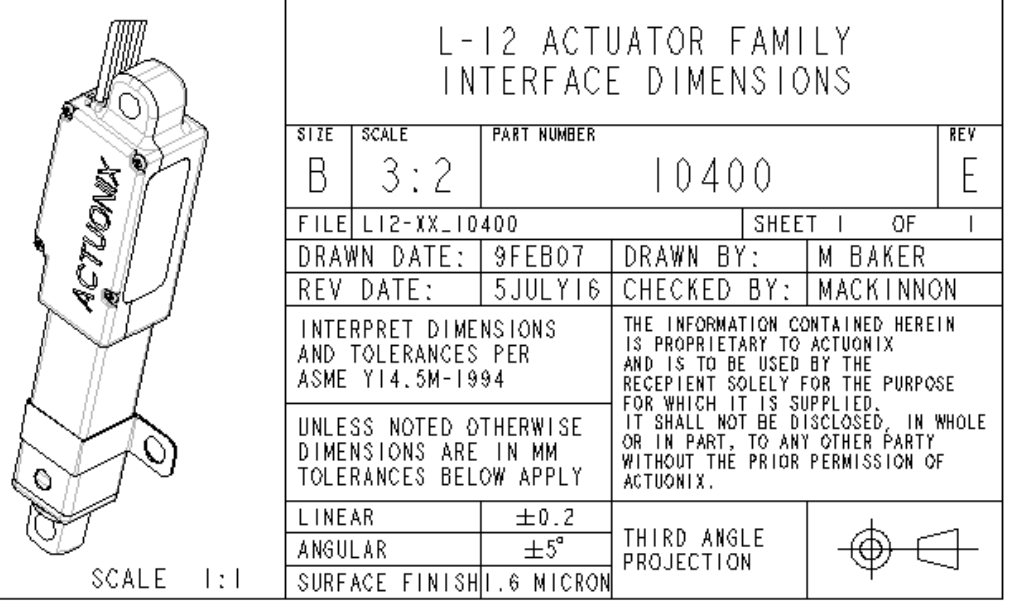

All information provided on this datasheet is subject to change. Purchase or use of Actuonix actuators is subject to acceptance of our term and conditions as posted here: http://www.actuonix.com/terms.asp

Copyright 2016 @ Actuonix Motion Devices Inc. 


\section{LAC • Actuonix Linear Actuator Control Board}

The Linear Actuator Control Board is a stand-alone closed-loop control board specifically designed for Actuonix actuators. The LAC greatly simplifies designs by saving the development time, cost, and processor overhead associated with direct motor control. As little as 1 digital or analog output is required for position control. Supported input signals include USB, Voltage, Current, RC Servo, and PWM. Actuonix motor control IC uses a software based algorithm to optimize position and speed control. This makes the LAC compatible with a wide range of actuators, using only the default settings. Actuonix's Advanced Configuration Program allows full customization of actuator response. A stall detection feature provides a gr eat increase in actuator life for applications that may briefly exceed the rated force.

The LAC can be operated as both an interface board, or as a stand alone controller with the addition of an external potentiometer and power supply.

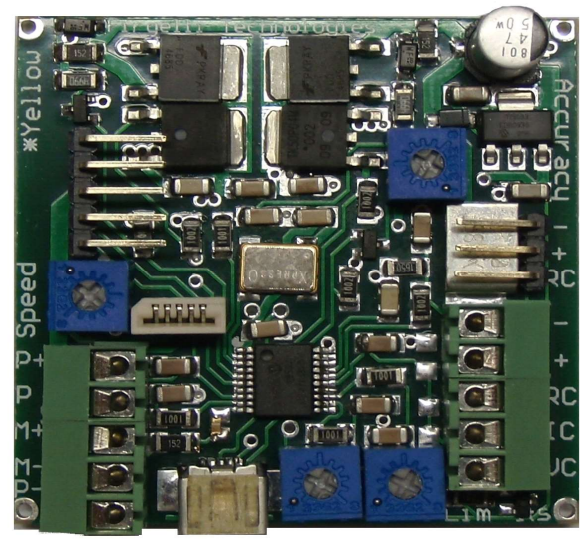

(Accessory kit and housing sold separately)

\section{Specifications}

Control input modes

Controller

Compatible actuators

Dimensions

Power

Operating environment
Digital: USB, RC Servo, $1 \mathrm{kHz}$ PWM

Analog: 0-3.3 V, 4-20 mA

\section{0-bit Dual Sample Rate Quasi PD}

PQ12 \& L12 -P Actuators with position feedback, 6 or 12 volts

L16, P16 \& T16 -P Actuators with position feedback, 6 or 12 volts

Larger Actuators with position feedback, 12 volts, 24 volts

$50 \mathrm{~mm} \times 50 \mathrm{~mm}$ (excluding battery holder)

\section{6-24 VDC, 4 Amps peak current at $10 \%$ duty cycle}

Note: Supply voltage must match actuator's rated voltage

-10 to $+70^{\circ} \mathrm{C}$ at $10-80 \%$ relative humidity

\section{Operation}

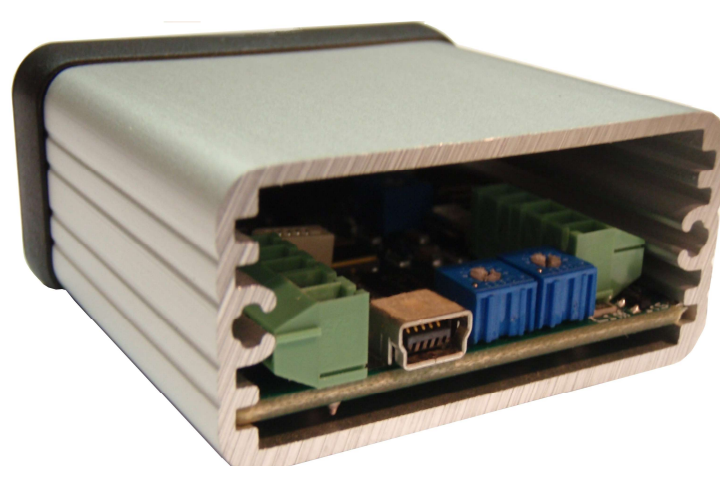

When the LAC is powered up, it will repeatedly scan for an input signal that is valid under any of the five supported interface modes (see reverse for External Connections Detail illustration). When a valid signal is first detected, the actuator will self-configure to the corresponding interface mode, and all other interface modes and input leads are disabled until the actuator is next powered on. The sensitivity or accuracy of the actuator control algorithm can be set by adjusting the "Accuracy" trim potentiometer. Turning clockwise will allow the actuator to move in smaller increments and be more accurate. However, due to the differences in actuator types this may cause jittery or unstable behaviour. If this occurs, consider using the USB configuration program to more finely tune the controller for your application. Each time a control potentiometer is adjusted, power must be cycled to the LAC board prior to the new settings taking effect. Adjusting the "Speed" potentiometer will set the maximum actuator speed. The two "Limits" potentiometers allow user settable digital limit switches. These set the minimum and maximum acceptable positions. Control inputs that exceed these limits will cause the actuator to position to the limit.

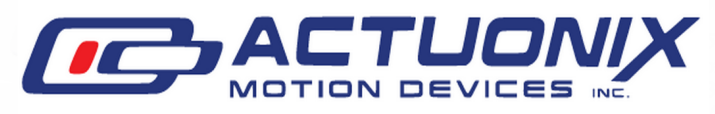

Actuonix Motion Devcies Inc. 1 (206) 347-9684 Phone Unit 201-1753 Sean Heights 1 (888) 225-9198 Toll Free Saanichton, BC Canada 1 (206) 347-9684 Fax V8M 0B3 sales@actuonix.com www.actuonix.com 


\section{External Connections Detail}

\begin{tabular}{|c|c|c|}
\hline X1 PQ12 actuator connector & X6 & Control interface \\
\hline 5 pin, $1 \mathrm{~mm}$ Pitch FPC connector & Pin & Function \\
\hline X2 L12-P/L16-P/P16-P/T16-P & 1 & Ground \\
\hline actuator connector & 2 & 6-24 VDC Power \\
\hline Pin Function & 3 & RC / Hobby Servo input signal \\
\hline 1 Potentiometer Reference Negative (yellow) & 4 & Current input signal $(4-20 \mathrm{~mA})$ \\
\hline 2 Motor Terminal (black) & 5 & Voltage input signal (0-3.3 V) or $1 \mathrm{kHz}$ PWM \\
\hline 3 Motor Terminal (red) & & \\
\hline 4 Potentiometer Feedback (wiper) (purple) & & \\
\hline Potentiometer Reference Positive (orange) & & \\
\hline
\end{tabular}

\author{
P1 Speed Control \\ Sets maximum actuator speed \\ CW Faster \\ CCW Slower \\ P2 Limit Controls \\ Left Potentiometer controls Retract Limit \\ CW Maximum Stroke \\ Right Potentiometer controls Extend Limit \\ CW Maximum Stroke \\ P3 Sensitivity adjustment \\ CW Smaller dead-band \\ CCW Larger dead-band
}

\author{
X3 Radio control receiver connector \\ Pin Function \\ 1 Ground (black) \\ 2 Power (red) \\ 3 Control (white)
}

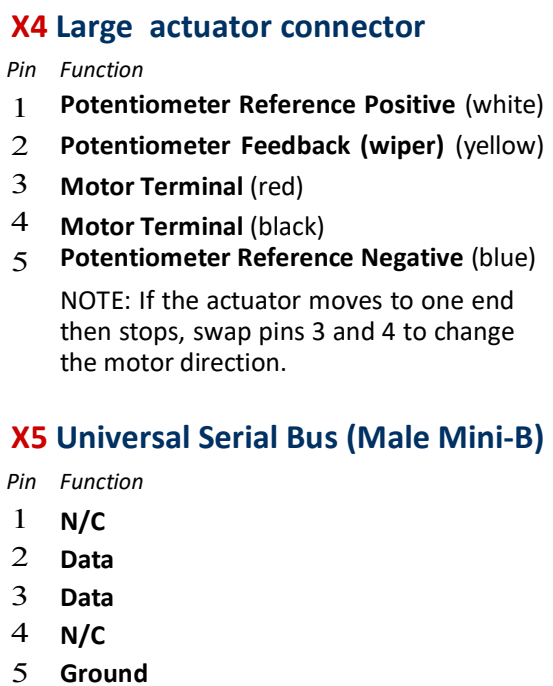

\section{Control Modes}

0-3.3 V Interface Mode: This mode allows an actuator to be controlled with just a battery, and a potentiometer to signal the desired position to the actuator - a simple interface for prototypes or home automation projects. The desired actuator position (setpoint) is input to the CIB on connector X 6 pin 5 as a voltage between ground and $3.3 \mathrm{~V}$. The set-point voltage must be held on pin 5 to reach and maintain the desired actuator stroke position. The wiper pin of an external potentiometer connects to X6 pin 5. Pins 1 and 5 of $X 4$ can be used as the 3.3V Reference. The other two potentiometer pins connect to these. When a Potentiometer is not used, ensure the control signal ground is connected to LAC ground.

RC Servo Interface Mode: This is a standard hobby-type remotecontrol digital servo interface, compatible with servos and receivers from manufacturers like Futaba ${ }^{\mathrm{TM}}$ and $\mathrm{Hi}-\mathrm{Tec}^{\mathrm{TM}}$. The desired actuator position is input to the LAC on connector $X 6$ pin 3 as a positive 5 Volt pulse-width signal. A $1 \mathrm{~ms}$ pulse commands the controller to fully retract the actuator, and a 2 ms pulse signals full extension. Connector X 3 can also be used for the RC control signal, and uses the standard 3 pin 0.1 " spacing typical on most hobby servo receivers. Do not connect power to both X6 and X3 at the same time (If the supply voltages differ, large currents will flow).

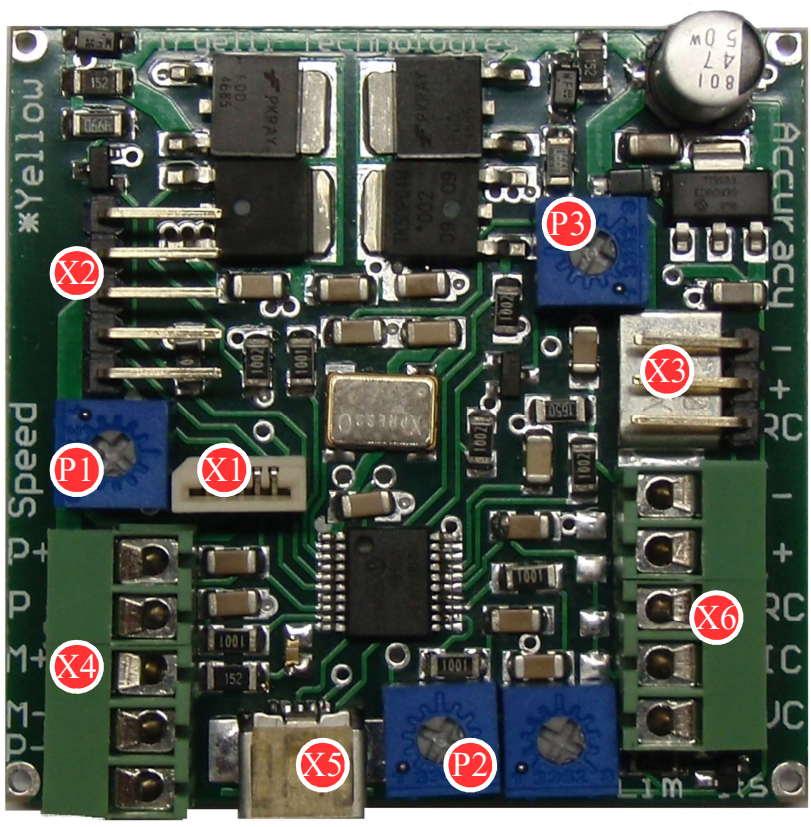

Connector Pins numbered from Top to Bottom or Left to Right

4-20 mA Interface Mode: This mode is compatible with PLC devices typically used in industrial control applications. The desired actuator position (set-point) is input to the LAC on connector X6 pin 4 as a current between $4 \mathrm{~mA}$ and $20 \mathrm{~mA}$. The set-point current must be held on pin 4

to reach and maintain the desired actuator stroke position.

PWM Mode: This mode allows control of the actuator using a single digital output pin from an external micro controller. The desired actuator position is encoded as the duty cycle of a $3.3 \mathrm{Volt}, 1 \mathrm{kHz}$ square wave on LAC connector X6 pin 5, where the percent duty cycle sets the actuator position to the same percent of full stroke extension. $100 \%$ duty cycle represents full extension, and $0 \%$ duty cycle represents full retraction. This input is $5 \mathrm{~V}$ tolerant, however the \% duty cycle range will differ.

USB Mode: This mode allows control of the actuator using a Computer. In addition advanced settings allow fine control over the controller response. Default settings can be reverted to, using the reset command. When custom settings are turned on, P1, P2, and P3 are ignored. These settings will be saved even when power is cycled. This allows custom configuration for all inputs even when USB is not connected. Details of the DLL are given in a separate document so that custom programs can be created by the customer. An example Labview program is available for download. The Dynamic Link Library(DLL) allows Programming in many windows languages including Labview. 


\section{4-Bit Analog-to-Digital Converter (ADC) for Weigh Scales}

\section{DESCRIPTION}

Based on Avia Semiconductor's patented technology, HX711 is a precision 24-bit analogto-digital converter (ADC) designed for weigh scales and industrial control applications to interface directly with a bridge sensor.

The input multiplexer selects either Channel A or B differential input to the low-noise programmable gain amplifier (PGA). Channel A can be programmed with a gain of 128 or 64 , corresponding to a full-scale differential input voltage of $\pm 20 \mathrm{mV}$ or $\pm 40 \mathrm{mV}$ respectively, when a $5 \mathrm{~V}$ supply is connected to AVDD analog power supply pin. Channel B has a fixed gain of 32. Onchip power supply regulator eliminates the need for an external supply regulator to provide analog power for the ADC and the sensor. Clock input is flexible. It can be from an external clock source, a crystal, or the on-chip oscillator that does not require any external component. On-chip poweron-reset circuitry simplifies digital interface initialization.

There is no programming needed for the internal registers. All controls to the HX711 are through the pins.

\section{FEATURES}

- Two selectable differential input channels

- On-chip active low noise PGA with selectable gain of 32, 64 and 128

- On-chip power supply regulator for load-cell and ADC analog power supply

- On-chip oscillator requiring no external component with optional external crystal

- On-chip power-on-reset

- Simple digital control and serial interface: pin-driven controls, no programming needed

- Selectable 10SPS or 80SPS output data rate

- Simultaneous 50 and $60 \mathrm{~Hz}$ supply rejection

- Current consumption including on-chip analog power supply regulator:

normal operation $<1.5 \mathrm{~mA}$, power down $<1 \mathrm{uA}$

- Operation supply voltage range: $2.6 \sim 5.5 \mathrm{~V}$

- Operation temperature range: $-\mathbf{4 0} \sim+85^{\circ} \mathrm{C}$

- 16 pin SOP-16 package

\section{APPLICATIONS}

- Weigh Scales

- Industrial Process Control

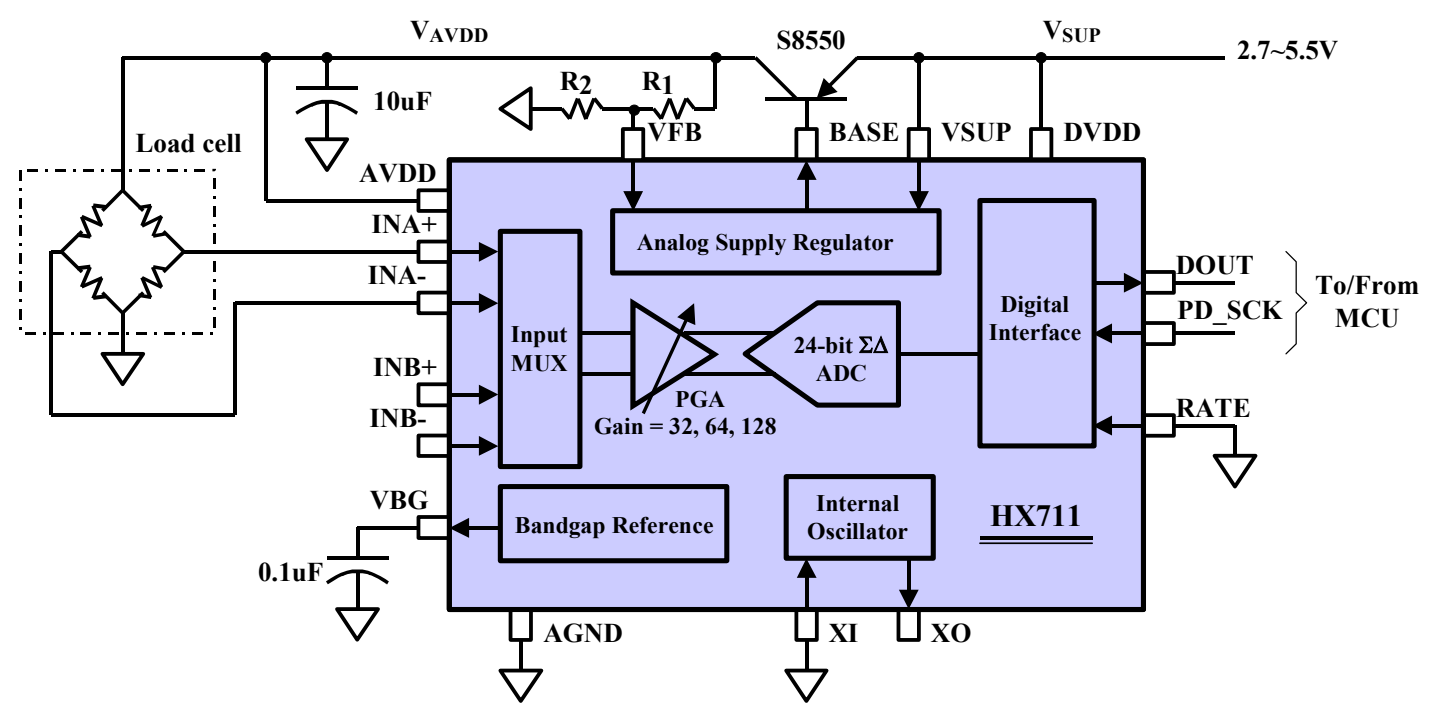

Fig. 1 Typical weigh scale application block diagram 


\section{Pin Description}

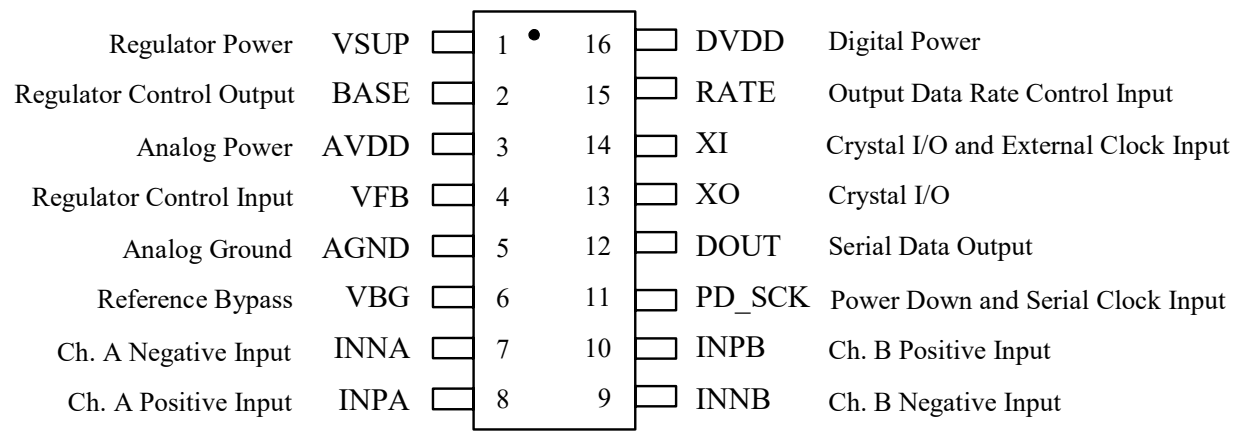

SOP-16L Package

\begin{tabular}{|c|c|l|l|}
\hline Pin \# & Name & \multicolumn{1}{|c|}{ Function } & \multicolumn{1}{|c|}{ Description } \\
\hline \hline 1 & VSUP & Power & Regulator supply: 2.7 5.5V \\
\hline 2 & BASE & Analog Output & Regulator control output (NC when not used ) \\
\hline 3 & AVDD & Power & Analog supply: 2.6 5.5V \\
\hline 4 & VFB & Analog Input & Regulator control input (connect to AGND when not used ) \\
\hline 5 & AGND & Ground & Analog Ground \\
\hline 6 & VBG & Analog Output & Reference bypass output \\
\hline 7 & INA- & Analog Input & Channel A negative input \\
\hline 8 & INA+ & Analog Input & Channel A positive input \\
\hline 9 & INB- & Analog Input & Channel B negative input \\
\hline 10 & INB+ & Analog Input & Channel B positive input \\
\hline 11 & PD_SCK & Digital Input & Power down control (high active) and serial clock input \\
\hline 12 & DOUT & Digital Output & Serial data output \\
\hline 13 & XO & Digital I/O & Crystal I/O (NC when not used ) \\
\hline 14 & XI & Digital Input & Crystal I/O or external clock input, $0:$ use on-chip oscillator \\
\hline 15 & RATE & Digital Input & Output data rate control, $0: 10 \mathrm{~Hz} ; 1: 80 \mathrm{~Hz}$ \\
\hline 16 & DVDD & Power & Digital supply: $2.6 \sim 5.5 \mathrm{~V}$ \\
\hline
\end{tabular}

Table 1 Pin Description 


\section{KEY ELECTRICAL CHARACTERISTICS}

\begin{tabular}{|c|c|c|c|c|c|}
\hline Parameter & Notes & MIN & TYP & MAX & UNIT \\
\hline $\begin{array}{l}\text { Full scale differential } \\
\text { input range }\end{array}$ & V(inp)-V(inn) & \multicolumn{3}{|c|}{ $\pm 0.5(\mathrm{AVDD} / \mathrm{GAIN})$} & V \\
\hline Common mode input & & AGND+1.2 & & AVDD-1.3 & V \\
\hline \multirow{4}{*}{ Output data rate } & Internal Oscillator, RATE $=0$ & & 10 & & \multirow[t]{4}{*}{$\mathrm{Hz}$} \\
\hline & $\begin{array}{l}\text { Internal Oscillator, RATE = } \\
\text { DVDD }\end{array}$ & & 80 & & \\
\hline & $\begin{array}{l}\text { Crystal or external clock, } \\
\text { RATE }=0\end{array}$ & \multicolumn{3}{|c|}{$\mathrm{f}_{\mathrm{clk}} / 1,105,920$} & \\
\hline & $\begin{array}{l}\text { Crystal or external clock, } \\
\text { RATE = DVDD }\end{array}$ & \multicolumn{3}{|c|}{$\mathrm{f}_{\mathrm{clk}} / 138,240$} & \\
\hline Output data coding & 2's complement & 800000 & & 7FFFFF & HEX \\
\hline \multirow{2}{*}{ Output settling time ${ }^{(1)}$} & RATE $=0$ & & 400 & & \multirow[t]{2}{*}{$\mathrm{ms}$} \\
\hline & RATE = DVDD & & 50 & & \\
\hline \multirow{2}{*}{ Input offset drift } & Gain $=128$ & & 0.2 & & \multirow[t]{2}{*}{$\mathrm{mV}$} \\
\hline & Gain $=64$ & & 0.4 & & \\
\hline \multirow{2}{*}{ Input noise } & Gain $=128, \quad$ RATE $=0$ & & 50 & & \multirow[t]{2}{*}{$\mathrm{nV}(\mathrm{rms})$} \\
\hline & Gain $=128$, RATE $=$ DVDD & & 90 & & \\
\hline \multirow{2}{*}{ Temperature drift } & Input offset $($ Gain $=128)$ & & \pm 6 & & $\mathrm{nV} /{ }^{\circ} \mathrm{C}$ \\
\hline & Gain $($ Gain $=128)$ & & \pm 5 & & $\mathrm{ppm} /{ }^{\circ} \mathrm{C}$ \\
\hline $\begin{array}{l}\text { Input common mode } \\
\text { rejection }\end{array}$ & Gain $=128, \quad$ RATE $=0$ & & 100 & & $\mathrm{~dB}$ \\
\hline Power supply rejection & Gain $=128, \quad$ RATE $=0$ & & 100 & & $\mathrm{~dB}$ \\
\hline $\begin{array}{l}\text { Reference bypass } \\
\left(\mathrm{V}_{\mathrm{BG}}\right)\end{array}$ & & & 1.25 & & V \\
\hline $\begin{array}{l}\text { Crystal or external clock } \\
\text { frequency }\end{array}$ & & 1 & 11.0592 & 20 & $\mathrm{MHz}$ \\
\hline \multirow{2}{*}{ Power supply voltage } & DVDD & 2.6 & & 5.5 & \multirow[t]{2}{*}{ V } \\
\hline & AVDD, VSUP & 2.6 & & 5.5 & \\
\hline \multirow[t]{2}{*}{$\begin{array}{l}\text { Analog supply current } \\
\text { (including regulator) }\end{array}$} & Normal & & 1400 & & \multirow[t]{2}{*}{$\mu \mathrm{A}$} \\
\hline & Power down & & 0.3 & & \\
\hline \multirow{2}{*}{ Digital supply current } & Normal & & 100 & & \multirow[t]{2}{*}{$\mu \mathrm{A}$} \\
\hline & Power down & & 0.2 & & \\
\hline
\end{tabular}

(1) Settling time refers to the time from power up, reset, input channel change and gain change to valid stable output data.

Table 2 Key Electrical Characteristics 


\section{Analog Inputs}

Channel A differential input is designed to interface directly with a bridge sensor's differential output. It can be programmed with a gain of 128 or 64 . The large gains are needed to accommodate the small output signal from the sensor. When $5 \mathrm{~V}$ supply is used at the AVDD pin, these gains correspond to a full-scale differential input voltage of $\pm 20 \mathrm{mV}$ or $\pm 40 \mathrm{mV}$ respectively.

Channel B differential input has a fixed gain of 32. The full-scale input voltage range is $\pm 80 \mathrm{mV}$, when $5 \mathrm{~V}$ supply is used at the AVDD pin.

\section{Power Supply Options}

Digital power supply (DVDD) should be the same power supply as the MCU power supply.

When using internal analog supply regulator, the dropout voltage of the regulator depends on the external transistor used. The output voltage is equal to $\mathrm{V}_{\mathrm{AVDD}}=\mathrm{V}_{\mathrm{BG}} *(\mathrm{R} 1+\mathrm{R} 2) / \mathrm{R} 1$ (Fig. 1). This voltage should be designed with a minimum of $100 \mathrm{mV}$ below VSUP voltage.

If the on-chip analog supply regulator is not used, the VSUP pin should be connected to either AVDD or DVDD, depending on which voltage is higher. Pin VFB should be connected to Ground and pin BASE becomes NC. The external 0.1uF bypass capacitor shown on Fig. 1 at the VBG output pin is then not needed.

\section{Clock Source Options}

By connecting pin XI to Ground, the on-chip oscillator is activated. The nominal output data rate when using the internal oscillator is 10 $(\mathrm{RATE}=0)$ or $80 \mathrm{SPS}(\mathrm{RATE}=1)$.

If accurate output data rate is needed, crystal or external reference clock can be used. A crystal can be directly connected across XI and XO pins. An external clock can be connected to XI pin, through a $20 \mathrm{pF}$ ac coupled capacitor. This external clock is not required to be a square wave. It can come directly from the crystal output pin of the MCU chip, with amplitude as low as $150 \mathrm{mV}$.

When using a crystal or an external clock, the internal oscillator is automatically powered down.

\section{Output Data Rate and Format}

When using the on-chip oscillator, output data rate is typically 10 (RATE $=0$ ) or $80 \mathrm{SPS}$ (RATE=1).

When using external clock or crystal, output data rate is directly proportional to the clock or crystal frequency. Using $11.0592 \mathrm{MHz}$ clock or crystal results in an accurate $10(\mathrm{RTE}=0)$ or 80SPS (RATE $=1$ ) output data rate.

The output 24 bits of data is in 2's complement format. When input differential signal goes out of the 24 bit range, the output data will be saturated at $800000 \mathrm{~h}(\mathrm{MIN})$ or 7 FFFFFh (MAX), until the input signal comes back to the input range.

\section{Serial Interface}

Pin PD_SCK and DOUT are used for data retrieval, input selection, gain selection and power down controls.

When output data is not ready for retrieval, digital output pin DOUT is high. Serial clock input PD_SCK should be low. When DOUT goes to low, it indicates data is ready for retrieval. By applying 25 27 positive clock pulses at the PD_SCK pin, data is shifted out from the DOUT output pin. Each PD_SCK pulse shifts out one bit, starting with the MSB bit first, until all 24 bits are shifted out. The $25^{\text {th }}$ pulse at PD_SCK input will pull DOUT pin back to high (Fig.2).

Input and gain selection is controlled by the number of the input PD_SCK pulses (Table 3). PD_SCK clock pulses should not be less than 25 or more than 27 within one conversion period, to avoid causing serial communication error.

\begin{tabular}{|c|c|c|}
\hline PD_SCK Pulses & $\begin{array}{c}\text { Input } \\
\text { channel }\end{array}$ & Gain \\
\hline \hline 25 & A & 128 \\
\hline 26 & B & 32 \\
\hline 27 & A & 64 \\
\hline
\end{tabular}

Table 3 Input Channel and Gain Selection 


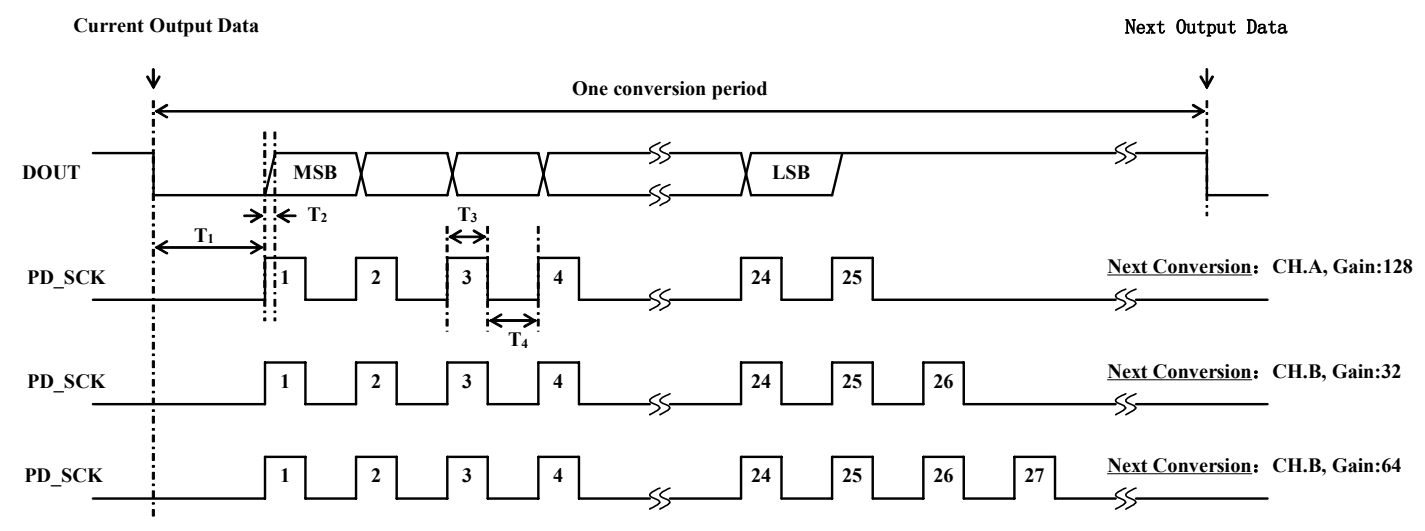

Fig.2 Data output, input and gain selection timing and control

\begin{tabular}{|c|l|c|c|c|c|}
\hline Symbol & \multicolumn{1}{|c|}{ Note } & MIN & TYP & MAX & Unit \\
\hline \hline $\mathrm{T}_{1}$ & DOUT falling edge to PD_SCK rising edge & 0.1 & & & $\mu \mathrm{s}$ \\
\hline $\mathrm{T}_{2}$ & PD_SCK rising edge to DOUT data ready & & & 0.1 & $\mu \mathrm{s}$ \\
\hline $\mathrm{T}_{3}$ & PD_SCK high time & 0.2 & 1 & 50 & $\mu \mathrm{s}$ \\
\hline $\mathrm{T}_{4}$ & PD_SCK low time & 0.2 & 1 & & $\mu \mathrm{s}$ \\
\hline
\end{tabular}

\section{Reset and Power-Down}

When chip is powered up, on-chip power on rest circuitry will reset the chip.

Pin PD_SCK input is used to power down the HX711. When PD_SCK Input is low, chip is in normal working mode.

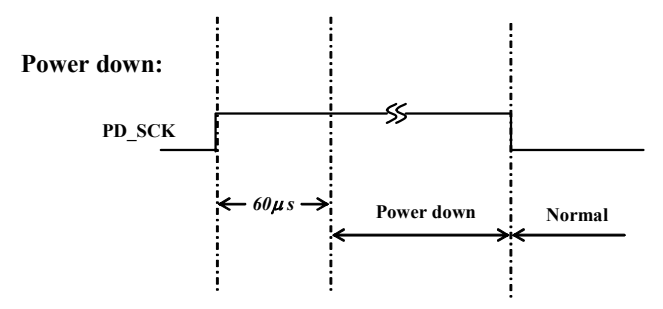

Fig.3 Power down control

When PD_SCK pin changes from low to high

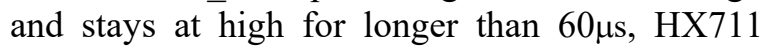
enters power down mode (Fig.3). When internal regulator is used for HX711 and the external transducer, both HX711 and the transducer will be powered down. When PD SCK returns to low, chip will reset and enter normal operation mode.

After a reset or power-down event, input selection is default to Channel A with a gain of 128.

\section{Application Example}

Fig. 1 is a typical weigh scale application using $\mathrm{HX} 711$. It uses on-chip oscillator $(\mathrm{XI}=0), 10 \mathrm{~Hz}$ output data rate $(\mathrm{RATE}=0)$. A Single power supply $(2.7 \sim 5.5 \mathrm{~V})$ comes directly from $\mathrm{MCU}$ power supply. Channel B can be used for battery level detection. The related circuitry is not shown on Fig. 1. 
Reference PCB Board (Single Layer)

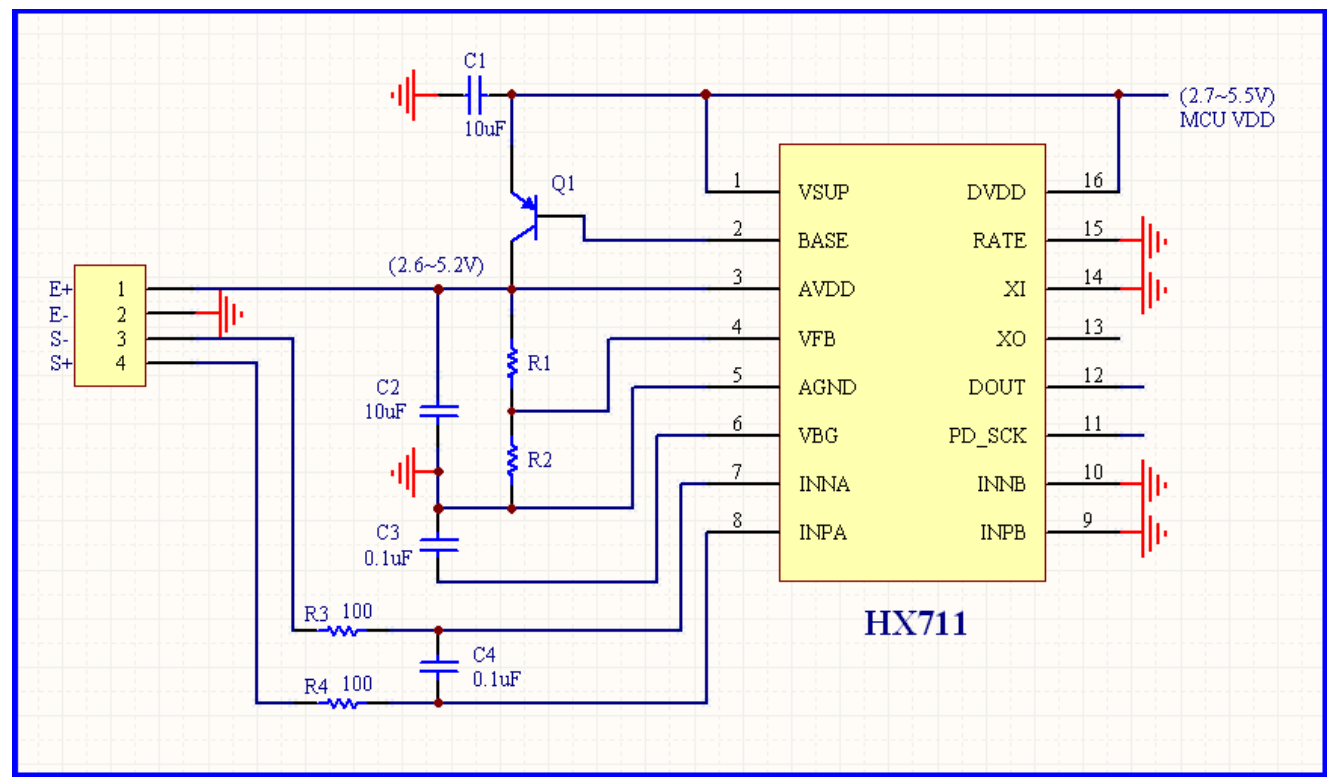

Fig.4 Reference PCB board schematic

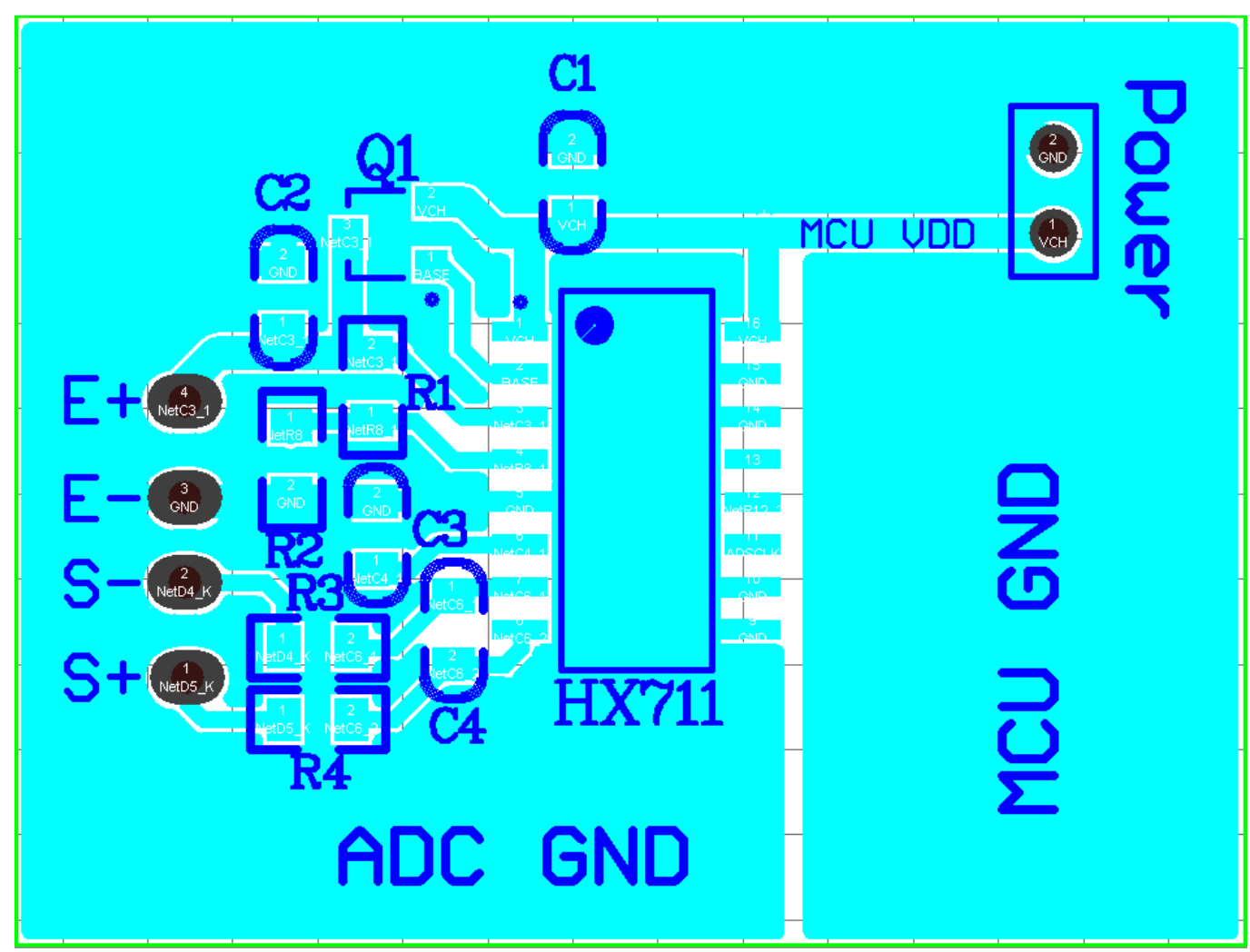

Fig.5 Reference PCB board layout 


\section{Reference Driver (Assembly)}

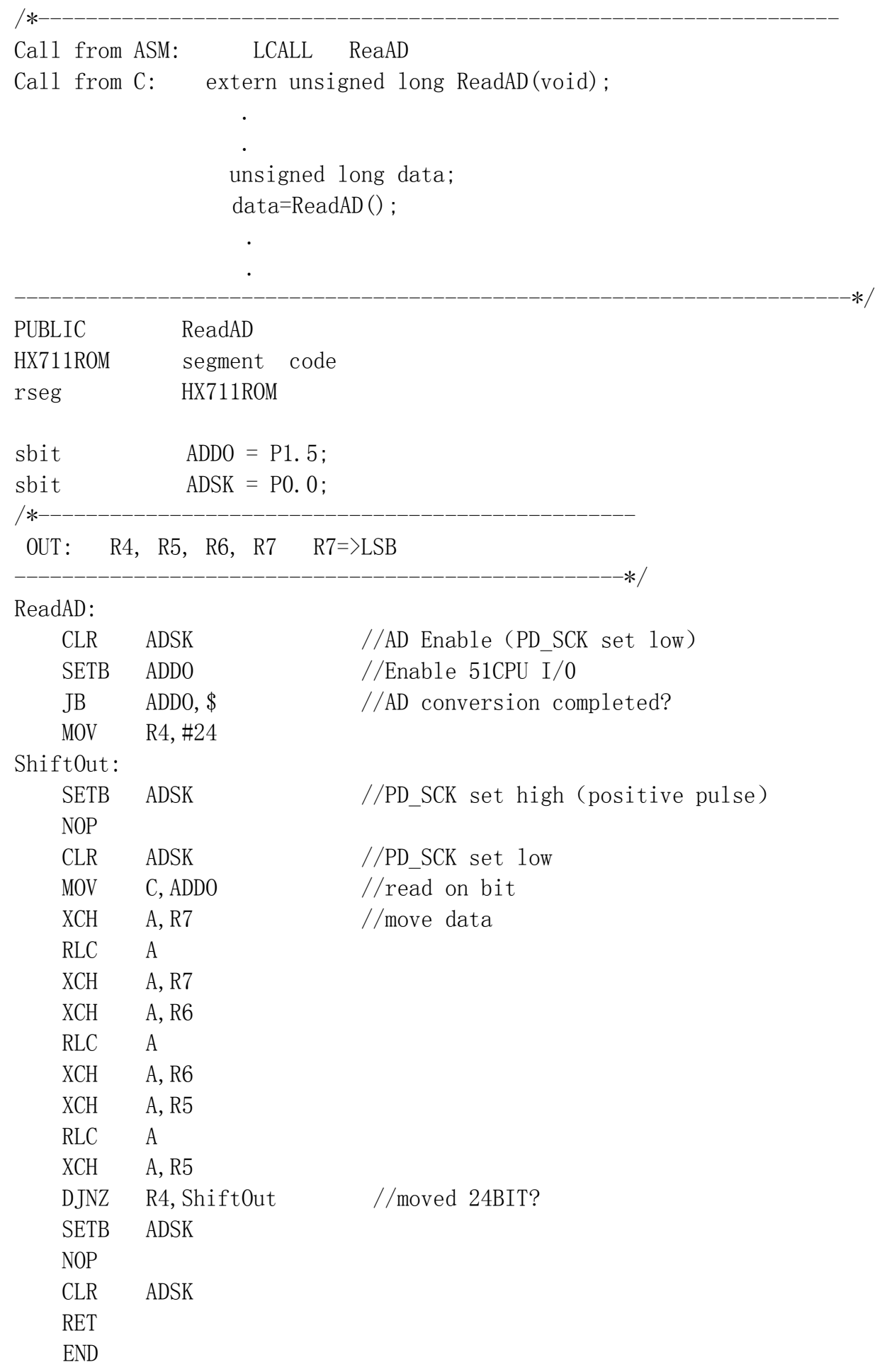




\section{Reference Driver（C)}

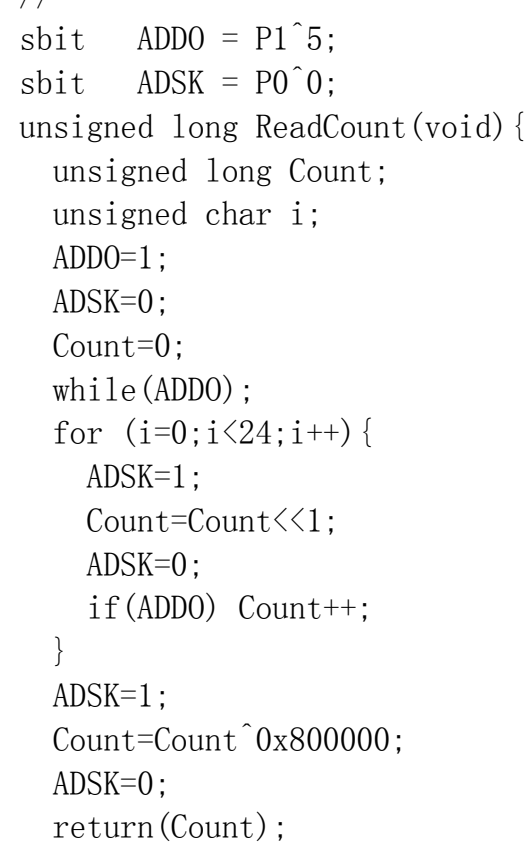




\section{Package Dimensions}
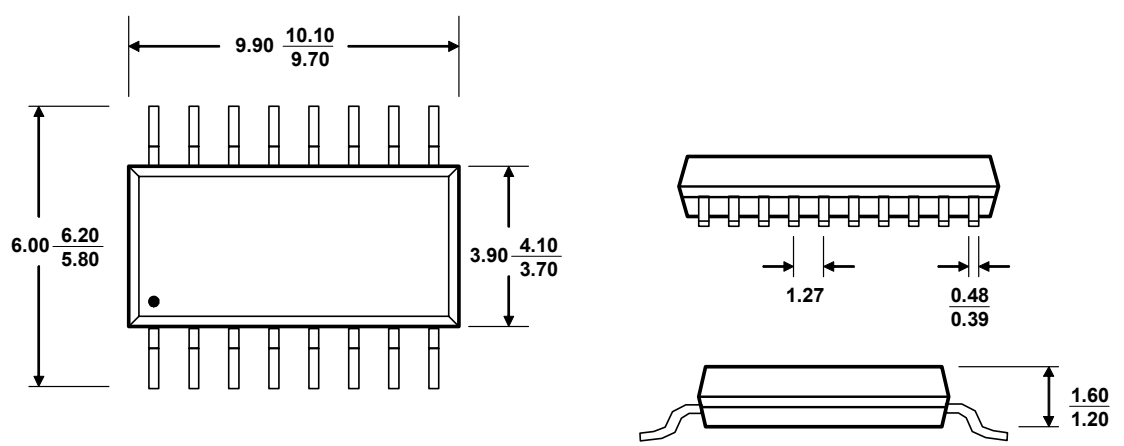

Typ $\frac{\text { MAX }}{\text { MIN }} \quad$ Unit: mm

SOP-16L Package 